\title{
Pares de Higgs, grassmanniana infinita y sistemas integrables
}

\author{
Daniel Hernández Serrano
}

Tesis Doctoral dirigida por

José María Muñoz Porras

Francisco José Plaza Martín

Departamento de Matemáticas

Universidad de Salamanca 



\title{
Pares de Higgs, grassmanniana infinita y sistemas integrables
}

\author{
Tesis presentada por \\ Daniel Hernández Serrano \\ para optar al título de \\ Doctor por la Universidad de Salamanca
}

Daniel Hernández Serrano, Doctorando

José María Muñoz Porras, Director

Francisco José Plaza Martín, Codirector

Departamento de Matemáticas

Universidad de Salamanca 



\section{Agradecimientos}

En primer lugar, me gustaría dedicar este trabajo a mis padres, Daniel y Laína, a mis hermanos, Juan y Gloria, y a mi tío Manolo, a quienes quiero y admiro profundamente, y de los que constantemente aprendo y disfruto. He tenido la suerte de contar con dos matemáticos dentro de casa, mis padres, que no sólo han conseguido enseñarme pacientemente durante las etapas que llevo recorridas (digamos que no les quedaba otra), sino que también han conseguido que disfrute con ello. Todo un lujo asiático y un divertido defecto genético. En segundo lugar se lo dedico a Rocío, que cariñosamente se ha encargado de cuidarme y aguantarme durante estos años de trabajo, y que ha hecho de ello algo ya inolvidable.

Desde luego, este trabajo no hubiera sido posible sin la ayuda, orientación y apoyo constante de mis directores de Tesis, Porras y Paco, a quienes agradezco enormemente la confianza que han depositado en mí, y la fantástica formación que amable y desinteresadamente me están dando. A ellos les dedico un sincero testimonio de admiración. Me gustaría también agradecer los valiosos comentarios y las acertadas correcciones con los que Esteban y mi padre me han obsequiado.

Aparte de los compañeros ya mencionados, a lo largo de estos años también he disfrutado particularmente de otros como Carlos, Cristina, José Ángel, Fernando, los Sancho,... todos ellos profesores de este agradable Departamento, de los que voy aprendiendo la profesión, y con los que he pasado grandes ratos tomando café y charlando.

De todos mis amigos de carrera (algunos también colegas de profesión), como César, Galante, Marga, Curtis, Cristian, Oscar, Marta, Cristina, ... me gustaría destacar muy especialmente a Darío, con quien he compartido y comparto muy buenos años de verdadera amistad personal y profesional. Quiero también dar las gracias a mis amigos de toda la vida, Miguel, Gorka, Alfonso, Ángel, Gaspi, Ernes, Soto, Ámbar, Javi Amado, Paz, Kuka, Pepe, Beni, Mc Berrocal, Toño, Michel, ... que siempre han estado ahí, escuchándome (no sólo con la dichosa guitarra), aconsejándome y haciéndome reír.

Quiero así mismo hacer constar en estas palabras, los nombres de la gente que he ido conociendo en congresos y seminarios a lo largo de todo este tiempo, con los que he compartido interesantes conversaciones convenientemente aderezadas con cerveza: Luis, Marina, Roberto, Mario, Steve, Álvaro, Emolo, Jarek, Álvaro, Ale, Caravantes, Nacho, Andrea, ...

Por último, quiero dar la gracias al Ministerio de Educación y Cultura, con cuya financiación me ha sido posible realizar este trabajo. 

Estos son mis principios.

Si a usted no le gustan, tengo otros.

Groucho 



\section{Índice general}

\begin{tabular}{ll|}
\hline 0. Introducción. & 1
\end{tabular}

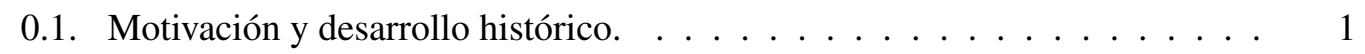

$0.2 . \quad$ Objetivos y resultados. . . . . . . . . . . . . . . . . . . . . . . . 3

$0.3 . \quad$ Estructura del trabajo. . . . . . . . . . . . . . . . . 5

\begin{tabular}{|ll|}
\hline I & Esquemas en grupos y sus álgebras de Lie.
\end{tabular}

\begin{tabular}{lr}
\hline 1. Grupos formales. & 9
\end{tabular}

1.1. Grupo de invertibles. . . . . . . . . . . . . . . . . . . . 9

1.2. Grupos de automorfismos. . . . . . . . . . . . . . . . . . . . . . 10

1.3. Grupo de Heisenberg y grupo de Weyl. . . . . . . . . . . . . . . . . . . 17

1.4. Grupos de automorfismos semilineales. . . . . . . . . . . . . . . . . . . 19

2. Álgebras de Lie de los grupos formales. 25

2.1. Álgebra de Lie de los grupos de automorfismos. . . . . . . . . . . . . . . 25

2.2. Operadores diferenciales como álgebras de Lie. . . . . . . . . . . . . . 27

\begin{tabular}{llr}
\hline II Espacios de Moduli & 33
\end{tabular}

\begin{tabular}{|r|r}
\hline 3. Moduli de curvas y fibrados. & 35
\end{tabular}

3.1. Morfismo de Krichever. . . . . . . . . . . . . . . . . . . . . . 35

3.2. Caracterización de la imagen. . . . . . . . . . . . . . . . . . 40

3.3. Representabilidad. . . . . . . . . . . . . . . . . . . 45

3.4. Caso singular. . . . . . . . . . . . . . . . . . . 47

4. Pares de Higgs y Grassmanniana Infinita. 49

4.1. Preliminares. . . . . . . . . . . . . . . . . . . . 49

4.2. Moduli de pares de Higgs con trivializaciones formales. . . . . . . . . . . 51

4.3. Construcción espectral y la Grassmanniana infinita relativa. . . . . . . . . . 60 
5. Moduli de haces de línea en revestimientos y sistemas integrables. 63

5.1. Moduli de revestimientos de grado $n$. . . . . . . . . . 63

5.2. Moduli de haces de línea sobre revestimientos. . . . . . . . . . . . . . 71

5.3. Sistemas integrables. . . . . . . . . . . . . . . . . . 74

5.4. Fibrados que son imagen directa de haces de línea. . . . . . . . . . . . 76

\begin{tabular}{lll}
\hline III Estudio infinitesimal. & 81
\end{tabular}

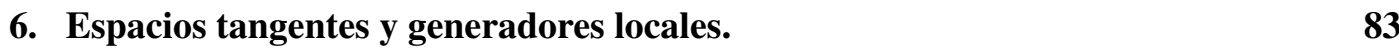

6.1. Moduli de fibrados sobre una curva lisa fija. . . . . . . . . . . . . . . . 87

6.2. Moduli de fibrados y curvas lisas. . . . . . . . . . . . . . . . . . . . 90

6.3. Moduli de revestimientos. . . . . . . . . . . . . . . . . . . . . . . . 97

6.4. Moduli de revestimientos y haces de línea. . . . . . . . . . . . . . . . . . . 104

6.5. Moduli de pares de Higgs. . . . . . . . . . . . . . . . . . . 108

\begin{tabular}{|c|c|}
\hline 7. Ecuaciones del moduli de pares de Higgs. & 111
\end{tabular}

7.1. Geometría formal. . . . . . . . . . . . . . . . . . . . 111

7.2. Ecuaciones del moduli. . . . . . . . . . . . . . . . . . . . . . . . . . 119

\begin{tabular}{|l|l}
\hline IV Apéndices. & 125
\end{tabular}

\begin{tabular}{ll}
\hline A. Grassmanniana Infinita. & 127
\end{tabular}

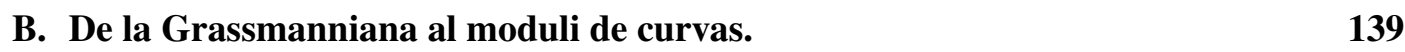

\begin{tabular}{ll}
\hline C. Operadores diferenciales. & 143
\end{tabular}

\begin{tabular}{|rr}
\hline D. Quick overview of the work. & 149
\end{tabular}

D.0. Introduction. . . . . . . . . . . . . . . . . . . . . . . . . . 149

D.1. Group schemes. . . . . . . . . . . . . . . . . . . . . . . . 152

D.2. Lie algebras of the group schemes. . . . . . . . . . . . . . . . . 156

D.3. Moduli of curves and vector bundles. . . . . . . . . . . . . . . . . . 157

D.4. Moduli of Higgs Pairs. . . . . . . . . . . . . . . . . . . . . . . . . . . . . 159

D.5. Moduli of coverings and integrable systems. . . . . . . . . . . . . . . . . 164

D.6. Tangent spaces and local generators. . . . . . . . . . . . . . . . . . 170

D.7. Equations of the moduli of Higgs pairs. . . . . . . . . . . . . . . . . 174

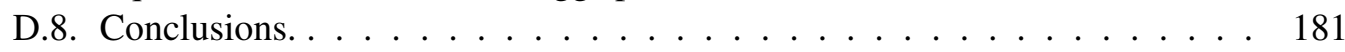

Índice Alfabético $\quad 185$

$\begin{array}{ll}\text { Bibliografía } & 187\end{array}$ 


\section{Capítulo o}

\section{Introducción.}

\subsection{Motivación y desarrollo histórico.}

Durante los años 77-81, Sato encontró una equivalencia entre las soluciones del sistema KP y puntos de una Grassmanniana infinita ([40]) y Krichever observó una estrecha relación entre esta teoría y las funciones theta de Jacobianas de curvas ([22]). En el mismo periodo y con las mismas herramientas, Mumford ([30]) desarrolló completamente un diccionario entre diferentes objetos geométricos (curvas punteadas, haces de línea, fibrados parabólicos, ...) y subanillos conmutativos de cierto anillo no conmutativo de operadores. Todas estas aportaciones contribuyeron a desarrollar un nuevo e interesante punto de vista algebro-geométrico del estudio de la conmutatividad de operadores diferenciales llevada a cabo por Burchnall y Chaudy ([11]) en los años 20.

Teniendo en cuenta estas ideas y su relación con el problema de Schottky, en [27] (1984) Mulase caracterizó las Jacobianas como órbitas finito-dimensionales de determinados flujos en la Grassmanniana de Sato, y Shiota dió en [44] una condición necesaria y sufienciente para que la función theta de una variedad abeliana principalmente polarizada fuera la función theta de la Jacobiana de una curva. En 1985 Segal y Wilson formalizaron en [42] el morfismo de Krichever (aplicación entre ciertos objetos geométricos y la Grassmanniana de Sato) e hicieron un especial énfasis en cómo los grupos de lazos (loop groups) podían encajar en estos sistemas infinitos.

En el año 1991, Mulase contribuyó de nuevo a desarrollar estas ideas dando una equivalencia categorial entre fibrados vectoriales sobre curvas y la Grassmanniana de Sato ([28]). En [24], Li y Mulase presentan el sistema KP como ciertos flujos conmutativos en la Grassmanniana de Sato y prueban que toda órbita finito-dimensional de estos flujos es canónicamente isomorfa a la Jacobiana de una curva (y recíprocamente). Además, ellos relacionan las variedades de Prym y el sistema BKP. En la misma línea, Adams y Bergvelt estudiaron el caso de revestimientos finitos de curvas con haces de línea ([1], 1992), demostrando que 
las álgebras de Heisenberg definen flujos en la Grassmanniana de Sato y producen clases de soluciones de jerarquías KP. Dieron además, una detallada correspondencia entre datos geométricos, álgebras de Heisenberg y puntos de la Grassmanniana de Sato.

En [3] (1996), Álvarez Vázquez, Muñoz Porras y Plaza Martín dotan a la Grassmanniana de Sato de una estructura algebraica, la convierten en un esquema. Este hecho, les permitó dar una teoría algebraica de la jerarquía KP sobre un cuerpo de característica arbitraria, construir algebraicamente las funciones Tau y de Baker-Akhiezer (lo que les permite calcular explicitamente ecuaciones de tipo KP) y dotar de una estructura de esquema a determinados espacios de moduli ([33, 37]). Siguiendo las ideas de Segal ([41]), Muñoz Porras y Plaza Martín se encargan de estudiar el grupo automorfismos del cuerpo de desarrollos formales de Laurent, presentan dicho grupo (grupo de Virasoro) como generador local del moduli de curvas y, dado que su teoría es independiente del género, esto les permite formular una Teoría Conforme de Campos no perturbativa ([34]). Así mismo, el grupo $\Gamma$ de invertibles en el cuerpo de desarrollos formales de Laurent (que aparece en [3, 42]) uniformiza la Jacobiana de una curva y permite linearizar ciertos flujos en la Grassmanniana de Sato. Este esquema en grupos $\Gamma$ es la versión algebraica del loop group de $\mathcal{S}^{1}$.

En [31] las jerarquías de soliton aparecen de manera natural al estudiar los esquemas de Hurwitz, están dadas por flujos en la Grassmanniana definidos por álgebras de Heisenberg. Utilizan para ello la aplicación traza como ingrediente clave para caracterizar la imagen del morfismo de Krichever y calcular las ecuaciones diferenciales de los esquemas de Hurwitz, aportando así una aproximación diferente a los problemas tratados en [1, 24]. Estos espacios de Hurwitz son espacios de revestimientos $Y \rightarrow X$ finitos entre curvas (íntegras y lisas), y por tanto, podemos pensar el cuerpo de funciones de $Y$ como una extensión del cuerpo de funciones de $X$, lo que permite dar una visón geométrica de la Teoría de Cuerpos de Clases y encajar ciertos resultados dentro del Programa de Langlands (como una versión no abeliana de la la Teoría de Cuerpos de Clases). En esta dirección, hay resultados enormemente interesantes, entre los que cabe destacar los trabajos de Frenkel y Ben-Zvi ([8, 15, 14, 13]), donde además se pone de manifiesto la utilidad de las herramientas que nos proporciona la Grassmanniana infinita.

Por último, otro factor clave para este trabajo es la teoría de los pares de Higgs. Expliquemos brevemente por qué determinados matemáticos decidieron atacar el sistema integrable que de ellos surge desde el punto de vista de la Grassmanniana infinita. Retrocedamos hasta 1988, en ese año, y motivado por una abelianización (o linearización) del estudio de los fibrados vectoriales (lo que actualmente se conoce como el Programa de Abelianización de Hitchin), Hitchin centra su investigación en la Geometría Simpléctica del espacio cotangente al moduli de fibrados vectoriales sobre una superficie de Riemann compacta, sus puntos le llevaron a introducir un nuevo, y actualmente relevante concepto: la noción de pares de Higgs. Desde el punto de vista de la Geometría Simpléctica, la aplicación que va desde dicho espacio contangente a un espacio afín de secciones globales (ahora llamada morfismo de Hitchin), resultó ser un sistema Hamiltoniano algebraicamente completamente integrable, que esencialmente significa que las fibras del morfismo de Hitchin son Jacobianas de ciertas curvas (las curvas espectrales). Como el propio autor dijo en [21], queda una cuestión 
abierta: cómo realizar, de una manera concreta, estas ecuaciones diferenciales Hamiltonianas. Teniendo en mente esta pregunta, Li y Mulase resuelven parcialmente el problema en [23] utilizando la Grassmanniana de Sato como un espacio de soluciones para la jerarquía KP. Ellos responden a la cuestión de cómo recuperar el sistema de Hitchin a partir del sistema $\mathrm{KP}$ en el caso en que el revestimiento espectral es no ramificado. Paralelamente, y usando técnicas similares (morfismo de Krichever, Grassmanniana de Sato, ...), Donagi y Markman estudian este tópico ([12]) probando la existencia de una cierta compatibilidad para los flujos KP que provienen de datos espectrales asociados a pares de Higgs en una curva fija. Recientemente, Mulase y Hodge han puesto de manifiesto ([29]) la utilidad de estas herramientas tanto en el contexto del Programa de Langlands como en el de la llamada Mirror Symmetry.

\subsection{Objetivos y resultados.}

Los objetivos marcados para este trabajo han sido, en primer lugar profundizar en la pregunta de Hitchin que antes mencionamos, complementando los resultados de [23, 12], y buscar otros sistemas integrables que se comporten de modo análogo; en segundo lugar, calcular esquemas en grupos que generen localmente los espacios de moduli. Este segundo objetivo no sólo tiene interés en sí mismo o en el Programa Geométrico de Langlands, sino que puede enmarcarse también dentro de la teoría de cuerdas bosónicas (como se pone de manifiesto en [34]), resultar beneficioso a la hora de calcular la cohomología de espacios de moduli estandar (esto es, sin trivializaciones formales), como se hace en [4], y también permite pensar los espacios de moduli estandar como stacks cocientes de estos grupos, lo que se puede aprovechar, por ejemplo, para demostrar determinadas "dualidades extrañas" (strange dualities) de la Física.

La táctica general que hemos seguido consiste en hacer uso de la estructura de esquema de la Grassmanniana de Sato, para sumergir en ella (gracias al morfismo de Krichever) espacios de moduli y dotarlos de una estructura de esquema, probando que son espacios de moduli finos. Una de las grandes ventajas del morfismo de Krichever, es que permite codificar complicados espacios de moduli en condiciones algebraicas ciertamente sencillas, que los caracterizan plenamente dentro de Grassmannianas de Sato, y demostrar así mismo la existencia de espacios de moduli finos (como el moduli de fibrados) sin imponer las condiciones de estabilidad de la Teoría Geométrica de Invariantes de Mumford. Además, esto es tan útil como efectivo en las dos aplicaciones que hemos escogido: el cálculo de espacios tangentes y el cálculo de ecuaciones de tipo KP.

Los resultados obtenidos en esta tesis son los siguientes:

1. Dar un morfismo de Krichever para el moduli $\mathcal{U}^{\infty}$ de fibrados vectoriales y curvas con trivializaciones formales (no admitiendo una estructura local de revestimiento cíclico alrededor del punto fijado, complementado así los resultados de Mulase en [28]) y dotar a este moduli de una estructura de esquema. Obtenemos de este modo una caracterización à la Mulase de los puntos de la imagen del morfismo de Krichever sólo en términos del punto de la Grassmanniana infinita definido por el fibrado vectorial. 
2. Calcular el espacio tangente a $\mathcal{U}^{\infty}$ y encontrar un esquema en grupos que lo genere localmente. Este nuevo esquema en grupos es el grupo de automorfismos semilineales de un cuerpo de desarrollos de Laurent, y su álgebra de Lie es un álgebra de operadores diferenciales. Generalizamos así algunos de los resultados de [4] y [32], donde se estudia este problema para haces de línea.

3. Caracterizar la imagen del morfismo de Krichever para el moduli $\mathcal{H i g g s}_{X}^{\infty}$ de pares de Higgs sobre una curva lisa $X$ con trivializaciones formales y representar dicho moduli como un subesquema dentro de una Grassmanniana infinita. La Grassmanniana que necesitamos para definir el morfismo de Krichever es una fibración de Grassmannianas infinitas $\mathcal{G}$ r sobre el espacio afín $\mathbb{A}$ de los polinomios mónicos con coeficientes en $k[[z]]$, que no es sino un análogo formal a la llamada base de Hitchin. Demostramos que $\mathcal{H i g g s}_{X}^{\infty}$ es representable, que el morfismo de Krichever es inyectivo y caracterizamos su imagen. Esto nos permite demostrar a la postre que $\mathcal{H} i g g s_{X}^{\infty}$ es representable por un subesquema cerrado de $\mathcal{U}_{X}^{\infty} \times \mathbb{A}$.

4. Caracterizar la imagen del morfismo de Krichever para el moduli $\mathcal{C}_{o v}{ }^{\infty}$ de revestimientos finitos y punteados de curvas y el moduli $\mathcal{P} i c^{\infty}$ de haces de línea sobre dichos revestimientos (siempre con trivializaciones formales). La diferencia principal con los espacios de moduli estudiados en [32, 31] radica en el hecho de que nosotros no imponemos compatibilidad entre las trivializaciones formales, recuperamos por tanto sus resultados como casos particulares. Usando técnicas similares a las de [32, 31] dotamos a estos espacios de moduli con una estructura de esquema dentro de la Grassmanniana relativa $\mathcal{G}$ r.

5. Encontrar un sistema integrable con propiedades análogas al sistema de Hitchin. Si fijamos la curva base $X$ y nos restringimos al subconjunto $\mathbb{O}$ de $\mathbb{A}$ de las funciones algebraicas, la construcción de $\mathcal{P} i c^{\infty}$ implica la existencia de un esquema $\mathcal{P} i c_{X}^{\infty} \rightarrow \mathbb{O}$ que parametriza los esquemas de Picard de todos los revestimientos finitos algebraicos de $X$, esto es, la existencia de un sistema integrable con propiedades similares a las del sistema de Hitchin. Además, resulta que $\mathcal{P} i c_{X}^{\infty}$ es un subesquema de $\mathcal{U}_{X}^{\infty}$ y se tiene un morfismo epiyectivo $\mathcal{P} i c_{X}^{\infty} \rightarrow \mathcal{U}_{X}$, donde $\mathcal{U}_{X}$ denota el stack de fibrados vectoriales sobre $X$, por lo tanto, encontramos aquí una relación con el programa de Abelianización de Hitchin. Hemos también de destacar que $\mathcal{H} i g g s_{X}^{\infty}$ es un subesquema de $\mathcal{P} i c_{X}^{\infty}$. Esperamos además que estos resultados nos permitan dar una nueva construcción de la curvas espectrales en el sentido de [7].

6. Calcular los espacios tangentes a $\operatorname{Cov}^{\infty}$ y $\mathcal{P} i c^{\infty}$ y encontrar esquemas en grupos que los generen localmente. Estos esquemas en grupos son, en el primer caso, una generalización del grupo de Virasoro estudiado en [34, 32] (es un grupo de automorfismos de un cuerpo de desarrollos de Laurent), y en el segundo, un grupo de automorfismos semilineales cuya álgebra de Lie es un álgebra de operadores diferenciales, que puede obtenerse como una extensión del álgebra de Heisenberg estudiada en [1]. 
7. Calcular jerarquías de ecuaciones diferenciales que caracterizan el moduli de pares de Higgs, $\mathcal{H}_{\text {iggs }}^{\infty}{ }_{X}^{\infty}$. Esta jerarquía es equivalente a las ecuaciones algebraicas de $\mathcal{H} i g g s_{X}^{\infty}$ como subesquema de la Grassmanniana. Los ingredientes clave para dar estas ecuaciones son, por un lado la caracterización de la imagen del morfismo de Krichever del punto 3 y por otro, el hecho de reformular la geometría formal descrita en [3] y [31], lo que nos ha permitido dar las ecuaciones en términos de funciones de BakerAkhiezer. Recuperamos así, y de un modo distinto, el caso estudiado en [23] (revestimiento espectral no ramificado). Además, para el subesquema de $\mathcal{H i g g s}_{X}^{\infty}$ en el que el revestimiento espectral está totalmente ramificado en el punto que fijamos, damos las ecuaciones sólo en términos de los coeficientes del polinomio característico del campo de Higgs.

\subsection{Estructura del trabajo.}

Este trabajo consta de cuatro partes destacadas. La primera de ellas agrupa dos capítulos dedicados al estudio de ciertos esquemas en grupos y sus álgebras de Lie. Algunos de ellos son generalizaciones del grupo de Virasoro ([34, 31]), otros son generalizaciones de la versión algebraica de loop group de $\mathcal{S}^{1}([42,3])$, y los restantes pueden obtenerse como extensiones de estos esquemas en grupos. Además, estos últimos resultan ser grupos de automorfismos semilineales de determinados cuerpos de desarrollos de Laurent y sus álgebras de Lie se revelan, de manera natural, como álgebras de operadores diferenciales de orden uno. Cabe también destacar que el grupo de Weyl puede entenderse como un grupo de automorfismos en un cuerpo de desarrollos de Laurent.

La segunda parte engloba tres capítulos (numerados del 3 al 5), en los que se realiza un estudio sistemático del morfismo de Krichever para diferentes espacios de moduli. El primero de estos capítulos (el número 3) lo hemos destinado al moduli de fibrados vectoriales y curvas, y detalla los resultados expuestos en el punto 1; el capítulo 4 estudia el moduli de pares de Higgs con trivialización formal sobre una curva lisa fija (resultados del punto 3); y en el capítulo 5 entramos de lleno en la búsqueda de otro sistema integrable análogo al de Hitchin, detallando los resultados del punto 4 anterior.

La tercera parte es un estudio infinitesimal de los espacios de moduli tratados, y tiene dos capítulos (6 y 7) bien diferenciados. En el primero nos dedicamos a calcular los espacios tangentes a los espacios de moduli (resultados 2 y 5), computándolos desde dos puntos de vista: con técnicas clásicas de cohomología e hipercohomología y con las sencillas herramientas algebraicas que nos sugiere el morfismo de Krichever. Justificamos en este capítulo el estudio de los esquemas en grupos y sus álgebras de Lie realizado en la primera parte, pues, como ya hemos comentado, resulta que dichos grupos hacen las veces de generadores locales para los espacios de moduli (en el sentido de que actúan en ellos y el morfismo órbita es epiyectivo a nivel de espacios tangentes). En el capítulo 7 ofrecemos el último de los resultados (punto 6): cálculo explícito de la jerarquía de ecuaciones que describen el espacio de moduli de pares de Higgs con trivializaciones formales. 
Finalmente, en la cuarta parte hemos incluido cuatro apéndices, el primero dedicado a la construcción algebraica de la Grassmanniana de Sato, el segundo orientado a detallar la construcción inversa del morfismo de Krichever para el moduli de curvas, el tercero, que resume algunos resultados acerca de la teoría de operadores diferenciales desarrollada por Grothedieck. El cuarto apéndice es un resumen en Inglés de los resultados de esta memoria. 


\section{Parte I}

\section{Esquemas en grupos y sus álgebras de Lie.}





\section{Capítulo 1}

\section{Grupos formales.}

\subsection{Grupo de invertibles.}

Sea $k$ un cuerpo algebraicamente cerrado de característica nula.

Definición 1.1. ([3] ) Se define el functor contravariante:

$$
\begin{aligned}
\underline{k((z))^{*}: \mathcal{C}_{k-e s q}} & \rightarrow \mathcal{C}_{\text {grp }} \\
S & \mapsto \underline{k((z))^{*}}(S)=\mathrm{H}^{0}\left(S, \mathcal{O}_{S}\right)((z))^{*}
\end{aligned}
$$

donde para una $k$-álgebra $R, R((z))^{*}$ es el grupo de invertibles del anillo $R[[z]]\left[z^{-1}\right]$.

Usando los resultados de [3] puede verse que este functor es representable por un $k$ esquema formal en grupos cuya componente conexa del origen denotamos $\Gamma$, y donde la ley de grupo es la multiplicación de series de Laurent. Además podemos dar una descomposición:

$$
\Gamma=\Gamma^{-} \times \mathbb{G}_{m} \times \Gamma^{+}
$$

y $\gamma \in \Gamma(R)$ se escribe $\gamma=\left(\gamma_{-}, \gamma_{0}, \gamma_{+}\right)$con:

$$
\gamma_{-}=1+\sum_{j=-m}^{-1} a_{j} z^{j} \quad a_{j} \in \operatorname{Rad}(R)
$$

$\gamma_{0}$ invertible en $R$

$$
\gamma_{+}=1+\sum_{j \geq 1} a_{j} z^{j} \quad a_{j} \in R
$$

Observación 1.2. En [3] se calcula explítamente el representante del functor de invertibles $\underline{k((z))^{*}}$, que es:

$$
\varliminf_{l} \operatorname{Spf}\left(k\left\{\left\{x_{1}, x_{2}, \ldots\right\}\right\}\left[y_{0}, \frac{1}{y_{0}}, y_{1}, \ldots, y_{l}\right]\right)
$$


donde

$$
k\left\{\left\{x_{1}, x_{2}, \ldots\right\}\right\}:={\underset{\lim }{m}}_{m} k\left[x_{1}, \ldots, x_{m}\right] /\left(x_{1}, \ldots, x_{m}\right)^{m}
$$

parametriza los coeficientes nilpotentes (los de $\left.\Gamma^{-}\right), k\left[y_{0}, \frac{1}{y_{0}}\right]$ los invertibles (los de $\mathbb{G}_{m}$ ) y $\lim _{l} k\left[y_{1}, \ldots, y_{l}\right]$ los restantes (los de $\Gamma^{+}$).

Sea $V$ una $k((z))$-álgebra separable de dimensión $n$ y:

$$
V \simeq V_{1} \times \cdots \times V_{r}
$$

una descomposición en $k((z))$-álgebras irreducibles. Como en [31] se tiene:

$$
\Gamma_{V}=\Gamma_{V_{1}} \times \cdots \times \Gamma_{V_{r}} \quad \text { con } \quad \Gamma_{V_{i}}=\Gamma_{V_{i}}^{-} \times \mathbb{G}_{m} \times \Gamma_{V_{i}}^{+}
$$

De otro modo:

$$
\Gamma_{V}=\Gamma_{V}^{-} \times \mathbb{G}_{m}^{r} \times \Gamma_{V}^{+}, \text {donde } \quad \Gamma_{V}^{-}=\Gamma_{V_{1}}^{-} \times \cdots \times \Gamma_{V_{r}}^{-} \quad \text { y } \quad \Gamma_{V}^{+}=\Gamma_{V_{1}}^{+} \times \cdots \times \Gamma_{V_{r}}^{+}
$$

Observación 1.3. Como en la Observación 1.2 y siguiendo los resultados de [3] y [31], se sigue que $\Gamma_{V}^{-}$es el espectro formal del anillo:

$$
k\left\{\left\{x_{1}^{(1)}, x_{2}^{(1)}, \ldots\right\}\right\} \hat{\otimes} \cdots \hat{\otimes} k\left\{\left\{x_{1}^{(r)}, x_{2}^{(r)}, \ldots\right\}\right\},
$$

donde

$$
k\left\{\left\{x_{1}^{(i)}, x_{2}^{(i)}, \ldots\right\}\right\}:={\underset{m}{\leftrightarrows}}_{\lim _{m}} k\left[\left[x_{1}^{(i)}, \ldots, x_{m}^{(i)}\right]\right]
$$

\subsection{Grupos de automorfismos.}

Podemos dotar a $V$ con una estructura de espacio linealmente topológico definiendo como entornos del origen los subespacios $\left\{z^{m} V^{+}\right\}_{m \in \mathbb{Z}}$, donde $V^{+}$es una $k((z))$-subálgebra de $V$ que fijamos a partir de ahora. Para cada $k$-esquema $S$, denotemos:

$$
V_{S}:=V \widehat{\otimes}_{k} \mathcal{O}_{S}={\underset{m}{m}}_{\lim _{m}}\left(V / z^{m} V^{+}\right) \otimes \mathcal{O}_{S}
$$

donde $\widehat{\otimes}$ representa el producto tensorial completado (respecto de la topología definida por $\left.\left\{z^{m} V^{+}\right\}_{m \in \mathbb{Z}}\right)$.

Definición 1.4. ([3])

- Un sub- $\mathcal{O}_{S}$-módulo $U$ es un entorno si existe un subespacio $B$ en la topología tal que $\widehat{B_{S}} \subset U$ y $U / \widehat{B_{S}}$ es localmente libre de tipo finito.

- Un automorfismo $g$ (de $\widehat{V_{S}}$ como $\mathcal{O}_{S}$-módulo) se dice bicontinuo si $g\left(\widehat{B_{S}}\right)$ y $g^{-1}\left(\widehat{B_{S}}\right)$ son entornos para todo $B$ en la topología. 
- Se define el grupo lineal, $\mathrm{Gl}_{k}(V)$, como el functor contravariante sobre la categoría de $k$-esquemas dado por:

$$
S \mapsto \mathrm{Gl}_{k}(V)(S):=\left\{\text { automorfismos bicontinuos de } V_{S} \text { como } \mathcal{O}_{S} \text {-módulo }\right\}
$$

Definición 1.5. Se define el grupo de automorfismos de $k$-álgebras de $V_{i}$ como el functor en grupos sobre la categoría de $k$-esquemas dado por:

$$
S \rightarrow \mathrm{G}_{V_{i}}(S):=\operatorname{Aut}_{H^{0}\left(S, \mathcal{O}_{S}\right)-a l g}\left(V_{i}\right)_{S}
$$

(se entiende automorfismos bicontinuos) donde la ley de grupo viene dada por la composición de automorfismos.

Observación 1.6. Nótese que $\mathrm{G}_{V_{i}} \simeq \Gamma_{V_{i}}$ como esquemas, pero no como grupos, pues la ley de grupo en $\mathrm{G}_{V_{i}}$ es la composición de series y en $\Gamma_{V_{i}}$ el producto de series. Se tiene además que $\mathrm{G}_{V_{i}}$ es un subgrupo de $\mathrm{Gl}(V)$ (véase [34]).

Denotemos $\mathrm{G}$ al representante el functor de automorfismos de $k$-álgebras de $k((z))$ (que es un $k$-esquema conexo, véase [34]) y consideremos $G \times \stackrel{r}{.} \times G$. El grupo simétrico de $r$ letras, $\mathcal{S}_{r}$, actúa en $G \times \stackrel{r}{\stackrel{r}{*}} \times G$ (como subgrupo de $\mathrm{Gl}_{k}\left(k((z))^{r}\right)$ ) del modo siguiente:

$$
\begin{aligned}
\mathcal{S}_{r} \times(G \times \stackrel{r}{.} \times G) & \rightarrow G \times \stackrel{r}{.} \times G \\
\left(\sigma,\left(g_{1}, \ldots, g_{r}\right)\right) & \mapsto\left(g_{\sigma(1)}, \ldots, g_{\sigma(r)}\right)
\end{aligned}
$$

De este modo podemos entender el producto semidirecto:

$$
(G \times \stackrel{r}{\cdots} \times G) \rtimes \mathcal{S}_{r}
$$

que no es sino el menor subgrupo de $\mathrm{Gl}_{k}\left(k((z))^{r}\right)$ que contiene a ambos subgrupos.

Definición 1.7. Definimos el grupo de automorfismos de $k$-álgebras de $V, \underline{A u t}_{k}(V)$, como el functor que a cada $k$-esquema $S$ asocia:

$$
S \mapsto \operatorname{Aut}_{k-a l g}(V)(S):=\operatorname{Aut}_{H^{0}\left(S, \mathcal{O}_{S}\right)-a l g} V_{S}
$$

Siguiendo los pasos de [32] y teniendo en cuenta la descomposición de $V$ en $k((z))$ álgebras irreducibles $V_{i}$, se tiene que $\underline{\operatorname{Aut}}_{k}(V)$ es representable por el $k$-esquema formal en grupos:

$$
\left(\mathrm{G}_{1} \times \cdots \times \mathrm{G}_{r}\right) \rtimes \mathcal{S}_{r}
$$

que denotaremos $\mathrm{G}_{V}$. En particular esto dice que la componente conexa del origen (dada por la permutación trivial) es isomorfa a:

$$
\mathrm{G}_{1} \times \cdots \times \mathrm{G}_{r}
$$


Definición 1.8. La estructura de $k((z))$-álgebra de $V, \pi: k((z)) \rightarrow V$, nos permite definir un subfunctor en grupos de $\mathrm{G}_{V}$ :

$$
\mathrm{G}_{k((z))}^{V} \hookrightarrow \mathrm{G}_{V}
$$

como sigue:

$$
\mathrm{G}_{k((z))}^{V}:=\{\text { automorfismos de } V \text { que inducen un automorfismo de } k((z))\}
$$

de modo más explícito, automorfismos $\bar{g} \in \mathrm{G}_{V}$ haciendo conmutativo el siguiente diagrama:

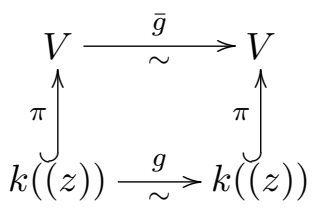

con $g \in \mathrm{G}_{k((z))} \stackrel{\text { not }}{=} \mathrm{G}$. El elemento de $\mathrm{G}_{k((z))}^{V}$ dado por el diagrama se denotará $\bar{g}$.

Observación 1.9. Esta definición es ligeramente distinta a la dada en [32, Sec.5], allí se define este grupo como un subgrupo de la componente conexa del origen de $\mathrm{G}_{V}$ y se pierde, por tanto, la combinatoria del grupo $\mathcal{S}_{r}$, que en nuestro trabajo necesitamos mantener para resultados posteriores.

Otra diferencia ciertamente significativa es que en [32, 5.4] se fija una estructura de $k((z))$-álgebra en $V$ concreta, mientras que nosotros vamos a permitir que varíe. Veamos qué es lo que queremos decir con esto.

Para el resto del trabajo supondremos que $V$ es una $k((z))$-álgebra separable de dimensión $n$, fijaremos una partición $\underline{n}=\left(n_{1}, \ldots, n_{r}\right)\left(\operatorname{con} n_{1}+\cdots+n_{r}=n\right)$ y utilizaremos las siguientes notaciones:

- Denotaremos $V_{\underline{n}}$ al producto $k\left(\left(z_{1}\right)\right) \times \cdots \times k\left(\left(z_{r}\right)\right)$ con la siguiente estructura de $k((z))$-álgebra:

$$
\begin{aligned}
k((z)) & \rightarrow k\left(\left(z_{1}\right)\right) \times \cdots \times k\left(\left(z_{r}\right)\right) \\
z & \mapsto\left(z_{1}^{n_{1}}, \ldots, z_{r}^{n_{r}}\right)
\end{aligned}
$$

(esta es la estructura usada en [32, 5.4]). Para abreviar, el grupo $\mathrm{G}_{k((z))}^{V_{n}}$ se denotará por $\mathrm{G}_{\underline{\underline{n}}}$.

- Sea $p(T) \in k[[z]][T]$ un polinomio mónico de grado $n$ de modo que su descomposicón en factores irreducibles es:

$$
p(T)=p_{1}(T) \cdots \cdots p_{r}(T), \text { donde } p_{i}(T)=T^{n_{i}}-z u_{i}(z, T)
$$

y $u_{i}(z, T) \in k[[z]][T]$ son polinomios de grado menor que $n_{i}$ tales que existe $u_{i}(z, T)^{-1}$ y $u_{i}(0,0)=1$. 
Denotaremos $V_{\underline{n}, p}$ al producto $\prod_{i=1}^{r} k((z))[T] / p_{i}(T)$ con la siguiente estructura de $k((z))$-álgebra:

$$
\begin{aligned}
k((z)) & \rightarrow k((z))[T] / p_{1}(T) \times \cdots \times k((z))[T] / p_{r}(T) \\
z & \mapsto\left(T^{n_{1}} u_{1}(z, T)^{-1}, \ldots, T^{n_{r}} u_{r}(z, T)^{-1}\right)
\end{aligned}
$$

(estas descomposiciones vienen motivadas por hechos geométricos, véase la Proposición 5.3 y la Observación 5.4. Para abreviar, denoteremos $\mathrm{G}_{k((z))}^{V_{n, p}}$ por $\mathrm{G}_{\underline{n}, p}$.

- Reservaremos la notación $V$ para los casos en los que, o bien no sea necesaria la estructura de $k((z))$-álgebra de $V$, o bien fijar la misma sea irrelevante.

Proposición 1.10. Se tiene un isomorfismo natural de functores en grupos:

$$
\mathrm{G}_{\underline{n}} \stackrel{\sim}{\longrightarrow} \mathrm{G}_{\underline{n}, p}
$$

Demostración. Basta demostrarlo para $\underline{n}=(n)$. Sea $h$ el siguiente morfismo de $V_{n}$ a $V_{n, p}$ :

$$
\begin{aligned}
h: k((z))[T] / T^{n}-z & \rightarrow k((z))[T] / T^{n}-z u(z, T) \\
T & \mapsto T u(z, T)^{-1 / n} \\
z & \mapsto z
\end{aligned}
$$

Se tiene que $h$ es un isomorfismo de $k((z))$-álgebras, por lo que el morfismo:

$$
\begin{aligned}
f_{h}: \mathrm{G}_{\underline{n}} & \rightarrow \mathrm{G}_{\underline{n}, p} \\
\bar{g}_{n} & \mapsto h \circ \bar{g}_{n} \circ h^{-1}
\end{aligned}
$$

está bien definido y es un isomorfismo de grupos. Además, el hecho de suponer que $u(0,0)=$ 1 implica que $u(0,0)^{-1}=1$ y por lo tanto escogemos $u(z, T)^{-1 / n}$ de modo que $u(0,0)^{-1 / n}=$ 1 . En este sentido, hay un isomorfismo $h$ distiguido y decimos que la elección de $f_{h}$ es natural.

Observación 1.11. Si en vez de trabajar sobre $k$ decidimos hacerlo directamente sobre un $k$-esquema $S$, el resultado anterior (el isomorfismo $h$ ) depende de la existencia de raíces $n_{i}$-ésimas en $\mathcal{O}_{S}$, y por lo tanto, la proposición es cierta si consideramos en la categoría de $S$-esquemas la topología étale (este tipo de topologías en categorías se llaman topologías de Grothendieck).

Sea Tr: $V \rightarrow k((z))$ el morfismo traza de $V$ como $k((z))$-álgebra finita. El morfismo $\pi: k((z)) \rightarrow V$ define una sección de $\operatorname{Tr}$ de modo que $\operatorname{Tr} \circ \tau=n \operatorname{Id}$ (donde $n$ es la dimensión $V$ sobre $k((z)))$ y por lo tanto, un elemento $a \in k((z))$ viene caracterizado por verificar $\operatorname{Tr}(a)=n a$. Se tiene entonces una descomposición de $k$-espacios vectoriales (no de $k$-álgebras):

$$
V \simeq k((z)) \oplus \operatorname{ker} \operatorname{Tr}
$$


Proposición 1.12. Sea $\bar{g} \in \mathrm{G}_{V}$. Entonces:

$$
\bar{g} \in \mathrm{G}_{k((z))}^{V} \Longleftrightarrow \operatorname{Tr} \circ \bar{g}=g \circ \operatorname{Tr}
$$

Demostración. Basta verlo para $V \simeq k((z))[T] / p(T)$ donde $p(T)=T^{n}-a_{1} T^{n-1}+\cdots+$ $(-1)^{n} a_{n}$ es un polinomio mónico e irreducible con coeficientes en $k((z))$.

En primer lugar, veamos que:

$$
\bar{g} \in \mathrm{G}_{k((z))}^{V} \Longleftrightarrow(\operatorname{Tr} \circ \bar{g}-g \circ \operatorname{Tr})_{\mid k((z))}=0
$$

En efecto, si $\bar{g} \in \mathrm{G}_{k((z))}^{V}$, por definición es $\bar{g} \circ \pi-\pi \circ g=0$. Componiendo con la aplicación $\operatorname{Tr}$ por la izquierda y teniendo en cuenta que $\operatorname{Tr} \circ \pi=n$ se deduce que $(\operatorname{Tr} \circ \bar{g}-g \circ \operatorname{Tr})_{\mid k((z))}=$ 0 . De modo análogo se demuestra el sentido opuesto.

El recíproco del enunciado resulta ahora de (1.2.6. Para el directo, teniendo en cuenta que el morfismo $\operatorname{Tr}$ es $k((z))$-lineal, basta ver que $(\operatorname{Tr} \circ \bar{g})\left(T^{i}\right)=(g \circ \operatorname{Tr})\left(T^{i}\right)$ para todo $\bar{g} \in \mathrm{G}_{k((z))}^{V}$. Ahora bien, dado $\bar{g} \in \mathrm{G}_{k((z))}^{V}$, como $p(T)=0$ en $V$, entonces $\bar{g}(p(T))=0$, por lo tanto:

$$
0=\bar{g}(p(T)) \stackrel{\bar{g} \in \mathrm{G}_{k((z))}^{V}}{=} \bar{g}\left(T^{n}\right)+\sum_{i=1}^{n}(-1)^{i} g\left(a_{i}\right) \bar{g}\left(T^{i}\right) \stackrel{\bar{g} \in \mathrm{G}_{V}}{=} \bar{g}(T)^{n}+\sum_{i=1}^{n}(-1)^{i} g\left(a_{i}\right) \bar{g}(T)^{i},
$$

(recuérdese que $\bar{g}(1)=1$ ) de donde deducimos que $g\left(a_{i}\right)$ son los coeficientes característicos de la homotecia multiplicar por $\bar{g}(T)$ en $V$ (recordemos que estos coeficientes característicos vienen dados por la fórmula $g\left(a_{i}\right)=\operatorname{Tr}\left(\wedge^{i} \bar{g}(T)\right)$ ).

Utilizando las fórmulas de Newton-Girard (véase [25, I.2]) (que expresan $\operatorname{Tr}\left(\bar{g}\left(T^{i}\right)\right)$ en función de los coeficientes característicos $\left.g\left(a_{i}\right)\right)$ :

$$
\operatorname{Tr}\left(\bar{g}\left(T^{i}\right)\right)=\left|\begin{array}{cccccc}
g\left(a_{1}\right) & 1 & 0 & \cdot & \cdot & \cdot \\
2 g\left(a_{2}\right) & g\left(a_{1}\right) & 1 & 0 & \cdot & \cdot \\
\cdot & \cdot & \cdot & \cdot & \cdot & \cdot \\
\cdot & \cdot & & \cdot & & \\
\cdot & \cdot & & & \cdot & \\
i g\left(a_{i}\right) & g\left(a_{i-1}\right) & \cdot & \cdot & \cdot & g\left(a_{1}\right)
\end{array}\right| \in k((z)), \quad \forall i \geq 0
$$

(donde $g\left(a_{i}\right)=0$ para $i>n$ ) y teniendo en cuenta que $g$ es un automorfismo de $k$-álgebras de $k((z))$, concluimos que:

$$
\operatorname{Tr}\left(\bar{g}\left(T^{i}\right)\right)=g\left|\begin{array}{cccccc}
a_{1} & 1 & 0 & \cdot & \cdot & \cdot \\
2 a_{2} & a_{1} & 1 & 0 & \cdot & \cdot \\
\cdot & \cdot & \cdot & \cdot & \cdot & \cdot \\
\cdot & \cdot & & \cdot & \\
\cdot & \cdot & & & \cdot \\
i a_{i} & a_{i-1} & \cdot & \cdot & \cdot & a_{1}
\end{array}\right|=g\left(\operatorname{Tr}\left(T^{i}\right)\right)
$$


Observación 1.13. Esta proposicón generaliza el Lema 5.8 de [32], pues permite variar la estructura de $k((z))$-álgebra de $V$.

Proposición 1.14. Se tiene una sucesión exacta de functores en grupos:

$$
0 \longrightarrow \operatorname{Aut}_{k((z))-\text { alg }} V \longrightarrow \mathrm{G}_{k((z))}^{V} \stackrel{p}{\longrightarrow} \mathrm{G} \longrightarrow 0
$$

donde $p(\bar{g})=g$.

Demostración. Veamos primero que el núcleo de $p$ es el grupo de automorfismos de $k((z))$ álgebras de $V$. En efecto:

$$
\begin{aligned}
\bar{g} \in \operatorname{ker} p & \Longleftrightarrow g(z)=z \Longleftrightarrow \bar{g}(z v)=g(z) \bar{g}(v)=z \bar{g}(v) \quad \forall v \in V \Longleftrightarrow \\
& \Longleftrightarrow \bar{g} \in \operatorname{Aut}_{k((z))-\text { alg }} V
\end{aligned}
$$

En el caso $\mathrm{G}_{k((z))}^{V}=\mathrm{G}_{\underline{n}}$, la epiyectividad se sigue de la propia definición. Para el resto, basta usar la topología étale (véase la Observación 1.11).

\subsubsection{Estudio en el caso de $V_{\underline{n}}$.}

Estudiemos primero el caso $\underline{n}=(n)$.

$$
\begin{aligned}
k((z)) & \rightarrow V_{n}=k\left(\left(z_{n}\right)\right):=k((z))[T] / T^{n}-z \\
z & \mapsto z=z_{n}^{n} .
\end{aligned}
$$

El grupo $\mu_{n}$ de las raíces $n$-ésimas de la unidad actúa en $V_{n}$ por la fórmula:

$$
\xi * q(T):=q(\xi T)
$$

de modo que para cada $\xi \in \mu_{n}$ el endomorfismo $k$-lineal:

$$
\bar{\xi}: V_{n} \rightarrow V_{n}
$$

definido por $\bar{\xi}(q(T)):=q(\xi T)$ es un automorfismo de $k$-álgebras, es decir, un punto de $\mathrm{G}_{V_{n}}$. Y no sólo eso, sino que como $\xi^{n}=1$ se tiene que:

$$
\bar{\xi}\left(T^{n}\right)=T^{n},
$$

o lo que es lo mismo, $\bar{\xi}(z)=z$. De aquí deducimos dos cosas:

- $\bar{\xi}$ define un elemento en $\operatorname{Aut}_{k((z))-a l g} V_{n}$.

- $\bar{\xi}$ define un automorfismo de $k$-álgebras en $k((z))$ que es la identidad, es decir, define un elemento del núcleo de la proyección:

$$
\pi: \mathrm{G}_{n} \rightarrow G \rightarrow 0
$$


Observemos además que el núcleo de $\pi$ está formado por los automorfismos $\bar{g}$ tales que $\bar{g}\left(T^{n}\right)=T^{n}$ luego:

$$
\bar{g} \in \mu_{n}
$$

por la misma razón es $\bar{g}(z)=z$, es decir:

$$
\bar{g} \in \operatorname{Aut}_{k((z))-a l g} V_{n} .
$$

En definitiva, se tiene un isomorfismo:

$$
\mu_{n} \simeq \operatorname{Aut}_{k((z))-a l g} V_{n}
$$

y por tanto, recuperamos la sucesión exacta:

$$
0 \rightarrow \mu_{n} \rightarrow G_{n} \rightarrow G \rightarrow 0
$$

de la Porposición 5.6 de [32].

Para el caso general $\underline{n}=\left(n_{1}, \ldots, n_{r}\right)$ :

$$
\begin{aligned}
k((z)) & \rightarrow V_{\underline{n}}=k\left(\left(z_{1}\right)\right) \times \cdots \times k\left(\left(z_{r}\right)\right) \\
z & \mapsto\left(z_{1}^{n_{1}}, \ldots, z_{r}^{n_{r}}\right)
\end{aligned}
$$

escribamos $V_{i}=k\left(\left(z_{i}\right)\right)=k((z))[T] / T^{n_{i}}-z$ y sea $\mu_{n_{i}}$ el grupo de las raíces $n_{i}$-ésimas de la unidad. Como antes, definimos una acción de $\mu_{n_{1}} \times \cdots \times \mu_{n_{r}}$ en $V$ :

$$
\begin{aligned}
\left(\mu_{n_{1}} \times \cdots \times \mu_{n_{r}}\right) \times\left(V_{1} \times \cdots \times V_{r}\right) & \rightarrow V_{1} \times \cdots \times V_{r} \\
\left(\left(\xi_{1}, \ldots, \xi_{r}\right),\left(q_{1}\left(T_{1}\right), \ldots, q_{r}\left(T_{r}\right)\right)\right) & \mapsto\left(q_{1}\left(\xi_{1} \cdot T_{1}\right), \ldots, q_{r}\left(\xi_{r} \cdot T_{r}\right)\right)
\end{aligned}
$$

Consideremos el grupo simétrico de $r$ letras, $\mathcal{S}_{r}$, y sea $\sigma \in \mathcal{S}_{r}$. Definimos la siguiente acción de $\mathcal{S}_{r}$ en $\mu_{\underline{n}}=\mu_{n_{1}} \times \cdots \times \mu_{n_{r}}$ :

$$
\sigma *\left(\xi_{1}, \ldots, \xi_{r}\right):=\left(\xi_{\sigma(1)}, \ldots, \xi_{\sigma(r)}\right) .
$$

Para que esta acción esté bien definida es necesario que para todo $i=1, \ldots, r$ sea:

$$
\xi_{\sigma(i)} \in \mu_{n_{i}} .
$$

Definimos entonces el subgrupo $\mathcal{S}_{\underline{\underline{n}}}$ de $\mathcal{S}_{r}$ como aquel generado por las transposiciones $(i, j) \in \mathcal{S}_{r}$ tales que $n_{i}=n_{j}$, es decir:

$$
\mathcal{S}_{\underline{n}}=<\left\{(i, j) \in \mathcal{S}_{r} \mid n_{i}=n_{j}\right\}>
$$

Se tiene entonces una acción bien definida de $\mathcal{S}_{\underline{n}}$ en $\mu_{\underline{n}}$ que nos permite definir el producto semidirecto:

$$
\mu_{\underline{n}} \rtimes \mathcal{S}_{\underline{n}} .
$$

En definitiva, queda probada la siguiente: 
Proposición 1.15. Se tiene una sucesión exacta de esquemas formales en grupos:

$$
0 \longrightarrow \operatorname{Aut}_{k((z))-a l g} V_{\underline{n}} \longrightarrow \mathrm{G}_{\underline{\underline{n}}} \stackrel{\pi}{\longrightarrow} \mathrm{G} \longrightarrow 0
$$

y un isomorfismo:

$$
\operatorname{Aut}_{k((z))-\text { alg }} V_{\underline{n}} \simeq \mu_{\underline{n}} \rtimes \mathcal{S}_{\underline{n}}
$$

Observación 1.16. Si tomamos $V=k((z))^{n}$ con la estructura trivial de $k((z))$-álgebra, los elementos $\bar{g} \in \mathrm{G}_{k((z))}^{V}$ se escriben:

$$
\bar{g}=(g, . . . ., g) \quad g \in \mathrm{G} .
$$

Por lo tanto $\mathrm{G}_{k((z))}^{V}$ coincide, en este caso, con la imagen de $\mathrm{G}$ en $\mathrm{G} \times \stackrel{n}{ } \times \times G$ por la inclusión diagonal.

\subsection{Grupo de Heisenberg y grupo de Weyl.}

Sea $\underline{V}$ el functor que a cada $k$-esquema $S$ asocia $V(S)$ y $\mathrm{Gl}_{k((z))}(V)$ el functor en grupos ("loop group") que a cada $k$-esquema $S$ asocia los automorfismos de $\mathrm{H}^{0}\left(S, \mathcal{O}_{S}\right)((z))$ módulos de $V(S)$ (nótese que para $n=1$ es precisamente el functor de invertibles $\left.k((z))^{*}\right)$. Es un subfunctor en grupos de $\mathrm{Gl}_{k}(V)$. Este functor es representable por un esquema formal en grupos que denotaremos igual y tiene tres subgrupos distinguidos $\mathrm{Gl}_{k((z))}(V)^{-}, \mathrm{Gl}(n, k)$, $\mathrm{Gl}_{k((z))}(V)^{+}$de modo que la "big cell":

$$
\mathrm{Gl}_{k((z))}(V)^{0}=\mathrm{Gl}_{k((z))}(V)^{-} \cdot \mathrm{Gl}(n, k) \cdot \mathrm{Gl}_{k((z))}(V)^{+}
$$

es un subesquema abierto de la componente conexa del origen de $\mathrm{Gl}_{k((z))}(V)$ (véase [38, Ch. 8.1.2], [6, Prop. 1.11]).

La acción por homotecias de $\underline{V}^{*}$ en $\underline{V}$ induce una inclusión de functores en grupos:

$$
\underline{V}^{*} \hookrightarrow \mathrm{Gl}_{k((z))}(V) .
$$

Definición 1.17. Se define el functor en grupos de Heisenberg, Heis ${ }_{V}$, como la imagen de $\Gamma_{V}$ por la inclusión anterior (recuérdese que $\Gamma_{V}$ es la componente conexa del origen del representante del functor $\left.\underline{V}^{*}\right)$.

Definición 1.18. Se define el functor en grupos de Weyl como el cociente:

$$
\mathcal{W}=\mathrm{N}_{k((z))}\left(\text { Heis }_{V}\right) / \text { Heis }_{V}
$$

siendo $\mathrm{N}_{k((z))}\left(\operatorname{Heis}_{V}\right)$ el normalizador de $\operatorname{Heis}_{V}$ en $\mathrm{Gl}_{k((z))}(V)$.

Teorema 1.19. Se tiene que: 
1. La siguiente sucesión es exacta:

$$
0 \rightarrow \operatorname{Heis}_{V} \rightarrow \mathrm{N}_{k((z))}\left(\operatorname{Heis}_{V}\right) \rightarrow \operatorname{Aut}_{k((z))-a l g} V \rightarrow 0
$$

2. $\mathcal{W} \simeq \operatorname{Aut}_{k((z))-a l g} V$.

3. La acción por conjugación de $\operatorname{Aut}_{k((z))-a l g} V$ en $\mathrm{Heis}_{V}$ induce un isomorfismo:

$$
\mathrm{N}_{k((z))}\left(\operatorname{Heis}_{V}\right) \simeq \operatorname{Heis}_{V} \rtimes \mathcal{W}
$$

Demostración. Consideremos la sucesión exacta:

$$
0 \rightarrow \operatorname{Heis}_{V} \rightarrow \mathrm{N}_{k((z))}\left(\operatorname{Heis}_{V}\right) \rightarrow \mathrm{N}_{k((z))}\left(\text { Heis }_{V}\right) / \text { Heis }_{V} \rightarrow 0 .
$$

Observemos en primer lugar que si $g \in \mathrm{N}_{k((z))}\left(\operatorname{Heis}_{V}\right)$, en cada órbita $g$ Heis $_{V}$ existe un único elemento tal que $g(1)=1$, pues basta considerar la acción de Heis $V$ en $\mathrm{N}_{k((z))}\left(\operatorname{Heis}_{V}\right)$ :

$$
\rho_{v} * g:=\rho_{v} \circ g=v \cdot g
$$

y tomar $v=g^{-1}(1)$, donde $\rho_{v} \in$ Heis $_{V}$ es la homotecia multiplicar por $v \in \Gamma_{V}$.

Veamos entonces que si $g \in \mathrm{N}_{k((z))}\left(\operatorname{Heis}_{V}\right)$ y $g(1)=1$ entonces $g \in \operatorname{Aut}_{k((z))-\text { alg }} V$ (el recíproco es inmediato). En efecto, como por definición $g$ es $k((z))$-lineal, es suficiente demostrar que si $v, w \in V$ entonces:

$$
g(v \cdot w)=g(v) \cdot g(w) .
$$

Pero esto se sigue diractemente de:

$$
g(v \cdot w)=\left(g \circ \rho_{v}\right)(w)=\left(\rho_{g(v)} \circ g\right)(w)=g(v) \cdot g(w) .
$$

De aquí se deducen 1 y 2 , esto es, la siguiente sucesión es exacta:

$$
0 \rightarrow \operatorname{Heis}_{V} \rightarrow \mathrm{N}_{k((z))}\left(\operatorname{Heis}_{V}\right) \stackrel{\pi}{\rightarrow} \operatorname{Aut}_{k((z))-\text { alg }} V \rightarrow 0
$$

e induce un isomorfismo $\mathcal{W} \simeq \operatorname{Aut}_{k((z))-\text { alg }} V$.

Para probar 3, basta observar que la inclusión natural:

$$
\operatorname{Aut}_{k((z))-a l g} V \hookrightarrow \mathrm{N}_{k((z))}\left(\text { Heis }_{V}\right)
$$

(Aut $_{k((z))-a l g} V$ actúa en $\mathrm{Heis}_{V}$ por conjugación) define una sección de grupos de $\pi$, por lo tanto la sucesión exacta rompe. En particular (véase [10, IV.6]):

$$
\mathrm{N}_{k((z))}\left(\operatorname{Heis}_{V}\right) \simeq \text { Heis }_{V} \rtimes \mathcal{W}
$$

donde la ley de grupo de $\operatorname{Heis}_{V} \rtimes \mathcal{W}$ es:

$$
\left(\rho_{v}, g\right) *\left(\rho_{v^{\prime}}, g^{\prime}\right)=\left(\rho_{v} \circ c_{g}\left(\rho_{v^{\prime}}\right), g \circ g^{\prime}\right)
$$

siendo $c_{g}\left(\rho_{v^{\prime}}\right)=g \circ \rho_{v^{\prime}} \circ g^{-1}$ la acción de $\operatorname{Aut}_{k((z))-a l g} V$ en Heis $V$. 


\subsection{Grupos de automorfismos semilineales.}

Definición 1.20. Se define el functor en grupos $\operatorname{SGl}_{k((z))}(V)$ de los automorfismos semilineales de $V$ como el functor de automorfismos $k$-lineales:

$$
\gamma: V \simeq V
$$

para los que existe un automorfismo de $k$-álgebras de $k((z)), g \in \mathrm{G}$, de modo que:

$$
\gamma(z \cdot v)=g(z) \cdot \gamma(v)
$$

Proposición 1.21. Se tiene una sucesión exacta de functores en grupos:

$$
0 \rightarrow \mathrm{Gl}_{k((z))}(V) \rightarrow \mathrm{SGl}_{k((z))}(V) \rightarrow \mathrm{G} \rightarrow 0
$$

de modo que la acción de $\mathrm{G}$ en $\mathrm{Gl}_{k((z))}(V)$ induce un isomorfismo:

$$
\operatorname{SGl}_{k((z))}(V) \simeq \mathrm{Gl}_{k((z))}(V) \rtimes \mathrm{G}
$$

Demostración. Veamos en primer lugar la epiyectividad. Sea $g \in \mathrm{G}$ y fijemos una identificación $V \simeq k^{n} \otimes_{k} k((z))$. Se tiene entonces que:

$$
\operatorname{Id} \otimes g: V \simeq k^{n} \otimes_{k} k((z)) \simeq k^{n} \otimes_{k} k((z)) \simeq V
$$

es un elemento de $\operatorname{SGl}_{k((z))}(V)$, de donde se sigue la epiyectividad. El núcleo de:

$$
\mathrm{SGl}_{k((z))}(V) \rightarrow \mathrm{G} \rightarrow 0
$$

es $\mathrm{Gl}_{k((z))}(V)$, pues son automorfismos $\gamma \in \operatorname{SGl}_{k((z))}(V)$ tales que el automorfismo $g \in G$ que definen es la identidad, o sea, aquellos que verifican:

$$
\gamma(z \cdot v)=z \cdot \gamma(v)
$$

lo que significa que son $k((z))$-lineales.

Para ver que $\mathrm{SGl}_{k((z))}(V)=\mathrm{Gl}_{k((z))}(V) \rtimes \mathrm{G}$ observemos que la extensión:

$$
0 \rightarrow \mathrm{Gl}_{k((z))}(V) \rightarrow \mathrm{SGl}_{k((z))}(V) \rightarrow \mathrm{G} \rightarrow 0
$$

tiene núcleo no abeliano y por tanto define un morfismo de grupos:

$$
\psi: \mathrm{G} \rightarrow \operatorname{Out}\left(\mathrm{Gl}_{k((z))}(V)\right)
$$

(donde Out $\left(\mathrm{Gl}_{k((z))}(V)\right)$ denota el grupo de automorfismos externos de $\mathrm{Gl}_{k((z))}(V)$, es decir, el cociente de los automorfismos por los automorfismos internos). En particular, no tendremos el producto semidirecto $\operatorname{SGl}_{k((z))}=\mathrm{Gl}_{k((z))}(V) \rtimes \mathrm{G}$ salvo si $\psi$ levanta a un morfismo de grupos:

$$
\tilde{\psi}: \mathrm{G} \rightarrow \operatorname{Aut}\left(\mathrm{Gl}_{k((z))}(V)\right)
$$


(véase [10, Capítulo IV.6]). Sea entonces $s: \mathrm{G} \rightarrow \mathrm{SGl}_{k((z))}(V)$ una sección de conjuntos de:

$$
\operatorname{SGl}_{k((z))}(V) \rightarrow \mathrm{G} \rightarrow 0,
$$

$\psi$ levanta a un morfismo de conjuntos:

$$
\begin{aligned}
\tilde{\psi}: \mathrm{G} & \rightarrow \operatorname{Aut}\left(\mathrm{Gl}_{k((z))}(V)\right) \\
g & \mapsto\left\{\gamma \mapsto s(g) \circ \gamma \circ s(g)^{-1}\right\}
\end{aligned}
$$

que es morfismo de grupos si y sólo si la sección $s$ es de grupos. Ahora bien, dado $g \in \mathrm{G}$ la sección definida por $s(g)=\operatorname{Id} \otimes g$ es una sección de grupos, lo que implica que en nuestro caso $\tilde{\psi}$ es de grupos y por lo tanto:

$$
\operatorname{SGl}_{k((z))}(V)=\mathrm{Gl}_{k((z))}(V) \rtimes \mathrm{G} .
$$

Observación 1.22. En particular, para $V=k((z))$ el grupo $\mathrm{SGl}_{k((z))}(k((z)))$ es exactamente el grupo $\Gamma \rtimes \mathrm{G}$ utilizado en [32, Section 6].

Definición 1.23. Se define el functor en grupos $\mathrm{SHeis}_{V}$ como el subfunctor de $\mathrm{SGl}_{k((z))}(V)$ formado por aquellos automorfismos $k$-lineales:

$$
\gamma: V \simeq V
$$

para los que existe un automorfismo de $k$-álgebras de $V, g \in \mathrm{G}_{V}$, y un $w \in V^{*}$ tales que:

$$
\gamma(v)=w \cdot g(v)
$$

El inverso de $\gamma \in$ SHeis $_{V}$ viene dado por:

$$
\gamma^{-1}(v)=\left(g^{-1}(w)\right)^{-1} \cdot g^{-1}(v)
$$

donde $\gamma^{-1}(1)=\left(g^{-1}(w)\right)^{-1}$.

A la postre se deduce que $w=\gamma(1) \in V^{*}$.

Proposición 1.24. Se tiene una sucesión exacta de functores en grupos:

$$
0 \rightarrow \text { Heis }_{V} \rightarrow \text { SHeis }_{V} \rightarrow \mathrm{G}_{V} \rightarrow 0
$$

de modo que la acción de $\mathrm{G}_{V}$ en HeisV induce un isomorfismo:

$$
\text { SHeis }_{V} \simeq \text { Heis }_{V} \rtimes \mathrm{G}_{V}
$$

Demostración. Se demuestra de modo análogo a la Proposición 1.21. Cabe destacar que en este caso es sencillo ver que:

$$
\mathrm{G}_{V}:=\left\{\gamma \in \text { SHeis }_{V} \mid \gamma(1)=1\right\} .
$$


Proposición 1.25. La inclusión natural $\mathrm{G}_{V} \hookrightarrow \mathrm{N}_{k}\left(\right.$ Heis $\left._{V}\right)$ induce un isomorfismo:

$$
\mathrm{N}_{k}\left(\text { Heis }_{V}\right) / \text { Heis }_{V} \simeq \mathrm{G}_{V}
$$

donde $\mathrm{N}_{k}\left(\mathrm{Heis}_{V}\right)$ es el normalizador de $\mathrm{Heis}_{V}$ en $\mathrm{Gl}_{k}(V)$.

Demostración. Se sigue directamente de la demostración de la proposición 1.19

Corolario 1.26. Se tiene un isomorfismo:

$$
\text { SHeis }_{V} \simeq \mathrm{N}_{k}\left(\text { Heis }_{V}\right)
$$

Demostración. En virtud de las proposiciones $1.24 \mathrm{y} 1.25$ se tiene un diagrama:

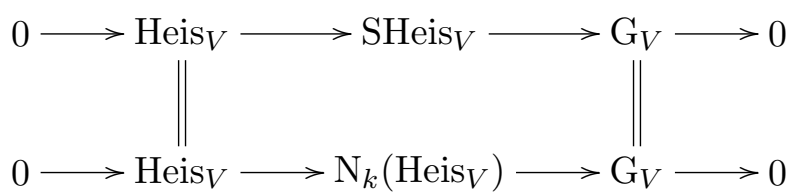

Basta entonces ver que existe un morfismo de grupos:

$$
\text { SHeis }_{V} \rightarrow \mathrm{N}_{k}\left(\text { Heis }_{V}\right) \text {. }
$$

En efecto, sea $\gamma \in$ SHeis $_{V}$ y $\rho_{w} \in$ Heis $_{V}$, entonces:

$$
\begin{aligned}
\gamma^{-1} \circ \rho_{w} \circ \gamma(v) & =\gamma^{-1}(w \gamma(1) g(v))=\gamma^{-1}(1) g^{-1}(w \gamma(1) g(v))= \\
& =\gamma^{-1}(1)\left(\gamma^{-1}(1)\right)^{-1} g^{-1}(w) v=g^{-1}(w) v=\rho_{g^{-1}(w)}(v),
\end{aligned}
$$

es decir, $\gamma \in \mathrm{N}_{k}\left(\right.$ Heis $\left._{V}\right)$.

Definición 1.27. Se define el functor en grupos $\operatorname{SHeis}_{k((z))}^{V}$ como el functor de automorfismos $k$-lineales:

$$
\gamma: V \simeq V
$$

para los que existe un automorfismo $\bar{g} \in \mathrm{G}_{k((z))}^{V}$ y un $w \in V^{*}$ de modo que:

$$
\gamma(v)=w \cdot \bar{g}(v)
$$

Proposición 1.28. Se tiene una sucesión exacta de functores en grupos:

$$
0 \rightarrow \operatorname{Heis}_{V} \rightarrow \operatorname{SHeis}_{k((z))}^{V} \rightarrow \mathrm{G}_{k((z))}^{V} \rightarrow 0
$$

de modo que la acción de $\mathrm{G}_{k((z))}^{V}$ en Heis $\mathrm{H}_{V}$ induce un isomorfismo:

$$
\operatorname{SHeis}_{k((z))}^{V} \simeq \operatorname{Heis}_{V} \rtimes \mathrm{G}_{k((z))}^{V}
$$


Demostración. Es consecuencia inmediata de 1.24

Observación 1.29. Este tipo de grupos pueden englobarse en construcciones aún más generales. Resumimos aquí algunos de los resultados e ideas en esta dirección.

Se tiene que:

$$
\mathrm{G}_{k((z))}^{V} \cap \mathrm{Gl}_{k((z))}(V)=\operatorname{Aut}_{k((z))-a l g} V
$$

(como subgrupos de $\mathrm{Gl}_{k}(V)$ ) y un diagrama conmutativo:

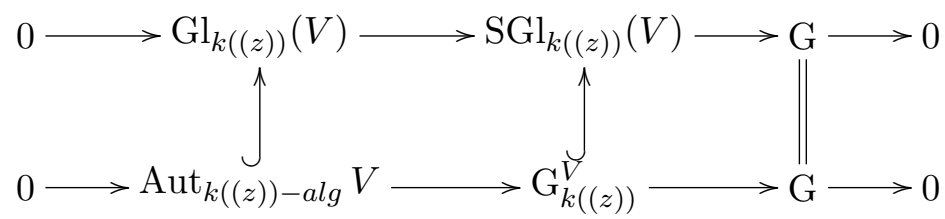

Donde la inclusión $\mathrm{G}_{k((z))}^{V} \hookrightarrow \operatorname{SGl}_{k((z))}(V)$ se sigue de la propia definición de $\mathrm{G}_{k((z))}^{V}$ (Definición 1.8).

Puede verse así mismo que se tiene una inclusión de subfunctores en grupos:

$$
G_{k((z))}^{V} \hookrightarrow \mathrm{N}_{k}\left(\mathrm{Gl}_{k((z))}(V)\right)
$$

(donde $\mathrm{N}_{k}\left(\mathrm{Gl}_{k((z))}(V)\right)$ es el normalizador de $\mathrm{Gl}_{k((z))}(V)$ en $\mathrm{Gl}_{k}(V)$ ) que nos permite dar una acción de $\mathrm{G}_{k((z))}^{V}$ en $\mathrm{Gl}_{k((z))}(V)$ por la fórmula:

$$
\begin{aligned}
\mathrm{G}_{k((z))}^{V} \times \mathrm{Gl}_{k((z))}(V) & \rightarrow \mathrm{Gl}_{k((z))}(V) \\
(g, \gamma) & \mapsto c_{g}(\gamma)=g^{-1} \circ \gamma \circ g
\end{aligned}
$$

Se define entonces el functor:

$$
\mathrm{G}_{k((z))}^{V} \ltimes \mathrm{Gl}_{k((z))}(V)
$$

como el menor subfunctor en grupos de $\mathrm{Gl}_{k}(V)$ que contiene a ambos, equivalentemente, el subfunctor en grupos de $\mathrm{Gl}_{k}(V)$ generado por ambos. La ley de grupo viene dada por:

$$
\left(g_{1}, \gamma_{1}\right) *\left(g_{2}, \gamma_{2}\right)=\left(g_{1} \circ g_{2}, c_{g_{2}}\left(\gamma_{1}\right) \circ \gamma_{2}\right),
$$

y el inverso de $(g, \gamma)$ es $\left(g^{-1}, c_{g^{-1}}\left(\gamma^{-1}\right)\right)$.

En particular, si en vez de tomar $\mathrm{Gl}_{k((z))}(V)$ tomamos el subgrupo Heis $V$ (imagen del grupo $\Gamma_{V}$ de invertibles en $V$ ), la ley de grupo se simplifica:

$$
\left(g_{1}, \gamma_{1}\right) *\left(g_{2}, \gamma_{2}\right)=\left(g_{1} \circ g_{2}, g_{2}^{-1}\left(\gamma_{1}\right) \cdot \gamma_{2}\right)
$$

y cuando $V=k((z))$ obtenemos la ley de grupo de $\Gamma \rtimes \mathrm{G}$ dada en [32, Section 6]. 
Por último, puede demostrarse que se tiene una sucesión exacta:

$$
0 \longrightarrow \operatorname{Aut}_{k((z))-a l g} V \stackrel{i}{\longrightarrow} \mathrm{G}_{k((z))}^{V} \ltimes \mathrm{Gl}_{k((z))}(V) \stackrel{p}{\longrightarrow} \operatorname{SGl}_{k((z))}(V) \longrightarrow 0
$$

que induce un isomorfismo:

$$
\mathrm{G}_{k((z))}^{V, 0} \ltimes \mathrm{Gl}_{k((z))}(V) \simeq \operatorname{SGl}_{k((z))}(V),
$$

donde $\mathrm{G}_{k((z))}^{V, 0}$ denota la componente conexa del origen de $\mathrm{G}_{k((z))}^{V}$, que por la proposición 1.14 es isomorfa a $\mathrm{G}$. 


\section{Capítulo 2}

\section{Álgebras de Lie de los grupos formales.}

Sea $k$ un cuerpo algebraicamente cerrado de característica nula y $V$ una $k((z))$-álgebra finita y separable de dimensión $n$.

Sea $\mathrm{Gl}_{k}(V)$ el functor en grupos de los automorfismos de $k$-espacios vectoriales de $V$, definimos su álgebra de Lie por:

$$
\operatorname{End}_{k} V:=\mathrm{Gl}_{k}(V)(k[\epsilon]) \times \mathrm{Gl}_{k}(V)(k)\{\mathrm{Id}\}
$$

donde denotamos $k[\epsilon]$ al anillo de los números duales $k[\epsilon] / \epsilon^{2}$. Así mismo, definimos $\operatorname{End}_{k((z))} V$ como el álgebra de Lie de $\mathrm{Gl}_{k((z))}(V)$, es decir:

$$
\begin{gathered}
\operatorname{Gl}_{k((z))}(V)(k[\epsilon]) \times \mathrm{Gl}_{k((z))}(V)(k) \\
\gamma=\operatorname{Id}_{n}+\epsilon \bar{\gamma} \mapsto \bar{\gamma}
\end{gathered}
$$

El paréntesis de Lie $\left[\bar{\gamma}_{1}, \bar{\gamma}_{2}\right]$ es el elemento que verifica:

$$
\left[\gamma_{1}, \gamma_{2}\right]:=\gamma_{1} \gamma_{2} \gamma_{1}^{-1} \gamma_{2}^{-1}=\operatorname{Id}_{n}+\epsilon_{1} \epsilon_{2}\left[\bar{\gamma}_{1}, \bar{\gamma}_{2}\right],
$$

donde $\gamma_{i}=\operatorname{Id}_{n}+\epsilon_{i} \bar{\gamma}_{i} \in \mathrm{Gl}_{k((z))}(V)\left(k\left[\epsilon_{i}\right]\right) \times_{\mathrm{Gl}_{k((z))}(V)(k)}\{\mathrm{Id}\}$. Explícitamente:

$$
\left[\bar{\gamma}_{1}, \bar{\gamma}_{2}\right]:=\bar{\gamma}_{1} \bar{\gamma}_{2}-\bar{\gamma}_{2} \bar{\gamma}_{1}
$$

En particular:

$$
\Gamma_{V}(k[\epsilon]) \times_{\Gamma_{V}(k)}\{\mathrm{Id}\}=V
$$

\section{1. Álgebra de Lie de los grupos de automorfismos.}


Proposición 2.1. Se tiene un isomorfismo de álgebras de Lie:

$$
\operatorname{Lie}\left(\mathrm{G}_{V}\right) \simeq \operatorname{Der}_{k}(V, V) .
$$

Demostración. Por definición tenemos que:

$$
\begin{aligned}
\text { Lie } \mathrm{G}_{V}: & \left.=\mathrm{G}\left(k[\epsilon] / \epsilon^{2}\right) \times_{\mathrm{G}(k)}\{\operatorname{Id}\}\right)= \\
& =\left\{g: V \oplus \epsilon V \simeq V \oplus \epsilon V \text { de } k[\epsilon] \text {-álgebras } \mid g_{\left.\right|_{\epsilon=0}}: V \stackrel{\mathrm{Id}}{\rightarrow} V\right\}
\end{aligned}
$$

es decir, $g=\mathrm{Id}+\epsilon g_{0}$. Como $g$ es de $k[\epsilon]$-álgebras, ha de ser:

$$
\begin{gathered}
g(1)=1 \Longrightarrow g_{0}(1)=0 \\
g\left(u u^{\prime}\right)=g(u) g\left(u^{\prime}\right) \Longrightarrow g_{0}\left(u u^{\prime}\right)=u g_{0}\left(u^{\prime}\right)+g_{0}(u) u^{\prime} .
\end{gathered}
$$

Lo que implica que $g_{0} \in \operatorname{Der}_{k}(V, V)$. Resumiendo, se tiene un isomorfismo de $k$-espacios vectoriales:

$$
\begin{aligned}
& \text { Lie } \mathrm{G}_{V} \simeq \operatorname{Der}_{k}(V, V) \\
& 1+\epsilon g_{0} \mapsto g_{0}
\end{aligned}
$$

Puede compromprobarse que los paréntesis de Lie coinciden (véase [34, Thm 3.5]).

Proposición 2.2. Si $V \simeq V_{1} \times \cdots \times V_{r}$ es producto directo de $k$-álgebras, entonces:

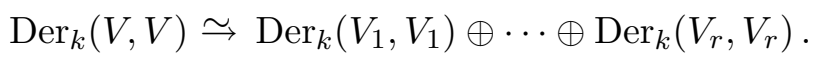

Demostración. Basta verlo para el caso $r=2$. Las proyecciones $V \rightarrow V_{i}$ inducen morfismos $\Omega_{V / k} \rightarrow \Omega_{V_{i} / k}$, con lo que se tienen un morfismo $\Omega_{V / k} \rightarrow \Omega_{V_{1} / k} \oplus \Omega_{V_{2} / k}$ que verifica $d\left(v_{1}, v_{2}\right)=\left(d v_{1}, d v_{2}\right)$, siendo $d$ la diferencial correspondiente en cada caso. Las inmersiones $V_{i} \rightarrow V$ dan lugar a un morfismo $\Omega_{V_{1} / k} \oplus \Omega_{V_{2} / k} \rightarrow \Omega_{V / k}$ que verifica $\left(d v_{1}, d v_{2}\right)=d\left(v_{1}, v_{2}\right)$ $\mathrm{y}$ es el inverso del anterior. Es decir, tenemos un isomorfismo:

$$
\Omega_{V / k} \simeq \Omega_{V_{1} / k} \oplus \Omega_{V_{2} / k} .
$$

Por otra parte tenemos un isomorfismo de $V$-módulos:

$$
\begin{aligned}
\operatorname{Hom}_{V}\left(\Omega_{V / k}, V\right) & \simeq \operatorname{Der}_{k}(V, V) \\
\eta & \mapsto \eta \circ d
\end{aligned}
$$

siendo $d$ la diferencial en $V$. De este modo:

$$
\operatorname{Der}_{k}(V, V) \stackrel{\sim}{\rightarrow} \operatorname{Hom}_{V_{1} \times V_{2}}\left(\Omega_{V_{1} / k} \oplus \Omega_{V_{2} / k}, V_{1} \times V_{2}\right)
$$

Sea ahora $D: \Omega_{V_{1} / k} \oplus \Omega_{V_{2} / k} \rightarrow V_{1} \times V_{2}$ un morfismo $V_{1} \times V_{2}$-lineal, se tiene entonces que:

$$
D\left(f_{1}, 0\right)=D\left((1,0)\left(f_{1}, f_{2}\right)\right)=(1,0) D\left(f_{1}, f_{2}\right) \in V_{1},
$$


análogamente:

$$
D\left(0, f_{2}\right)=D\left((0,1)\left(f_{1}, f_{2}\right)\right)=(0,1) D\left(f_{1}, f_{2}\right) \in V_{2},
$$

luego:

$$
\operatorname{Hom}_{V_{1} \times V_{2}}\left(\Omega_{V_{1} / k} \oplus \Omega_{V_{2} / k}, V_{1} \times V_{2}\right) \simeq \operatorname{Hom}_{V_{1}}\left(\Omega_{V_{1} / k}, V_{1}\right) \oplus \operatorname{Hom}_{V_{2}}\left(\Omega_{V_{2} / k}, V_{2}\right)
$$

y se concluye:

$$
\operatorname{Der}_{k}(V, V) \simeq \operatorname{Der}_{k}\left(V_{1}, V_{1}\right) \oplus \operatorname{Der}_{k}\left(V_{2}, V_{2}\right)
$$

Proposición 2.3. Se tiene el siguiente isomorfismo de álgebras de Lie:

$$
\text { Lie } \mathrm{G}_{k((z))}^{V} \stackrel{\sim}{\longrightarrow} \text { Lie } \mathrm{G}
$$

Demostración. La proposición 1.14 nos da una sucesión exacta:

$$
0 \rightarrow \operatorname{Aut}_{k((z))-a l g} V \rightarrow \mathrm{G}_{k((z))}^{V} \rightarrow \mathrm{G} \rightarrow 0
$$

y como el álgebra de Lie de $\operatorname{Aut}_{k((z))-a l g} V$ es trivial, pues es un grupo discreto, se tiene el isomorfismo:

$$
\text { Lie } \mathrm{G}_{k((z))}^{V} \stackrel{\sim}{\rightarrow} \text { Lie G }
$$

Definición 2.4. Sea $\pi: k((z)) \hookrightarrow V$ la estructura de $k((z))$-álgebra de V. Se definen las derivaciones compatibles con $\pi$, $\operatorname{Der}_{k}(V, V)^{\pi}$, como las derivaciones $D \in \operatorname{Der}_{k}(V, V)$ para las que existe $D^{\prime} \in \operatorname{Der}_{k}(k((z)), k((z)))$ verificando:

$$
D \circ \pi=\pi \circ D^{\prime} \text {. }
$$

Sea $\operatorname{Tr}: V \rightarrow k((z))$ el morfismo traza. De modo análogo se define $\operatorname{Der}_{k}(V, V)^{\operatorname{Tr}}$.

Corolario 2.5. Se tienen los siguientes isomorfismos de álgebras de Lie:

$$
\text { Lie } \left.\mathrm{G}_{k((z))}^{V} \stackrel{\mathrm{Der}}{\sim} V, V\right)^{\pi} \simeq \operatorname{Der}(V, V)^{\operatorname{Tr}} \simeq \text { Lie G }
$$

Demostración. El primer isomorfismo se sigue de la Proposición 2.1 y la definición de $\mathrm{G}_{k((z))}^{V}$ (Definición 1.8p; el segundo se deduce usando además la Proposición 1.12, el último es consecuencia de la proposición anterior.

\subsection{Operadores diferenciales como álgebras de Lie.}

En esta sección veremos que ciertos operadores diferenciales tienen una estructura natural de álgebra de Lie y seremos además capaces de integrar estas álgebras, concluyendo que son las álgebras de Lie de los grupos de automorfismos semilineales del capítulo anterior. Utilizaremos frecuentemente las definiciones y propiedades de los operadores diferenciales, resumidas en el Apéndice C. 


\subsubsection{Operadores diferenciales y $\operatorname{Lie}\left(\operatorname{SGl}_{k((z))}(V)\right)$.}

De modo análogo al apéndice C, pueden definirse los operadores diferenciales de orden $\leq 1$ de $V$ en $V$ sobre $k((z)), \mathcal{D i f f}_{k((z)) / k}^{1}(V, V)$. Recordemos además que se tiene una sucesión exacta:

$$
0 \rightarrow \operatorname{End}_{k((z))} V \rightarrow \operatorname{Diff}_{k((z)) / k}^{1}(V, V) \stackrel{\sigma}{\rightarrow} \operatorname{Der}_{k}(k((z)), k((z))) \otimes_{k((z))} \operatorname{End}_{k((z))} V \rightarrow 0
$$

siendo $\sigma$ el morfismo símbolo.

Denotemos $\mathcal{D}_{k((z)) / k}^{1}(V, V)$ a los operadores en $\mathcal{D i f f}_{k((z)) / k}^{1}(V, V)$ con símbolo escalar, es decir, aquellos cuya derivación asociada vía el morfismo símbolo valora en:

$$
\operatorname{Der}_{k}(k((z))) \otimes_{k((z))} k((z)) \hookrightarrow \operatorname{Der}_{k}(k((z))) \otimes_{k((z))} \operatorname{End}_{k((z))} V
$$

donde $k((z)) \hookrightarrow \operatorname{End}_{k((z))} V$ es el morfismo canónico. Equivalentemente, los operadores $P \in \mathcal{D}_{k((z)) / k}^{1}(V, V)$ vienen caracterizados por la fórmula:

$$
P(z v)=D(z) v
$$

donde $D=\sigma(P)$. De la sucesión 2.2.1 se deduce:

$$
0 \rightarrow \operatorname{End}_{k((z))} V \rightarrow \mathcal{D}_{k((z)) / k}^{1}(V, V) \stackrel{\sigma}{\rightarrow} \operatorname{Der}_{k}(k((z))) \rightarrow 0 .
$$

Los operadores con símbolo escalar, $\mathcal{D}_{k((z)) / k}^{1}(V, V)$, poseen además una estructura natural de subálgebra de Lie dentro de $\operatorname{End}_{k} V$, pues su paréntesis de Lie vuelve a ser un elemento de $\mathcal{D}_{k((z)) / k}^{1}(V, V)$ :

$$
\begin{aligned}
{\left[P_{1}, P_{2}\right](z v) } & =P_{1} P_{2}(z v)-P_{2} P_{1}(z v)=P_{1}\left(z P_{2}(v)+D_{2}(z) v\right)-P_{2}\left(z P_{1}(v)+D_{1}(z) v\right)= \\
& =z P_{1} P_{2}(v)+D_{1}(z) P_{2}(v)+D_{2}(z) P_{1}(v)+D_{1} D_{2}(z) v- \\
& -z P_{2} P_{1}(v)-D_{2}(z) P_{1}(v)-D_{1}(z) P_{2}(v)-D_{2} D_{1}(z) v= \\
& =z\left[P_{1}, P_{2}\right](v)+\left[D_{1}, D_{2}\right](z) v
\end{aligned}
$$

siendo $D_{1}=\sigma\left(P_{1}\right), D_{2}=\sigma\left(P_{2}\right)$ las derivaciones asociadas, vía el morfismo símbolo, a los operadores diferenciales $P_{1}$ y $P_{2}$ y por tanto $\sigma\left(\left[P_{1}, P_{2}\right]\right)=\left[D_{1}, D_{2}\right]$.

Observación 2.6. No es cierto sin embargo que $\operatorname{Diff}_{k((z)) / k}^{1}(V, V)$ tenga estructura de álgebra de Lie, nótese que en el cálculo anterior se utiliza fuertemente que la derivación asociada a un operador diferencial con símbolo escalar es una homotecia de razón un elemento de $k((z))$.

Proposición 2.7. Se tiene un isomorfismo canónico de álgebras de Lie:

$$
\operatorname{Lie}\left(\operatorname{SGl}_{k((z))}(V)\right) \simeq \mathcal{D}_{k((z)) / k}^{1}(V, V)
$$


Demostración. Sea $k[\epsilon]$ el anillo de los números duales. Por definición:

$$
\text { Lie }\left(\operatorname{SGl}_{k((z))}(V)\right):=\operatorname{SGl}_{k((z))}(V)(k[\epsilon]) \times \operatorname{SGl}_{k((z))}(V)(k)\{\operatorname{Id}\}
$$

luego sus elementos corresponden a automorfismos:

$$
\gamma: V \oplus \epsilon V \simeq V \oplus \epsilon V
$$

$k[\epsilon]$-lineales tales que $\gamma_{\mid \epsilon=0}=$ Id y para los que existe un automorfismo:

$$
g: k((z)) \oplus \epsilon k((z)) \simeq k((z)) \oplus \epsilon k((z))
$$

de $k[\epsilon]$-álgebras tal que $g_{\mid \epsilon=0}=1$ y $\gamma(z v)=g(z) \gamma(v)$.

Como $\gamma$ es un automorfismo $k[\epsilon]$-lineal podemos escribir:

$$
\gamma=\operatorname{Id}+\epsilon \gamma_{0}
$$

donde $\gamma_{0} \in \operatorname{End}_{k} V$. Por ser $g$ un automorfismo de $k[\epsilon]$-álgebras, se tiene que:

$$
g=1+\epsilon g_{0}
$$

con $g_{0} \in \operatorname{Der}_{k}(k((z)))$ y la condición:

$$
\left(\operatorname{Id}+\epsilon \gamma_{0}\right)(z v)=\left(1+\epsilon g_{0}\right)(z)\left(\operatorname{Id}+\epsilon \gamma_{0}\right)(v)
$$

nos dice que:

$$
\gamma_{0}(z v)=z \gamma_{0}(v)+g_{0}(z) v
$$

es decir, $\gamma_{0} \in \mathcal{D}_{k((z)) / k}^{1}(V, V)$ y su derivación asociada vía el morfismo símbolo es $g_{0}$. Tenemos entonces un isomorfismo de $k$-espacios vectoriales:

$$
\begin{aligned}
\operatorname{Lie}\left(\operatorname{SGl}_{k((z))}(V)\right) & \simeq \mathcal{D}_{k((z)) / k}^{1}(V, V) \\
\operatorname{Id}+\epsilon \gamma_{0} & \mapsto \gamma_{0} .
\end{aligned}
$$

Ver que los paréntesis de Lie coinciden es inmediato.

Observación 2.8. Tomando álgebras de Lie en la sucesión:

$$
0 \rightarrow \mathrm{Gl}_{k((z))}(V) \rightarrow \mathrm{SGl}_{k((z))}(V) \rightarrow \mathrm{G} \rightarrow 0
$$

de la Proposición 1.21 se deduce la sucesión 2.2.2):

$$
0 \rightarrow \operatorname{End}_{k((z))} V \rightarrow \mathcal{D}_{k((z)) / k}^{1}(V, V) \stackrel{\sigma}{\rightarrow} \operatorname{Der}_{k}(k((z)), k((z))) \rightarrow 0 .
$$




\subsubsection{Operadores diferenciales y Lie $\left(\mathrm{SHeis}_{V}\right)$.}

Denotemos $\operatorname{Diff}_{V / k}^{1}(V, V)$ a los operadores diferenciales de orden $\leq 1$ de $V$ en $V$ sobre $V$ (es decir, $V$ como $V$-módulo). Se tiene una sucesión exacta:

$$
0 \rightarrow V \rightarrow \mathcal{D i f f}_{V / k}^{1}(V, V) \stackrel{\sigma}{\rightarrow} \operatorname{Der}_{k}(V, V) \rightarrow 0
$$

siendo $\sigma$ el morfismo símbolo.

Observación 2.9. El morfismo $P \mapsto P(1)$ es un retracto de:

$$
V \rightarrow \operatorname{Diff}_{V / k}^{1}(V, V)
$$

y por lo tanto la sucesión 2.2.3 rompe. Podemos entonces escribir todo operador diferencial $P \in \operatorname{Diff}_{V / k}^{1}(V, V)$ como:

$$
P=v+D, \quad v \in V, D \in \operatorname{Der}_{k}(V, V)
$$

Observación 2.10. Observemos que en este caso $\operatorname{Difff}_{V / k}^{1}(V, V) \stackrel{\sim}{\rightarrow} \mathcal{D}_{V / k}^{1}(V, V)$ y por lo tanto, como en la sección anterior, puede verse que tiene una estructura natural de álgebra de Lie.

Proposición 2.11. Se tiene un isomorfismo canónico de álgebras de Lie:

$$
\operatorname{Lie}\left(\text { SHeis }_{V}\right) \simeq \mathcal{D}_{V / k}^{1}(V, V)
$$

Demostración. Recordemos (véase la Definición 1.23) que si $\gamma \in \mathrm{SHeis}_{V}$, entonces:

$$
\gamma(v)=w \cdot g(v) \quad w \in V^{*}, \quad g \in \mathrm{G}_{V} .
$$

En virtud de la proposición 2.7 se tiene un isomorfismo de $k$-espacios vectoriales:

$$
\begin{aligned}
\operatorname{Lie}\left(\operatorname{SHeis}_{V}\right) & \simeq \mathcal{D}_{V / k}^{1}(V, V) \\
\operatorname{Id}+\epsilon \gamma_{0} & \mapsto \gamma_{0}
\end{aligned}
$$

donde $\gamma_{0}(v)=w_{0} v+g_{0}(v), g_{0} \in \operatorname{Der}_{k}(V, V) \simeq$ Lie $_{V}$ es la derivación asociada vía el morfismo símbolo y $w_{0} \in V \simeq$ Lie $V^{*}$ (véase la ecuación (2.0.3) para este isomorfismo). Ver que los paréntesis de Lie coinciden es inmediato en virtud de la sucesión 2.2.3.

Observación 2.12. Tomando álgebras de Lie en la sucesión:

$$
0 \rightarrow \text { Heis }_{V} \rightarrow \text { SHeis }_{V} \rightarrow \mathrm{G}_{V} \rightarrow 0
$$

de la Proposición 1.24 se deduce la sucesión 2.2.3. 


\subsubsection{Operadores diferenciales y Lie $\left(\operatorname{SHeis}_{k((z))}^{V}\right)$.}

Definimos ahora una nueva versión de los operadores diferenciales de orden $\leq 1$ de $V$ en $V$ sobre $V, \operatorname{Diff}_{V / k}^{1}(V, V)$, haciendo uso de la estructura $\pi$ de $k((z))$-álgebra de $V$.

Definición 2.13. Llamaremos operadores diferenciales de orden $\leq 1$ de $V$ en $V$ sobre $V$ compatibles con $\pi, \mathcal{D i f f}_{V / k}^{1}(V, V)^{\pi}$, a aquellos cuyo morfismo símbolo valora en $\operatorname{Der}_{k}(V, V)^{\pi}$ (Definición 2.47.De este modo, se tiene un diagrama:

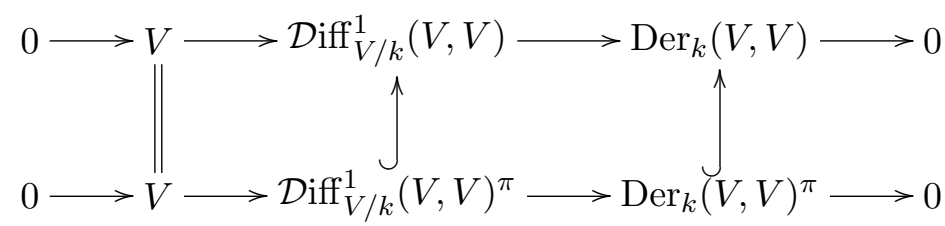

donde el segundo cuadrado es cartesiano.

Proposición 2.14. Se tiene un isomorfismo canónico de álgebras de Lie:

$$
\operatorname{Lie}\left(\operatorname{SHeis}_{k((z))}^{V}\right) \simeq \mathcal{D}_{V / k}^{1}(V, V)^{\pi}
$$

Demostración. Se sigue de la Proposición 2.11 y el Corolario 2.5

Observación 2.15. Tomando álgebras de Lie en la sucesión:

$$
0 \rightarrow \operatorname{Heis}_{V} \rightarrow \operatorname{SHeis}_{k((z))}^{V} \rightarrow \mathrm{G}_{k((z))}^{V} \rightarrow 0
$$

de la Proposición 1.28 se deduce la sucesión:

$$
0 \rightarrow V \rightarrow \mathcal{D i f f}_{V / k}^{1}(V, V)^{\pi} \rightarrow \operatorname{Der}_{k}(V, V)^{\pi} \rightarrow 0
$$

Observación 2.16. Dado que $\operatorname{Der}_{k}(V, V)^{\pi} \simeq \operatorname{Der}_{k}(V, V)^{\operatorname{Tr}}$ (Corolario 2.5), se sigue que:

$$
\operatorname{Diff}_{V / k}^{1}(V, V)^{\operatorname{Tr}} \simeq \operatorname{Diff}_{V / k}^{1}(V, V)^{\pi}
$$

(donde se define $\mathcal{D i f f}_{V / k}^{1}(V, V)^{\operatorname{Tr}}$ como lo operadores cuyo morfismo símbolo valora en $\left.\operatorname{Der}_{k}(V, V)^{\operatorname{Tr}}\right)$. 


\section{Parte II}

\section{Espacios de Moduli}





\section{Capítulo 3}

\section{Moduli de curvas y fibrados.}

Estudiamos en este capítulo el moduli de curvas y fibrados con trivializaciones formales (permitiendo variar ambos datos). Abordaremos este problema de un modo ligeramente distinto al dado por Mulase en [28], puesto que nosotros no permitiremos una estructura local de revestimiento cíclico alrededor de un punto fijo de la curva. Caracterizamos á la Mulase la imagen del morfismo de Krichever, lo que nos permite demostrar que este espacio de moduli tiene estructura de subesquema dentro de una Grassmanniana infinita. Incluimos un último apartado en el que se generalizan los resultados al caso en el que la curva pueda ser singular. Lo que hace posible definir el morfismo de Krichever en el caso singular, es el hecho de imponer que el punto marcado sea un punto no singular.

\subsection{Morfismo de Krichever.}

Trabajaremos siempre sobre un cuerpo $k$ algebraicamente cerrado de característica nula. El objetivo es definir el morfismo de Krichever para un espacio de moduli cuyos puntos racionales sean datos $(X, x, z, E, \phi)$, donde $X$ es una curva lisa sobre $k$ con un punto marcado $x \in X, z$ es un parámetro formal en $x$ o, equivalentemente, un isomorfismo:

$$
z: \widehat{\mathcal{O}}_{X, x} \simeq k[[z]],
$$

$E$ es un fibrado vectorial de rango $n$ en $X$ y $\phi$ es un isomorfismo de $\widehat{\mathcal{O}}_{X, x}$-módulos:

$$
\phi: \widehat{E}_{x} \simeq \widehat{\mathcal{O}}_{X, x}^{n} .
$$

Definamos primero el espacio de moduli de curvas punteadas con trivialización formal.

Definición 3.1. ([33, Def.6.2]) Se define el functor $\mathcal{M}^{\infty}$ sobre la categoría de $k$-esquemas como el hacificado de:

$$
S \rightsquigarrow\{\text { familias }(X, D, z) \text { sobre } S\} / \sim
$$

donde: 
1. $\pi: X \rightarrow S$ es un morfismo propio y plano cuyas fibras geométricas son curvas lisas e íntegras.

2. $\sigma: S \rightarrow X$ es una sección de $\pi$ tal que su divisor de Cartier asociado, $D=\sigma(S)$, es liso, de grado relativo uno y plano sobre $S$. (Entenderemos que $D \subset X$ es liso sobre $S$ cuando para cada punto cerrado $x \in D$ exista un entorno $U$ de $x$ en $X$ de modo que el morfismo $U \rightarrow S$ es liso).

3. $z$ es una trivialización formal de $X$ a lo largo de $D$; esto es, una familia de morfismos epiyectivos de anillos:

$$
\mathcal{O}_{X} \rightarrow \sigma_{*}\left(\mathcal{O}_{S}[z] / z^{n} \mathcal{O}_{S}[z]\right) \quad n \in \mathbb{N}
$$

compatibles con las proyecciones canónicas:

$$
\mathcal{O}_{S}[z] / z^{n} \mathcal{O}_{S}[z] \rightarrow \mathcal{O}_{S}[z] / z^{n^{\prime}} \mathcal{O}_{S}[z] \quad n \geq n^{\prime}
$$

y tal que para $n=1$ coincida con $\sigma$.

4. Diremos que dos familias $(X, D, z)$ y $\left(X^{\prime}, D^{\prime}, z^{\prime}\right)$ son equivalentes cuando exista un isomorfismo $f: X \rightarrow X^{\prime}$ sobre $S$ tal que:

- $f^{*} D^{\prime}=D$.

- $f$ es compatible con las trivializaciones formales $z$ y $z^{\prime}$; esto es, el siguiente diagrama ha de ser conmutativo:

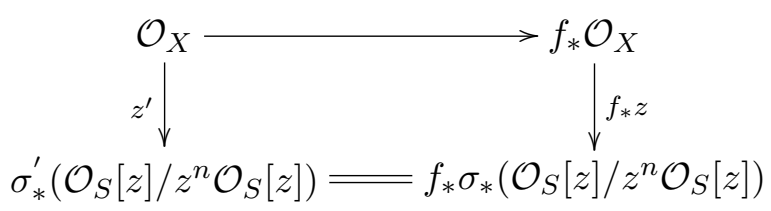

Sus puntos racionales son ternas formadas por una curva lisa $X$ con un punto marcado $x \in X$ y un parámetro formal $z$ en el punto.

Definición 3.2. Se define el functor $\mathcal{U}^{\infty}$ sobre la categoría de $k$-esquemas como el hacificado de:

$$
S \rightsquigarrow\{\text { familias }(X, D, z, E, \phi) \text { sobre } S\} / \sim
$$

donde:

1. $(X, D, z) \in \mathcal{M}^{\infty}(S)$.

2. $E$ es una familia de fibrados vectoriales de rango $n$ en $X$ plana sobre $S$. 
3. $\phi$ es una trivialización formal de $E$ a lo largo de $D$; esto es, una familia de morfismos epiyectivos de $\mathcal{O}_{X}$-módulos:

$$
E \rightarrow\left(\mathcal{O}_{X} / \mathcal{O}_{X}(-m D)\right)^{n} \quad m \in \mathbb{N}
$$

compatibles con los morfismos naturales:

$$
\mathcal{O}_{X} / \mathcal{O}_{X}(-m D) \rightarrow \mathcal{O}_{X} / \mathcal{O}_{X}(-(m-1) D)
$$

4. Fijado un $k$-esquema $S$, diremos que dos familias $(X, D, z, E, \phi)$ y $\left(X^{\prime}, D^{\prime}, z^{\prime}, E^{\prime}, \phi^{\prime}\right)$ son equivalentes si existe un isomorfismo $f: X \simeq X^{\prime}$ sobre $S$ tal que $(X, D, z) \sim$ $\left(X^{\prime}, D^{\prime}, z^{\prime}\right)$, y si existe además un isomorfismo $g: f^{*} E^{\prime} \simeq E$ compatible con las trivializaciones formales $\phi$ y $\phi^{\prime}$; esto es, el siguiente diagrama ha de ser conmutativo:

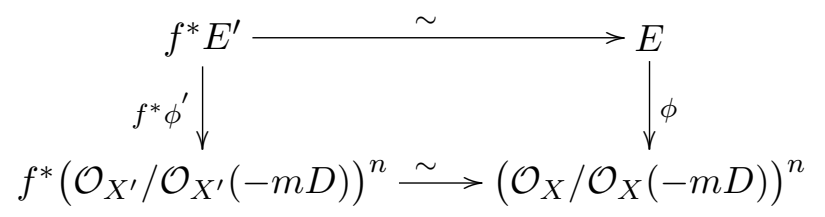

donde el isomorfismo de la segunda fila se sigue de la identificación $f^{*} D^{\prime}=D$.

Se tiene que la trivialización formal $\phi$ induce una epiyección:

$$
E \longrightarrow \underset{m}{\lim } \mathcal{O}_{X} / \mathcal{O}_{X}(-m D)=\widehat{\mathcal{O}}_{X, D}^{n}, \longrightarrow 0
$$

que factoriza por la completación de $E$ a lo largo de $D$ :

$$
\widehat{E}_{D} \rightarrow \widehat{\mathcal{O}}_{X, D}^{n}
$$

y es de hecho un isomorfismo, pues ambos son $\widehat{\mathcal{O}}_{X, D}$-módulos localmente libres de rango $n$ $\mathrm{y} \lim _{\longleftarrow} E(-m D)=0$.

Un punto racional de $\mathcal{U}^{\infty}$ corresponde a una quintupla de datos $(X, x, z, E, \phi)$, donde $X$ es una curva lisa con un punto marcado $x \in X, z$ es un parámetro formal en $x \mathrm{o}$, equivalentemente, un isomorfismo:

$$
z: \widehat{\mathcal{O}}_{X, x} \simeq k[[z]],
$$

$E$ es un fibrado vectorial de rango $n$ en $X$ y $\phi$ es una trivialización formal en $x$, es decir, un isomorfismo de $\widehat{\mathcal{O}}_{X, x}$-módulos:

$$
\phi: \widehat{E}_{x} \simeq \widehat{\mathcal{O}}_{X, x}^{n} .
$$

Definición 3.3. Se define el morfismo de Krichever para el functor $\mathcal{U}^{\infty}$ como el morfismo de functores:

$$
\begin{aligned}
K r: \mathcal{U}^{\infty}(S) & \rightarrow \operatorname{Gr}\left(k((z))^{n}\right)(S) \\
(X, D, z, E, \phi) & \mapsto(z \circ \phi) \underset{m^{\prime}}{\lim } \pi_{*} E\left(m^{\prime} D\right)
\end{aligned}
$$

donde $\operatorname{Gr}\left(k((z))^{n}\right)$ es la Grassmanniana infinita algebraica construida en el apéndice $\mathrm{A}$ 
Veamos que en efecto este límite define un punto de $\operatorname{Gr}\left(k((z))^{n}\right)$. Consideremos el diagrama:

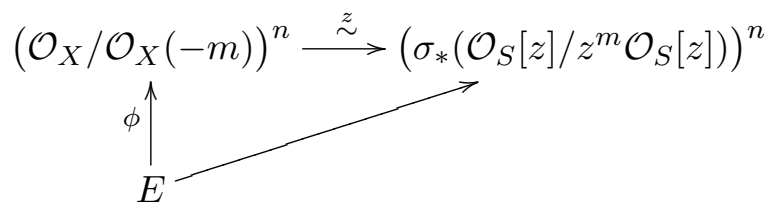

Puede demostrarse que la siguiente sucesión es exacta:

$$
0 \rightarrow E(-m D) \rightarrow E \rightarrow\left(\sigma_{*}\left(\mathcal{O}_{S}[z] / z^{m} \mathcal{O}_{S}[z]\right)\right)^{n} \rightarrow 0,
$$

y en consecuencia, se tiene también la sucesión exacta:

$$
0 \rightarrow E(-m D) \rightarrow E\left(m^{\prime} D\right) \rightarrow\left(\sigma_{*}\left(z^{-m^{\prime}} \mathcal{O}_{S}[z] / z^{m} \mathcal{O}_{S}[z]\right)\right)^{n} \rightarrow 0 \quad m, m^{\prime} \geq 0
$$

Siguiendo los pasos de la construcción del morfismo de Krichever de [2] se concluye que:

$$
(z \circ \phi) \underset{m^{\prime}}{\lim _{*}} \pi_{*} E\left(m^{\prime} D\right) \in \operatorname{Gr}\left(k((z))^{n}\right)(S)
$$

Observación 3.4. Para ilustrar el argumento anterior, detallaremos en el caso de puntos racionales, $S=$ Spec $k$, cómo ver que el morfismo de Krichever:

$$
(X, x, z, E, \phi) \mapsto(z \circ \phi) \mathrm{H}^{0}(X-x, E)
$$

define un punto de $\operatorname{Gr}\left(k((z))^{n}\right)$. Denotemos $W=(z \circ \phi) \mathrm{H}^{0}(X-x, E)$ y veamos que $W$ es de hecho un punto de:

$$
\operatorname{Gr}\left(k((z))^{n}\right)(k)=\left\{\begin{array}{c}
W \subseteq k((z))^{n} \text { tales que } W \cap k[[z]]^{n} \text { y } k((z))^{n} / W+k[[z]]^{n} \\
\text { son } k \text {-espacios vectoriales de dimensión finita }
\end{array}\right\}
$$

Mandando una sección en $\mathrm{H}^{0}(X-x, E)$ a su germen en $x$ (usando $z \mathrm{y} \phi$ ) tenemos una inclusión:

$$
W=(z \circ \phi) \mathrm{H}^{0}(X-x, E) \hookrightarrow\left(\widehat{\mathcal{O}}_{X, x}\right)_{0}^{n} \simeq k((z))^{n}
$$

Consideremos la sucesión exacta de haces:

$$
0 \rightarrow E \rightarrow E(m x) \rightarrow\left(\mathcal{O}_{m x}(m x)\right)^{n} \rightarrow 0
$$

y tomemos cohomología:

$$
0 \rightarrow \mathrm{H}^{0}(X, E) \rightarrow \mathrm{H}^{0}(X, E(m x)) \rightarrow\left(\mathcal{O}_{m x}(m x)\right)^{n} \rightarrow \mathrm{H}^{1}(X, E) \rightarrow \mathrm{H}^{1}(X, E(m x)) \rightarrow 0
$$

donde el último 0 se deduce por $\operatorname{ser} \mathcal{O}_{m x}(m x)$ un haz flasgo (está concentrado en $x$ ). Sabemos además que para $m$ suficientemente grande es $\mathrm{H}^{1}(X, E(m x))=0$, luego tenemos:

$$
0 \rightarrow \mathrm{H}^{0}(X, E) \rightarrow \mathrm{H}^{0}(X, E(m x)) \rightarrow\left(\mathcal{O}_{m x}(m x)\right)^{n} \rightarrow \mathrm{H}^{1}(X, E) \rightarrow 0
$$


Como $\mathcal{O}_{m x}(m x)$ es el cociente entre la funciones meromorfas en $X$ con posible polo en $x$ de orden a lo más $m$ y las funciones regulares en $X$, se sigue que:

$$
\mathcal{O}_{m x}(m x)=\left\langle z^{-1}, \ldots, z^{-m}\right\rangle
$$

Tomando $\lim _{\longrightarrow}$ en la sucesión 3.1 .1 obtenemos:

$$
0 \rightarrow \mathrm{H}^{0}(X, E) \rightarrow W \rightarrow k((z))^{n} / k[[z]]^{n} \rightarrow \mathrm{H}^{1}(X, E) \rightarrow 0
$$

pues:

$$
\underset{m}{\lim } \mathcal{O}_{m x}(m x)=k((z)) / k[[z]] \quad \text { y } \quad \underset{m}{\lim } \mathrm{H}^{0}(X, E(m x))=\mathrm{H}^{0}(X-x, E)=W .
$$

En virtud de la finitud de la cohomología se concluye que:

$$
W=\mathrm{H}^{0}(X-x, E) \in \operatorname{Gr}\left(k((z))^{n}\right)(k) .
$$

Definimos ahora un morfismo de Krichever para $\mathcal{U}^{\infty}$ que valora en dos Grassmannianas, que nos será de utilidad a la hora de representar $\mathcal{U}^{\infty}$.

Definición 3.5. Se define el morfismo de Krichever para el functor $\mathcal{U}^{\infty}$ como el morfismo de functores:

$$
\begin{aligned}
K r_{2}: \mathcal{U}^{\infty}(S) & \rightarrow \operatorname{Gr}\left(k((z))^{n}\right)(S) \times \operatorname{Gr}(k((z)))(S) \\
(X, D, z, E, \phi) & \mapsto\left((z \circ \phi) \underset{\overrightarrow{m^{\prime}}}{\lim } \pi_{*} E\left(m^{\prime} D\right), z\left(\underset{m^{\prime}}{\lim } \pi_{*} \mathcal{O}_{X}\left(m^{\prime} D\right)\right)\right)
\end{aligned}
$$

Observación 3.6. Cuando $S=\operatorname{Spec} k$, el morfismo viene definido por:

$$
(X, x, z, E, \phi) \mapsto\left((z \circ \phi) \mathrm{H}^{0}(X-x, E), z \mathrm{H}^{0}\left(X-x, \mathcal{O}_{X}\right)\right)
$$

Observación 3.7. Sea $V$ un $k$-espacio vectorial, $V^{+}$un subespacio destacado de $V$ y $W \in$ $\operatorname{Gr}(V)$. Se define la función índice:

$$
i: \operatorname{Gr}(V) \rightarrow \mathbb{Z}
$$

por $i(W)=\operatorname{dim}_{k}\left(W \cap V^{+}\right)-\operatorname{dim}_{k}\left(V / W+V^{+}\right)$. Tenemos entonces una descomposición de la Grassmanniana en componentes conexas (véase [3]):

$$
\operatorname{Gr}(V)=\coprod_{m \in \mathbb{Z}} \operatorname{Gr}^{m}(V)
$$

$\operatorname{Si}(X, x, z) \in \mathcal{M}_{g}^{\infty}(k)$, es decir, si hemos fijado además el género $g$ de nuestra curva $X$, se tiene una descomposición en componentes conexas:

$$
\mathcal{M}^{\infty}=\coprod_{g} \mathcal{M}_{g}^{\infty}
$$


y la función índice equivale, vía Krichever, a la característica de Euler-Poincaré del haz estructural $\mathcal{O}_{X}$, luego el morfismo de Krichever $\mathcal{M}_{g}^{\infty}$ a la componente conexa $\operatorname{Gr}^{1-g}(k((z)))$.

Análogamente, si denotamos $\mathcal{U}_{g, d}^{\infty}$ al moduli en el que las curvas tiene género arimético $g$ y los fibrados tienen grado $d$ (el rango ya lo tenemos fijo), entonces los morfismos de Krichever se escriben:

$$
\begin{gathered}
\mathrm{Kr}: \mathcal{U}_{g, d}^{\infty} \rightarrow \operatorname{Gr}^{n(1-g)+d}\left(k((z))^{n}\right) \\
\mathrm{Kr}_{2}: \mathcal{U}_{g, d}^{\infty} \rightarrow \operatorname{Gr}^{n(1-g)+d}\left(k((z))^{n}\right) \times \mathrm{Gr}^{1-g}(k((z)))
\end{gathered}
$$

\subsection{Caracterización de la imagen.}

En esta sección nos proponemos demostrar que el morfismo de Krichever es inyectivo y caracterizar su imagen, es decir, dar condiciones para comprobar cuándo un punto de la Grassmanniana proviene de datos en $\mathcal{U}^{\infty}$. La idea es la siguiente, sea $W$ un punto racional de la Grassmanniana $\operatorname{Gr}\left(k((z))^{n}\right)$ y $A_{W} \subset k((z))$ el conjunto de $f \in k((z))$ tales que $f \cdot W \subseteq W$ (estabilizador de $W$ en $k((z))$ ). Por definición $W$ es $A_{W}$-módulo y $A_{W}$ es una sub- $k$-álgebra de $k((z))$. Daremos una construcción inversa del morfismo de Krichever, demostrando que con $A_{W}$ recuperamos una curva, un punto y un parámetro formal del mismo, y con $W$ construiremos un fibrado sobre dicha curva y una trivialización formal en el punto. Necesitaremos primero dar una serie de resultados técnicos, que nos permitirán extrapolar esta idea al caso de puntos con valores en cualquier $k$-esquema, y por lo tanto, hacerla válida para familias de curvas y fibrados.

Sea $S$ un $k$-esquema.

Lema 3.8. Sea $A \hookrightarrow \mathcal{O}_{S}((z))$ un subhaz cuasicoherentes (como $\mathcal{O}_{S}$-módulo). Si $\mathcal{O}_{S}((z)) / A$ es plano sobre $S$, entonces $A$ es plano sobre $S$ y para cada punto $s \in S, A_{s} \subseteq \kappa(s)((z))$ (donde $A_{s}=A \otimes_{\mathcal{O}_{S}} \kappa(s)$ es el cambio de base de $A$ a $s$ ).

Demostración. Como $\mathcal{O}_{S}((z))$ es plano sobre $S$ y $\mathcal{O}_{S}((z)) / A$ es plano por hipótesis, resulta que $A$ es plano sobre $S$. En particular, $\operatorname{Tor}_{1}^{\mathcal{O}_{S}}\left(\mathcal{O}_{S}((z)) / A, \kappa(s)\right)=0$, luego se tiene una sucesión exacta:

$$
0 \rightarrow \operatorname{Tor}_{1}^{\mathcal{O}_{S}}\left(\mathcal{O}_{S}((z)) / A, \kappa(s)\right)=0 \rightarrow A_{s} \rightarrow \kappa(s)((z)) \rightarrow \kappa(s)((z)) / A_{s} \rightarrow 0
$$

y por tanto $A_{s} \subseteq \kappa(s)((z))$.

Definición 3.9. Sea $W \in \operatorname{Gr}\left(k((z))^{n}\right)(S)$ un punto de la Grassmaniana con valores en $S$. Se dice que una sub- $\mathcal{O}_{S}$-álgebra $A$ de $\mathcal{O}_{S}((z))$ estabiliza $W$ si verifica:

1. $\mathcal{O}_{S}((z)) / A$ es plano sobre $S$.

2. $A \cdot W \subseteq W$.

Se llama estabilizador de $W$ a la máxima subálgebra de $\mathcal{O}_{S}((z)), A_{W}$, que estabiliza $W$. 
El estabilizador existe por el lema de Zorn, pues la familia de sub- $\mathcal{O}_{S}$-álgebras que estabiliza $W$ no es vacía (contiene a $\mathcal{O}_{S}$ ) y una unión de subálgebras que estabilizan $W$ también estabiliza $W$. Además $\mathcal{O}_{S} \subseteq U$ para toda subálgebra $U$ de $\mathcal{O}_{S}((z))$ que estabiliza $W$.

Si $S=$ Spec $k$, con $k$ cuerpo, la condición de platitud es automática y el estabilizador es simplemente:

$$
A_{W}=\{f \in k((z)) \mid f \cdot W \subseteq W\} .
$$

Observación 3.10. Si $A$ estabiliza $W$, entonces para todo cambio de base $T \rightarrow S$ y en virtud de la platitud de $\mathcal{O}_{S}((z)) / A$, se tiene que $A_{T} \subseteq \mathcal{O}_{T}((z))$. Además $A_{T}$ estabiliza a $W_{T}$ y en particular $\left(A_{W}\right)_{T}$ estabiliza $W_{T}$, luego $\left(A_{W}\right)_{T} \subseteq A_{W_{T}}$. No obstante, esta inclusión no tiene porqué ser una igualdad.

Observemos que esta última afirmación nos dice que en realidad el estabilizador $A_{W}$ no es una familia buena, en el sentido de que cambiando de base $T \rightarrow S,\left(A_{W}\right)_{T}$ podría dejar de ser la máxima álgebra que estabiliza a $W_{T}$. Sin embargo, veremos que en los casos en que $A$ y $W$ vienen de datos geométricos (veáse el Lema 3.14 y la Observación 3.25), siempre se verifica que $\left(A_{W}\right)_{T}=A_{W_{T}}$. Resulta así que el estabilizador representa una familia de estabilizadores. Finalmente, si $A \subseteq \tilde{A}$ estabilizan a $W$, se tiene también que $A_{T} \subseteq \tilde{A}_{T}$.

En lo que sigue escribiremos $V=k((z))^{n}$.

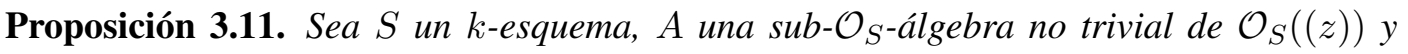
$W \in \operatorname{Gr}(V)(S)$ un A-módulo. Entonces:

$$
\mathrm{rk}_{A} W=n \Longleftrightarrow A \in \operatorname{Gr}(k((z)))(S)
$$

Demostración. Recordemos que se define el rango de $W$ sobre $A$ por:

$$
\mathrm{rk}_{A} W=n \cdot d
$$

siendo $d$ el grado de la extensión:

$$
(\widehat{A})_{0} \hookrightarrow \mathcal{O}_{S}((z)) .
$$

Veamos en primer lugar que como $W \in \operatorname{Gr}(V)(S)$ es $A$-módulo entonces $A \cap \mathcal{O}_{S}[[z]]=$ $\mathcal{O}_{S}$. En efecto, sea $V^{+}=k[[z]]^{n}$ y consideremos las filtraciones:

$$
\begin{gathered}
A^{(i)}:=A \cap z^{-i} \mathcal{O}_{S}[[z]] \\
W^{(i)}:=W \cap z^{-i} V_{S}^{+},
\end{gathered}
$$

por ser $W$ un punto de $\operatorname{Gr}(V)(S)$ se tiene que $W^{(0)}$ es un haz de $\mathcal{O}_{S}$-módulos localmente libre de tipo finito, luego existe $M \in \mathbb{Z}$ tal que:

$$
W^{(M)} \subset W^{(0)}, \quad W^{(M)} \neq\{0\}, \quad W^{(M-1)}=\{0\}
$$

Como $W$ es por hipótesis $A$-módulo se sigue:

$$
A^{(-1)} \cdot W^{(M)} \subseteq W^{(M-1)}=\{0\} \Longrightarrow A^{(-1)}=\{0\}
$$




$$
A^{(0)} \cdot W^{(M)} \subseteq W^{(M)} \Longrightarrow A^{(0)}=\mathcal{O}_{S}
$$

Luego $A^{(0)}:=A \cap \mathcal{O}_{S}[[z]]=\mathcal{O}_{S}$.

Supongamos que $\operatorname{rk}_{A} W=n$ y que $A \notin \operatorname{Gr}(k((z)))(S)$. Como $A \neq \mathcal{O}_{S}$ y $A \cap$ $\mathcal{O}_{S}[[z]]=\mathcal{O}_{S}$ se sigue que:

$$
A=\left\langle 1, z^{s_{1}}+O\left(z^{s_{1}+1}\right), z^{s_{2}}+O\left(z^{s_{2}+1}\right), z^{s_{3}}+O\left(z^{s_{3}+1}\right), \ldots\right\rangle \quad s_{i}<0 .
$$

Por ser $A$ una sub- $\mathcal{O}_{S}$-álgebra de $\mathcal{O}_{S}((z))$ existe un $m$ tal que:

$$
z^{s_{j}}+O\left(z^{s_{j}+1}\right) \in\left(z^{s_{1}}+O\left(z^{s_{1}+1}\right), \ldots, z^{s_{m}}+O\left(z^{s_{m}+1}\right)\right) \quad \forall j>>0
$$

es decir, basta un número finito:

$$
\left\{z^{s_{1}}+O\left(z^{s_{1}+1}\right), \ldots, z^{s_{m}}+O\left(z^{s_{m}+1}\right)\right\}
$$

para generar $A$.

Si el máximo común divisor de $s_{1}, \ldots, s_{m}$ es uno:

$$
\operatorname{MCD}\left(s_{1}, \ldots, s_{m}\right)=1
$$

entonces es fácil ver que para $k>>0$ se tiene que $s_{k+1}=s_{k}+1$ y por lo tanto $A \in$ $\operatorname{Gr}(k((z)))(S)$, que no es nuestro caso, luego podemos suponer que:

$$
\operatorname{MCD}\left(s_{1}, \ldots, s_{m}\right)=r \neq 1 .
$$

Se tiene así que $s_{j}=r \bar{s}_{j}$ para todo $j=1, \ldots, m, \mathrm{y} \operatorname{MCD}\left(\bar{s}_{1}, \ldots, \bar{s}_{m}\right)=1$. Podemos entonces escribir:

$$
A=\left\langle 1, z^{r \bar{s}_{1}}+O\left(z^{r \bar{s}_{1}+1}\right), z^{r \bar{s}_{2}}+O\left(z^{r \bar{s}_{2}+1}\right), z^{r \bar{s}_{3}}+O\left(z^{r \bar{s}_{3}+1}\right), \ldots\right\rangle .
$$

Se sigue entonces que el grado de la extensión:

$$
(\widehat{A})_{0} \hookrightarrow \mathcal{O}_{S}((z))
$$

es al menos $r$, y por lo tanto es estrictamente mayor que uno, con lo que llegamos a contradicción con que el rango de $W$ sea $n$. En consecuencia $A \in \operatorname{Gr}(k((z)))(S)$.

El recíproco es análogo.

Corolario 3.12. Sea $S$ un k-esquema y $W \in \operatorname{Gr}(V)(S)$ tal que $A_{W} \neq \mathcal{O}_{S}$. Entonces:

$$
\operatorname{rk}_{A_{W}} W=n \Longleftrightarrow A_{W} \in \operatorname{Gr}(k((z)))(S)
$$

Lema 3.13. Sean $A$ y $\tilde{A}$ dos puntos de $\operatorname{Gr}(k((z)))(S)$ tales que $A \subseteq \tilde{A}$ y para cada punto

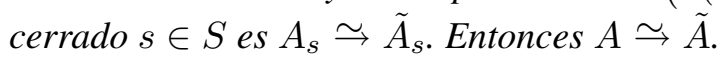


Demostración. Consideremos la sucesión exacta:

$$
0 \rightarrow A \rightarrow \tilde{A} \rightarrow \tilde{A} / A \rightarrow 0
$$

Sabemos que al tensorializar por $\kappa(s)(s=\operatorname{Spec} \kappa(s) \in S$ punto cerrado) se tiene que $A_{s}=\tilde{A}_{s}$, luego $(\tilde{A} / A)_{s}=0$. Veamos entonces que $\tilde{A} / A$ es de tipo finito y por Nakayama concluiremos que $\tilde{A} / A=0$ (esto es, $A=\tilde{A}$ ). Para lo cual basta con que sea localmente de tipo finito, pues es haz. En efecto, como ambos son puntos de $\operatorname{Gr}(k((z)))(S)$, para cada punto $s \in S$ existe un entorno abierto $U$ de $s$ en $S$ y subespacios $C, D$ en la topología $\tau$ de conmensurables (que podemos elegir de modo que $C \subset D$ ) tales que:

$$
\begin{aligned}
& A_{U} \oplus \widehat{C_{U}} \simeq \mathcal{O}_{U}((z)) \\
& \tilde{A}_{U} \oplus \widehat{D_{U}} \simeq \mathcal{O}_{U}((z))
\end{aligned}
$$

Luego $(\tilde{A} / A)_{U} \simeq(D / C)_{U}$ que es localmente libre de tipo finito pues $C, D \in \tau$.

Lema 3.14. Sean $(X, D, z, E, \phi) \in \mathcal{U}^{\infty}(k)$ un punto racional y $W \in \operatorname{Gr}(V)(k)$ el punto que define vía el morfismo de Krichever. Entonces:

$$
\mathrm{H}^{0}\left(X-x, \mathcal{O}_{X}\right)=A_{W} \in \operatorname{Gr}(k((z)))(k) .
$$

Demostración. En efecto, sea $A \in \operatorname{Gr}(k((z)))(k)$ el punto que define la terna $(X, x, z)$ y denotemos $\Sigma_{X}$ el cuerpo de funciones de $X$. Sabemos que:

$$
A=\mathrm{H}^{0}\left(X-x, \mathcal{O}_{X}\right)
$$

y como $E$ es $\mathcal{O}_{X}$ módulo, entonces $A \subseteq A_{W}$. Recordemos en qué sentido usamos las trivializaciones:

$$
W=\mathrm{H}^{0}(X-x, E) \stackrel{\phi}{\rightarrow} \Sigma_{X}^{n} \stackrel{z}{\rightarrow} k((z))^{n}
$$

Por definición:

$$
A_{W}:=\{f \in k((z)) \mid f \cdot W \subseteq W\}
$$

luego $f \in \Sigma_{X}$ (e induce una homotecia en $\Sigma_{X}^{n}$ ) y tiene un posible polo sólo en $x$. Como la curva $X$ es lisa entonces $f \in \mathrm{H}^{0}\left(X-x, \mathcal{O}_{X}\right)$, es decir $A=A_{W} \in \operatorname{Gr}(k((z)))(k)$.

Teorema 3.15. Sea $S$ un k-esquema, $(X, D, z, E, \phi) \in \mathcal{U}^{\infty}(S), W \in \operatorname{Gr}(V)(S)$ el punto que define vía el morfismo de Krichever y A el punto que define $(X, D, z)$ en $\operatorname{Gr}(k((z)))(S)$. Para cada morfismo de k-esquemas $T \rightarrow S$ es:

$$
A_{T}=\left(A_{W}\right)_{T}=A_{W_{T}} \in \operatorname{Gr}(k((z)))(T) .
$$

Demostración. Por la Observación 3.10 se tiene una cadena de inclusiones:

$$
A_{T} \subseteq\left(A_{W}\right)_{T} \subseteq A_{W_{T}}
$$

de puntos en $\operatorname{Gr}(k((z)))(T)$, que por lema anterior coinciden sobre los puntos cerrados $t=\operatorname{Spec} \kappa(t) \in T$ :

$$
A_{t}=\left(A_{W}\right)_{t}=A_{W_{t}} \in \operatorname{Gr}(k((z)))(\kappa(t)) .
$$

Se concluye usando el Lema 3.13 . 
Observación 3.16. Recordemos (Observación 3.10) que para cada morfismo de $k$-esquemas, $T \rightarrow S$, se tiene que $\left(A_{W}\right)_{T} \subseteq A_{W_{T}}$, y no siempre es una igualdad, lo que significa que $\left(A_{W}\right)_{T}$ dejaría de ser la máxima álgebra que estabilizara $W_{T}$. Lo que acabamos de ver es que si partimos de datos geométricos donde la curva es lisa, esta inclusión es siempre una igualdad y por tanto el estabilizador sigue siendo estabilizador para cualquier cambio de base. Y recíprocamente, si partimos de datos algebraicos $W$ y $A_{W}$ en $\operatorname{Gr}(V)$ y $\operatorname{Gr}(k((z)))$, respectivamente, y suponemos que $A_{W}$ es regular, entonces $A_{W}$ sigue siendo el estabilizador para cualquier cambio de base.

Definición 3.17. Sea $S$ un $k$-esquema y $A$ una $\mathcal{O}_{S}$-álgebra. Diremos que el anillo $A$ es relativamente regular sobre $S$, cuando para cada punto cerrado $s \in S$ se verifique que el cambio de base $A_{s}$ de $A$ a $s \in S$ sea un anillo regular.

Observación 3.18. Observemos que la condición de regularidad relativa corresponde a la lisitud de las fibras de la familia de curvas $X \rightarrow S$, pues para cada $s \in S$ es $X_{s}-x_{s}=$ $\operatorname{Spec} A_{s}$.

Teorema 3.19. Un punto $W \in \operatorname{Gr}(V)(S)$ está en la imagen del morfismo de Krichever para el functor $\mathcal{U}^{\infty}$ si y sólo si $A_{W} \in G r(k((t)))(S), A_{W} \neq \mathcal{O}_{S}$ y es un anillo relativamente regular sobre $S$.

\section{Demostración.}

$\Rightarrow)$ Si $W$ está en la imagen del morfismo de Krichever es

$$
W=\lim _{\text {促 }} E(n D)
$$

donde $\pi: X \rightarrow S$ es una familia propia y plana de curvas íntegras y lisas. Además $A=$ $\lim _{\longrightarrow} \pi_{*} \mathcal{O}_{X}(n D)$ es también un punto de $\operatorname{Gr}(k((z)))(S)$ que es una sub- $\mathcal{O}_{S}$-álgebra de $\overrightarrow{\mathcal{O}_{S}}((z))$ íntegra y relativamente regular, tal que $W$ es $A$-módulo de rango $n$ y que, en virtud del Lema 3.8 verifica automáticamente la condición de platitud de la Definición 3.9 Por maximalidad $A \subseteq A_{W}$. Se concluye entonces, por el Teorema 3.15, que $A=A_{W} \in$ $\operatorname{Gr}(k((z)))(S)$.

$\Leftrightarrow) \mathrm{Si} A_{W} \in \operatorname{Gr}(k((z)))(S)$, como es una sub- $\mathcal{O}_{S}$-álgebra de $\mathcal{O}_{S}((z))$ no trivial, por el Apéndice $\mathrm{B}, A_{W}$ define una familia propia y plana de curvas íntegras $\pi: X \rightarrow S$ y una sección $\sigma$ de $\pi$ de modo que $D=\sigma(S)$ es un divisor de Cartier liso de grado relativo uno. Además $X \rightarrow S$ es una familia de curvas lisas ya que $A_{W}$ es relativamente regular.

Sabemos que $X=\operatorname{Proj} \mathcal{A}$, con $\mathcal{A}:=\oplus_{i \geq 0} x_{1}^{i} A_{W}^{(i)}$ y $A_{W}^{(i)}=A_{W} \cap z^{-i} \mathcal{O}_{S}[[z]]$. Se construye un haz quasi-coherente $E$ sobre $X$ como el haz de localizaciones homogéneas del $\mathcal{A}$-módulo graduado:

$$
\mathcal{W}=\oplus_{i} W^{(i)},
$$

con $W^{(i)}=W \cap z^{-i} \mathcal{O}_{S}[[z]]^{n}$. Además $\mathcal{W}$ es plano sobre $S$ (por ser $W$ un punto de la Grassmanniana y en virtud del Lema 3.8, luego también lo es $E$.

Los teoremas 2.4.3, 2.4.4 y 2.4.5 de [2] demuestran que $E$ es un haz de localmente libre sobre $X$ haciendo una aclaración: 
En [2] se parte de una curva $X$ sobre $k$, luego al cambiar de base al $k$-esquema $S$ se obtiene una familia de curvas $X \times_{k} S$ sobre $S$, de modo que para cada punto cerrado $s \in S$ las fibras $X_{s}$ son la propia curva $X$, luego $s i \cap_{n} \mathfrak{m}_{p}^{n}=0$, al cambiar de base a $S$ esto también ocurre (esto se utiliza en el teorema 2.4.5 de [2]). En nuestro caso tenemos una familia de curvas $X$ sobre $S$ posiblemente distintas, luego aunque en cada fibra sea $\cap_{n} \mathfrak{m}_{p}^{n}=0$, no tendría por que pasar que $\cap_{n} I_{D}^{n}=0$ (donde $I_{D}$ es el ideal del divisor $D$ ). Veamos que sin embargo esto ocurre. En efecto, hemos recuperado el divisor $D$ como el subesquema cerrado de $X$ definido por el ideal $x_{1} \mathcal{A}$, luego:

$$
\cap_{n} I_{D}^{n}=\cap_{n} x_{1}^{n} \mathcal{A}
$$

tenemos que ver que $\cap_{n} x_{1}^{n} \mathcal{A}=0$. Ahora bien, todo elemento $a \in \mathcal{A}=\oplus_{i \geq 0} x_{1}^{i} A_{W}^{(i)}$ puede escribirse:

$$
a=a_{0}+x_{1} a_{1}+x_{1}^{2} a_{2}+\cdots
$$

con $a_{i} \in A_{W}^{(i)}$, y $a \in x_{1}^{m} \mathcal{A}$ si y sólo si $a_{0}=\cdots=a_{m-1}=0$. Sea $a \neq 0$ dado por:

$$
a=x_{1}^{m} a_{m}+x_{1}^{m+1} a_{m+1}+\cdots
$$

con $a_{m} \neq 0$, entonces $a \in x_{1}^{m} \mathcal{A}$ y $a \notin x_{1}^{m+1} \mathcal{A}$, luego:

$$
a \notin \cap_{n} x_{1}^{n} \mathcal{A}
$$

y por tanto $0=\cap_{n} x_{1}^{n} \mathcal{A}$.

Por último, $E$ tiene rango $n$ sobre $X$ en virtud del Corolario 3.12 y para obtener una trivialización formal a lo largo del divisor $D$ no tenemos más que tensorializar por $E$ en la siguiente sucesión exacta:

$$
0 \rightarrow \mathcal{O}_{X}(-n D) \rightarrow \mathcal{O}_{X} \rightarrow \mathcal{O}_{X} / \mathcal{O}_{X}(-n D) \rightarrow 0
$$

Es fácil ahora comprobar que también se tiene el siguiente teorema de caracterización.

Teorema 3.20. Una pareja de puntos $(W, A) \in \operatorname{Gr}(V)(S) \times \operatorname{Gr}(k((z)))(S)$ está en la imagen del morfismo de Krichever para la definición 3.5 si y sólo si $A$ es una sub- $\mathcal{O}_{S^{-}}$ álgebra de $\mathcal{O}_{S}((z))$ relativamente regular y $W$ es A-módulo.

\subsection{Representabilidad.}

En este apartado demostramos que el espacio de moduli $\mathcal{U}^{\infty}$ es representable por un $k$-esquema. 
Lema 3.21. ([2]) Sea $S$ un k-esquema y sea $\mathcal{F}$ un haz de $\mathcal{O}_{S}$-módulos sobre la categoría de $S$-esquemas. Sean $\mathcal{F}_{1}$ y $\mathcal{F}_{2}$ dos subhaces cuasi-coherentes de $\mathcal{F}$ tales que localmente $\mathcal{F} / \mathcal{F}_{2} \simeq \lim _{i} L_{i}$, donde los $L_{i}$ son haces coherentes y libres.

Entonces los puntos $f: S^{\prime} \rightarrow S$ tales que $f^{*}\left(\mathcal{F}_{1}\right) \subseteq f^{*}\left(\mathcal{F}_{2}\right)$, como subhaces de $f^{*}(\mathcal{F})$, son los puntos de un cerrado de $S$.

Demostración. Como la cuestión es local puede suponerse que $S$ es afín, $S=\operatorname{Spec} A$. Entonces $\mathcal{F} / \mathcal{F}_{2} \simeq \lim _{i} L_{i}$ y $\mathcal{F} \simeq \mathcal{F}_{2} \oplus \lim _{i} L_{i}$ donde los $L_{i}$ son $A$-módulos libres. Se deduce además que para un morfismo $f: S^{\prime} \rightarrow S$ es $f^{*}\left(\mathcal{F} / \mathcal{F}_{2}\right)=f^{*}(\mathcal{F}) / f^{*}\left(\mathcal{F}_{2}\right)$.

Dado el morfismo inyectivo de haces:

$$
j:\left(\mathcal{F}_{1}+\mathcal{F}_{2}\right) / \mathcal{F}_{2} \hookrightarrow \mathcal{F} / \mathcal{F}_{2} \simeq{\underset{\lim }{i}}_{i} L_{i}
$$

todo punto $f: S^{\prime} \rightarrow S$ induce un homomorfismo de haces sobre $S^{\prime}$ :

$$
f^{*}(j): f^{*}\left(\left(\mathcal{F}_{1}+\mathcal{F}_{2}\right) / \mathcal{F}_{2}\right) \rightarrow f^{*}\left(\mathcal{F} / \mathcal{F}_{2}\right)=f^{*}(\mathcal{F}) / f^{*}\left(\mathcal{F}_{2}\right),
$$

cuya imagen vale:

$$
\operatorname{Im}\left(f^{*}(j)\right)=\left(f^{*}\left(\mathcal{F}_{1}\right)+f^{*}\left(\mathcal{F}_{2}\right)\right) / f^{*}\left(\mathcal{F}_{2}\right) .
$$

Luego el conjunto de los puntos $f: S^{\prime} \rightarrow \operatorname{Spec} A$ tales que $f^{*}\left(\mathcal{F}_{1}\right) \subseteq f^{*}\left(\mathcal{F}_{2}\right)$ está formado por lo puntos $f$ que verifican:

$$
\operatorname{Im}\left(f^{*}(j)\right)=\left(f^{*}\left(\mathcal{F}_{1}\right)+f^{*}\left(\mathcal{F}_{2}\right)\right) / f^{*}\left(\mathcal{F}_{2}\right)=f^{*}\left(\mathcal{F}_{2}\right) / f^{*}\left(\mathcal{F}_{2}\right)=0
$$

Denotemos $M=\left(\mathcal{F}_{1}+\mathcal{F}_{2}\right) / \mathcal{F}_{2}$, que es un $A$-módulo, y escribamos $j: M \hookrightarrow \varliminf_{i} L_{i}$. Para cada $m \in M$ se tiene que $j(m)=\lim _{i}\left(a_{i}(m)\right)$ con $a_{i}(m) \in L_{i} \simeq A^{i_{r}}$, es decir:

$$
a_{i}(m)=\left(a_{i_{1}}(m), \ldots, a_{i_{r}}(m)\right) \quad \text { donde } a_{i_{k}}(m) \in A \text {. }
$$

Entonces, un punto $f: S^{\prime} \rightarrow \operatorname{Spec} A$ verifica que $\operatorname{Im}\left(f^{*}(j)\right)=0$ si y sólo si, para todo $m \in M$ las funciones $a_{i_{k}}(m)$ son todas nulas (pensadas como funciones sobre $S^{\prime}$ a través de $f$ ). Esto ocurre si y sólo si $f$ factoriza a través de:

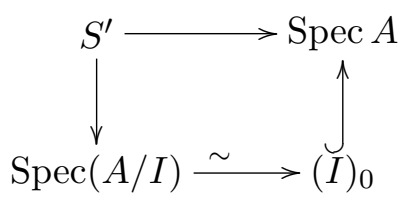

donde $I$ es el ideal de $A$ generado por las $a_{i_{k}}(m)$, con lo que se concluye.

Teorema 3.22. El functor de moduli $\mathcal{U}^{\infty}$ es representable por un subesquema localmente cerrado de $\operatorname{Gr}(V) \times \operatorname{Gr}(k((z)))$. 
Demostración. El Teorema 3.20 demostró la existencia de un morfismo inyectivo de functores:

$$
\mathrm{Kr}_{2}: \mathcal{U}^{\infty} \hookrightarrow \operatorname{Gr}(V) \times \operatorname{Gr}(k((z))) .
$$

Por otro lado, dado que tanto $\operatorname{Gr}(V)$ como $\operatorname{Gr}(k((z)))$ son representables por $k$-esquemas (véase el Teorema A.20, existen subespacios discretos universales:

$$
\begin{gathered}
W_{G} \in \operatorname{Gr}(V)(\operatorname{Gr}(V)) \\
A_{G} \in \operatorname{Gr}(k((z)))(\operatorname{Gr}(k((z))))
\end{gathered}
$$

En estos términos, un punto proviene de $\mathcal{U}^{\infty}$ si y sólo si $A_{G} \cdot A_{G}=A_{G}, A_{G} \cdot W_{G} \subseteq$ $W_{G}$ y $A_{G}$ relativamente regular. Tomando entonces $S=\operatorname{Gr}(V) \times \operatorname{Gr}(k((z))), \mathcal{F}=V_{S}$, $\mathcal{F}_{1}=A_{G} \cdot W_{G}$ y $\mathcal{F}_{2}=W_{G}$ en el lema anterior se sigue que los puntos $f: S^{\prime} \rightarrow S$ tales que $f^{*}\left(\mathcal{F}_{1}\right) \subseteq f^{*}\left(\mathcal{F}_{2}\right)$, como subhaces de $f^{*}(\mathcal{F})$, son los puntos de un cerrado $C$ de $S=\operatorname{Gr}(V) \times \operatorname{Gr}(k((z)))$. Los puntos de $C$ que proviene de familias de curvas lisas forman un abierto dentro de este cerrado.

\subsection{Caso singular.}

Como al principio de este capítulo, podemos definir el functor de moduli que sobre un $k$-esquema $S$ parametriza datos $(X, D, z, E, \phi)$, donde ahora las fibras geométricas de la familia $X \rightarrow S$ pueden ser curvas singulares, $D$ sigue siendo liso y $E$ es un haz libre de torsión de rango $n$ sobre $X$. Del mismo modo podemos definir el morfismo de Krichever en $\operatorname{Gr}\left(k((z))^{n}\right)$ (pues $D$ es liso).

Se plantea entonces el problema de que el morfismo de Krichever pueda dejar de ser inyectivo. Veámoslo con el siguiente ejemplo. Sean $(X, x, z, E, \phi)$ y $\left(X^{\prime}, x^{\prime}, z^{\prime}, E^{\prime}, \phi^{\prime}\right)$ dos puntos racionales y supongamos que existe un único punto singular $p \neq x$ en $X$ de modo que $X^{\prime}$ es la normalización de $X$. Denotemos $f: X^{\prime} \rightarrow X$ al morfismo birracional de normalización y supongamos que $x^{\prime}=f^{-1}(x)$. Supongamos además que existe un isomorfismo $E \simeq f_{*} E^{\prime}$ compatible con las trivializaciones formales $\phi$ y $\phi^{\prime}$. Entonces se tiene que:

$(z \circ \phi) \mathrm{H}^{0}(X-x, E)=\left(z \circ f_{*} \phi^{\prime}\right) \mathrm{H}^{0}\left(X-x, f_{*} E^{\prime}\right)=\left(z^{\prime} \circ \phi^{\prime}\right) \mathrm{H}^{0}\left(X^{\prime}-p^{\prime}, E^{\prime}\right) \in \operatorname{Gr}\left(k((z))^{n}\right)(k)$,

es decir, los puntos de $\operatorname{Gr}\left(k((z))^{n}\right)$ que definen los datos $(X, p, z, E, \phi)$ y $\left(X^{\prime}, p^{\prime}, z^{\prime}, E^{\prime}, \phi^{\prime}\right)$ vía el morfismo de Krichever, son iguales, fallando en consecuencia la inyectividad del morfismo de Krichever. Esto sugiere dar la siguiente definición (veáse [42]):

Definición 3.23. Diremos que un dato $(X, D, z, E, \phi)$ sobre $k$ es maximal cuando cada vez que exista $\left(X^{\prime}, D^{\prime}, z^{\prime}, E^{\prime}, \phi^{\prime}\right)$ sobre $k$ y un morfismo $f: X^{\prime} \rightarrow X$ birracional tal que $\pi^{\prime-1}(D)=D^{\prime}$ y $E \simeq f_{*} E^{\prime}$ de modo compatible con las trivializaciones formales, entonces $X \simeq X^{\prime}$. Diremos que un dato sobre un $k$-esquema $S$ es maximal cuando lo sea para cada punto cerrado $s \in S$. Denotaremos $\widetilde{\mathcal{U}}^{\infty}$ al moduli de datos maximales.

Motivados por esta definición introducimos la siguiente: 
Definición 3.24. Diremos que una pareja:

$$
(W, A) \in \operatorname{Gr}(V)(k) \times \operatorname{Gr}(k((z)))(k)
$$

tal que $A \cdot W \subseteq W$ es maximal cuando, cada vez que exista $A^{\prime} \subset k((z))$ tal que $A^{\prime} \cdot W \subseteq W$, entonces $A^{\prime} \subseteq A$. Diremos que una pareja sobre $S$ es maximal cuando lo sea para cada punto cerrado de $S$.

Observación 3.25. Puede generalizarse la noción de cambio de base del estabilizador para datos maximales y se verifican también en este caso los resultados 3.14 y 3.15 . Es decir, cuando la pareja $(A, W)$ es maximal, entonces $A_{W}$ sigue siendo el estabilizador después de cualquier cambio de base.

Teorema 3.26. Sea $S$ un k-esquema. Los puntos $\widetilde{\mathcal{U}}^{\infty}(S)$ se corresponden, vía el morfismo de Krichever:

$$
\tilde{\mathrm{K}} \mathrm{:}: \tilde{\mathcal{U}}^{\infty} \rightarrow \operatorname{Gr}(V)(S),
$$

con los $W \in \operatorname{Gr}(V)(S)$ tales que $A_{W} \in \operatorname{Gr}(k((z)))(S)$ y la pareja $\left(W, A_{W}\right)$ es maximal.

Teorema 3.27. Sea $S$ un k-esquema. Los puntos $\widetilde{\mathcal{U}}^{\infty}(S)$ se corresponden, vía el morfismo de Krichever:

$$
\tilde{\mathrm{Kr}}_{2}: \tilde{\mathcal{U}}^{\infty} \rightarrow \operatorname{Gr}(V)(S) \times \operatorname{Gr}(k((z)))(S),
$$

con las parejas maximales $(W, A) \in \operatorname{Gr}(V)(S) \times \operatorname{Gr}(k((z)))(S)$ tales que $A \cdot W \subseteq W$.

Teorema 3.28. El functor de moduli $\tilde{\mathcal{U}}^{\infty}$ es representable por un subesquema cerrado de $\operatorname{Gr}(V) \times \operatorname{Gr}(k((z)))$.

Demostración. Razonando como en el Teorema 3.22 obtenemos un cerrado $C$ en $\operatorname{Gr}(V) \times$ $\operatorname{Gr}(k((z)))$ formado por las parejas $(W, A)$ tales que $A \cdot A=A$ y $A \cdot W \subseteq W$. Dentro de este cerrado, la condición de maximalidad se traduce en $A=A_{W}$. Tenemos así dos puntos de la Grassmanniana de $k((z)), A$ y $A_{W}$ tales que $A \subseteq A_{W}$, luego serán iguales si tienen el mismo índice (veáse la Observación 3.7) y por lo tanto $\widetilde{\mathcal{U}}^{\infty}$ es representable por el corte de $C$ con unas cuantas componentes conexas de $\operatorname{Gr}(V) \times \operatorname{Gr}(k((z)))$, que es cerrado. 


\section{Capítulo 4}

\section{Pares de Higgs y Grassmanniana Infinita.}

Este capítulo pretende caracterizar la imagen del morfismo de Krichever para el moduli de pares de Higgs sobre una curva lisa con trivializaciones formales, y representar dicho moduli como un subesquema dentro de una Grassmanniana infinita. Dado un punto de este espacio de moduli, resulta claro que la Grassmanniana a la que debemos enviar el fibrado es $\operatorname{Gr}\left(k((z))^{n}\right.$. No obstante, no es tan trivial imaginarse en qué espacio debemos codificar el campo de Higgs, y por tanto, deducir en qué espacio debe valorar el morfismo de Krichever. El ingrediente clave para definirlo consiste en introducir una fibración de Grassmannianas infinitas sobre un espacio afín, que esencialmente mide la variación del polinomio característico del campo de Higgs, y que no es sino un análogo formal a la llamada base de Hitchin.

\subsection{Preliminares.}

Comenzamos este capítulo hablando brevemente de algunos de los ingredientes que vamos a necesitar de la teoría de pares de Higgs. Trabajaremos sobre un cuerpo $k$ algebraicamente cerrado de característica nula.

Denotemos por $\mathcal{U}_{X}$ el moduli de fibrados vectoriales de rango $n$ sobre una curva propia, lisa e íntegra $X$. Dar un par de Higgs en $X$ consiste en dar un punto $E \in \mathcal{U}_{X}$ junto con un morfismo de haces de $\mathcal{O}_{X}$-módulos:

$$
\varphi: E \rightarrow E \otimes \omega_{X} .
$$

Es bien sabido que el espacio tangente al moduli de fibrados vale:

$$
\mathrm{T}_{E} \mathcal{U}_{X} \stackrel{\sim}{\longrightarrow} \mathrm{H}^{1}\left(X, \mathcal{E} \operatorname{nd}_{X} E\right)
$$

y por dualidad de Serre:

$$
\mathrm{T}_{E}^{*} \mathcal{U}_{X} \simeq \mathrm{H}^{0}\left(X, \mathcal{E} \operatorname{nd}_{X} E \otimes \omega_{X}\right)
$$


Es decir, el espacio cotangente al moduli de fibrados parametriza pares de Higgs en $X$. Un hecho importante es la existencia del llamado morfismo de Hitchin:

$$
\begin{aligned}
H: \mathrm{T}^{*} \mathcal{U} & \rightarrow \oplus_{i=1}^{n} \mathrm{H}^{0}\left(X, \omega_{X}^{\otimes i}\right) \\
(E, \varphi) & \mapsto \operatorname{ch}(\varphi)=\left(\operatorname{Tr}(\varphi), \operatorname{Tr}\left(\Lambda^{2} \varphi\right), \ldots, \operatorname{Tr}\left(\Lambda^{n} \varphi\right)\right)
\end{aligned}
$$

donde $\operatorname{ch}(\varphi)$ son los coeficientes característicos de $\varphi$. Su importancia radica en el hecho de que, para un punto genérico $a=\left(a_{1}, \ldots, a_{n}\right) \in \oplus_{i=1}^{n} \mathrm{H}^{0}\left(X, \omega_{X}^{\otimes i}\right)$, la fibra del morfismo de Hitchin corresponde (uno a uno) a haces de línea en otra curva $X_{a}$, llamada curva espectral. Describimos brevemente la construcción de $X_{a}$ (véase [21],[7],[45]). Consideremos el fibrado cotangente:

$$
\mathrm{T}^{*} X:=\operatorname{Spec} \mathcal{S}^{\bullet} \omega_{X}^{-1}
$$

donde $\mathcal{S}^{\bullet} \omega_{X}^{-1}$ es el álgebra simétrica de $\omega_{X}^{-1}$. Sea $\mathcal{I}_{a}$ el haz de ideales de $\mathcal{S}^{\bullet} \omega_{X}^{-1}$ generado por la imagen del morfismo de haces de $\mathcal{O}_{X}$-módulos:

$$
\begin{aligned}
\omega_{X}^{-n} & \rightarrow \mathcal{S}^{\bullet} \omega_{X}^{-1}=\oplus_{i \geq 0} \omega_{X}^{\otimes-i} \\
\xi & \mapsto\left(\xi \otimes a_{n}, \xi \otimes a_{n-1}, \ldots, \xi \otimes a_{1}, \xi, 0,0, \ldots\right)
\end{aligned}
$$

El cociente $\mathcal{S}^{\bullet} \omega_{X}^{-1} / \mathcal{I}_{a}$ es un haz de $\mathcal{O}_{X}$-álgebras y definimos la curva espectral como los ceros de $\mathcal{I}_{a}$ en $\mathrm{T}^{*} X$, es decir:

$$
X_{a}=\operatorname{Spec} \mathcal{S}^{\bullet} \omega_{X}^{-1} / \mathcal{I}_{a}
$$

El morfismo:

$$
\pi: X_{a} \rightarrow X
$$

es un revestimiento (posiblemente ramificado) de grado $n$, donde $n$ es el rango de $E$, y para un punto genérico $a, X_{a}$ es una curva no singular. Para $a$ genérico, la biyección entre $\operatorname{Jac}\left(X_{a}\right)$ y $H^{-1}(a)$ es la siguiente. El campo de Higgs $\varphi$ puede interpretarse como un morfismo de haces de $\mathcal{O}_{X}$-módulos:

$$
\omega_{X}^{-1} \rightarrow E \otimes E^{*}
$$

o equivalentemente, como un morfismo de $\mathcal{O}_{X}$-álgebras:

$$
\mathcal{S}^{\bullet} \omega_{X}^{-1} \rightarrow E \otimes E^{*} .
$$

Por el teorema de Hamilton-Cayley, el morfismo anterior factoriza por $\mathcal{O}_{X_{a}}=\mathcal{S}^{\bullet} \omega_{X}^{-1} / \mathcal{I}_{a}$ si y sólo si $\operatorname{ch}(\varphi)=a$. Luego dado un punto en $H^{-1}(a)$, esto es, dado un par de Higgs $(E, \varphi)$ con $\operatorname{ch}(\varphi)=a$, podemos pensar $E$ como un haz de línea $L$ en $X_{a}$. Claramente $\pi_{*} L=E$.

Recíprocamente, dado un haz de línea $L$ en $X_{a}$, sea $E=\pi_{*} L . E$ es un fibrado de rango $n$ en $X$ y admite una estructura de $\pi_{*} \mathcal{O}_{X_{a}}$-módulo que induce:

$$
\mathcal{S}^{\bullet} \omega_{X}^{-1} \rightarrow \mathcal{S}^{\bullet} \omega_{X}^{-1} / \mathcal{I}_{a} \rightarrow E \otimes E^{*}
$$

Pero esto es equivalente a dar un morfismo de $\mathcal{O}_{X}$-módulos $\omega_{X}^{-1} \rightarrow E \otimes E^{*}$ tal que $\operatorname{ch}(\varphi)=$ $a$. 


\subsection{Moduli de pares de Higgs con trivializaciones formales.}

En lo que sigue, supondremos fijos los siguientes datos:

- $X$ curva proyectiva, lisa e íntegra sobre $k$.

- $x \in X$.

- $t: \widehat{\mathcal{O}}_{X, x} \simeq k[[z]]$ una trivialización formal de $X$ en $x$.

Por ser $X$ una curva lisa, el haz dualizante $\omega_{X}$ es de línea y la trivialización formal $t$ induce de modo natural una trivialización formal de $\omega_{X}$ en $x$ ([34]):

$$
d t: \widehat{\omega}_{X, x} \simeq k[[z]] d z
$$

(obviaremos, cuando no sea necesario, el generador $d z$ ). Nuestro objetivo es definir un espacio de moduli que parametrice ternas $(E, \varphi, \phi)$ formadas por:

- Un fibrado $E$ de rango $n$ sobre $X$.

- Un campo de Higgs $\varphi: E \rightarrow E \otimes \omega_{X}$.

- Una trivialización formal $\phi$ de $E$ en $x, \phi: \widehat{E}_{x} \simeq \widehat{\mathcal{O}}_{X, x}^{n}$.

Veremos que hemos de imponer una cierta compatibilidad entre la trivialización formal y el campo de Higgs.

\subsubsection{Definición y representabilidad.}

Denotemos $\widehat{\varphi}$ a la completación en $x$ del campo de Higgs $\varphi$. Dada la terna $(X, x, z)$ y una trivialización formal $\phi$ de $E$ en $x$, los coeficientes $a_{i}$ del polinomio característico $p_{\widehat{\varphi}}(T)$ de $\widehat{\varphi}$ yacen en $k[[z]]$.

Definición 4.1. Se define el functor $\mathcal{H}_{\text {iggs }}^{\infty}{ }_{X}^{\infty}$ como el hacificado de:

$$
\begin{aligned}
& \mathcal{C}_{k-e s q} \rightsquigarrow \mathcal{C}_{\text {sets }} \\
& S \mapsto\{(E, \phi, \varphi)\} / \sim
\end{aligned}
$$

donde:

1. $E$ es un fibrado de rango $n$ sobre $X \times S$.

2. $\varphi: E \rightarrow E \otimes \omega_{X \times S / S}$ es un campo de Higgs relativo, es decir, un morfismo $\mathcal{O}_{X \times S^{-}}$ módulos. 
3. $\phi$ es una trivialización formal de $E$ a lo largo de $x \times S$ :

$$
\phi: \widehat{E}_{x \times S} \simeq \widehat{\mathcal{O}}_{x \times S}^{n}
$$

para la que existe una matriz:

$$
M=\left(\begin{array}{cccccc}
0 & & & & 0 & (-1)^{n+1} a_{n} \\
1 & \cdot & & & \cdot & (-1)^{n} a_{n-1} \\
\cdot & 1 & \cdot & & \cdot & \cdot \\
\cdot & & \cdot & \cdot & \cdot & \cdot \\
\cdot & & & \cdot & 0 & -a_{2} \\
0 & \cdot & & 0 & 1 & a_{1}
\end{array}\right)
$$

(donde $\left(a_{1}, \ldots, a_{n}\right) \in \mathcal{O}_{S}[[z]]^{n}$ ) haciendo compatible el siguiente diagrama:

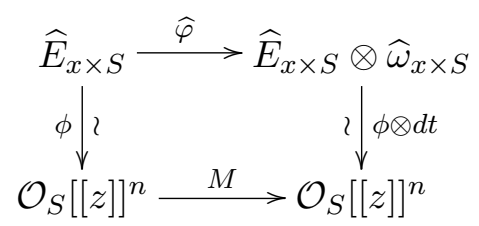

4. Diremos que $(E, \varphi, \phi) \sim\left(E^{\prime}, \varphi^{\prime}, \phi^{\prime}\right)$ cuando exista un isomorfismo de fibrados $f: E \simeq E^{\prime}$ compatible con todos los datos, es decir:

- $f$ es compatible con $\phi \mathrm{y} \phi^{\prime} \mathrm{y}$, por lo tanto, tenemos un diagrama conmutativo:

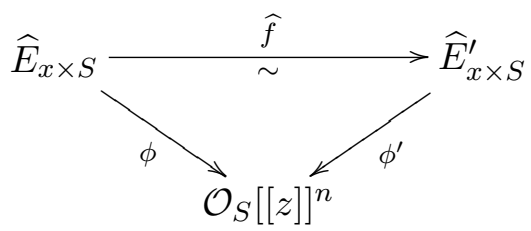

- $f$ es compatible con $\varphi$ y $\varphi^{\prime}$, o sea, el siguiente diagrama es conmutativo:

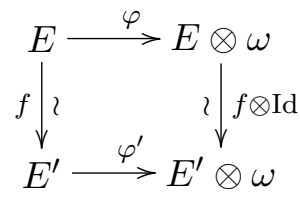

Observación 4.2. Dado $(E, \phi, \varphi) \in \mathcal{H}_{\text {iggs }}^{\infty}(S)$, los elementos $\left(a_{1}, \ldots, a_{n}\right)$ de la tercera condición son, forzosamente, los coeficientes del polinomio característico formal definido por $\widehat{\varphi}$. 
Observación 4.3. Cuando dos ternas $(E, \varphi, \phi),\left(E^{\prime}, \varphi^{\prime}, \phi^{\prime}\right)$ son equivalentes, los coeficientes de los polinomios característicos de los campos de Higgs formales son los mismos y se tiene un diagrama conmutativo:

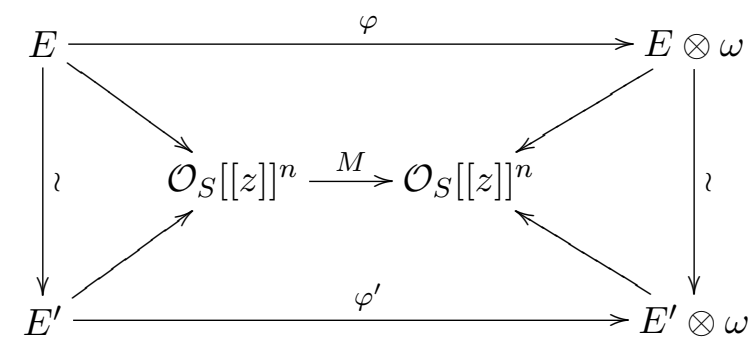

Escribiremos $\mathcal{U}_{X}$ para el stack de fibrados vectoriales sobre $X$ y $\mathcal{U}_{X}^{\infty}$ al espacio de moduli de fibrados vectoriales sobre $X$ con trivialización formal (recordemos que es último espacio de moduli puede obtenerse como límite del espacio de moduli de fibrados con estructura de nivel,[43, 4eme partie], y el Teorema 3.22 demuestra en particular que es un moduli fino). Es fácil ver que para todo fibrado vectorial $E$ sobre $X$, podemos escoger una trivialización formal en un punto marcado $x \in X, \phi: \widehat{E}_{x} \simeq \widehat{\mathcal{O}}_{X, x}^{n}$. Es decir, podemos elegir un punto en $\mathcal{U}_{X}^{\infty}$.

Denotemos $\mathcal{H i g g s}_{X}$ al stack de pares de Higgs sobre $X$.

Proposición 4.4. Sea $S$ un k-esquema. Dado $(E, \varphi) \in \mathcal{H} \operatorname{iggs}_{X}(S)$, existe una trivialización formal de $E$ a lo largo de $x \times S$ que satisface la condición de compatibilidad de la Definición 4.1

Demostración. Sea $(E, \varphi)$ un punto en $\mathcal{H}_{\text {iggs }}(S)$ y fijemos una trivialización formal $\phi$ de $E$ en $x \times S$. Tomando $\left(a_{1}, \ldots, a_{n}\right) \in \mathcal{O}_{S}[[z]]^{n}$ como los coeficientes característicos de la completación $\widehat{\varphi}$ del campo de Higgs en $x \times S$, puede verse fácilmente que $\phi$ es compatible con $\widehat{\varphi}$ en el sentido de la Definición 4.1 .

Teorema 4.5. El functor Higgs $_{X}^{\infty}$ es representable por un k-esquema.

Demostración. Dado que $\mathcal{U}_{X}^{\infty}$ es un moduli fino, existe el objeto universal $(\mathcal{E}, \Phi)$, donde $\mathcal{E}$ es el fibrado universal de rango $n$ sobre $X \times \mathcal{U}_{X}^{\infty}$ y $\Phi$ una trivialización formal del mismo a lo largo de $\{x\} \times \mathcal{U}_{X}^{\infty}$.

Consideremos el fibrado vectorial $\mathbb{V}$ asociado al siguiente haz localmente libre sobre $X \times \mathcal{U}_{X}^{\infty}$ :

$$
\mathcal{H o m}_{X \times \mathcal{U}_{X}^{\infty}}(\mathcal{E}, \mathcal{E} \otimes \omega)
$$

siendo $\omega$ el pullback de $\omega_{X}$ a $X \times \mathcal{U}_{X}^{\infty}$.

Se tiene que dar un punto de $\mathbb{V}$ con valores en un $k$-esquema $S, g: S \rightarrow \mathbb{V}$, equivale a dar una terna $\left(E_{S}, \varphi_{S}, \phi_{S}\right)$, donde $\left(E_{S}, \phi_{S}\right)$ es el punto de $\mathcal{U}_{X}^{\infty}$ con valores en $S$ dado por la composición:

$$
S \rightarrow \mathbb{V} \rightarrow X \times \mathcal{U}_{X}^{\infty} \stackrel{p_{2}}{\longrightarrow} \mathcal{U}_{X}^{\infty}
$$


(siendo $p_{2}$ la proyección en el segundo factor) y $\varphi_{S}$ es el punto con valores en $S$ definido por $g$.

De la Definición 4.1 se sigue que los puntos de $\mathcal{H}$ iggs ${ }_{X}^{\infty}$ con valores en $S$, son los puntos de $\mathbb{V}$ con valores en $S,\left(E_{S}, \varphi_{S}, \phi_{S}\right)$, tales que la composición:

$$
\mathcal{O}_{S}[[z]]^{n} \stackrel{\phi_{S}^{-1}}{\longrightarrow} \widehat{E}_{S, x \times S} \stackrel{\widehat{\varphi}_{S}}{\longrightarrow} \widehat{E}_{S, x \times S} \otimes \widehat{\omega}_{x \times S} \stackrel{\phi_{S} \times d t_{S}}{\longrightarrow} \mathcal{O}_{S}[[z]]^{n}
$$

viene dada por una matriz del tipo:

$$
\left(\begin{array}{cccccc}
0 & \cdot & & \cdot & 0 & (-1)^{n+1} a_{n} \\
1 & 0 & \cdot & & \cdot & (-1)^{n} a_{n-1} \\
0 & 1 & 0 & \cdot & \cdot & \cdot \\
\cdot & \cdot & \cdot & \cdot & \cdot & \cdot \\
\cdot & \cdot & 0 & 1 & 0 & -a_{2} \\
0 & \cdot & \cdot & 0 & 1 & a_{1}
\end{array}\right)
$$

para cualesquiera $\left(a_{1}, \ldots, a_{n}\right) \in \mathcal{O}_{S}[[z]]^{n}$. Puede comprobarse que esta condición define un subesquema cerrado de $\mathbb{V}$.

\subsubsection{Morfismo de Hitchin formal.}

Como ya dijimos, los coeficientes $a_{i}$ del polinomio característico $p_{\widehat{\varphi}}(T)$ de $\widehat{\varphi}$ viven en $k[[z]]$. Introduzcamos entonces un $k$-esquema que parametrice dichos coeficientes.

Sea $\mathbb{A}^{\infty}$ el $k$-esquema definido en [3, Def. 4.13]:

$$
\lim _{l} \mathbb{A}^{l} \simeq \operatorname{Spec} \underset{l}{\lim } k\left[y_{0}, \ldots, y_{l}\right]=\operatorname{Spec} k\left[y_{0}, y_{1}, \ldots\right]
$$

Sea $R$ una $k$-álgebra. La correspondencia:

$$
s_{0}+s_{1} z+\cdots \in R[[z]] \leftrightarrow\left(s_{0}, s_{1}, \ldots\right) \in \mathbb{A}^{\infty}(R)
$$

implica que el functor $\widetilde{k[[z]]}$, que asocia a cada $k$-álgebra $R$ el anillo de series formales $R[[z]]$, es representable por $\mathbb{A}^{\infty}$. De este hecho se deduce la siguiente proposición.

Proposición 4.6. El functor sobre la categoría de k-esquemas:

$$
S \rightsquigarrow \oplus_{i=1}^{n} \mathrm{H}^{0}\left(\widehat{X}_{x} \times S, \widehat{\omega}_{x \times S}^{\otimes i}\right)
$$

es representable por el k-esquema:

$$
\mathbb{A}=\left(\mathbb{A}^{\infty}\right)^{n}=\operatorname{Spec} k\left[y_{0}^{(1)}, \ldots, y_{0}^{(2)}, \ldots, y_{0}^{(n)}, \ldots\right] .
$$

Observación 4.7. Pensaremos los puntos de $\mathbb{A}$ con valores en $S$ como polinomios:

$$
T^{n}-a_{1} T^{n-1}+\cdots+(-1)^{n} a_{n}
$$

con coeficientes en $\mathcal{O}_{S}[[z]]$. 
Definido el functor de pares de Higgs con trivialización formal, nos proponemos dar una versión formal del morfismo de Hitchin:

$$
H: \mathrm{T}^{*} \mathcal{U} \rightarrow \oplus_{i=1}^{n} \mathrm{H}^{0}\left(X, \omega_{X}^{\otimes i}\right)
$$

Definición 4.8. Se define el morfismo de Hitchin formal:

$$
\mathcal{H}_{\infty}: \mathcal{H i g g s}_{X}^{\infty} \rightarrow \mathbb{A}
$$

como el morfismo de esquemas que asocia a cada triple $(E, \phi, \varphi) \in \mathcal{H}_{\text {iggs }}^{\infty}(S)$ los coeficientes característicos:

$$
\left(a_{1}, \ldots, a_{n}\right) \in \mathbb{A}(S), \quad a_{i}=\operatorname{Tr}\left(\Lambda^{i} \widehat{\varphi}\right)
$$

del campo de Higgs formal $\widehat{\varphi}$.

\subsubsection{Morfismo de Krichever.}

Denotemos $V=k((z))^{n}$ y $V^{+}=k[[z]]^{n}$ y recordemos que la Grassmanniana infinita $\operatorname{Gr}(V)$ asociada a la pareja $\left(V, V^{+}\right)$es el $k$-esquema infinito cuyos puntos racionales son subespacios vectoriales $W$ de $V$ tales que $W \cap V^{+}$y $V / W+V^{+}$son espacios vectoriales de dimensión finita (véase el apéndice A).

Recordemos también (véase [2], [28], o el capítulo 3) que el morfismo de Krichever:

$$
\mathcal{U}_{X}^{\infty} \rightarrow \operatorname{Gr}(V)
$$

se define mandando $(E, \phi)$ a $(t \circ \phi) \varliminf_{\longrightarrow} \pi_{*} E(m)$ y para puntos racionales es:

$$
(E, \phi) \mapsto(t \circ \phi) \mathrm{H}^{0}(X-x, E) .
$$

Definición 4.9. Sea $(E, \phi, \varphi) \in \mathcal{H}_{\text {iggs }}{ }^{\infty}(S)$. Definimos el morfismo de Krichever por:

$$
\begin{aligned}
\mathcal{H i g g s}_{X}^{\infty}(S) & \rightarrow \operatorname{Gr}\left(k((z))^{n}\right)(S) \times \mathbb{A}(S) \\
(E, \phi, \varphi) & \mapsto\left((t \circ \phi){\underset{m}{\lim }}_{*} E(m), p_{\widehat{\varphi}}(T)\right)
\end{aligned}
$$

donde $\pi: X \times S \rightarrow S$ y $p_{\widehat{\varphi}}(T)$ es el polinomio característico del campo de Higgs formal $\widehat{\varphi}$. Para puntos racionales manda $(E, \phi, \varphi)$ a $\left((t \circ \phi) \mathrm{H}^{0}(X-x, E), p_{\widehat{\varphi}}(T)\right)$.

Teorema 4.10. El morfismo de Krichever es inyectivo.

Demostración. Sean $(E, \phi, \varphi),\left(E^{\prime}, \phi^{\prime}, \varphi^{\prime}\right)$ dos puntos de $\mathcal{H} i g g s_{X}^{\infty}$ con valores en el $k$ esquema $S$ y supongamos que:

$$
(t \circ \phi) \underset{m}{\lim _{m}} \pi_{*} E(m)=\left(t \circ \phi^{\prime}\right) \underset{m}{\lim _{m}} \pi_{*} E^{\prime}(m)
$$




$$
p_{\widehat{\varphi}}(T)=p_{\widehat{\varphi}^{\prime}}(T) .
$$

Como el morfismo de Krichever es inyectivo para el moduli $\mathcal{U}_{X}^{\infty}$ de fibrados con trivialización formal (véase [2]), existe un isomorfismo $E \simeq E^{\prime}$ compatible las trivialización formales $\phi$ y $\phi^{\prime}$. Dado que las ambas familias verifican la tercera condición de la Definición 4.1 para la misma matriz $M$ (pues $p_{\widehat{\varphi}}(T)=p_{\widehat{\varphi}^{\prime}}(T)$ ), se deduce que el diagrama:

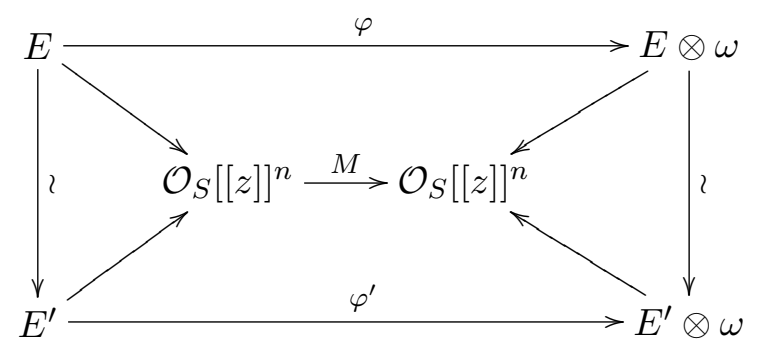

es conmutativo, y por lo tanto $(E, \phi, \varphi) \sim\left(E^{\prime}, \phi^{\prime}, \varphi^{\prime}\right)$ (veáse la Observación 4.3).

Nos disponemos ahora a caracterizar la imagen del morfismo de Krichever. Consideremos la aplicación natural:

$$
V \otimes_{k} k((z)) \rightarrow V=V \otimes_{k((z))} k((z)) .
$$

Si $W$ y $U$ son subespacios vectoriales de $V$ y $k((z))$ respectivamente, denotaremos $W \cdot U$ a la imagen de $W \otimes_{k} U \rightarrow V$.

Proposición 4.11. Sea $L$ un haz de línea en $X$ y $\phi_{L}$ una trivialización formal de $L$ en $x$. Dado un punto racional $\left(E, \phi_{E}\right)$ de $\mathcal{U}_{X}^{\infty}$, sean:

$$
W=\mathrm{H}^{0}(X-x, E) \in \operatorname{Gr}(V), \quad U=\mathrm{H}^{0}(X-x, L) \in \operatorname{Gr}(k((z)))
$$

los puntos definidos por el morfismo de Krichever. Entonces:

$$
W \cdot U=\mathrm{H}^{0}\left(X-x, E \otimes_{\mathcal{O}_{X}} L\right) \in \operatorname{Gr}(V) .
$$

Demostración. Basta demostrar que:

$$
\mathrm{H}^{0}(X-x, E) \otimes_{k} \mathrm{H}^{0}(X-x, L) \rightarrow \mathrm{H}^{0}\left(X-x, E \otimes_{\mathcal{O}_{X}} L\right)
$$

es epiyectiva. En efecto, como $X-x=\operatorname{Spec} A$ es un abierto afín de $X$ y el haz producto tensorial coincide con su prehaz sobre los abiertos afines, se tiene que:

$$
\mathrm{H}^{0}(X-x, E) \otimes_{\mathrm{H}^{0}\left(X-x, \mathcal{O}_{X}\right)} \mathrm{H}^{0}(X-x, L) \stackrel{\sim}{\rightarrow} \mathrm{H}^{0}\left(X-x, E \otimes_{\mathcal{O}_{X}} L\right),
$$

de donde se sigue el enunciado. 
Proposición 4.12. Sea $S$ un k-esquema, $L$ un haz de línea en $X \times S$ y $\phi_{L}$ una trivialización formal del mismo a lo largo de $x \times S$. Dado un punto $\left(E, \phi_{E}\right)$ de $\mathcal{U}_{X}^{\infty}$ con valores en $S$, sean:

$$
U=\underset{m}{\lim } \pi_{*} L(m) \in \operatorname{Gr}(k((z)))(S), \quad W=\underset{m}{\lim _{m}} \pi_{*} E(m) \in \operatorname{Gr}(V)(S)
$$

los puntos que definen por el morfismo de Krichever (donde $\pi: X \times S \rightarrow S$ ). Entonces:

$$
W \cdot U=\underset{m}{\underset{m}{\lim } \pi_{*}}\left(E \otimes_{\mathcal{O}_{X}} L(m)\right) \in \operatorname{Gr}(V)(S) .
$$

En particular se tiene un morfismo:

$$
\begin{aligned}
h_{U}: \mathcal{U}_{X}^{\infty} & \rightarrow \mathcal{U}_{X}^{\infty} \\
W & \mapsto W \cdot U
\end{aligned}
$$

Demostración. Como en la proposición anterior, basta demostrar que el morfismo

$$
\underset{m}{\lim } \pi_{*} E(m) \otimes_{\lim _{m}} \pi_{*} \mathcal{O}_{X}(m) \underset{m}{\lim _{*}} \pi_{*} L(m) \rightarrow \underset{m}{\lim _{m}} \pi_{*}\left(E \otimes \mathcal{O}_{X} L(m)\right)
$$

es isomorfismo. Ahora bien, como es un morfismo de haces, basta que lo sea en sobre los puntos cerrados y esto se sigue de la proposición anterior.

Observación 4.13. Nótese que no tenemos sin embargo, un morfismo de esquemas bien definido:

$$
\begin{aligned}
\operatorname{Gr}(k((z))) \times \operatorname{Gr}(V) & \rightarrow \operatorname{Gr}(V) \\
A \otimes_{k} W & \mapsto A \cdot W,
\end{aligned}
$$

como ilustra el siguiente ejemplo. Sea $A$ el subespacio vectorial de $k((z))$ generado por

$$
1, z^{-1}, z^{-2}, \ldots
$$

$A=k\left[z^{-1}\right]$ es precisamente el anillo del abierto afín $\mathbb{P}^{1}-\{p\}$ de $\mathbb{P}^{1}$, siendo $p$ el punto del infinito. Es decir, $A=\mathrm{H}^{0}\left(\mathbb{P}^{1}-p, \mathcal{O}_{\mathbb{P}^{1}}\right) \in \operatorname{Gr}(k((z)))$ es un punto geométrico.

Sea $W$ el $k$-espacio vectorial generado por $z^{i}+z^{-i}$, que es un punto de $\operatorname{Gr}(k((z)))$ que no viene de datos geométricos. Entonces $z^{-1} W$ está generado por:

$$
1+z^{-2}, z+z^{-3}, z^{2}+z^{-4}, z^{3}+z^{-5}, \ldots
$$

y como $1 \cdot W \subset A \cdot W$ y $z^{-1} W \subset A \cdot W$, podemos obtener cualquier potencia de $z$ en $A \cdot W$, es decir, el $k$-espacio vectorial de dimensión infinita $k[z]$ está contenido en $(A \cdot W) \cap k[[z]]$, de lo que se deduce que $A \cdot W$ no es un punto de $\operatorname{Gr}(k((z)))$. 
Nos proponemos ahora caracterizar la imagen del morfismo de Krichever. Sabemos (véase [28] o el Teorema 3.19] que la imagen del morfismo de Krichever:

$$
\mathcal{U}_{X}^{\infty} \hookrightarrow \operatorname{Gr}(V)
$$

está formada por aquellos puntos $W \in \operatorname{Gr}(V)$ tales que $A \cdot W \subseteq W$ (donde $A=\mathrm{H}^{0}(X-$ $\left.x, \mathcal{O}_{X}\right)$ ). Como el morfismo:

$$
\mathcal{H i g g s}_{X}^{\infty} \hookrightarrow \operatorname{Gr}(V) \times \mathbb{A}
$$

valora en $\mathcal{U}_{X}^{\infty} \times \mathbb{A}$, basta con estudiar la imagen de:

$$
\operatorname{Higgs}_{X}^{\infty} \hookrightarrow \mathcal{U}_{X}^{\infty} \times \mathbb{A}
$$

Proposición 4.14. Sea $S$ un k-esquema, $A_{S}$ el punto de $\operatorname{Gr}(k((z))(S)$ que define la familia de curvas $X \times S \rightarrow S$ por el morfismo de Krichever, y sea $T$ un automorfismo $\mathcal{O}_{S}((z))$-lineal de $\mathcal{O}_{S}((z))^{n}$. Dado $W \in \mathcal{U}_{X}^{\infty}(S)$, se verifican las siguientes condiciones:

1. $T(W) \in \operatorname{Gr}(V)(S)$.

2. $T(W)$ es un $A_{S}$-módulo.

3. Se tiene una igualdad de estabilizadores $A_{T(W)}=A_{W}=A_{S} \in \operatorname{Gr}(k((z)))(S)$.

En consecuencia, $T(W) \in \mathcal{U}_{X}^{\infty}(S)$.

Demostración. La primera condición se sigue por ser $T \mathcal{O}_{S}((z))$-lineal; para la segunda hay que tener en cuenta que $T$ es $\mathcal{O}_{S}((z))$-lineal y que $A_{S} \subset \mathcal{O}_{S}((z))$; para la tercera basta tener en cuenta la cadena de inclusiones:

$$
A_{S} \subseteq A_{T(W)} \subseteq A_{W}
$$

y las propiedades de los estabilizadores (véase el Lema 3.14 y el Teorema 3.15).

Podemos definir ahora el siguiente endomorfismo de $\mathcal{U}_{X}^{\infty}$ :

$$
\begin{aligned}
T: \mathcal{U}_{X}^{\infty} & \rightarrow \mathcal{U}_{X}^{\infty} \\
W & \mapsto T(W) .
\end{aligned}
$$

Teorema 4.15. Sea $\Omega$ el punto de $\operatorname{Gr}(k((z)))(S)$ que define el haz dualizante $\omega_{X \times S / S}$, $(W, p(T)) \in\left(\mathcal{U}_{X}^{\infty} \times \mathbb{A}\right)(S)$ y $T$ el automorfismo $\mathcal{O}_{S}((z))$-lineal de $\mathcal{O}_{S}((z))^{n}$ dado por la matriz:

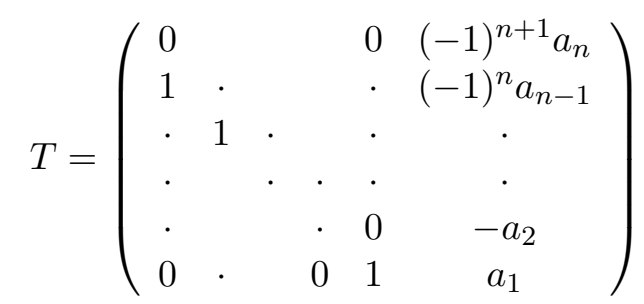


donde $\left(a_{1}, \ldots, a_{n}\right) \in \mathbb{A}(S)$ son los coeficientes de $p(T)$. Entonces, $(W, p(T))$ está imagen del morfismo:

$$
\mathcal{H i g g s}_{X}^{\infty} \hookrightarrow \mathcal{U}_{X}^{\infty} \times \mathbb{A}
$$

si y sólo si la restricción de $T$ a $W$ valora en $W \cdot \Omega$ :

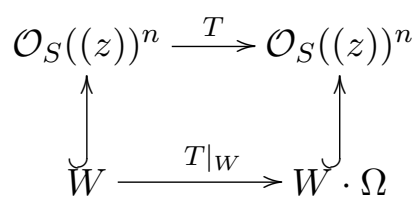

Para puntos cerrados la condición anterior se traduce en $T(W) \subseteq W \cdot \Omega$.

Demostración. El directo se sigue de la definición del morfismo de Krichever. Veamos el recíproco. Sea $(W, p(T)) \in \mathcal{U}_{X}^{\infty}(S) \times \mathbb{A}(S)$ tal que $\left.T\right|_{W}$ valora en $W \cdot \Omega$. Por hipótesis $W$ es geométrico, es decir, $W$ viene por el morfismo de Krichever de un punto en $\mathcal{U}_{X}^{\infty}(S)$. En consecuencia, tenemos un fibrado vectorial $E$ de rango $n$ sobre $X \times S$ y una trivialización formal $\phi$ del mismo a lo largo de $x \times S$. Por la Proposición 4.12 se tiene que $W \cdot \Omega$ es el punto de $\operatorname{Gr}\left(k((z))^{n}\right)(S)$ definido por el haz $E \otimes_{\mathcal{O}_{X}} \omega_{X \times S / S}$, es decir:

$$
W \cdot \Omega=\underset{m}{\lim } \pi_{S, *}\left(E \otimes_{\mathcal{O}_{X}} \omega_{X \times S / S}\right)(m(x \times S)),
$$

luego el morfismo $\left.T\right|_{W}$ se traduce en :

$$
\left.T\right|_{W}: \underset{m}{\lim } \pi_{S, *} E(m(x \times S)) \rightarrow \underset{m}{\lim _{S}} \pi_{S, *}\left(E \otimes_{\mathcal{O}_{X}} \omega_{X \times S / S}\right)(m(x \times S))
$$

donde $\pi_{S}: X \times S \rightarrow S$. Teniendo en cuenta que $T$ es $\mathcal{O}_{S}((z))$-lineal y la correspondencia inversa del morfismo de Krichever, podemos recuperar el campo de Higgs:

$$
E \rightarrow E \otimes \omega_{X \times S / S}
$$

Por construcción $\varphi$ es compatible con la trivialización formal $\phi$ en el sentido de la definición 4.1 .

Este resultado nos permite demostrar el siguiente:

Teorema 4.16. El functor $\mathcal{H}_{\text {iggs }}^{\infty}$ es representable por un subesquema cerrado de $\mathcal{U}_{X}^{\infty} \times \mathbb{A}$.

Demostración. Por el Teorema 4.15, la imagen del morfismo:

$$
\mathcal{H}_{i g g s}^{\infty} \hookrightarrow \mathcal{U}_{X}^{\infty} \times \mathbb{A}
$$

está formada por los $(W, p(T))$ tales que $\left.T\right|_{W}(W) \subseteq W \cdot \Omega$. Por la Proposición 4.12 tenemos que $W \cdot \Omega \in \operatorname{Gr}(V)$. El resultado se sigue entonces del Lema 3.21 . 


\subsection{Construcción espectral y la Grassmanniana infinita relativa.}

El objetivo de este apartado es dar una definición del morfismo de Krichever en términos de los datos espectrales asociados a un par de Higgs, es decir, en términos de la curva espectral y del haz de línea sobre la misma que define el fibrado. La clave está en ver que dado un punto $(E, \varphi, \phi)$ de $\operatorname{Higgs}_{X}^{\infty}$, podemos elegir una trivialización formal de $E$ pensado como haz de línea sobre la curva espectral.

Definición 4.17. Llamaremos curva formal base a Spf $k[[z]]$. Dado $p(T) \in \mathbb{A}(k)$, se define la curva espectral formal sobre $k$ como:

$$
\operatorname{Spf} k[[z]][T] / p(T) .
$$

Sea $(E, \phi, \varphi)$ un punto de $\mathcal{H}_{i g g s_{X}^{\infty}}$ con valores en un $k$-esquema $S$. Recordemos que el morfismo de Hitchin formal (4.8):

$$
\mathcal{H}_{\infty}: \mathcal{H i g g s}_{X}^{\infty} \rightarrow \mathbb{A}
$$

asocia a $(E, \phi, \varphi)$ el polinomio característico $p_{\widehat{\varphi}}(T) \in \mathbb{A}(S)$ del campo de Higgs formal $\widehat{\varphi}$.

Proposición 4.18. Sea $S$ un k-esquema. Dado $(E, \phi, \varphi) \in \mathcal{H i g g s}_{X}^{\infty}(S)$, existe una trivialización formal de $E$ a lo largo de $x \times S$ :

$$
\widehat{E}_{x \times S} \simeq \mathcal{O}_{S}[[z]][T] / p_{\bar{\varphi}}(T)
$$

de modo que la matriz $M$ de la Definición 4.1 corresponde a la multiplicación por $T$ en $\mathcal{O}_{S}[[z]][T] / p_{\bar{\varphi}}(T)$ respecto de la base $\left\{1, T, \ldots, T^{n-1}\right\}$.

Demostración. La demostración usa la correspondencia clásica entre pares de Higgs y haces de línea en la curva espectral (véase [7] o la sección 4.1). El campo de Higgs formal $\bar{\varphi}$ dota a $\widehat{E}_{x \times S}$ de una estructura de haz de línea sobre la curva espectral formal $\operatorname{Spf} \mathcal{O}_{S}[[z]][T] / p_{\bar{\varphi}}(T)$. Podemos entonces elegir una trivialización:

$$
\widehat{E}_{x \times S} \simeq \mathcal{O}_{S}[[z]][T] / p_{\bar{\varphi}}(T) .
$$

Usando la trivialización formal:

$$
\phi: \widehat{E}_{x \times S} \sim \mathcal{O}_{S}[[z]]^{n}
$$

podemos definir una estructura de $\mathcal{O}_{S}[[z]]$-álgebra en $\mathcal{O}_{S}[[z]]^{n}$ y, de la condición de compatibilidad de $\phi$ y $\varphi$ de la Definición 4.1, se deduce que la matriz $M$ corresponde a la multiplicación por $T$ en $\mathcal{O}_{S}[[z]][T] / p_{\bar{\varphi}}(T)$.

Acabamos de ver que es posible elegir una trivialización formal de $E$ pensado como haz de línea sobre la curva espectral, en otras palabras, hemos visto directamente que existe una trivialización formal (en el caso de puntos racionales):

$$
\widehat{E}_{x} \simeq\left(\widehat{\pi_{*} \mathcal{O}_{X_{\varphi}}}\right)_{x} \simeq k[[z]][T] / p_{\bar{\varphi}}(T)
$$

donde $\mathcal{O}_{X_{\varphi}}$ denota el anillo de la curva espectral $\pi: X_{\varphi} \rightarrow X$. 


\subsubsection{Grassmanniana infinita relativa.}

Observemos que para cada $p(T) \in \mathbb{A}(S)$ el $\mathcal{O}_{S}((z))$-módulo $V_{S}=\mathcal{O}_{S}((z))^{n}$ puede enriquecerse con una estructura de $\mathcal{O}_{S}((z))$-álgebra:

$$
V_{S} \simeq \mathcal{O}_{S}((z))[T] / p(T) .
$$

Tomemos ahora un punto racional $(E, \phi, \varphi) \in \mathcal{H i g g s}_{X}^{\infty}(k)$ y denotemos por $\pi: X_{\varphi} \rightarrow$ $X$ al revestimiento espectral.

Usando la trivialización formal de la Proposición 4.18

$$
\phi_{\varphi}: \widehat{E}_{x} \simeq k[[z]][T] / p_{\widehat{\varphi}}(T)
$$

es fácil comprobar que:

$$
W=\phi_{\varphi} \mathrm{H}^{0}\left(X_{\varphi}-\pi^{-1}(x), E\right)
$$

es un punto racional de la Grassmanniana infinita $\operatorname{Gr}\left(k((z))[T] / p_{\widehat{\varphi}}(T)\right)$.

El objetivo ahora es dejar variar el polinomio $p(T)$, lo que nos llevará a definir una Grassmanniana infinita relativa. Dado que la construcción es similar a la de la Grassmanniana infinita definida en [3, Sección 2] (véase también el Apéndice A), nos permitiremos la licencia de omitir las demostraciones y dirigimos al lector interesado a consultar [36]).

Sea $p^{\text {univ }}(T)$ al polinomio universal de $\mathbb{A}$, esto es, el punto que corresponde al morfismo identidad Id $: \mathbb{A} \rightarrow \mathbb{A}$ (recordemos que la Proposición 4.6 nos dice que $\mathbb{A}$ es representable). Pensaremos $\mathcal{O}_{\mathbb{A}}[[z]][T] / p^{\text {univ }}(T)$ como el anillo de la curva espectral formal universal.

Denotemos:

$$
V=\mathcal{O}_{\mathbb{A}}((z))[T] / p^{u n i v}(T) \quad V^{+}=\mathcal{O}_{\mathbb{A}}[[z]][T] / p^{u n i v}(T)
$$

como haces de $\mathcal{O}_{\mathbb{A}}$-módulos.

Existe una noción de conmensurabilidad para sub- $\mathcal{O}_{\mathbb{A}}$-módulos de $V$ y verifica propiedades equivalentes a aquellas de la conmensurabilidad estandar.

Definición 4.19. Definimos el functor Grassmanniana infinita relativa para la terna $\left(V, V^{+}, V_{1}\right)$ como aquel que asocia a cada $\mathbb{A}$-esquema $S$ el conjunto:

$$
\mathcal{G r}(V)(S):=\left\{\begin{array}{c}
\text { sub- } \mathcal{O}_{S} \text {-módulos cuasicoherentes } W \subseteq V_{S} \text { t.q. } V_{S} / W \text { es } \\
\text { plano y para cada punto de } S \text { existe un entorno } U \text { y } \\
C \text { conmensurable con } V^{+} \text {tal que } V_{U} /\left(W_{U}+C_{U}\right)=0 \\
\text { y } W_{U} \cap C_{U} \text { es loc. libre de tipo finito }
\end{array}\right\}
$$

Teorema 4.20. El functor $\mathcal{G} \mathrm{r}(V)$ es representable por un $\mathbb{A}$-esquema que seguiremos denotando $\mathcal{G} \mathrm{r}(V)$. En particular es representable por un $k$-esquema. 
Teorema 4.21. Para todo $\mathbb{A}$-esquema $S$ escribamos $V_{S}=V \widehat{\otimes}_{\mathcal{O}_{\mathbb{A}}} \mathcal{O}_{S}$. Se verifica que:

$$
\mathcal{G r}\left(V_{S}\right) \simeq \mathcal{G r}(V) \times_{\mathbb{A}} S .
$$

En particular, para cada punto racional $p(T): k \rightarrow \mathbb{A}$ se tiene que:

$$
\mathcal{G} \mathrm{r}\left(V_{k}\right)=\mathcal{G r}(k((z))[T] / p(T))
$$

Esto significa además que:

$$
\mathcal{G r}\left(\mathcal{O}_{\mathbb{A}}((z))[T] / p^{u n i v}(T)\right) \rightarrow \mathbb{A}
$$

es una fibración de Grassmannianas infinitas.

Observación 4.22. Observemos que se tiene un isomorfismo de $\mathcal{O}_{\mathbb{A}}$-módulos:

$$
\mathcal{O}_{\mathbb{A}}((z))[T] / p^{\text {univ }}(T) \simeq \mathcal{O}_{\mathbb{A}}((z))^{n},
$$

luego si consideramos la fibración trivial de $k$-esquemas:

$$
\operatorname{Gr}\left(k((z))^{n}\right) \times \mathbb{A} \rightarrow \mathbb{A}
$$

se tienen isomorfismos de $\mathbb{A}$-esquemas:

$$
\operatorname{Gr}\left(k((z))^{n}\right) \times \mathbb{A} \simeq \mathcal{G r}\left(\mathcal{O}_{\mathbb{A}}((z))^{n}\right) \simeq \mathcal{G} \mathrm{r}\left(\mathcal{O}_{\mathbb{A}}((z))[T] / p^{\text {univ }}(T)\right)
$$

\subsubsection{Morfismo de Krichever vía la construcción espectral.}

Una vez construida la Grassmanniana infinita relativa, estamos en condiciones de dar un morfismo de Krichever que haga uso de ella. Utilizamos la construcción espectral para definirlo y, en virtud del isomorfismo anterior 4.3.1, resulta además que es equivalente al ya dado.

Dado un punto $(E, \phi, \varphi) \in \mathcal{H}_{\text {iggs }}^{\infty}(S)$, podemos usar la trivialización formal de $E$ como haz de línea en la curva espectral (véase la Proposición 4.18) y definir un morfismo de Krichever:

$$
\begin{aligned}
\mathcal{H i g g s}_{X}^{\infty}(S) & \rightarrow \mathcal{G} \mathrm{r}\left(\mathcal{O}_{\mathbb{A}}((z))[T] / p^{\text {univ }}(T)\right)(S) \\
(E, \phi, \varphi) & \mapsto \phi_{\varphi}\left(\underset{m}{\lim } \pi_{*}^{\prime} E_{\varphi}(m)\right)
\end{aligned}
$$

donde $\pi^{\prime}: X_{\varphi} \rightarrow S$ y $E_{\varphi}$ es el fibrado $E$ pensado como haz de línea en la curva espectral. Para puntos racionales vale:

$$
\begin{aligned}
\mathcal{H i g g s}_{X}^{\infty}(k) & \rightarrow \mathcal{G r}\left(\mathcal{O}_{\mathbb{A}}((z))[T] / p^{\text {univ }}(T)\right)(k) \\
(E, \phi, \varphi) & \mapsto \phi_{\varphi} \mathrm{H}^{0}\left(X_{\varphi}-\pi^{-1}(p), E_{\varphi}\right)
\end{aligned}
$$

siendo $\pi: X_{\varphi} \rightarrow X$ el revestimiento espectral. Teniendo en cuenta el isomorfismo 4.3.1):

$$
\operatorname{Gr}\left(k((z))^{n}\right) \times \mathbb{A} \simeq \mathcal{G} \mathrm{r}\left(\mathcal{O}_{\mathbb{A}}((z))[T] / p^{\text {univ }}(T)\right)
$$

se sigue que este morfismo de Krichever es en realidad equivalente al morfismo de Krichever de la Definición 4.9, y por el Teorema 4.10 es inyectivo. 


\section{Capítulo 5}

\section{Moduli de haces de línea en revestimientos y sistemas integrables.}

Este capítulo consta de 4 secciones. En las dos primeras construiremos el espacio de moduli de revestimientos y el espacio de moduli de haces de línea sobre revestimientos, y demostraremos que son subesquemas de la Grassmanniana relativa. Utilizaremos estos espacios para las dos últimas secciones, en las que demostramos la existencia de un sistema integrable con propiedades análogas a las del sistema de Hitchin, y hacemos un estudio sobre fibrados sobre una curva $X$ que son imagen directa de un haz de línea sobre un revestimiento finito de $X$, encontrando así una relación con el Programa de Abelianización de Hitchin.

\subsection{Moduli de revestimientos de grado $n$.}

Estudiamos en esta sección el moduli de revestimientos $\pi: Y \rightarrow X$ punteados de curvas lisas e íntegras con parámetros formales. Nuestro punto de vista será distinto al dado en [31, 32], pues no fijaremos una compatibilidad entre los parámetros formales en el punto marcado $x$ de $X$ y los parámetros formales en los puntos de $\pi^{-1}(x)$. Es precisamente este hecho, el hecho de permitir variar la estructura local de revestimiento, lo que nos obligará a definir el morfismo de Krichever valorando en una Grassmanniana relativa (como pasaba con los pares de Higgs del capítulo anterior). Utilizando las ideas de [32, 31] demostraremos que este espacio de moduli es representable por un subesquema dentro la Grassmanniana relativa.

Restringimos el estudio a los siguientes tipos de revestimientos. Sea $\pi: Y \rightarrow X$ un morfismo finito de grado $n$ y separable de curvas propias y lisas sobre un cuerpo algebraicamente cerrado $k$, donde $Y$ es reducida y $X$ es íntegra. Sea $x \in X$ un punto liso. Definimos $A:=H^{0}\left(X-x, \mathcal{O}_{X}\right), B:=H^{0}\left(Y-\pi^{-1}(x), \mathcal{O}_{Y}\right), \Sigma_{X}$ el cuerpo de funciones de $A$ y $\Sigma_{Y}$ el anillo total de cocientes de $B$. Denotemos por Tr la traza de $\Sigma_{Y}$ como $\Sigma_{X}$-álgebra finita.

Diremos que la terna $(Y, X, x)$ tiene la propiedad $(*)$ cuando $\operatorname{Tr}(B) \subseteq A$. 
Observación 5.1. Si $\pi$ es un morfismo plano, entonces $\pi_{*} \mathcal{O}_{Y}$ es un $\mathcal{O}_{X}$-módulo localmente libre (y de tipo finito por ser $\pi$ finito), y por tanto $B$ es un $A$-módulo localmente libre de tipo finito. Sean $U_{i}$ abiertos de Spec $A$ tales que $B_{U_{i}}$ es libre sobre $A_{U_{i}}$, si $b \in B_{U_{i}}$, tomando una base de $B_{U_{i}}$ sobre $A_{U_{i}}$ y denotando por $M_{b}$ a la matriz del endomorfismo multiplicar por $b$ en esa base, se sigue la traza de $b$ es el coeficiente de grado $m-1$ del polinomio característico $\operatorname{det}\left(x I-M_{b}\right)$ de grado $m$, que está en $A_{U_{i}}$. Como la traza es estable por cambios de base planos y localizar lo es, se tiene que las trazas $\operatorname{Tr}_{i}$ y $\operatorname{Tr}_{j}\left(\operatorname{Tr}_{i}: B_{U_{i}} \rightarrow A_{U_{i}}\right)$ coinciden en $U_{i} \cap U_{j}$, con lo pegan y dan una traza $\operatorname{Tr}: B \rightarrow A$ tal que $\operatorname{Tr}_{\mid U_{i}}=\operatorname{Tr}_{i}$. Luego $\operatorname{Tr}(B) \subseteq A$.

Observación 5.2. Si $X$ es una curva íntegra y lisa entonces $\pi$ es plano, luego por la observación anterior también es $\operatorname{Tr}(B) \subseteq A$. En efecto, por ser $Y$ reducida se deduce que los puntos asociados a $Y$ (aquellos tales que todo elemento del maximal del anillo local del punto que definen es un divisor del cero) son precisamente los puntos genéricos de sus componentes irreducibles $Y_{i}$. Además, como $\pi$ es un morfismo finito se tiene que $\pi_{i}: Y_{i} \rightarrow X$ son finitos (y por tanto cerrados), luego si $\pi_{i}\left(Y_{i}\right) \neq X$ entonces sería un conjunto finito de puntos $\left\{x_{1}, \ldots, x_{h}\right\}$ y por tanto:

$$
Y_{i}=\pi_{i}^{-1}\left(x_{1}\right) \cup \cdots \cup \pi_{i}^{-1}\left(x_{h}\right)
$$

Como $Y_{i}$ es irreducible tiene que ser $h=1$, lo que es una contradicción con que $\pi_{i}$ sea finito. Es decir, toda componente irreducible de $Y$ domina a $X$ (en el sentido de que su imagen es densa) y por tanto los puntos genéricos de las componentes irreducibles van a parar al punto genérico de $X$ por $\pi$. Se sigue entonces que $\pi$ es plano ([19, III, 9.7]).

Proposición 5.3. Sea $\pi: Y \rightarrow X$ revestimiento separable de grado n entre curvas lisas, integras y propias sobre $k$. Sea $x \in X$ un punto tal que $\pi^{-1}(x)=\sum_{i=1}^{r} n_{i} y_{i}$ con $y_{i} \in Y$, $y_{i} \neq y_{j}$ para todo $i \neq j, y \sum_{i=1}^{r} n_{i}=n$. Entonces:

- Existe un parámetro formal de $X$ en $x$, i.e., existe un isomorfismo:

$$
z_{x}: \widehat{\mathcal{O}}_{X, x} \simeq k[[z]]
$$

- Sea $\bar{y}=\pi^{-1}(x)=\sum_{i=1}^{r} n_{i} y_{i}$ con $\sum_{i=1}^{r} n_{i}=n$. Para cada $y_{i} \in \bar{y}$, existe un parámetro formal de $Y$ en $y_{i}$ :

$$
z_{y_{i}}: \widehat{\mathcal{O}}_{Y, y_{i}} \simeq k\left[\left[z_{i}\right]\right]
$$

- Dadas $z_{x}$ y $\left\{z_{y_{i}}\right\}_{i=1}^{r}$ existen, de modo canónico, polinomios mónicos, irreducibles de grado $n_{i}, p_{i}(T)$, con coeficientes en $k[[z]]$, tales que el siguiente diagrama es un diagrama conmutativo de $k$-álgebras:

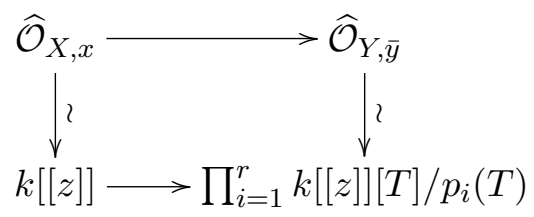


Demostración. Localizando el morfismo $\mathcal{O}_{X} \rightarrow \mathcal{O}_{Y}$ en el punto $x$ se tiene un morfismo:

$$
\mathcal{O}_{X, x} \rightarrow \mathcal{O}_{Y, x}
$$

y como la fibra de $x$ es un conjunto finito $\left\{y_{1}, \ldots, y_{r}\right\}$ de puntos no singulares de $Y$ (que forman el espectro maximal de $\mathcal{O}_{Y, x}$ ) se tiene que $\mathcal{O}_{Y, x}$ es un anillo de Dedekind y por lo tanto se tiene una descomposición primaria:

$$
\mathfrak{m}_{x} \mathcal{O}_{Y, x}=\mathfrak{m}_{1}^{n_{1}} \cdots \cdots \mathfrak{m}_{r}^{n_{r}}
$$

de modo que el anillo de la fibra vale:

$$
\mathcal{O}_{Y, x} / \mathfrak{m}_{x} \mathcal{O}_{Y, x}=\oplus_{i=1}^{r} \mathcal{O}_{Y, \mathfrak{m}_{i}} / \mathfrak{m}_{i}^{n_{i}}
$$

Por lo tanto, los maximales $\mathfrak{m}_{i}$ son los maximales $\mathfrak{m}_{y_{i}}$ de los puntos $y_{i}$ en la fibra de $x$ y se tiene:

$$
\mathfrak{m}_{x} \mathcal{O}_{Y, y_{i}}=\mathfrak{m}_{y_{i}}^{n_{i}}
$$

Es fácil entonces ver que:

$$
\widehat{\mathcal{O}}_{Y, x} \simeq \oplus_{i=1}^{r} \widehat{\mathcal{O}}_{Y, y_{i}}
$$

Como $\widehat{\mathcal{O}}_{X, x}$ y $\widehat{\mathcal{O}}_{Y, y_{i}}$ son $k$-álgebras locales, regulares y completas de dimensión uno, por el teorema de Cohen se sigue que:

$$
\widehat{\mathcal{O}}_{X, x} \simeq k[[z]], \quad \widehat{\mathcal{O}}_{Y, y_{i}} \simeq k\left[\left[z_{i}\right]\right] .
$$

A través del morfismo de $k$-álgebras:

$$
k[[z]] \stackrel{z_{x}^{-1}}{\longrightarrow} \widehat{\mathcal{O}}_{X, x} \rightarrow \widehat{\mathcal{O}}_{Y, y_{i}} \stackrel{z_{y_{i}}}{\longrightarrow} k\left[\left[z_{i}\right]\right],
$$

se deduce que $k\left[\left[z_{i}\right]\right]$ es una extensión irreducible y separable de $k[[z]]$ de grado $n_{i}$, luego $z_{i}$ verifica un polinomio mínimo, $p_{i}(T)$, con coeficientes en $k[[z]]$ de grado $n_{i}$, es decir, tenemos un isomorfismo de $k[[z]]$-álgebras:

$$
k\left[\left[z_{i}\right]\right] \simeq k[[z]][T] / p_{i}(T)
$$

Observación 5.4. Observemos además que podemos escoger:

$$
p_{i}(T)=T^{n_{i}}-z u_{i}(z, T),
$$

donde $u_{i}(z, T) \in k[[z]][T]$ es de grado menor que $n_{i}$ y $u_{i}(0,0)=1$. En efecto, se tiene que $\mathfrak{m}_{x} \mathcal{O}_{Y, y_{i}}=\mathfrak{m}_{i}^{n_{i}}$ y por el teorema de Cohen $\widehat{\mathcal{O}}_{Y, y_{i}} \simeq k\left[\left[z_{i}\right]\right]$. Por lo tanto, en $\widehat{\mathcal{O}}_{Y, y_{i}}$ se tiene:

$$
z=z_{i}^{n_{i}} v_{i}\left(z_{i}\right)
$$


siendo $v_{i}\left(z_{i}\right)$ un elemento invertible en $\widehat{\mathcal{O}}_{Y, y_{i}}$, es decir:

$$
v_{i}\left(z_{i}\right)=v_{i, 0}+v_{i, 1} z_{i}+v_{i, 2} z_{i}^{2}+\cdots \quad \text { con } \quad v_{i, 0} \neq 0 .
$$

Como $k$ es algebraicamente cerrado de característica nula, podemos hacer el cambio $z_{i}=$ $v_{i, 0}^{1 / n_{i}} z_{i}$, y por tanto suponer directamente que $v_{i, 0}=1$. Por otro lado, $k[[z]] \rightarrow k\left[\left[z_{i}\right]\right]$ es un morfismo entero, luego se tiene la siguiente relación:

$$
T^{n_{i}}+b_{1}^{(i)} T^{n_{i}-1}+\cdots+b_{n_{i}}^{(i)}=0
$$

donde $b_{j}^{(i)} \in k[[z]]$. Es decir:

$$
k\left[\left[z_{i}\right]\right] \simeq k[[z]][T] / T^{n_{i}}+b_{1}^{(i)} T^{n_{i}-1}+\cdots+b_{n_{i}}^{(i)}
$$

donde además, si hacemos $z=0$ hemos de obtener un punto contado $n_{i}$ veces, lo que significa que $b_{j}^{(i)}=z \bar{b}_{j}^{(i)} \operatorname{con} \bar{b}_{j}^{(i)} \in k[[z]]$. De lo que resulta:

$$
T^{n_{i}}=-z\left(\bar{b}_{1}^{(i)} T^{n_{i}-1}+\cdots+\bar{b}_{n_{i}}^{(i)}\right) .
$$

Si denotamos entonces $u_{i}(z, T)$ a $v_{i}(z, T)^{-1}$, se tiene que $u_{i}(z, T)$ es en realidad un polinomio en $T$ con coeficientes en $k[[z]]$ tal que $u_{i}(0,0)=1 \mathrm{y}$ :

$$
k\left[\left[z_{i}\right]\right] \simeq k[[z]][T] / T^{n_{i}}-z u_{i}(z, T) .
$$

Observación 5.5. Si en vez de $k$ tomamos directamente un esquema $S$, el cambio $z_{i}=$ $v_{i, 0}^{1 / n_{i}} z_{i}$ no puede hacerse a menos que existan raíces $n_{i}$-ésimas en $\mathcal{O}_{S}$. Este problema se arregla dotando a la categoría de $S$-esquemas de la topología étale.

Definición 5.6. Definimos el functor de revestimientos con índices de ramificación $\underline{n}=$ $\left(n_{1}, \ldots, n_{r}\right), \mathcal{C}_{0} v_{\underline{n}}^{\infty}$, como el hacificado del functor contravariante sobre la categoría de $k$ esquemas que asigna, a cada $k$-esquema $S$ el conjunto de clases de equivalencia de los datos $\left(Y, X, \pi, x, \bar{y}, z_{x}, z_{\bar{y}}\right)$, donde:

1. $p_{Y}: Y \rightarrow S$ es un morfismo propio y plano cuyas fibras son curvas geométricamente lisas e íntegras.

2. $p_{X}: X \rightarrow S$ es un morfismo propio y plano cuyas fibras son curvas geométricamente lisas e íntegras.

3. $\pi: Y \rightarrow X$ es un morfismo finito y separable de $S$-esquemas de grado $n$ tal que sus fibras sobre los puntos cerrados de $S$ verifican (*).

4. $x: S \rightarrow X$ es una sección de $p_{X}$.

5. $\bar{y}=\left\{y_{1}, \ldots, y_{r}\right\}$ es un conjunto de secciones disjuntas de $p_{Y}$ tales que:

$$
\pi^{-1}(x(S))=n_{1} y_{1}(S)+\cdots+n_{r} y_{r}(S) .
$$


6. $z_{x}$ es un parámetro formal a lo largo de $x(S)$ :

$$
z_{x}: \widehat{\mathcal{O}}_{X, x(S)} \simeq \mathcal{O}_{S}[[z]]
$$

7. $z_{\bar{y}}=\left\{z_{y_{i}}\right\}$ es una trivialización formal en $\bar{y}$ :

$$
z_{\bar{y}}: \widehat{\mathcal{O}}_{Y, \bar{y}(S)} \simeq \mathcal{O}_{S}\left[\left[z_{1}\right]\right] \times \cdots \times \mathcal{O}_{S}\left[\left[z_{r}\right]\right]
$$

de modo que para cada $y_{i}(S) \in \bar{y}(S), z_{y_{i}}$ es un parámetro formal de $Y$ a lo largo de $y_{i}(S)$ :

$$
z_{y_{i}}: \widehat{\mathcal{O}}_{Y, y_{i}(S)} \simeq \mathcal{O}_{S}\left[\left[z_{i}\right]\right] .
$$

8. $\left(Y, X, \pi, x, \bar{y}, z_{x}, z_{\bar{y}}\right)$ y $\left(Y^{\prime}, X^{\prime}, \pi^{\prime}, x^{\prime}, \bar{y}^{\prime}, z_{x^{\prime}}, z_{\bar{y}^{\prime}}\right)$ se dice que son equivalentes cuando exista un diagrama de $S$-esquemas:

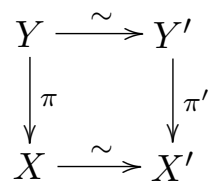

compatible con todos los datos. Para abreviar, denotaremos $\mathcal{Y}$ a sus puntos.

Observación 5.7. La diferencia que existe entre esta definición y la definición del functor de Hurwitz ([31, Definition 4]), es que nosotros no fijamos una compatibilidad entre los parámetros formales $z$ y $z_{i}$, mientras que en [31] se fija la relación: $z_{i}^{n_{i}}=z$. Es decir, el polinomio en su caso es fijo y se escribe $p_{i}(T)=T^{n_{i}}-z$ y en nuestro caso permitimos que varíe.

En lo que sigue escribiremos $V_{\underline{n}}=k\left(\left(z_{1}\right)\right) \times \cdots \times k\left(\left(z_{r}\right)\right)$.

Como en [31] definimos el morfismo de Krichever:

Definición 5.8. Se define el morfismo de Krichever por:

$$
\begin{aligned}
\operatorname{Kr}(S): \mathcal{C}_{o v}^{\infty}(S) & \rightarrow \operatorname{Gr}\left(V_{\underline{n}}\right)(S) \\
\mathcal{Y} & \mapsto z_{\bar{y}} \underset{\vec{m}}{\lim _{Y, *} p_{Y}(m \bar{y})}
\end{aligned}
$$

Para puntos racionales vale:

$$
\begin{aligned}
& \operatorname{Kr}(k): \operatorname{Cov}_{\underline{n}}^{\infty}(k) \rightarrow \operatorname{Gr}\left(V_{\underline{n}}\right)(k) \\
& \mathcal{Y} \mapsto z_{\bar{y}} \mathrm{H}^{0}\left(Y-\bar{y}, \mathcal{O}_{Y}\right)
\end{aligned}
$$

Definición 5.9. Dada una partición $\underline{n}=\left(n_{1}, \ldots, n_{r}\right)$, definimos:

$$
\mathbb{A}_{\underline{n}}:=\mathbb{A}_{n_{1}} \oplus \cdots \oplus \mathbb{A}_{n_{r}},
$$


donde $\mathbb{A}_{n_{i}}$ es el $k$-esquema que representa al functor:

$$
S \rightsquigarrow \mathcal{O}_{S}[[z]]^{n_{i}}
$$

y cuyos puntos interpretaremos como polinomios mónicos, irreducibles y separables de grado $n_{i}$ con coeficientes en $\mathcal{O}_{S}[[z]]$ del tipo de la Observación 5.4 (véanse la Proposición 4.6 y la Observación 4.7 . $\mathbb{A}_{\underline{n}}$ es un subesquema abierto de $\mathbb{A}$.

Gracias a la Proposición 5.3 podemos definir un morfismo:

$$
\begin{aligned}
C: \mathcal{C}_{\mathcal{O} v_{\underline{n}}}^{\infty} & \rightarrow \mathbb{A}_{\underline{n}} \\
\mathcal{Y} & \mapsto \underline{p}(T)=\left(p_{1}(T), \ldots, p_{r}(T)\right)
\end{aligned}
$$

y por lo tanto tenemos un morfismo de Krichever:

$$
\begin{aligned}
\mathcal{C o v}_{\underline{\underline{n}}}^{\infty}(S) & \rightarrow \operatorname{Gr}\left(V_{\underline{n}}\right)(S) \times \mathbb{A}_{\underline{n}}(S) \\
\mathcal{Y} & \mapsto\left(z_{\bar{y}} \underset{m}{\lim _{m}} p_{Y, *} \mathcal{O}_{Y}(m \bar{y}), \underline{p}(T)\right)
\end{aligned}
$$

que nos permitirá además controlar la variación del polinomio $p(T)$, la estructura local de revestimiento.

Denotaremos $V_{\underline{n}}^{\text {univ }}$ al producto $\prod_{i=1}^{r} \mathcal{O}_{\mathbb{A}_{n_{i}}}((z))[T] / p_{i}^{\text {univ }}(T)$ con la siguiente estructura de $\mathcal{O}_{\mathbb{A}_{\underline{\underline{n}}}}((z))$-álgebra:

$$
\begin{aligned}
\mathcal{O}_{\mathbb{A}_{\underline{n}}}((z)) & \rightarrow \mathcal{O}_{\mathbb{A}_{n_{1}}}((z))[T] / p_{1}^{\text {univ }}(T) \times \cdots \times \mathcal{O}_{\mathbb{A}_{n_{r}}}((z))[T] / p_{r}^{\text {univ }}(T) \\
z & \mapsto\left(T^{n_{1}} u_{1}(z, T)^{-1}, \ldots, T^{n_{r}} u_{r}(z, T)^{-1}\right)
\end{aligned}
$$

donde $p_{i}^{\text {univ }}(T)$ son los polinomios universales de $\mathbb{A}_{n_{i}}$ (correspondientes al punto Id: $\mathbb{A}_{n_{i}} \rightarrow$ $\left.\mathbb{A}_{n_{i}}\right)$, y que escribimos $p_{i}^{\text {univ }}(T)=T^{n_{i}}-z u_{i}(z, T)$, con $u_{i}(z, T) \in \mathcal{O}_{\mathbb{A}_{n_{i}}}[[z]][T]$.

Teniendo en cuenta el isomorfismo de la ecuación 4.3.1, puede verse que:

$$
\operatorname{Gr}\left(V_{\underline{n}}\right) \times \mathbb{A}_{\underline{n}} \simeq \mathcal{G} \mathrm{r}\left(V_{\underline{n}}^{\text {univ }}\right),
$$

siendo $\mathcal{G} \mathrm{r}\left(V_{\underline{n}}^{\text {univ }}\right)$ la Grassmanniana infinita relativa definida en 4.19 . De este modo, podemos reinterpretar el morfismo de Krichever de la ecuación (5.1.3), definiendo su versión relativa:

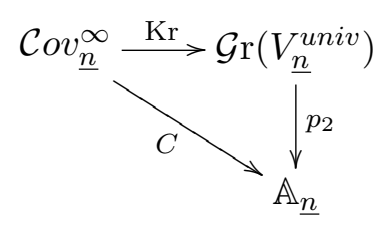

donde $C(\mathcal{Y})=\underline{p}(T)$ y, en virtud del isomorfismo 5.1.5, $p_{2}$ es la proyección en el segundo factor. 
Observación 5.10. Nótese que la fibra de $C$ en el punto racional:

$$
\underline{p}(T)=\left(T^{n_{1}}-z, \ldots, T^{n_{r}}-z\right)
$$

de $\mathbb{A}_{\underline{n}}$, es precisamente el moduli revestimientos punteados con trivialización formal tratado en [31]; las fibras de $p_{2}$ en dicho punto son Grassmannianas del tipo:

$$
\operatorname{Gr}\left(k\left(\left(z_{1}\right)\right) \times \cdots \times k\left(\left(z_{r}\right)\right)\right)
$$

donde la estructura de $k((z))$-álgebra de $k\left(\left(z_{1}\right)\right) \times \cdots \times k\left(\left(z_{r}\right)\right)$ viene dada por $z \mapsto$ $\left(z_{1}^{n_{1}}, \ldots, z_{r}^{n_{r}}\right)$, y el morfismo inducido por $\mathrm{Kr}$ entre las fibras es el morfismo de Krichever definido en [31].

El estudio recogido en [31] nos va a permitir caracterizar la imagen de este morfismo de Krichever relativo. Para cada $\mathbb{A}_{\underline{n}}$-esquema $S$, denotamos:

$$
V_{S}=V_{\underline{n}}^{u n i v} \hat{\otimes}_{\mathcal{O}_{\mathbb{A}_{\underline{n}}}} \mathcal{O}_{S} .
$$

Sea $\operatorname{Tr}: V_{S} \rightarrow \mathcal{O}_{S}((z))$ el morfismo traza, que es una aplicación $\mathcal{O}_{S}((z))$-lineal. Para cada sub- $\mathcal{O}_{S}$-álgebra $B$ de $V_{S}$ escribiremos $\operatorname{Tr}(B) \subseteq \mathcal{O}_{S}((z))$ como la imagen de $B$ vía la

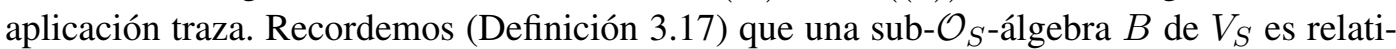
vamente regular sobre $S$, cuando para cada punto cerrado $s \in S$ se verifique que el cambio de base $B_{s}$ de $B$ a $s \in S$ sea un anillo regular (condición que geométricamente se traducirá en la lisitud de las fibras de la familia de curvas).

Teorema 5.11. Un punto $B \in \mathcal{G} r\left(V_{n}^{u n i v}\right)(S)$ está en la imagen del morfismo de Krichever

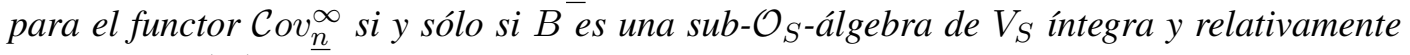
regular, $y \operatorname{Tr}(B) \subseteq \bar{B}$. En particular, el morfismo de Krichever es inyectivo.

\section{Demostración.}

$\Rightarrow$ ) Dado que $\mathcal{G r}\left(V_{n}^{\text {univ }}\right)$ es un haz, podemos suponer que $S=\operatorname{Spec} \mathcal{O}$ es el espectro de un anillo local. Sea $\mathcal{Y} \in \mathcal{C}_{o v} v_{n}^{\infty}$ tal que $B=\operatorname{Kr}(\mathcal{Y})$. Por la propia definición del morfismo de Krichever se tienen las siguientes propiedades:

$$
\mathcal{O} \subset B, \quad B \cdot B \subseteq B, \quad A \subseteq B \cap \mathcal{O}((z)) \subseteq \operatorname{Tr}(B),
$$

siendo $A=\operatorname{Kr}\left(X, x, z_{x}\right)$. La estabilidad por cambio de base del morfismo traza, y la condición 3 de la Definición 5.6 nos dicen que, para cada punto cerrado $s \in S$, se verifica:

$$
A_{s}=B_{s} \cap \kappa(s)((z))=\operatorname{Tr}\left(B_{s}\right)=\operatorname{Tr}(B)_{s},
$$

luego $(\operatorname{Tr}(B) / A)_{s}=0$. Como $B$ es un $A$-módulo finito, $\operatorname{Tr}(B)$ también lo es (pues es un cociente suyo) y por lo tanto $\operatorname{Tr}(B) / A$ es finito. Aplicando Nakayama se sigue que $\operatorname{Tr}(B) / A=0$. De donde se deduce:

$$
A=B \cap \mathcal{O}((z))=\operatorname{Tr}(B),
$$


lo que implica $\operatorname{Tr}(B) \subseteq B$.

$\Leftarrow) \mathrm{Si} B \in \mathcal{G} \mathrm{r}\left(V_{n}^{\text {univ }}\right)(S)$ es una sub-O $\mathcal{O}$-álgebra de $V_{S}$, entonces podemos recuperar la terna $\left(Y, \bar{y}, z_{\bar{y}}\right)$ (véase el Apédice B. El Lema 4.4 de [31] demuestra que si $B \in \mathcal{G} \mathrm{r}\left(V_{\underline{n}}^{\text {univ }}\right)$ $\mathrm{y} \operatorname{Tr}(B) \subseteq B$, entonces:

$$
\operatorname{Tr}(B)=B \cap \mathcal{O}((z)) \in \operatorname{Gr}(k((z)))(S) .
$$

En particular, $\operatorname{Tr}(B)$ es una sub-O-Ólgebra de $\mathcal{O}((z))$, por lo que usando de nuevo la construcción inversa del morfismo de Krichever para curvas (apéndice $B$ recuperamos $\left(X, x, z_{x}\right)$. Finalmente, es fácil comprobar que la inclusión $\operatorname{Tr}(B) \subseteq B$ es compatible con las filtraciones definidas en $\mathcal{O}((z))$ y $V_{S}$ respectivamente, de lo que obtenemos el morfismo $\pi: Y \rightarrow X$. La condición de regularidad se traduce simplemente en la lisitud de las fibras geométricas de la familia de curvas $Y \rightarrow S$. Idem para la condición de anillo íntegro.

Teorema 5.12. El functor $\mathcal{C o v}_{\underline{n}}^{\infty}$ es representable por un subesquema localmente cerrado de la Grassmanniana relativa $\mathcal{G} \mathrm{r}\left(V_{\underline{n}}^{\text {univ }}\right)$.

Demostración. Por el Lema 3.21, las condiciones $\operatorname{Tr}(B) \subset B$ y $B \cdot B \subseteq B$ (condición de ser subálgebra de $V_{S}$ ) son cerradas en $\mathcal{G} \mathrm{r}\left(V_{\underline{n}}^{\text {univ }}\right)$. Los puntos de este cerrado tales que $B$ es relativamente regular forman un abierto.

Observación 5.13. La condición de que $B$ sea una anillo íntegro equivale a que:

$$
B \notin \prod_{i=1}^{r} \mathcal{G} \mathrm{r}\left(V_{n_{i}}^{\text {univ }}\right),
$$

que es un subesquema cerrado de $\mathcal{G} \mathrm{r}\left(V_{\underline{n}}^{\text {univ }}\right)$.

Podemos considerar ahora el morfismo de olvido $\mathcal{C}_{0} v_{\underline{n}}^{\infty} \rightarrow \mathcal{M}^{\infty}$ que manda cada punto de $\mathcal{C}_{o v}^{\infty},\left(Y, X, \pi, x, \bar{y}, z_{x}, z_{y_{i}}\right)$, a la terna $\left(X, x, z_{x}\right) \in \mathcal{M}^{\infty}$. Se tiene entonces que para cada $\left(\bar{X}, x, z_{x}\right) \in \mathcal{M}^{\infty}$, la fibra es precisamente el moduli $\mathcal{C}_{X}{ }_{X, \underline{n}}^{\infty}$ de revestimientos sobre $\left(X, x, z_{x}\right)$ con trivialización formal a lo largo de la fibra de $x$. De modo análogo a la sección anterior se demuestran los siguientes resultados.

Teorema 5.14. Sea $A \in \operatorname{Gr}(k((z)))(S)$ el punto que define la terna $\left(X, x, z_{x}\right) \in \mathcal{M}^{\infty}(S)$ por el morfismo de Krichever ordinario y $B \in \mathcal{G} \mathrm{r}\left(V_{\underline{\underline{n}}}^{\text {univ }}\right)(S)$. B está en la imagen del morfismo de Krichever para $\mathcal{C}_{X, \underline{n}}^{\infty}$ si y sólo si:

- $A \subset B$.

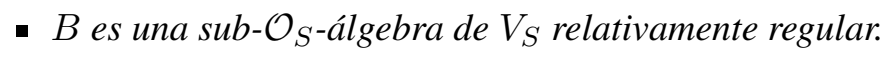

En particular el morfismo de Krichever es inyectivo.

Teorema 5.15. El functor $\operatorname{Cov}_{X, \underline{n}}^{\infty}$ es representable por un subesquema localmente cerrado de la Grassmanniana relativa $\mathcal{G} \mathrm{r}\left(V_{\underline{\underline{n}}}^{\text {univ }}\right)$. 


\subsection{Moduli de haces de línea sobre revestimientos.}

Veamos ahora que el moduli de haces de haces de línea (con trivialización formal) sobre los revestimientos de la sección anterior, es representable por un subesquema de la Grassmanniana relativa.

Definición 5.16. Sea $\tilde{\mathcal{Y}}$ el objeto universal de $\mathcal{C}_{o v}^{\infty}$ (que existe en virtud de 5.12). Definimos el functor $\mathcal{P} i c_{\tilde{\mathcal{Y}}}^{\infty}$ como el hacificado del functor contravariante sobre la categoría de $k$-esquemas que asigna, a cada $k$-esquema $S$ el conjunto de clases de equivalencia de datos $\left(Y, X, \pi, x, \bar{y}, z_{x}, z_{\bar{y}}, L, \phi_{\bar{y}}\right)$, donde:

1. $\left(Y, X, \pi, x, \bar{y}, z_{x}, z_{\bar{y}}\right) \in \mathcal{C}_{\operatorname{Cov}_{\underline{n}}}^{\infty}(S)$. Es decir, si denotamos $f: S \rightarrow \mathcal{C}_{\mathcal{O}_{\underline{n}}}^{\infty}$ al morfismo inducido por $S \rightarrow \mathcal{P} i c_{\tilde{\mathcal{Y}}}^{\infty}$, entonces $f^{*} \tilde{\mathcal{Y}}=\left(Y, X, \pi, x, \bar{y}, z_{x}, z_{\bar{y}}\right)$.

2. $L$ es un haz de línea en $Y$.

3. $\phi_{\bar{y}}=\left\{\phi_{y_{i}}\right\}$ es una familia de trivializaciones formales $L$ a lo largo de $y_{i}$ :

$$
\phi_{y_{i}}: \widehat{L}_{y_{i}} \simeq \widehat{\mathcal{O}}_{Y, y_{i}} .
$$

4. $\left(Y, X, \pi, x, \bar{y}, z_{x}, z_{\bar{y}}, L, \phi_{\bar{y}}\right)$ y $\left(Y^{\prime}, X^{\prime}, \pi^{\prime}, x^{\prime}, \bar{y}^{\prime}, z_{x^{\prime}}, z_{\bar{y}^{\prime}}, L^{\prime}, \phi_{\bar{y}^{\prime}}\right)$ se dice que son equivalentes cuando existan isomorfismos $Y \simeq Y^{\prime}$ y $L \simeq L^{\prime}$ compatibles con todos los datos (véase la definición 3.2). Para abreviar, denotaremos a sus puntos $\mathcal{Y}_{L}$.

Definición 5.17. Se define el morfismo de Krichever:

$$
\begin{aligned}
\operatorname{Kr}(S): \mathcal{P}_{i c_{\tilde{\mathcal{Y}}}^{\infty}}^{\infty}(S) & \rightarrow \mathcal{G} \mathrm{r}\left(V_{\underline{\underline{n}}}^{\text {univ }}\right)(S) \\
\mathcal{Y}_{L} & \mapsto\left(z_{\bar{y}} \circ \phi_{\bar{y}}\right) \underset{\vec{m}}{\lim _{Y, *} L(m \bar{y})}
\end{aligned}
$$

Para puntos racionales:

$$
\begin{aligned}
\operatorname{Kr}(k): \mathcal{P}_{i c_{\tilde{\mathcal{Y}}}^{\infty}}^{\infty}(k) & \rightarrow \mathcal{G} \mathrm{r}\left(V_{\underline{n}}^{\text {univ }}\right)(k) \\
\mathcal{Y}_{L} & \mapsto\left(z_{\bar{y}} \circ \phi_{\bar{y}}\right) \mathrm{H}^{0}(Y-\bar{y}, L)
\end{aligned}
$$

Observación 5.18. Como en la sección anterior, podemos entender el morfismo de Krichever como:

$$
\begin{aligned}
\operatorname{Kr}(S): \mathcal{P}_{i c_{\tilde{\mathcal{Y}}}^{\infty}}^{\infty}(S) & \rightarrow \mathcal{G r}\left(V_{\underline{\underline{n}}}\right)(S) \times \mathbb{A}(S) \\
\mathcal{Y}_{L} & \mapsto\left(\left(z_{\bar{y}} \circ \phi_{\bar{y}}\right) \underset{m}{\lim _{m}} \pi_{Y, *} L(m \bar{y}), p(T)\right)
\end{aligned}
$$

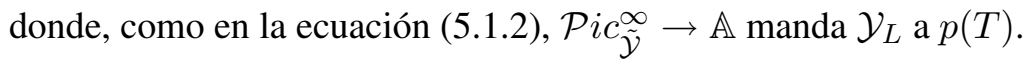

La noción de estabilizador de un punto de la Grassmanniana de la Definición 3.9, se generaliza para este caso como sigue. 
Definición 5.19. Sea $W \in \mathcal{G} r\left(V_{\underline{n}}^{u n i v}\right)(S)$. Se dice que una sub- $\mathcal{O}_{S}$-álgebra $B$ de $V_{S}$ estabiliza $W$ si verifica las siguientes condiciones:

1. $V_{S} / B$ es plano sobre $S$.

2. $B \cdot W \subseteq W$.

Se llama estabilizador de $W$ a la máxima subálgebra de $V_{S}, B_{W}$, que estabiliza $W$.

Como en el Teorema 3.15, se tiene que si partimos de $\mathcal{Y}_{L} \in \mathcal{P} i c_{\mathcal{Y}}^{\infty}(S)$, el estabilizador $B_{W}$ coincide con el anillo de la curva $Y$ menos el divisor $\bar{y}=\pi^{-1}(x)$. Y si $\operatorname{Tr}\left(B_{W}\right) \subseteq B_{W}$ entonces $A_{W}=\operatorname{Tr}\left(B_{W}\right)$ (donde $A_{W}$ es el estabilizador de $W$ en $\mathcal{O}_{S}((z))$, Definición 3.9)

Teorema 5.20. Un punto $W \in \mathcal{G} r\left(V_{\underline{n}}^{\text {univ }}\right)(S)$ está en la imagen del morfismo de Krichever para el functor $\mathcal{P} i c_{\tilde{\mathcal{Y}}}^{\infty}$ si y sólo si:

- $B_{W} \in \mathcal{G} \mathrm{r}\left(V_{\underline{n}}^{\text {univ }}\right)(S)$ y es relativamente regular.

- $\operatorname{Tr}\left(B_{W}\right) \subset B_{W}$.

En particular el morfismo de Krichever es inyectivo.

Demostración. Para el directo basta tener en cuenta el teorema 3.15, que en este caso dice que la imagen por el morfismo de Krichever del dato $\left(Y, X, \pi, x, \bar{y}, z_{x}, z_{y_{i}}\right)$ es precisamente $B_{W}$. Para el recíproco, si $B_{W} \in \mathcal{G} r\left(V_{n}^{\text {univ }}\right)(S)$, es relativamente regular y $\operatorname{Tr}\left(B_{W}\right) \subset B_{W}$, por el teorema 5.11 recuperamos los datos $\left(Y, X, \pi, x, \bar{y}, z_{x}, z_{y_{i}}\right) \in \mathcal{C}_{v_{\underline{n}}}^{\infty}(S)$. Como $W \in$ $\mathcal{G} \mathrm{r}\left(V_{\underline{n}}^{\text {univ }}\right)(S)$ y es $B_{W}$-módulo, usando el teorema 3.19 recuperamos el haz de línea $L$ sobre $Y$ y la trivialización formal del mismo a lo largo de $\bar{y}=\pi^{-1}(x)$.

Observación 5.21. Como estamos suponiendo que la familia de curvas es lisa (y en la Grassmanniana, que el punto es relativamente regular), la pareja $\left(W, B_{W}\right)$ es maximal (Definición 3.24).

Teorema 5.22. $\mathcal{P} i c_{\tilde{\mathcal{Y}}}^{\infty}$ es representable por un subesquema de $\mathcal{G} \mathrm{r}\left(V_{\underline{n}}^{\text {univ }}\right)$.

Demostración. Se sigue de los Teoremas 5.12 y 3.22 .

Observación 5.23. Con esto completamos los resultados de la última sección de [32], pues si fijamos la estructura local de revestimiento, es decir, si nos quedamos en la fibra del morfismo $\mathcal{P} i c_{\tilde{y}}^{\infty} \rightarrow \mathbb{A}_{\underline{n}}$, obtenemos uno de los problemas de moduli allí estudiados: moduli de haces de línea sobre revestimientos finitos y punteados con trivializaciones formales, partición de ramificación fija y cierta compatibilidad entre los parámetros formales. Obtenemos entonces como caso particular la caracterización de la imagen del morfismo de Krichever para dicho moduli, lo cual nos permitirá además en capítulos posteriores calcular explicitamente el espacio tangente a este espacio de moduli en términos de operadores diferenciales y calcular un grupo que uniformice este moduli, que coincidirá con el dado en [32]. 
Observación 5.24. Para cada $W \in \operatorname{Gr}\left(k((z))^{n}\right)(S)$ podemos definir su estabilizador en $\mathcal{O}_{S}((z))^{n}, S_{W}$, como la máxima sub- $\mathcal{O}_{S}$-álgebra (no necesariamente conmutativa) de:

$$
\operatorname{End}_{\mathcal{O}_{S}((z))} \mathcal{O}_{S}((z))^{n}
$$

que estabiliza a $W$, es decir, la mayor entre las que verifican:

$$
f(W) \subseteq W \quad \text { para } \quad f \in \operatorname{End}_{\mathcal{O}_{S}((z))} \mathcal{O}_{S}((z))^{n} .
$$

El estudio recogido en [1] puede entenderse aquí como el estudio de las fibras de:

$$
\mathcal{P} i c_{\tilde{\mathcal{Y}}}^{\infty} \rightarrow \mathbb{A}
$$

hecho en términos de $S_{W}$. Desde su punto de vista, el anillo que permite recuperar la curva que reviste resulta de cortar $S_{W}$ con un álgebra de Heisenberg. Con nuestras técnicas, esta situación se recupera del siguiente modo. La imagen de la inclusión natural:

$$
V_{S} \hookrightarrow \operatorname{End}_{\mathcal{O}_{S}((z))} \mathcal{O}_{S}((z))^{n}
$$

es precisamente el álgebra de Lie del grupo de Heisenberg (véase la Definición 1.17 y la ecuación 2.0.3 y es fácil comprobar que:

$$
S_{W} \cap V_{S}=B_{W}
$$

Además, $S_{W} \cap \mathcal{O}_{S}((z))=A_{W}$, donde $A_{W}$ es el estabilizador de $W$ en $\mathcal{O}_{S}((z))$ y si partimos de un dato geométrico $\left(Y, X, \pi, x, z_{x}, z_{y_{i}}, L, \phi_{\bar{y}}\right) \in \mathcal{P} i c_{\tilde{y}}^{\infty}(S)$, por el teorema 3.15 se tiene que $A_{W}=A$, siendo $A \in \operatorname{Gr}(k((z)))(S)$ el punto que define el dato $(X, x, z)$.

Consideremos el morfismo de olvido:

$$
\begin{aligned}
\mathcal{P} i c_{\tilde{\mathcal{Y}}}^{\infty} & \rightarrow \mathcal{M}^{\infty} \\
\left(Y, X, \pi, x, \bar{y}, z_{x}, z_{\bar{y}}, L, \phi_{\bar{y}}\right) & \mapsto\left(X, x, z_{x}\right) .
\end{aligned}
$$

Se define el moduli $\mathcal{P} i c_{\tilde{\mathcal{Y}}_{X}}^{\infty}$ de haces de línea con trivialización formal sobre revestimientos de $X$ como la fibra en $\left(X, x, z_{x}\right) \in \mathcal{M}^{\infty}(k)$ del morfismo $\mathcal{P} i c_{\tilde{\mathcal{Y}}}^{\infty} \rightarrow \mathcal{M}^{\infty}$. Como en la sección anterior se tienen los siguientes resultados.

Teorema 5.25. Sea $A \in \operatorname{Gr}(k((z)))(S)$ el punto que define la terna $(X \times S, x \times S, z)$ por el morfismo de Krichever ordinario. Un punto $W \in \mathcal{G} \mathrm{r}\left(V_{n}^{\text {univ }}\right)(S)$ está en la imagen del morfismo de Krichever para $\mathcal{P} i c_{\tilde{\mathcal{Y}}_{X}}^{\infty}$ si y sólo si:

- $B_{W} \in \mathcal{G} \mathrm{r}\left(V_{\underline{n}}^{\text {univ }}\right)(S)$ y es relativamente regular.

- $A \subset B_{W}$.

En particular el morfismo de Krichever es inyectivo. 
Teorema 5.26. $\mathcal{P} i c_{\tilde{\mathcal{Y}}_{X}}^{\infty}$ es representable por un subesquema de $\mathcal{G} \mathrm{r}\left(V_{\underline{n}}^{\text {univ }}\right)$.

Observación 5.27. Fijado el polinomio $p(T)$, es decir, si nos quedamos en una fibra de $\mathcal{P} i c_{\tilde{Y}_{X}}^{\infty} \rightarrow \mathbb{A}_{\underline{n}}$, estamos en condiciones de comparar también nuestros resultados con los de [24] y afirmar que mejoramos, en algunos casos, la caracterización dada en [24]. Allí se establece una equivalencia categorial entre los datos geométricos (revestimientos con fibrados vectoriales y trivializaciones formales) y unos datos algebraicos, $\left(A_{0}, A_{n}, W\right)$, donde $A_{0}$ está relacionado con la curva de abajo, $A_{n}$ con la de arriba y $W$ con el fibrado vectorial. Desde nuestro punto de vista, esta terna queda caracterizada por $W$, eliminando así la referencia a puntos de Grassmannianas distintas.

\subsection{Sistemas integrables.}

Vamos a ver ahora que, si fijamos la curva base $X$ y nos quedamos dentro de $\mathcal{P} i c_{\mathcal{Y}_{X}}^{\infty}$, existe un sistema integrable con propiedades análogas a las del sistema de Hitchin.

Recordemos (Definición 4.8) que tenemos definido el análogo formal al morfismo de Hitchin:

$$
\operatorname{Higgs}_{X}^{\infty} \rightarrow \mathbb{A}
$$

(recordemos que nos estamos restringiendo al abierto de $\mathbb{A}$ en los que $k((z))[T] / p(T)$ es una $k((z))$-álgebra íntegra, separable y regular) y consideremos el siguiente diagrama:

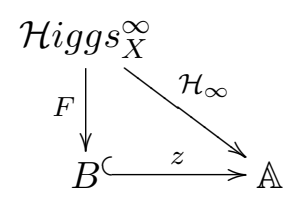

donde $B=\oplus_{i=1}^{n} \mathrm{H}^{0}\left(X, \omega_{X}^{i}\right)$ es la base del morfismo de Hitchin ordinario, $F$ es el morfismo composición:

$$
\mathcal{H i g g s}_{X}^{\infty} \rightarrow \text { Higgs }_{X} \rightarrow B
$$

que consiste en olvidar primero la trivialización formal y componer luego con el morfismo de Hitchin ordinario y $z$ es el morfismo de completación (recordemos que fijada la trivialización formal de $\mathcal{O}_{X}$ en $x$ podemos definir una trivialización formal de $\omega_{X}$ en $x$ ). Abusando de notación, denotaremos $b \in B$ a los puntos con valores en cualquier $k$-esquema.

Teniendo en cuenta los resultados clásicos ([21, 7, 12]), es fácil comprobar que $\mathcal{H}_{\infty}$ es un sistema integrable, pues se tiene que para cada punto $b \in B$ en la imagen de $\mathcal{H}_{\infty}$, la fibra de $z(b)$ por el morfismo $\mathcal{H}_{\infty}$ es en la Jacobiana $\mathcal{J}^{\infty}\left(X_{b}\right)$ de la curva espectral que define el punto $b$ (los puntos de $\mathcal{J}^{\infty}\left(X_{b}\right)$ son parejas $\left(L, \phi_{\bar{y}}\right)$ donde $L$ es un haz de línea en $X_{b}$ y $\phi_{\bar{y}}$ es una trivialización formal de $L$ a lo largo de $\bar{y}=\pi^{-1}(x)$, siendo $\pi: X_{b} \rightarrow X$ el revestimiento espectral).

De modo análogo podemos considerar el morfismo:

$$
I: \mathcal{P}_{i \mathcal{Y}_{X}}^{\infty} \rightarrow \mathcal{C o v}_{X, \underline{n}}^{\infty},
$$


que consiste en olvidar el haz de línea y su correspondiente trivialización formal, y afirmar que también es un sistema integrable, ya que la fibra sobre un punto $\left(Y, Y_{p}, z_{Y_{p}}\right)$ de $\mathcal{C}_{\text {ov }}^{\infty}, \underline{n}$ es $\mathcal{J}^{\infty}(Y)$. Si vamos un poco más lejos y tenemos en cuenta que el $k$-esquema $\mathbb{A}$ nos permite variar la estructura local de revestimiento, tenemos un diagrama:

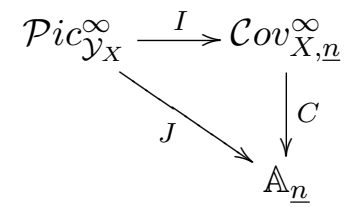

donde (por la Observación 5.10 las fibras en los puntos $p(T)$ de $\mathbb{A}_{\underline{n}}$ por el morfismo $C$ son isomorfas a los moduli $\mathcal{H}_{p(T)}^{\infty}(X)$ de revestimientos punteados sobre $X$ con trivializaciones formales estudiado en [31]. Se tiene entonces que la fibra de $J$ en $p(T) \in \mathbb{A}_{\underline{n}}$ es una familia de Jacobianas de haces de línea sobre el revestimiento universal $\mathcal{Y}_{p}$ sobre $X$ definido por $p(T)$ (y con trivializaciones formales). Al contrario de lo que pasa en el sistema de Hitchin, donde la fibra es una Jacobiana, ahora tenemos una familia de Jacobianas.

Con el propósito de establecer una analogía entre $J$ y el sistema de Hitchin (formal):

$$
\operatorname{Higgs}_{X}^{\infty} \rightarrow \mathbb{A}
$$

vamos a reducir la fibración:

$$
J: \mathcal{P}_{i c}^{\infty} \mathcal{Y}_{\mathcal{Y}_{X}}^{\infty} \rightarrow \mathbb{A}_{\underline{n}}
$$

a una fibración en la que la fibra en un punto sea una Jacobiana.

Sea $\mathcal{O}_{X, x}$ el anillo local de $X$ en el punto $x$ y consideremos el producto:

$$
\mathcal{O}_{X, x} \times \cdots \times \mathcal{O}_{X, x} .
$$

Para cada $k$-esquema $S$ definimos el functor:

$$
S \rightsquigarrow \mathcal{O}_{X \times S, x \times S} \times \cdots \times \mathcal{O}_{X \times S, x \times S} .
$$

Este functor no es representable por un $k$-esquema, pues $\mathcal{O}_{X, x}$ son las funciones en el cuerpo de funciones de $X$ sin polo en $x$ y por lo tanto:

$$
\mathcal{O}_{X, x}=\underset{D \geq 0, x \notin D}{\lim _{D}} \mathrm{H}^{0}\left(X, \mathcal{O}_{X}(D)\right) .
$$

Es decir, el functor define un Ind-esquema que denotaremos $\mathbb{O}_{x}$. Pensaremos sus puntos $\left(o_{1}, \ldots, o_{n}\right)$ como polinomios:

$$
p(T)=T^{n}+o_{1} T^{n-1}+\cdots+o_{n}
$$

y nos restringiremos en lo que sigue al abierto en que $\mathcal{O}_{X, x}[T] / p(T)$ es una $\mathcal{O}_{X, x}$-álgebra íntegra, separable y regular, que seguiremos denotando igual. 
Observación 5.28. Se tiene que $\mathbb{O}_{x} \subset \mathbb{A}$ es el morfismo de completación.

Proposición 5.29. Sea $\left(a_{1}, \ldots, a_{n}\right) \in \mathbb{A}$. Si $\left(a_{1}, \ldots, a_{n}\right) \in \mathbb{O}_{x}$, entonces existe un único revestimiento finito de grado $n, Y \rightarrow X$, y la fibra de $\mathcal{P} i c_{\mathcal{Y}_{X}}^{\infty} \rightarrow \mathbb{A}$ sobre el punto $\left(a_{1}, \ldots, a_{n}\right) \in \mathbb{O}_{x}$ es la Jacobiana $\mathcal{J}^{\infty}(Y)$.

Demostración. Si $\left(a_{1}, \ldots, a_{n}\right) \in \mathbb{O}_{x}$, entonces podemos construir una extensión finita y separable:

$$
\mathcal{O}_{X, x} \rightarrow \mathcal{O}_{X, x}[T] / p(T)
$$

de modo que $\mathcal{O}_{X, x}[T] / p(T)$ es una $\mathcal{O}_{X, x}$-álgebra íntegra. Por lo tanto, la localización en el origen de $\mathcal{O}_{X, x}[T] / p(T)$ define el cuerpo de funciones $\Sigma_{Y}$ de una única curva lisa e íntegra $Y$ y el morfismo $\Sigma_{X} \rightarrow \Sigma_{Y}$, que se obtiene a partir de $\mathcal{O}_{X, x} \rightarrow \mathcal{O}_{X, x}[T] / p(T)$, define un único revestimiento finito y separable de grado $n, Y \rightarrow X$. Es inmediato comprobar que la fibra sobre $\left(a_{1}, \ldots, a_{n}\right) \in \mathbb{O}_{x}$ de $\mathcal{P} i c_{\mathcal{Y}_{X}}^{\infty} \rightarrow \mathbb{A}$ es entonces $\mathcal{J}^{\infty}(Y)$.

Si denotamos ahora $\mathcal{P} i c_{\mathcal{Y}_{X \mid \mathbb{O}_{x}}^{\infty}}$ a la restricción de $\mathcal{P} i c_{\mathcal{Y}_{X}}^{\infty} \rightarrow \mathbb{A}$ a $\mathbb{O}_{x} \subset \mathbb{A}$ :

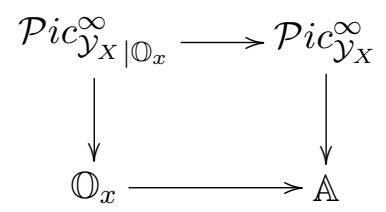

hemos visto en la proposición anterior que las fibras de $\mathcal{P} i c_{\mathcal{Y}_{X} \mid \mathbb{O}_{x}}^{\infty} \rightarrow \mathbb{O}_{x}$ son Jacobianas, luego es un sistema algebraicamente integrable y además se tiene el siguiente diagrama:

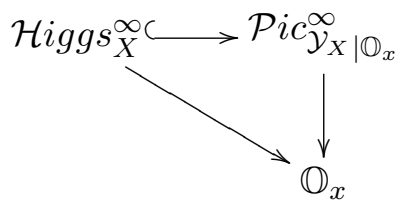

donde las fibras de sendos morfismos verticales son Jacobianas de curvas y la fibra de puntos de $\mathcal{H}_{\text {iggs }}^{\infty} \underset{X}{\infty} \rightarrow \mathbb{O}_{x}$ son las Jacobianas de los revestimientos espectrales.

Observación 5.30. Nótese que la base del morfismo de Hitchin ordinario $B=\oplus_{i=1}^{n} \mathrm{H}^{0}\left(X, \omega_{X}^{i}\right)$ está contenida en $\mathbb{O}_{x}$.

\subsection{Fibrados que son imagen directa de haces de línea.}

Veamos ahora la relación que existe entre los fibrados sobre una curva $X$ y los haces de línea en revestimientos finitos de $X$ (véase [7, 21] para los resultados clásicos). Estudiaremos también qué es lo que pasa cuando rigidificamos los espacios de moduli añadiendo trivializaciones formales. 
Denotemos $\mathcal{U}_{X}$ el stack de fibrados vectoriales de rango $n$ sobre una curva lisa, íntegra y propia sobre $k$ y por $\mathcal{P} i \mathcal{y}_{X}$ el stack de parejas $(\pi: Y \rightarrow X, L)$, donde $Y$ es una curva lisa e íntegra, $\pi$ es un morfismo finito de grado $n$ y $L$ es un haz de línea sobre $Y$.

Teorema 5.31. El morfismo natural:

$$
\begin{aligned}
\mathcal{P i c}_{\mathcal{Y}_{X}} & \rightarrow \mathcal{U}_{X} \\
(Y, L) & \mapsto \pi_{*} L
\end{aligned}
$$

es epiyectivo.

Demostración. Denotemos $\eta$ al punto genérico de $X$ y $\Sigma_{X}$ al cuerpo de funciones de $X$. Sea $E$ un punto de $\mathcal{U}_{X}$, entonces $E_{\eta}$ es un $\Sigma_{X}$-espacio vectorial de dimensión $n$. Fijemos un isomorfismo:

$$
\phi_{\eta}: E_{\eta} \stackrel{\sim}{\longrightarrow} \Sigma_{X}^{n}
$$

y sea $\tau=\left(\tau_{i j}\right)$ una matriz en $\operatorname{Gl}\left(n, \Sigma_{X}\right)$. Vía la trivialización $\phi_{\eta}, \tau$ define un isomorfismo de $\Sigma_{X}$-espacios vectoriales:

$$
\tau: E_{\eta} \simeq E_{\eta} .
$$

que extiende a un morfismo de haces de $\mathcal{O}_{X}$-módulos:

$$
\varphi: E \rightarrow E \otimes_{\mathcal{O}_{X}} \mathcal{O}_{X}(D)
$$

donde $D=\sum_{i, j} D_{\infty}\left(\tau_{i j}\right)$ es el divisor en $X$ definido por la suma de los divisores de polos de las funciones meromorfas $\tau_{i j} \in \Sigma_{X}$.

Pensando entonces la pareja $E, \varphi$ como un par de Higgs ( $\varphi$ es lo que en [7] llaman endomorfismo twisted) y aplicando la construcción espectral, es claro que obtenemos un revestimiento $\pi_{\varphi}: Y_{\varphi} \rightarrow X$ finito de grado $n$ y un haz de línea $E_{\varphi}$ sobre $Y_{\varphi}$, es decir, obtenemos en particular un punto de $\mathcal{P} i c_{\mathcal{Y}_{X}}$ tal que $\pi_{\varphi, *} E_{\varphi} \simeq E$.

Observación 5.32. Si denotamos $\overline{\mathcal{H}}$ iggs $_{X}$ al stack de pares de Higgs $(E, \varphi: E \rightarrow E \otimes L)$ sobre $X$, donde permitimos variar el haz de línea $L$ del campo de Higgs $\varphi$ (twisted endomorphism en el sentido de [7]), la demostración anterior de hecho prueba que el morfismo:

$$
\overline{\mathcal{H i g g s}}_{X} \rightarrow \mathcal{U}_{X}
$$

es epiyectivo. Claramente $\overline{\mathcal{H} i g g s}_{X} \hookrightarrow \mathcal{P} i c \mathcal{Y}_{X}$.

El mismo resultado es cierto si consideramos trivializaciones formales en el revestimiento y el haz de línea:

Corolario 5.33. La siguiente composición:

$$
\mathcal{P}_{i \mathcal{Y}_{X}}^{\infty} \rightarrow{\mathcal{P} i \mathcal{Y}_{X}}^{\infty} \rightarrow \mathcal{U}_{X}
$$

es epiyectiva. 
Demostración. Se sigue del teorema anterior teniendo en cuenta que el morfismo $\mathcal{P} i c_{\mathcal{Y}_{X}}^{\infty} \rightarrow$ $\mathcal{P} i \mathcal{Y}_{X}$, que consiste en olvidar las trivializaciones formales, es epiyectivo.

Estudiamos ahora qué sucede si añadimos además una trivialización formal al fibrado vectorial $E$ en $X$.

Sea $V=k((z))^{n}$. Recordemos que el morfismo de Krichever para el moduli $\mathcal{U}_{X}^{\infty}$ de fibrados de rango $n$ con triviliazación formal sobre el dato fijo $(X, p, z)$ es inyectivo, luego tenemos el siguiente morfismo:

$$
\operatorname{Kr} \times 1: \mathcal{U}_{X}^{\infty} \times \mathbb{A} \hookrightarrow \operatorname{Gr}(V) \times \mathbb{A} .
$$

Recordemos también que $\operatorname{Gr}(V) \times \mathbb{A} \simeq \mathcal{G r}\left(\mathcal{O}_{\mathbb{A}}((z))[T] / p(T)\right)$.

Teorema 5.34. El morfismo natural:

$$
\mathcal{P}_{i \mathcal{Y}_{X}}^{\infty} \rightarrow \mathcal{U}_{X}^{\infty} \times \mathbb{A}
$$

que a cada $\left(Y, \pi, z_{\bar{y}}, L, \phi_{\bar{y}}\right) \in \mathcal{P}_{i \mathcal{Y}_{X}}^{\infty}(S)$ asocia:

$$
\left(\left(\pi_{*} L, \pi_{*} \phi_{\bar{y}}\right), p(T)\right) \in \mathcal{U}_{X}^{\infty}(S) \times \mathbb{A}(S)
$$

es inyectivo.

Demostración. Se sigue de la inyectividad de $\mathcal{P} i c_{\mathcal{Y}_{X}}^{\infty} \hookrightarrow \operatorname{Gr}(V) \times \mathbb{A}$ (teorema 5.25 y de $\mathcal{U}_{X}^{\infty} \times \mathbb{A} \hookrightarrow \operatorname{Gr}(V) \times \mathbb{A}$, pues si dos datos $\left(Y, \pi, z_{\bar{y}}, L, \phi_{\bar{y}}\right)$ y $\left(Y^{\prime}, \pi^{\prime}, z_{\bar{y}^{\prime}}, L^{\prime}, \phi_{\bar{y}^{\prime}}\right)$ de $\mathcal{P} i c_{\mathcal{Y}_{X}}^{\infty}(S)$ definen la misma clase de equivalencia en $\mathcal{U}_{X}^{\infty}(S)$, entonces los puntos $(W, p(T))$ y $\left(W^{\prime}, p(T)^{\prime}\right)$ que definen por el morfismo

$$
\operatorname{Kr} \times 1: \mathcal{U}_{X}^{\infty} \times \mathbb{A} \hookrightarrow \operatorname{Gr}(V) \times \mathbb{A}
$$

son iguales, es decir, $W=W^{\prime}$ y $p(T)=p(T)^{\prime}$. De estas dos condiciones se deduce que los estabilizadores que definen son el mismo $B_{W}$ y vive en $\mathcal{G} \mathrm{r}\left(V_{\underline{n}}^{\text {univ }}\right)(S)$.

Así, por el teorema 5.25, tanto los revestimientos que recuperamos con $B_{W}=B_{W^{\prime}}$ como los haces de línea que recuperamos con $W=W^{\prime}$ son isomorfos y compatibles con las correspondientes trivializaciones formales, es decir, están en la misma clase de equivalencia.

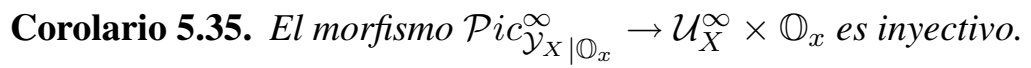

Observación 5.36. La importancia de este teorema radica en el hecho de que no es cierto si no añadimos las trivializaciones formales, hecho que se deduce de los resultados de [7]. No obstante, ilustremos con un ejemplo que $\mathcal{P} i \mathcal{Y y}_{X} \rightarrow \mathcal{U}_{X}$ no es inyectivo. En efecto, sea $N$ un haz de línea en una curva lisa, íntegra y propia $X$ sobre $k$ y consideremos los revestimientos espectrales $\pi: X_{s} \rightarrow X$ y $\pi^{\prime}: X_{s^{\prime}} \rightarrow X$ asociados a (véase la sección 4.1):

$$
s=\left(s_{1}, \ldots, s_{n}\right) \in \oplus_{i_{1}}^{n} \mathrm{H}^{0}\left(X, N^{i}\right)
$$




$$
s^{\prime}=\left(0, \ldots, 0, s^{\prime}\right) \in \oplus_{i_{1}}^{n} \mathrm{H}^{0}\left(X, N^{i}\right),
$$

con $s^{\prime} \neq 0 \mathrm{y}$, por ejemplo, algún $s_{i} \neq 0$ para $i=1, \ldots, n-1$. Se sigue que $X_{s}$ y $X_{s^{\prime}}$ son distintos revestimientos de $X$ y sin embargo, es claro que se tiene un isomorfismo de $\mathcal{O}_{X}$-módulos:

$$
\pi_{*} \mathcal{O}_{X_{s}} \simeq \mathcal{O}_{X} \oplus N \oplus N^{2} \oplus \cdots \oplus N^{n-1} \simeq \pi_{*}^{\prime} \mathcal{O}_{X_{s^{\prime}}}
$$

(como $\mathcal{O}_{X}$-álgebras no son isomorfas). Es decir, tenemos la siguiente situación:

$$
\begin{aligned}
\mathcal{P} i c_{\mathcal{Y}_{X}} & \rightarrow \mathcal{U}_{X} \\
\left(\mathcal{O}_{X_{s}}, X_{s}\right) & \mapsto\left(\pi_{*} \mathcal{O}_{X_{s}}\right) \\
\left(\mathcal{O}_{X_{s^{\prime}}}, X_{s^{\prime}}\right) & \mapsto\left(\pi_{*}^{\prime} \mathcal{O}_{X_{s^{\prime}}}\right)
\end{aligned}
$$

donde los fibrados vectoriales de rango $n$ que definen por imagen directa los haces de línea triviales $\mathcal{O}_{X_{s}}$ y $\mathcal{O}_{X_{s^{\prime}}}$ son isomorfos mientras que $\mathcal{O}_{X_{s}}$ y $\mathcal{O}_{X_{s^{\prime}}}$ (y los respectivos revestimientos que definen) son distintos. Luego $\mathcal{P} i c_{\mathcal{Y}_{X}} \rightarrow \mathcal{U}_{X}$ no es inyectivo.

Consideremos ahora el siguiente diagrama:

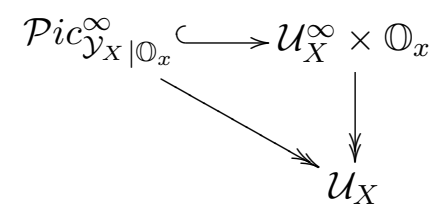

Como la flecha diagonal es epiyectiva (corolario 5.33), la razón por la que el morfismo

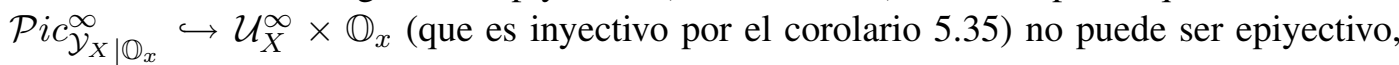
es que no toda trivialización formal de $E$ en el punto $x \in X, \widehat{E}_{x} \simeq k[[z]]^{n}$, es imagen directa (por el revestimiento finito $\pi: Y \rightarrow X$ ) de una trivialización formal del haz de línea $L$ (cuya imagen directa es $E$ ) en $\pi^{-1}(x), \widehat{L}_{\pi^{-1}(x)} \simeq k[[z]][T] / p(T)$. En términos de de los esquemas en grupos estudiados en el capítulo 1, podemos interpretar esta afirmación de la siguiente manera. Consideremos el diagrama:

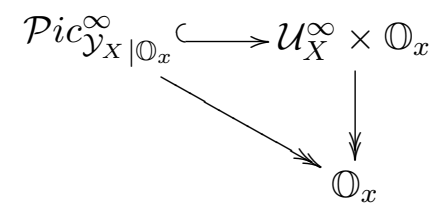

Para cada $p(T) \in \mathbb{O}$, el functor en grupos que actúa en $\mathcal{U}_{X}^{\infty}$ cambiando las trivializaciones formales del fibrado $E$ en el punto $x$ es $\mathrm{Gl}_{k[[z]]}\left(k[[z]]^{n}\right)$ (donde pensamos $\mathcal{U}_{X}^{\infty}$ como la fibra en $p(T) \operatorname{de} \mathcal{U}_{X}^{\infty} \times \mathbb{O}_{x} \rightarrow \mathbb{O}_{x}$ ).

Por otro lado, sea $\mathcal{J}^{\infty}\left(Y_{p}\right)$ la fibra de $\mathcal{P} i c_{\mathcal{Y}_{X} \mid \mathbb{O}_{x}}^{\infty} \rightarrow \mathbb{O}_{x}$ en el punto $p(T) \in \mathbb{O}$. El functor en grupos que actúa en $\mathcal{J}^{\infty}\left(Y_{p}\right)$ variando las trivializaciones formales de los haces de línea es $\Gamma_{V^{+}}\left(\right.$donde $\left.V^{+}=k[[z]][T] / p(T)\right)$. 
La imagen de $\Gamma_{V^{+}}$por la inmersión:

$$
\underline{V}^{+, *} \hookrightarrow \mathrm{Gl}_{k((z))}\left(k[[z]]^{n}\right)
$$

(donde $\underline{V}^{+, *}$ es el functor de invertibles de $V^{+}$) es el grupo de Heisenberg Heis $V^{+}$(véase la sección 1.3, que precisamente es el grupo que actúa en las trivializaciones formales del fibrado $E$ en $x$ que son imagen directa de trivializaciones formales del haz de línea del que $E$ procede. Es claro entonces que existen trivializaciones formales de $E$ en $x$ que no provienen de trivializaciones formales en $\pi^{-1}(x)$ (con $\pi: Y_{p} \rightarrow X$ ) del haz de línea $L$ tal que $E \simeq \pi_{*} L$.

En virtud de la proposición anterior y el teorema de caracterización 5.25 de $\mathcal{P} i c_{\mathcal{Y}_{X}}^{\infty}$, podemos dar un criterio para decidir cuándo un fibrado vectorial (con trivialización formal) sobre $X$ es imagen directa de un haz de línea (con trivialización formal) sobre un revestimiento finito de $X$ (con trivialización formal):

Corolario 5.37. Una pareja $(W, p(T)) \in \mathcal{U}_{X}^{\infty}(S) \times \mathbb{A}(S)$ está en la imagen de:

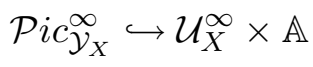

si y sólo si $B_{W_{P}} \in \mathcal{G} \mathrm{r}\left(V_{\underline{n}}^{\text {univ }}\right)(S)$ y es relativamente regular. 


\section{Parte III}

\section{Estudio infinitesimal.}





\section{Capítulo 6}

\section{Espacios tangentes y generadores locales.}

Este capítulo está dedicado al cálculo del espacio tangente de espacios de moduli, así como a demostrar que ciertos esquemas en grupos (estudiados en el primer capítulo) hacen las veces de generadores locales para dichos espacios.

En primer lugar reescribiremos algunos resultados conocidos acerca del cálculo del espacio tangente al moduli de curvas lisas o de fibrados vectoriales sobre una curva lisa. Usaremos técnicas clásicas de cohomología e hipercohomología para el cálculo de dichos espacios tangentes. Además, describiremos estos espacios tangentes dentro del espacio tangente a la Grassmanniana infinita.

Recordamos que si $K: E \rightarrow F$ un complejo de haces localmente libres sobre una curva lisa y propia sobre $k$, la hipercohomología de $K$ se define como la cohomología del complejo simple asociado al bicomplejo de Čech que define $K$ (véase [16]).

Sea $X$ una curva lisa sobre un cuerpo algebraicamente cerrado de característica nula $k$, $E$ un fibrado vectorial sobre la misma y $\mathcal{U}=\left(U_{i}\right)_{i}$ un recubrimiento abierto de $X$. A lo largo de este capítulo utilizaremos las siguientes notaciones:

$$
\begin{gathered}
U_{i}=\operatorname{Spec} A_{i} \quad U_{i j}=U_{i} \cap U_{j}=\operatorname{Spec} A_{i j} \\
M_{i}=\Gamma\left(U_{i}, E\right) \quad M_{i j}=\Gamma\left(U_{i j}, E\right)
\end{gathered}
$$

Los abiertos afines $U_{i}[\epsilon]=\operatorname{Spec} A_{i}[\epsilon]$ constituyen un recubrimiento abierto de la curva deformada $X_{\epsilon}$ (donde $A_{i}[\epsilon]=A_{i} \otimes_{k} k[\epsilon]$ y $k[\epsilon]$ denota el anillo de los números duales $\left.k[\epsilon] / \epsilon^{2}\right)$.

Sea $\mathcal{M}$ el moduli de curvas lisas, proyectivas e íntegras y $X$ un punto racional. Es conocido que el espacio tangente a $\mathcal{M}$ en $X$ vale:

$$
\mathrm{T}_{X} \mathcal{M} \simeq \mathrm{H}^{1}\left(X, \operatorname{Der}_{k}\left(\mathcal{O}_{X}, \mathcal{O}_{X}\right)\right)
$$

Sea $\mathcal{M}^{m}$ el functor de curvas punteadas con estructura de nivel al orden $m$. Un punto de $\mathcal{M}^{m}$ con valores en un $k$-esquema $S$ es una terna $\left(X, D, t_{m}\right)$ donde $X \rightarrow S$ es un morfismo 
propio y plano cuyas fibras geométricas son curvas lisas e íntegras; $\sigma: S \rightarrow X$ es una sección de $X \rightarrow S$ que considerada como divisor de Cartier $D$ sobre $X$ es liso de grado relativo 1 y plano sobre $S ; t_{m}: \mathcal{O}_{X} \rightarrow \mathcal{O}_{X} / \mathcal{O}_{X}(-m D)$ es un morfismo epiyectivo de anillos.

El moduli $\mathcal{M}^{\infty}$ (véase la Definición 3.1) puede expresarse como el límite:

$$
\mathcal{M}^{\infty} \underset{\leftarrow}{\longrightarrow} \lim _{m} \mathcal{M}^{m}
$$

y sus puntos racionales son ternas $(X, x, t)$ donde $X$ es una curva lisa, $x \in X$ un punto y $t$ es una trivialización formal:

$$
t: \widehat{\mathcal{O}}_{X, x} \stackrel{\sim}{\rightarrow} k[[z]] .
$$

Proposición 6.1. Sea $\mathcal{X}=(X, x, t)$ un punto racional de $\mathcal{M}^{\infty}$. Se tiene un isomorfismo de $k$-espacios vectoriales:

$$
\mathrm{T}_{\mathcal{X}} \mathcal{M}^{\infty} \simeq \underset{m}{\stackrel{\lim }{\leftrightarrows}} \mathrm{H}^{1}\left(X, \operatorname{Der}_{k}\left(\mathcal{O}_{X}, \mathcal{O}_{X}(-m)\right)\right)
$$

y una sucesión exacta:

$0 \rightarrow \mathrm{H}^{0}\left(X, \operatorname{Der}_{k}\left(\mathcal{O}_{X}, \mathcal{O}_{X}\right)\right) \rightarrow \underset{m}{\lim _{m}} \mathrm{H}^{0}\left(X, \operatorname{Der}_{k}\left(\mathcal{O}_{X}, \mathcal{O}_{X} / \mathcal{O}_{X}(-m)\right)\right) \rightarrow \mathrm{T}_{\mathcal{X}} \mathcal{M}^{\infty} \rightarrow \mathrm{T}_{X} \mathcal{M} \rightarrow 0$

Demostración. Es conocido que $\mathrm{T} \mathcal{M}^{m} \simeq \mathrm{H}^{1}\left(X, \operatorname{Der}_{k}\left(\mathcal{O}_{X}, \mathcal{O}_{X}(-m)\right)\right.$ ) (véase por ejemplo [46, Sec.1.2]). Para concluir basta tomar cohomología y $\varliminf_{m}$ en la sucesión exacta de haces:

$$
0 \rightarrow \operatorname{Der}_{k}\left(\mathcal{O}_{X}, \mathcal{O}_{X}(-m)\right) \rightarrow \operatorname{Der}_{k}\left(\mathcal{O}_{X}, \mathcal{O}_{X}\right) \rightarrow \operatorname{Der}_{k}\left(\mathcal{O}_{X}, \mathcal{O}_{X} / \mathcal{O}_{X}(-m)\right) \rightarrow 0
$$

y tener en cuenta que, al variar $m$, los morfismos del sistema proyectivo son epiyectivos, y por tanto, tomar $\lim _{m}$ es exacto por la derecha.

Observación 6.2. Nótese que el espacio:

$$
{\underset{m}{m}}_{\lim } \mathrm{H}^{0}\left(X, \operatorname{Der}_{k}\left(\mathcal{O}_{X}, \mathcal{O}_{X} / \mathcal{O}_{X}(-m)\right)\right) / \mathrm{H}^{0}\left(X, \operatorname{Der}_{k}\left(\mathcal{O}_{X}, \mathcal{O}_{X}\right)\right)
$$

mide las deformaciones infinitesimales de primer orden de la trivialización formal $t: \widehat{\mathcal{O}}_{X, x} \simeq k[[z]]$. Cuando $g_{X} \geq 2$, se tiene que:

$$
\mathrm{H}^{0}\left(X, \operatorname{Der}_{k}\left(\mathcal{O}_{X}, \mathcal{O}_{X}\right)\right)=0
$$

y por lo tanto, estas deformaciones vienen dadas por la completación en $x$ del haz tangente $\operatorname{Der}_{k}\left(\mathcal{O}_{X}, \mathcal{O}_{X}\right)$, es decir, por:

$$
\operatorname{Der}_{k}(k[[z]], k[[z]]) \text {. }
$$

Estas derivaciones tienen una estructura de álgebra de Lie, y de hecho son el álgebra de Lie del grupo de automorfismos de álgebras $\mathrm{G}_{k[[z]]}$ (véase la proposición 2.1. 
Observación 6.3. Puede también calcularse este espacio tangente utilizando la hipercohomología. Se tiene el siguiente resultado:

$$
\mathrm{T}_{\mathcal{X}} \mathcal{M}^{\infty} \simeq \underset{m}{\lim _{m}} \mathbb{H}^{1}\left(t_{m}^{\bullet}\right)
$$

donde $t_{m}^{\bullet}$ es el complejo epiyectivo de haces en $X$ :

$$
t_{m}^{\bullet}: \operatorname{Der}_{k}\left(\mathcal{O}_{X}, \mathcal{O}_{X}\right) \rightarrow \operatorname{Der}_{k}\left(\mathcal{O}_{X}, \mathcal{O}_{X} / \mathcal{O}_{X}(-m x)\right)
$$

definido por $t_{m}^{\bullet}(D):=t_{m} \circ D$. Es decir, deformar $\left(X, x, t_{m}\right) \in \mathcal{M}^{m}(k)$ consiste en dar parejas:

$$
\left(\left\{\theta_{i j}\right\},\left\{\beta_{i}\right\}\right) \in C^{1}\left(\mathcal{U}, \operatorname{Der}_{k}\left(\mathcal{O}_{X}\right)\right) \oplus C^{0}\left(\mathcal{U}, \operatorname{Der}_{k}\left(\mathcal{O}_{X}, \mathcal{O}_{X} / \mathcal{O}_{X}(-m x)\right)\right)
$$

tales que $\theta_{i j} \in \mathrm{H}^{1}\left(X, \operatorname{Der}_{k}\left(\mathcal{O}_{X}\right)\right)$ y $d \beta_{i}=-t_{m} \circ \theta_{i j}$, siendo $d$ la diferencial de Čech.

La relación que existe entre $\mathbb{H}^{1}\left(t_{m}\right)$ y $\mathrm{H}^{1}\left(X, \operatorname{Der}_{k}\left(\mathcal{O}_{X}, \mathcal{O}_{X}(-m)\right)\right)$ puede verse en términos de la sucesión espectral (pues el primer grupo de hipercohomología de $t_{m}$ se aglutina entre el $\mathrm{H}^{0}$ del conúcleo de $t_{m}$, que es nulo, y el $\mathrm{H}^{1}$ del núcleo) o construyendo el morfismo de connecting que resulta de tomar la sucesión exacta larga de cohomología en:

$$
0 \rightarrow \operatorname{Der}_{k}\left(\mathcal{O}_{X}, \mathcal{O}_{X}(-m)\right) \stackrel{i}{\rightarrow} \operatorname{Der}_{k}\left(\mathcal{O}_{X}\right) \stackrel{t_{m}}{\longrightarrow} \operatorname{Der}_{k}\left(\mathcal{O}_{X}, \mathcal{O}_{X} / \mathcal{O}_{X}(-m)\right) \rightarrow 0
$$

Teorema 6.4. [34 Thm. 4.11] La acción por composición de $\mathrm{G}$ en $\mathrm{Gr}(k((z)))$ induce una acción en $\mathcal{M}^{\infty}$ que es localmente transitiva.

Recordemos ahora la interpretación de este espacio tangente en términos del tangente a la Grassmanniana infinita.

Proposición 6.5. (Prop. A.21) Sea $W \in \operatorname{Gr}(V)$ un punto racional. Existe un isomorfismo de k-espacios vectoriales:

$$
\mathrm{T}_{W} \operatorname{Gr}(V) \simeq \operatorname{Hom}_{k}(W, V / W)
$$

Proposición 6.6. ([32] Prop.4.2]) Sea $(X, x, z)$ un punto racional de $\mathcal{M}^{\infty}$ y A su imagen por el morfismo de Krichever. Se tiene un isomorfismo de k-espacios vectoriales:

$$
\mathrm{T}_{(X, x, z)} \mathcal{M}^{\infty} \simeq \operatorname{Der}_{k}(A, k((z)) / A)
$$

Además:

$$
k((z)) / A \simeq \mathrm{H}^{0}\left(X-p, \omega_{X}\right)^{*} .
$$

Demostración. La caracterización de la imagen del morfismo de Krichever para el moduli $\mathcal{M}^{\infty}$ (véase [33]) nos da la manera de calcular este espacio tangente. Se tiene que:

$$
\mathrm{T}_{A} \mathcal{M}^{\infty}=\left\{\bar{A} \in \mathrm{T}_{A} \operatorname{Gr}(k((z))) \text { tales que } \bar{A} \cdot \bar{A} \subseteq \bar{A} \text { y } k[\epsilon] \subset \bar{A}\right\}
$$


donde $\epsilon^{2}=0$ y estamos escribiendo $k[\epsilon] \simeq k \oplus \epsilon \cdot k$.

Por la proposición anterior:

$$
\mathrm{T}_{A} \operatorname{Gr}(k((z))) \simeq \operatorname{Hom}_{k}(A, k((z)) / A),
$$

luego existe una aplicación $f \in \operatorname{Hom}_{k}(A, k((z)) / A)$ tal que:

$$
\bar{A}=\{a+\epsilon f(a) \mid a \in A\} .
$$

La condición $\bar{A} \cdot \bar{A} \subseteq \bar{A}$ nos dice que para todo $a, a^{\prime} \in A$, existe $a^{\prime \prime} \in A$ satisfaciendo:

$$
(a+\epsilon f(a)) \cdot\left(a^{\prime}+\epsilon f\left(a^{\prime}\right)\right)=a^{\prime \prime}+\epsilon f\left(a^{\prime \prime}\right)
$$

esto es:

$$
f\left(a^{\prime \prime}\right)=f\left(a \cdot a^{\prime}\right)=a f\left(a^{\prime}\right)+a^{\prime} f(a)
$$

Por otro lado, la condición $k[\epsilon] \subset \bar{A}$ implica que existe $a_{0} \in A$ tal que $a_{0}+\epsilon f\left(a_{0}\right)=1$, luego $f(1)=0$. En definitiva, $f$ define una derivación $D_{f} \in \operatorname{Der}_{k}(A, k((z)) / A)$ (nótese que $k((z)) / A$ es un $A$-módulo).

Para la segunda parte, consideremos la sucesión de haces:

$$
0 \rightarrow \mathcal{O}_{X}(-m x) \rightarrow \mathcal{O}_{X}(n x) \rightarrow \mathcal{O}_{X}(n x) / \mathcal{O}_{X}(-m x) \rightarrow 0
$$

tomando cohomología resulta:

$$
\begin{aligned}
0 & \rightarrow \mathrm{H}^{0}\left(X, \mathcal{O}_{X}(-m x)\right) \rightarrow \mathrm{H}^{0}\left(X, \mathcal{O}_{X}(n x)\right) \rightarrow \mathcal{O}_{X}(n x) / \mathcal{O}_{X}(-m x) \rightarrow \\
& \rightarrow \mathrm{H}^{1}\left(X, \mathcal{O}_{X}(-m x)\right) \rightarrow \mathrm{H}^{1}\left(X, \mathcal{O}_{X}(n x)\right) \rightarrow 0
\end{aligned}
$$

Se tiene que:

$$
\lim _{m} \mathrm{H}^{0}\left(X, \mathcal{O}_{X}(-m x)\right)=0 \quad \text { y } \quad \mathrm{H}^{1}\left(X, \mathcal{O}_{X}(n x)\right)=0, n>>0
$$

luego tomando ${\underset{\varliminf}{\longleftarrow}}_{m}$ y $\underline{\lim }_{\longrightarrow}$ se deduce que:

$$
0 \rightarrow A \simeq \mathrm{H}^{0}\left(X-x, \mathcal{O}_{X}\right) \rightarrow k((z)) \simeq\left(\widehat{\mathcal{O}}_{X, x}\right)_{0} \rightarrow \underset{m}{\lim _{m}} \mathrm{H}^{1}\left(X, \mathcal{O}_{X}(-m x)\right) \rightarrow 0
$$

y por lo tanto:

$$
\begin{aligned}
k((z)) / A & \simeq \underset{\stackrel{\lim }{\lim } \mathrm{H}^{1}\left(X, \mathcal{O}_{X}(-m x)\right)}{\simeq} \underset{\stackrel{\lim }{m}}{ }\left(\mathrm{H}^{0}\left(X, \omega_{X}(m x)\right)^{*}\right)= \\
& =\left(\underset{m}{\lim } \mathrm{H}^{0}\left(X, \omega_{X}(m x)\right)\right)^{*}=\mathrm{H}^{0}\left(X-x, \omega_{X}\right)^{*}
\end{aligned}
$$




\subsection{Moduli de fibrados sobre una curva lisa fija.}

Sea $\mathcal{U}_{X}$ el moduli de fibrados vectoriales de rango $n$ sobre una curva fija $X \in \mathcal{M}$ y $E$ un punto racional de $\mathcal{U}_{X}$. Es conocido que:

$$
\mathrm{T}_{E} \mathcal{U}_{X} \simeq \mathrm{H}^{1}\left(X, \mathcal{E} \operatorname{nd}_{X} E\right) .
$$

Fijemos $\left(X, x, t_{m}\right) \in \mathcal{M}^{m}$ y sea $\mathcal{U}_{X}^{m}$ el moduli de parejas $\left(E, \phi_{m}\right)$ donde $E \in \mathcal{U}_{X}$ y $\phi_{m}$ es una estructura de nivel en $E$ de orden $m$, es decir, un morfismo epiyectivo de $\mathcal{O}_{X^{-}}$ módulos:

$$
\phi_{m}: E \rightarrow\left(\mathcal{O}_{X} / \mathcal{O}_{X}(-m x)\right)^{n} \rightarrow 0
$$

siendo $n$ el rango de $E$. Denotemos $\mathcal{O}_{m x}=\mathcal{O}_{X} / \mathcal{O}_{X}(-m x)$ y nótese que, al estar concentrado en $x$, su deformación es trivial. Calculamos en este apartado el espacio tangente a $\mathcal{U}_{X}^{m}$ en $\left(E, \phi_{m}\right)$ de una manera distinta a la dada en [12].

Proposición 6.7. Sea $\left(E, \phi_{m}\right)$ un punto racional de $\mathcal{U}_{X}^{\infty}$. Se tiene un isomorfismo de $k$ espacios vectoriales:

$$
\mathrm{T}_{\left(E, \phi_{m}\right)} \mathcal{U}_{X}^{m} \simeq \mathrm{H}^{1}\left(X, \mathcal{E} \operatorname{nd}_{X} E(-m)\right) .
$$

Demostración. Deformar $\left(E, \phi_{m}\right)$ consiste en dar una familia de morfismos:

$$
\phi_{\epsilon_{i}}: M_{i}[\epsilon] \rightarrow \mathcal{O}_{m x}^{n}[\epsilon]
$$

tales que:

$$
\phi_{\epsilon_{i}}=\phi_{m}+\epsilon \delta_{i}, \quad \delta_{i} \in C^{0}\left(\mathcal{U}, \mathcal{H o m}_{X}\left(E,\left(\mathcal{O}_{X} / \mathcal{O}_{X}(-m)\right)^{n}\right)\right)
$$

y tales que sean compatibles con los morfismos $1+\epsilon \eta_{i j} \in \operatorname{End}\left(M_{i j}[\epsilon]\right)$ con lo que se construye $E_{\epsilon}$. Es decir, hemos de imponer que el siguiente diagrama sea conmutativo:

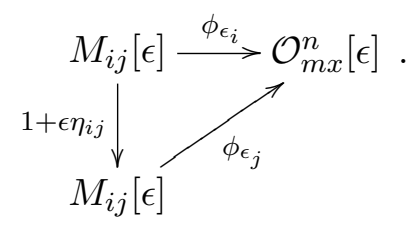

Resulta entonces:

$$
\left(\delta_{j}-\delta_{i}\right)_{\mid U_{i j}}=-\phi_{m} \circ \eta_{i j},
$$

y por lo tanto (véase la observación 6.3):

$$
\mathrm{T}_{\left(E, \phi_{m}\right)} \mathcal{U}_{X}^{m} \simeq \mathbb{H}^{1}\left(\phi_{m, *}\right) \simeq \mathrm{H}^{1}\left(X, \mathcal{E n d}_{X} E(-m)\right),
$$

siendo $\phi_{m, *}$ el siguiente complejo epiyectivo de haces de $\mathcal{O}_{X}$-módulos:

$$
\begin{aligned}
\phi_{m, *}: \mathcal{E}_{n_{X}} E & \rightarrow \mathcal{H o m}_{X}\left(E,\left(\mathcal{O}_{X} / \mathcal{O}_{X}(-m)\right)^{n}\right) \\
\eta & \mapsto \phi_{m} \circ \eta
\end{aligned}
$$


Observación 6.8. Consideremos la sucesión:

$$
0 \rightarrow \mathcal{E}_{\operatorname{nd}_{X}} E(-m) \rightarrow \mathcal{E} \operatorname{nd}_{X} E \rightarrow \mathcal{H o m}_{X}\left(E,\left(\mathcal{O}_{X} / \mathcal{O}_{X}(-m)\right)^{n}\right) \rightarrow 0
$$

Si suponemos $m>>0$ y tomamos cohomología resulta:

$$
0 \rightarrow \mathrm{T}\left\{\phi_{m}\right\} \rightarrow \mathrm{T} \mathcal{U}_{X}^{m} \rightarrow \mathrm{T} \mathcal{U}_{X} \rightarrow 0
$$

donde:

$$
\mathrm{T}\left\{\phi_{m}\right\}=\mathrm{H}^{0}\left(\mathcal{H o m}_{X}\left(E,\left(\mathcal{O}_{X} / \mathcal{O}_{X}(-m)\right)^{n}\right)\right) / \mathrm{H}^{0}\left(\mathcal{E} \operatorname{nd}_{X} E\right)
$$

mide las deformaciones infinitesimales de primer orden de las estructuras de nivel $\phi_{m}$. Si tomáramos $E$ simple, tendríamos además que $\mathrm{H}^{0}\left(\mathcal{E} \operatorname{nd}_{X} E\right)=k$.

El moduli $\mathcal{U}_{X}^{\infty}$ (cuya definición se sigue de la Definicón 3.2 fijando $(X, x, t)$ ) puede expresarse como el límite:

$$
\mathcal{U}_{X}^{\infty} \simeq \underset{m}{\lim } \mathcal{U}_{X}^{m}
$$

y sus puntos racionales corresponden a parejas $(E, \phi)$ formadas por un fibrado vectorial de rango $n$ sobre $X$, y una trivialización del mismo en $x$ :

$$
\widehat{E}_{x} \simeq \widehat{\mathcal{O}}_{X, x}^{n}
$$

Se deduce entonces que:

$$
\mathrm{T}_{(E, \phi)} \mathcal{U}_{X}^{\infty} \simeq \stackrel{\lim }{m} \mathrm{H}^{1}\left(X, \mathcal{E} \operatorname{nd}_{X} E(-m)\right)
$$

Observación 6.9. Como en la Observación 6.8, se tiene una sucesión exacta:

$$
0 \rightarrow \mathrm{T}_{\left\{\phi_{m}\right\}}\{\phi\} \rightarrow \mathrm{T}_{\left(E,\left\{\phi_{m}\right\}\right)} \mathcal{U}_{X}^{\infty} \rightarrow \mathrm{T}_{E} \mathcal{U}_{X} \rightarrow 0
$$

donde, si $E$ es simple, la deformaciones infinitesimales de primer orden, $\mathrm{T}_{\left\{\phi_{m}\right\}}\{\phi\}$, de las trivializaciones formales, son el álgebra de Lie, $\operatorname{End}_{k[[z]]}\left(k[[z]]^{n}\right)$, del grupo $\mathrm{Gl}_{k[[z]]}\left(k[[z]]^{n}\right)$ de automorfismos $k[[z]]-$ lineales de $k[[z]]^{n}$ (véase el capítulo 2).

Denotemos $V=k((z))^{n}$.

Teorema 6.10. La acción de $\mathrm{Gl}_{k((z))}(V)$ en $\mathrm{Gr}(V)$ induce una acción en $\mathcal{U}_{X}^{\infty}$ que es localmente transitiva.

Demostración. Recordemos que vía el morfismo de Krichever $\mathcal{U}_{X}^{\infty} \rightarrow \operatorname{Gr}(V)$, un punto $W \in \operatorname{Gr}(V)$ está en $\mathcal{U}_{X}^{\infty}$ si y solo si $W$ es $A$-módulo (donde $A=\mathrm{H}^{0}\left(X-x, \mathcal{O}_{X}\right)$ ).

Para ver que $\mathrm{Gl}_{k((z))}(V)$ actúa en $\mathcal{U}_{X}^{\infty}$ basta ver que $\gamma(W)$ es $A$-módulo para todo $\gamma \in$ $\mathrm{Gl}_{k((z))}(V)$. En efecto, por ser $W$ un $A$-módulo es:

$$
\gamma(a \cdot W) \subseteq \gamma(W) \quad \forall a \in A,
$$


y como $A \subset k((z))$ y $\gamma$ es un automorfismo $k((z))$-lineal de $V$ se tiene que:

$$
\gamma(a \cdot W)=a \cdot \gamma(W) \quad \forall a \in A .
$$

Resulta así que $A \gamma(W) \subseteq \gamma(W)$, es decir, $\gamma(W)$ es $A$-módulo.

Como en [34], para ver que la acción es localmente transitiva basta demostrar que el morfismo órbita:

$$
\begin{aligned}
\operatorname{Gl}_{k((z))}(V) & \rightarrow \mathcal{U}_{X}^{\infty} \\
\gamma & \mapsto \gamma(W)
\end{aligned}
$$

es epiyectivo a nivel de espacios tangentes. En efecto, consideremos la sucesión exacta de haces en $X$ :

$$
0 \rightarrow \mathcal{E} \operatorname{nd}_{X} E(-m) \rightarrow \mathcal{E} \operatorname{nd}_{X} E(\bar{m}) \rightarrow \mathcal{H} \operatorname{mom}_{X}\left(E,\left(\mathcal{O}_{X}(\bar{m}) / \mathcal{O}_{X}(-m)\right)^{n}\right) \rightarrow 0
$$

donde $m, \bar{m}>0$. Tomando cohomología, $\varliminf_{\bar{m}}$ y $\varliminf_{m}$ se tiene la siguiente sucesión exacta:

$$
0 \rightarrow \mathrm{H}^{0}\left(X-p, \mathcal{E} \operatorname{nd}_{X} E\right) \rightarrow \operatorname{End}_{k((z))} V \rightarrow \underset{m}{\lim _{m}} \mathrm{H}^{1}\left(\mathcal{E} \operatorname{nd}_{X} E(-m)\right) \rightarrow 0
$$

En virtud de las ecuaciones 6.1.1 y 2.0.1, esta sucesión se transforma en:

$$
0 \rightarrow \mathrm{H}^{0}\left(X-p, \mathcal{E} \operatorname{nd}_{X} E\right) \rightarrow \operatorname{Lie~Gl}_{k((z))}(V) \rightarrow \mathrm{T}_{\left(E,\left\{\phi_{m}\right\}_{m}\right)} \mathcal{U}_{X}^{\infty} \rightarrow 0,
$$

de donde se sigue el resultado.

Proposición 6.11. Sea $(E, \phi) \in \mathcal{U}_{X}^{\infty}$ un punto racional y $W$ su imagen por el morfismo de Krichever. Se tiene un isomorfismo de k-espacios vectoriales:

$$
\mathrm{T}_{(E, \phi)} \mathcal{U}_{X}^{\infty} \simeq \operatorname{Hom}_{A}(W, V / W),
$$

siendo A el punto racional de $\operatorname{Gr}(k((z)))$ que define la terna $(X, x, z) \in \mathcal{M}^{\infty}$ por el morfismo de Krichever. Además:

$$
V / W \simeq \mathrm{H}^{0}\left(X-p, E^{*} \otimes \omega_{X}\right)^{*} .
$$

Demostración. Sabemos que (Teorema 3.19):

$$
\mathcal{U}_{X}^{\infty}(S)=\{W \in \operatorname{Gr}(V)(S) \text { tales que } A \cdot W \subseteq W\}
$$

luego:

$$
\mathrm{T}_{W} \mathcal{U}_{X}^{\infty}=\left\{\bar{W} \in \mathrm{T}_{W} \operatorname{Gr}(V) \text { tales que } \bar{A} \cdot \bar{W} \subseteq \bar{W}\right\} .
$$

Por la Proposición 6.5, el espacio tangente a $\operatorname{Gr}(V)$ en $W$ vale:

$$
\mathrm{T}_{W} \operatorname{Gr}(V) \simeq \operatorname{Hom}_{k}(W, V / W),
$$


luego existe una aplicación $f \in \operatorname{Hom}_{k}(W, V / W)$ tal que:

$$
\bar{W}=\{w+\epsilon f(w) \mid w \in W\},
$$

(donde $\epsilon^{2}=0$ ), y dado que la terna $(X, x, z) \in \mathcal{M}^{\infty}$ está fijada, su deformación es trivial y por tanto, la derivación que induce (por la proposicón 6.6) es nula, es decir, $\bar{A}=A+\epsilon A$.

En consecuencia, la condición $\bar{A} \cdot \bar{W} \subseteq \bar{W}$ equivale a:

$$
A \cdot \bar{W} \subseteq \bar{W},
$$

lo que nos dice que para cada $a \in A$ y $w \in W$, existe $w^{\prime} \in W$ satisfaciendo:

$$
a \cdot(w+\epsilon f(w))=w^{\prime}+\epsilon f\left(w^{\prime}\right),
$$

esto es:

$$
\begin{gathered}
a w=w^{\prime} \\
f\left(w^{\prime}\right)=f(a \cdot w)=a f(w) .
\end{gathered}
$$

Es decir, hemos probado que $f$ es un morfismo de $A$-módulos. En definitiva, se tiene un ismorfismo:

$$
\mathrm{T}_{(E, \phi)} \mathcal{U}_{X}^{\infty} \simeq \operatorname{Hom}_{A}(W, V / W) .
$$

Para la segunda parte basta razonar de igual modo que en la Proposición 6.6 usando la sucesión de haces:

$$
0 \rightarrow E(-m) \rightarrow E(n) \rightarrow E(n) / E(-m) \rightarrow 0 \text {. }
$$

\subsection{Moduli de fibrados y curvas lisas.}

Denotemos $\mathcal{U}$ al functor que a cada $k$-esquema $S$ asocia la pareja $(X, E)$ formada por una familia de curvas lisas e íntegras $X \rightarrow S$ y un fibrado vectorial $E$ sobre $X$. El siguiente teorema calcula el espacio tangente a $\mathcal{U}$ en un punto racional (véase [4] o [48] para otro enfoque en el caso de haces de línea).

Teorema 6.12. Sea $(X, E) \in \mathcal{U}$ un punto racional. Se tiene un isomorfismo de k-espacios vectoriales:

$$
\mathrm{T}_{(X, E)} \mathcal{U} \simeq \mathrm{H}^{1}\left(X, \mathcal{D}_{X / k}^{1}(E, E)\right),
$$

donde $\mathcal{D}^{1}$ denota el haz de operadores diferenciales de orden $\leq 1$ con símbolo escalar (véase el apéndice C).

Demostración. Deformar la pareja $(X, E)$ equivale a deformar $E$ teniendo en cuenta que es un haz de $\mathcal{O}_{X}$-módulos, y que ahora la deformación de $\mathcal{O}_{X}$ puede no ser trivial. Por la sección 6.1 sabemos que deformar $E$ equivale a dar una familia de morfismos:

$$
1+\epsilon \eta_{i j}: M_{i j}[\epsilon] \rightarrow M_{i j}[\epsilon]
$$


donde $\eta_{i j} \in \mathrm{H}^{1}\left(X, \mathcal{E} \operatorname{nd}_{X} E\right)$; deformar $X$ consiste en dar una familia:

$$
1+\epsilon \theta_{i j}: A_{i j}[\epsilon] \rightarrow A_{i j}[\epsilon]
$$

$\operatorname{con} \theta_{i j} \in \mathrm{H}^{1}\left(\operatorname{Der}_{k}\left(\mathcal{O}_{X}\right)\right)$. Hacer estas dos familias compatibles (es decir, tener en cuenta la estructura de $\mathcal{O}_{X_{\epsilon}}$-módulo de $E_{\epsilon}$ ) equivale a la conmutatividad del siguiente diagrama:

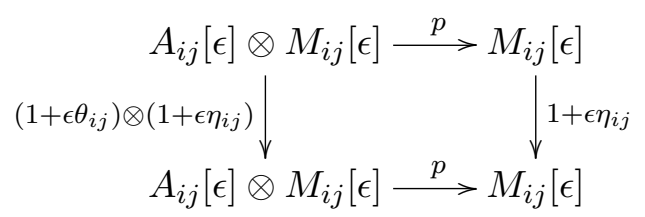

donde $p$ es la estructura de $A_{i j}[\epsilon]$-módulo de $M_{i j}[\epsilon]$. Dados $a, \bar{a} \in A_{i j}$ y $m, \bar{m} \in M_{i j}$, la composición $\left(1+\epsilon \eta_{i j}\right) \circ p$ vale:

$$
\begin{aligned}
\left(1+\epsilon \eta_{i j}\right)(p(a+\epsilon \bar{a}, m+\epsilon \bar{m})) & =\left(1+\epsilon \eta_{i j}\right)(a m+\epsilon(a \bar{m}+\bar{a} m))= \\
& =a m+\epsilon\left(a \bar{m}+\bar{a} m+\eta_{i j}(a m)\right)
\end{aligned}
$$

y la composición $p \circ\left(\left(1+\epsilon \theta_{i j}\right) \otimes\left(1+\epsilon \eta_{i j}\right)\right)$ viene dada por:

$$
\begin{aligned}
p\left(\left(1+\epsilon \theta_{i j}\right) \times\left(1+\epsilon \eta_{i j}\right)\right. & (a+\epsilon \bar{a}, m+\epsilon \bar{m}))= \\
= & p\left(a+\epsilon\left(\bar{a}+\theta_{i j}(a)\right), m+\epsilon\left(\bar{m}+\eta_{i j}(m)\right)\right)= \\
= & a m+\epsilon\left(a \bar{m}+a \eta_{i j}(m)+\bar{a} m+\theta_{i j}(a) m\right) .
\end{aligned}
$$

Como el diagrama anterior ha de ser conmutativo, tiene que verificarse que:

$$
\eta_{i j}(a m)=a \eta_{i j}(m)+\theta_{i j}(a) m .
$$

En virtud de la Proposición C.3 se concluye que:

$$
\eta_{i j} \in \mathrm{H}^{1}\left(X, \mathcal{D}_{X / k}^{1}(E, E)\right) .
$$

Además, su imagen por el morfismo símbolo $\mathcal{D}_{X / k}^{1}(E, E) \rightarrow \operatorname{Der}_{k}\left(\mathcal{O}_{X}, \mathcal{O}_{X}\right)$ es la derivación $\theta_{i j}$.

Sea $\mathcal{U}^{m}$ el functor que a cada $k$-esquema $S$ asocia los datos $\left(X, D, t_{m}, E, \phi_{m}\right)$ donde $\left(X, D, t_{m}\right) \in \mathcal{M}^{m}(S), E$ es un fibrado vectorial de rango $n$ sobre $X$ y $\phi_{m}$ es una estructura de nivel de orden $m$ de $E$.

Teorema 6.13. Sea $\mathcal{E}_{m}=\left(X, p, t_{m}, E, \phi_{m}\right) \in \mathcal{U}^{m}$ un punto racional. Se tiene un isomorfismo de k-espacios vectoriales:

$$
\mathrm{T}_{\mathcal{E}_{m}} \mathcal{U}^{m} \simeq \mathrm{H}^{1}\left(X, \mathcal{D}_{X / k}^{1}(E, E(-m))\right)
$$


Demostración. Por la Observación 6.3 tenemos que deformar $\left(X, x, t_{m}\right)$ equivale a dar parejas:

$$
\left(\left\{\theta_{i j}\right\},\left\{\beta_{i}\right\}\right) \in C^{1}\left(\mathcal{U}, \operatorname{Der}_{k}\left(\mathcal{O}_{X}\right)\right) \oplus C^{0}\left(\mathcal{U}, \operatorname{Der}_{k}\left(\mathcal{O}_{X}, \mathcal{O}_{X} / \mathcal{O}_{X}(-m x)\right)\right.
$$

tales que $\theta_{i j}$ son 1-cociclos y $\left(\beta_{j}-\beta_{i}\right)_{\mid U_{i j}}=-t_{m} \circ \theta_{i j}$. Por otra parte, la Proposición 6.7 afirma que para deformar $\left(E, \phi_{m}\right)$ hemos de dar elementos:

$$
\left(\left\{\eta_{i j}\right\},\left\{\delta_{i}\right\}\right) \in C^{1}\left(\mathcal{U}, \mathcal{E} \operatorname{nd}_{X} E\right) \oplus C^{0}\left(\mathcal{U}, \mathcal{H}_{X}\left(E,\left(\mathcal{O}_{X} / \mathcal{O}_{X}(-m x)\right)^{n}\right)\right)
$$

tales que $\eta_{i j}$ son 1-cociclos y $\left(\delta_{j}-\delta_{i}\right)_{\mid U_{i j}}=-\phi_{m} \circ \eta_{i j}$.

Deformar la quintupla al completo significa tener en cuenta la estructura de $\mathcal{O}_{X_{\epsilon}}$-módulo de $E_{\epsilon}$, que por el Teorema anterior implica que $\eta_{i j} \in \mathrm{H}^{1}\left(X, \mathcal{D}^{1}(E, E)\right)$, y que dicha estructura ha de ser compatible con las deformaciones de las estructuras de nivel $t_{m}$ y $\phi_{m}$. Es decir, el siguiente diagrama tiene que ser conmutativo:

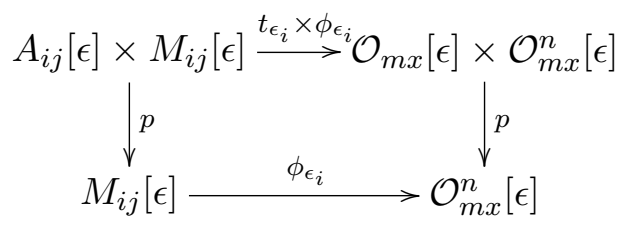

donde:

$$
\begin{array}{cc}
t_{\epsilon_{i}}=t+\epsilon \beta_{i} & \beta_{i} \in C^{0}\left(\mathcal{U}, \operatorname{Der}_{k}\left(\mathcal{O}_{X}, \mathcal{O}_{m x}\right)\right) \\
\phi_{\epsilon_{i}}=\phi_{m}+\epsilon \delta_{i} & \delta_{i} \in C^{0}\left(\mathcal{U}, \mathcal{H o m}_{X}\left(E, \mathcal{O}_{m x}^{n}\right)\right)
\end{array}
$$

y hemos denotado $\mathcal{O}_{m x}=\mathcal{O}_{X} / \mathcal{O}_{X}(-m x)$.

De esto se deduce que, para todo $a, \bar{a} \in A_{i j}, m, \bar{m} \in M_{i j}$ se tiene que:

$$
\delta_{i}(a m)=t_{m}(a) \delta_{i}(m)+\beta_{i}(\bar{a}) \phi_{m}(m),
$$

es decir,

$$
\delta_{i} \in C^{0}\left(\mathcal{U}, \mathcal{D}^{1}\left(E, \mathcal{O}_{m x}^{n}\right)\right) .
$$

Resumiendo, deformar $\left(X, p, t_{m}, E, \phi_{m}\right)$ consiste en dar parejas:

$$
\left(\left\{\eta_{i j}\right\},\left\{\delta_{i}\right\}\right) \in C^{1}\left(\mathcal{U}, \mathcal{D}^{1}(E, E)\right) \oplus C^{0}\left(\mathcal{U}, \mathcal{D}^{1}\left(E, \mathcal{O}_{m x}^{n}\right)\right)
$$

tales que $\eta_{i j}$ son 1-cociclos y $\left(\delta_{j}-\delta_{i}\right)_{\mid U_{i j}}=-\phi_{m} \circ \eta_{i j}$, que es precisamente un elemento de $\mathbb{H}^{1}\left(\phi_{m}\right)$ donde $\phi_{m}$ denota ahora el complejo epiyectivo:

$$
\begin{aligned}
\phi_{m}: \mathcal{D}^{1}(E, E) & \rightarrow \mathcal{D}^{1}\left(E,\left(\mathcal{O}_{X} / \mathcal{O}_{X}(-m)\right)^{n}\right) \\
P & \mapsto \phi_{m} \circ P
\end{aligned}
$$

Teniendo en cuenta la Observación 6.3 se sigue el resultado:

$$
\mathrm{T}_{\mathcal{E}_{m}} \mathcal{U}^{m} \simeq \mathrm{H}^{1}\left(X, \mathcal{D}^{1}(E, E(-m))\right) .
$$


Sea $\mathcal{U}^{\infty}$ el moduli de curvas y fibrados (con trivializaciones formales) definido en 3.2 . Se tiene que:

$$
\mathcal{U}^{\infty} \simeq \underset{m}{\lim } \mathcal{U}^{m}
$$

y, en un punto racional $\mathcal{E} \in \mathcal{U}_{X}^{\infty}(k)$, su espacio tangente vale:

$$
\mathrm{T}_{\mathcal{E}} \mathcal{U}^{\infty} \simeq \underset{m}{\lim _{m}} \mathrm{H}^{1}\left(X, \mathcal{D}_{X / k}^{1}(E, E(-m))\right)
$$

Como en la Observación 6.9 puede verse que se tiene una sucesión exacta:

$$
0 \rightarrow \operatorname{LieSGl}_{k[[z]]}\left(k[[z]]^{n}\right) \rightarrow \mathrm{T}_{P} \mathcal{U}^{\infty} \rightarrow \mathrm{T}_{(E, X)} \mathcal{U} \rightarrow 0,
$$

donde $\operatorname{SGl}_{k[[z]]}\left(k[[z]]^{n}\right)$ es el grupo de automorfismos semilineales (definición 1.20 y su álgebra de Lie es, en virtud de la la proposición 2.7, isomorfa a $\left.\mathcal{D}_{k[[z]] / k}^{1}\left(k[[z]]^{n}, k[z]\right]^{n}\right)$. Además, este espacio mide simultaneamente las deformaciones infinitesimales de primer orden del parámetro formal $z \mathrm{y}$ de la trivialización formal $\phi$.

Escribamos $V=k((z))^{n}$.

Teorema 6.14. El grupo $\operatorname{SGl}_{k((z))}(V)$ actúa en $\mathcal{U}^{\infty}$ y esta acción es localmente transitiva.

Demostración. Recordemos (Teorema 3.19) que los puntos de $\mathcal{U}^{\infty}$ corresponden a los puntos $W \in \operatorname{Gr}(V)$ tales $A_{W} \in \operatorname{Gr}(k((z)))$ siendo $A_{W}$ el álgebra estabilizadora de $W$ (definida en 3.9 .

Para ver que $\operatorname{SGl}_{k((z))}(V)$ actúa en $\mathcal{U}^{\infty}$ hemos de comprobar si para todo $\gamma \in \operatorname{SGl}_{k((z))}(V)$ se verifica que $\gamma(W) \in \operatorname{Gr}(V)$ y $A_{\gamma(W)} \in \operatorname{Gr}(k((z)))$. Ahora bien, como $\operatorname{SGl}_{k((z))}(V)$ actúa en $\operatorname{Gr}(V)$ se tiene que si $W \in \operatorname{Gr}(V)$ entonces $\gamma(W) \in \operatorname{Gr}(V)$. Por otro lado:

$$
\begin{aligned}
A_{\gamma(W)} & =\{a \in k((z)) \mid a \cdot \gamma(W) \subseteq \gamma(W)\}= \\
& =\left\{a \in k((z)) \mid \gamma^{-1}(a \cdot \gamma(W)) \subseteq W\right\}= \\
& =\left\{a \in k((z)) \mid g^{-1}(a) \cdot W \subseteq W\right\}=g^{-1}\left(A_{W}\right)
\end{aligned}
$$

(recordemos que por la Definición 1.20 es $\gamma(z v)=g(z) \gamma(v)$ con $g \in \mathrm{G}$ y nótese que lo mismo vale para puntos con valores en un $k$-esquema $S$ ) y como G actúa en $\operatorname{Gr}(k((z)))$, si $A_{W} \in \operatorname{Gr}(k((z)))$ entonces $g^{-1}\left(A_{W}\right) \in \operatorname{Gr}(k((z)))$. Veamos que la acción de $\operatorname{SGl}_{k((z))}(V)$ es localmente transitiva, lo que equivale a ver que el morfismo órbita:

$$
\begin{aligned}
\operatorname{SGl}_{k((z))}(V) & \rightarrow \mathcal{U}^{\infty} \\
\gamma & \mapsto \gamma(W)
\end{aligned}
$$

es epiyectivo a nivel de espacios tangentes, es decir, que el morfismo:

$$
\operatorname{Lie}\left(\operatorname{SGl}_{k((z))}(V)\right) \rightarrow \mathrm{T}_{W} \mathcal{U}^{\infty}
$$

es epiyectivo. En efecto, consideremos la siguiente sucesión exacta:

$$
0 \rightarrow \mathcal{D}_{X / k}^{1}(E, E(-m)) \rightarrow \mathcal{D}_{X / k}^{1}(E, E(\bar{m})) \stackrel{\phi_{m}}{\longrightarrow} \mathcal{D}_{X / k}^{1}\left(E,\left(\mathcal{O}_{X}(\bar{m}) / \mathcal{O}_{X}(-m)\right)^{n}\right) \rightarrow 0,
$$


tomando cohomología, ${\underset{\varliminf}{\longleftarrow} m}_{m}$ y $\underline{\lim }_{\bar{m}}$ se tiene:

$0 \rightarrow \mathrm{H}^{0}\left(X-p, \mathcal{D}_{X / k}^{1}(E)\right) \rightarrow \underset{\rightarrow}{\lim } \mathcal{D}_{X / k}^{1}\left(E,\left(\mathcal{O}_{X}(\bar{m}) / \mathcal{O}_{X}(-m)\right)^{n}\right) \rightarrow \lim _{\longleftarrow} \mathrm{H}^{1}\left(X, \mathcal{D}_{X / k}^{1}(E, E(-m))\right) \rightarrow 0$

donde:

$$
\varliminf_{\longleftarrow} \mathrm{H}^{1}\left(X, \mathcal{D}_{X / k}^{1}(E, E(-m))\right) \simeq \mathrm{TU}^{\infty}
$$

Utilizando los Teoremas 6.4 y 6.10 y el diagrama:

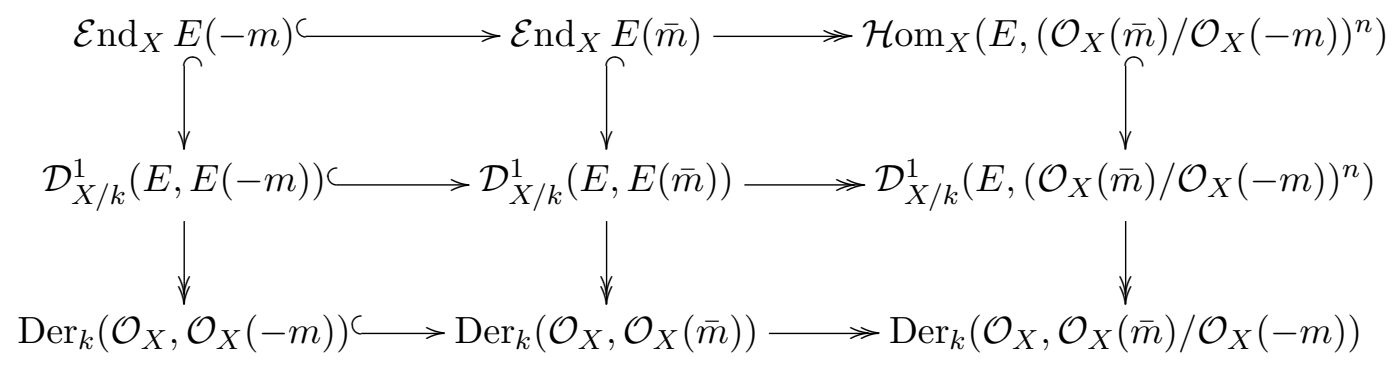

(donde filas y columnas son exactas y las epiyecciones verticales son los morfismos símbolo), obtenemos el siguiente diagrama:

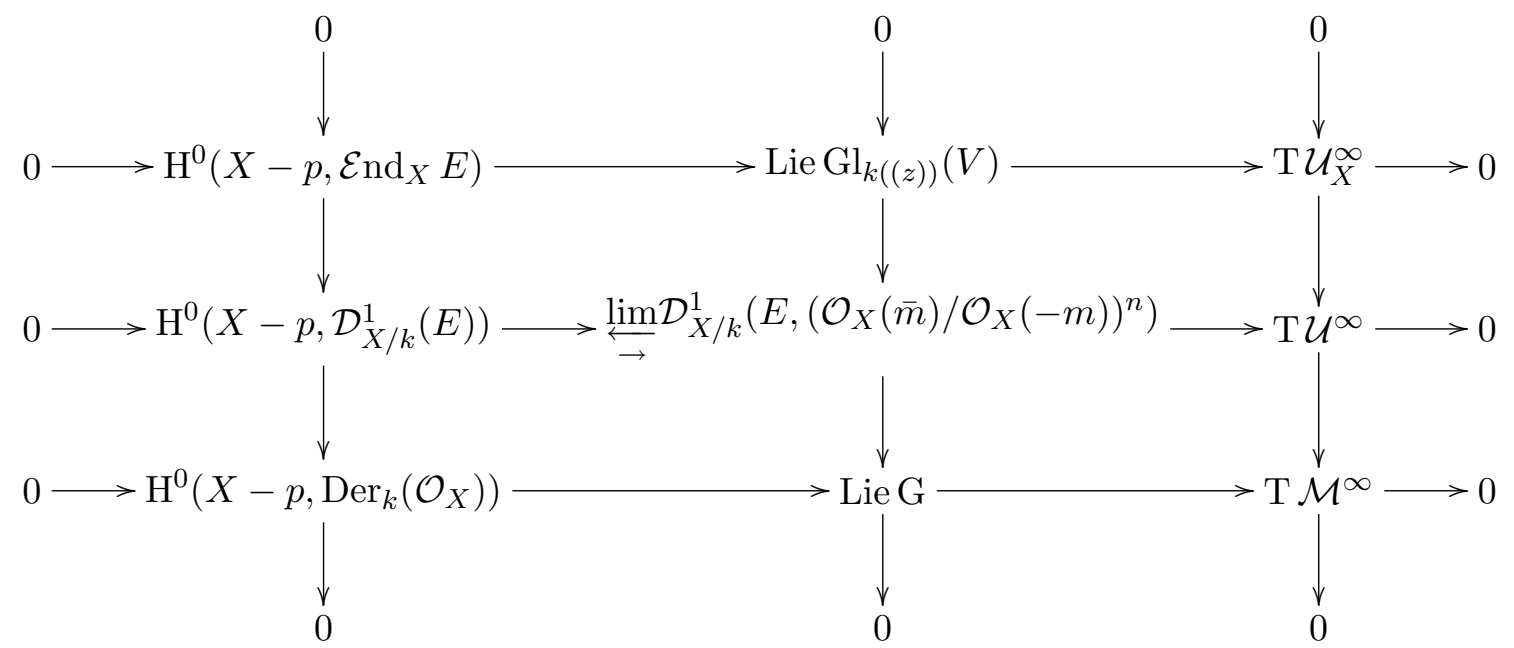

En virtud de la Observación C.6 y la Proposición 2.7, se sigue que:

$$
\underset{\leftrightarrows}{\lim _{\longrightarrow}} \mathcal{D}_{X / k}^{1}\left(E,\left(\mathcal{O}_{X}(\bar{m}) / \mathcal{O}_{X}(-m)\right)^{n}\right) \simeq \mathcal{D}_{k((z)) / k}^{1}(V, V)=\operatorname{Lie}\left(\operatorname{SGl}_{k((z))}(V)\right)
$$

De donde se deduce que el morfismo órbita 6.2.1) es epiyectivo a nivel de espacios tangentes:

$$
\operatorname{Lie}\left(\operatorname{SGl}_{k((z))}(V)\right) \rightarrow \mathrm{T}_{W} \mathcal{U}^{\infty} \rightarrow 0
$$


Observación 6.15. Con el teorema anterior hemos generalizado, al caso en el que el rango del fibrado es mayor que 1, alguno de los resultados expuestos en [4] y [32], pues damos una caracterización del espacio tangente a $\mathcal{U}^{\infty}$ en términos de operadores diferenciales (luego en particular para haces de línea, que es lo que allí se trata). Además, hemos conseguido integrar el álgebra de operadores diferenciales de Laurent, lo que nos permite dar un grupo que genera localmente el moduli y coincide, en el caso de haces de línea, con el dado en [32] (véase la Observación 1.22). El estudio de la cohomología de estos espacios de moduli, con el fin de obtener información sobre la cohomología del moduli estandar (sin trivializaciones formales), como se hace en [4], será objeto de futuras investigaciones.

Observación 6.16. La sucesión

$$
0 \rightarrow \mathrm{T}_{X}^{\infty} \rightarrow \mathrm{TU}^{\infty} \rightarrow \mathrm{T} \mathcal{M}^{\infty} \rightarrow 0
$$

puede también deducirse tomando la sucesión exacta larga de hipercohomología en la sucesión de complejos verticales (que consisten en componer con las estructuras de nivel correspondientes):

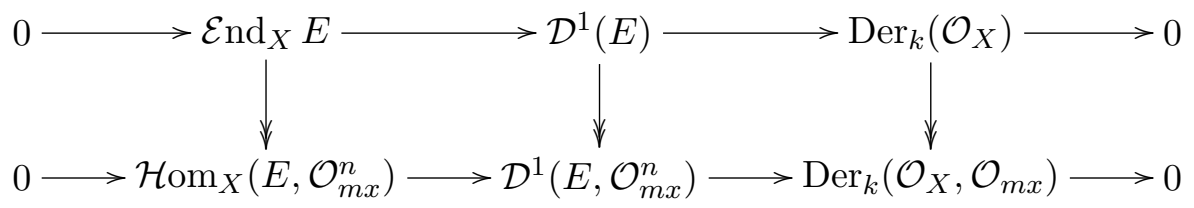

donde $\mathcal{O}_{m x}=\mathcal{O}_{X} / \mathcal{O}_{X}(-m)$.

Reinterpretamos ahora el espacio tangente en términos de la Grassmanniana infinita.

Proposición 6.17. Sea $\mathcal{E} \in \mathcal{U}^{\infty}$ un punto racional y $W \in \mathrm{Gr}(V)$ su imagen por el morfismo de Krichever. Se tiene un isomorfismo de k-espacios vectoriales:

$$
\mathrm{T}_{\mathcal{E}} \mathcal{U}^{\infty} \simeq \mathcal{D}_{A_{W} / k}^{1}(W, V / W)
$$

Demostración. Por el Teorema 3.19 y dado que la condición de regularidad es abierta:

$$
\mathrm{T}_{\mathcal{E}} \mathcal{U}^{\infty}=\left\{\bar{W} \in \mathrm{T}_{W} \operatorname{Gr}(V) \text { tales que } A_{\bar{W}} \in \mathrm{T}_{A_{W}} \operatorname{Gr}(k((z)))\right\} .
$$

Tenemos que $A_{\bar{W}}$ es $k[\epsilon]$-álgebra (donde $\epsilon^{2}=0$ y $k[\epsilon] \simeq k \oplus \epsilon \cdot k$ ) y es una deformación del estabilizador, $A_{W}$, de $W$. Se sigue entonces de la Proposición 6.6 que existe $g \in \operatorname{Der}_{k}\left(A_{W}, k((z)) / A_{W}\right)$ tal que:

$$
A_{\bar{W}}=\left\{a+\epsilon g(a), a \in A_{W}\right\} .
$$

Por otro lado, como $\bar{W} \in \mathrm{T}_{W} \operatorname{Gr}(V)$ existe $f \in \operatorname{Hom}_{k}(W, V / W)$ (Proposición 6.5) tal que:

$$
\bar{W}=\{w+\epsilon f(w), w \in W\} .
$$

Dado que $\bar{W}$ es $A_{\bar{W}}$-módulo, para cada $a \in A_{W}$ y $w \in W$, existe $w^{\prime} \in W$ tal que:

$$
(a+\epsilon g(a))(w+\epsilon f(w))=w^{\prime}+\epsilon f\left(w^{\prime}\right),
$$


igualdad que se traduce en:

$$
a f(w)+g(a) w=f(a w) .
$$

En consecuencia, $f \in \mathcal{D}_{A_{W} / k}^{1}(W, V / W)$ y $g$ es su derivación asociada vía el morfismo símbolo:

$$
\mathcal{D}_{A_{W} / k}^{1}(W, V / W) \rightarrow \operatorname{Der}_{k}\left(A_{W}, k((z)) / A_{W}\right) \rightarrow 0 .
$$

Observación 6.18. La sucesión:

$$
0 \rightarrow \operatorname{Hom}_{A}(W, V / W) \rightarrow \mathcal{D}_{A_{W} / k}^{1}(W, V / W) \rightarrow \operatorname{Der}_{k}\left(A_{W}, k((z)) / A_{W}\right) \rightarrow 0
$$

es el análogo, en términos del espacio tangente a la Grassmanniana infinita, a la sucesión:

$$
0 \rightarrow \mathrm{TU}_{X}^{\infty} \rightarrow \mathrm{T} \mathcal{U}^{\infty} \rightarrow \mathrm{T} \mathcal{M}^{\infty} \rightarrow 0
$$

obtenida en el Teorema 6.14

Así mismo, en virtud del Teorema 6.14 obtenemos un diagrama:

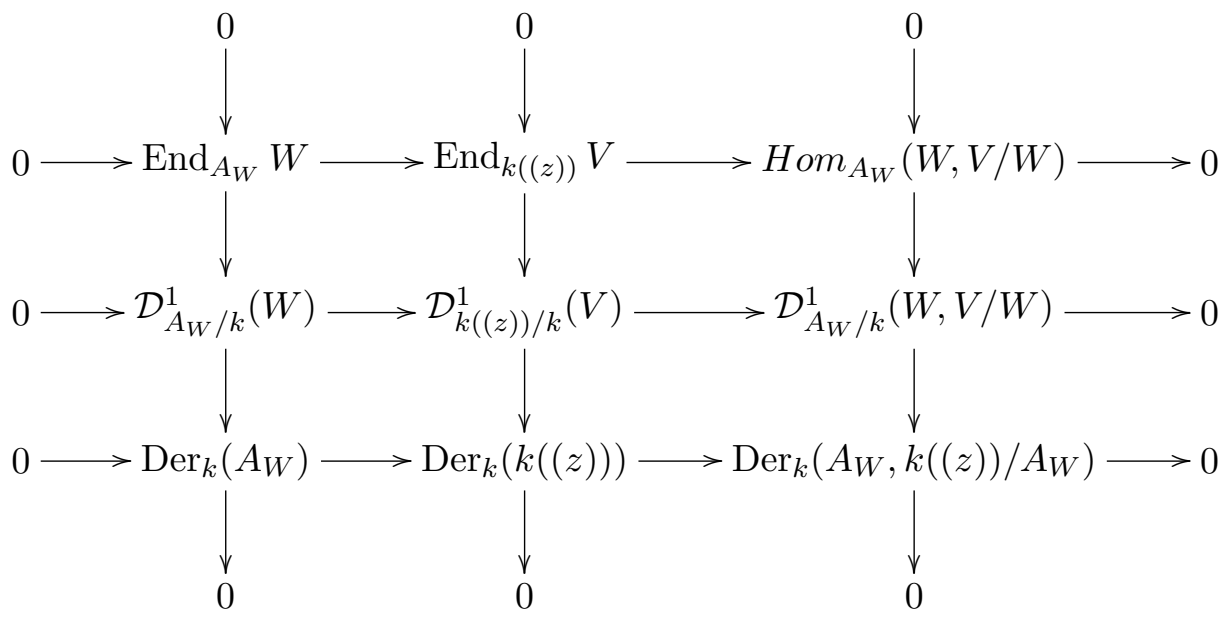

donde además:

- $\operatorname{End}_{A_{W}} W$ es el espacio tangente al estabilizador de $W$ por la acción de $\mathrm{Gl}_{k((z))}(V)$, y coincide con el álgebra estabilizadora:

$$
S_{W}:=\left\{f \in \operatorname{End}_{k((z))} V \mid f(W) \subset W\right\}
$$

de $W$ en $V$.

- $\mathcal{D}_{A_{W} / k}^{1}(W)$ es el espacio tangente al estabilizador de $W$ por la acción de $\operatorname{SGl}_{k((z))}(V)$.

- $\operatorname{Der}_{k}\left(A_{W}\right)$ es el espacio tangente al estabilizador de $A_{W}$ por la acción de $\mathrm{G}$. 


\subsection{Moduli de revestimientos.}

Denotemos $\mathcal{C}$ ov al moduli de revestimientos $\pi: Y \rightarrow X$ finitos y separables entre curvas lisas e íntegras.

Sea $\pi: Y \rightarrow X$ un punto racional de $\mathcal{C}$ ov. Siguiendo las notaciones de las secciones anteriores, sean $\mathcal{U}=\left\{U_{i}\right\}_{i}$ y $\mathcal{V}=\left\{V_{i}\right\}_{i}$ recubrimientos afines de $X$ e $Y$ respectivamente y denotemos $A_{i}=\Gamma\left(U_{i}, \mathcal{O}_{X}\right), A_{i j}=\Gamma\left(U_{i j}, \mathcal{O}_{X}\right), B_{i}=\Gamma\left(V_{i}, \mathcal{O}_{Y}\right)$ y $B_{i j}=\Gamma\left(V_{i j}, \mathcal{O}_{Y}\right)$. Sabemos que deformar $X$ equivale a dar $1+\epsilon \theta_{i j}^{X} \operatorname{con} \theta_{i j}^{X} \in \mathrm{H}^{1}\left(X, \operatorname{Der}_{k}\left(\mathcal{O}_{X}, \mathcal{O}_{X}\right)\right)$; análogamente, deformamos $Y$ dando $1+\epsilon \theta_{i j}^{Y} \operatorname{con} \theta_{i j}^{Y} \in \mathrm{H}^{1}\left(Y, \operatorname{Der}_{k}\left(\mathcal{O}_{Y}, \mathcal{O}_{Y}\right)\right)$.

Definición 6.19. Se definen las derivaciones de $Y$ equivariantes por $\pi$, $\operatorname{Der}_{k}\left(\mathcal{O}_{Y}, \mathcal{O}_{Y}\right)^{\pi}$, como las derivaciones de $\mathcal{O}_{Y}$ en $\mathcal{O}_{Y}$ compatibles con el morfismo natural $\pi: \mathcal{O}_{X} \rightarrow \pi_{*} \mathcal{O}_{Y}$, es decir, las derivaciones $\theta^{Y} \in \operatorname{Der}_{k}\left(\mathcal{O}_{Y}, \mathcal{O}_{Y}\right)$ para las que existe $\theta^{X} \in \operatorname{Der}_{k}\left(\mathcal{O}_{X}, \mathcal{O}_{X}\right)$ haciendo el siguiente diagrama conmutativo:

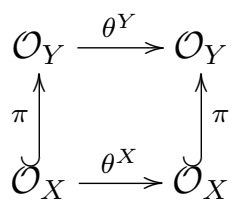

Observación 6.20. Nótese que $\operatorname{Der}_{k}\left(\mathcal{O}_{Y}, \mathcal{O}_{Y}\right)^{\pi}$ es un haz de $\mathcal{O}_{X}$-módulos, no de $\mathcal{O}_{Y^{-}}$ módulos, pues $\pi$ es sólo $\mathcal{O}_{X}$-lineal.

Proposición 6.21. Sea $\theta^{Y} \in \operatorname{Der}_{k}\left(\mathcal{O}_{Y}, \mathcal{O}_{Y}\right)^{\pi}$, entonces el siguiente diagrama es conmutativo:

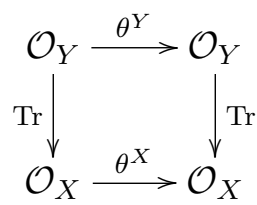

Demostración. Denotemos $\operatorname{Der}_{k}\left(\mathcal{O}_{Y}, \mathcal{O}_{Y}\right)^{\operatorname{Tr}}$ al subhaz de $\operatorname{Der}_{k}\left(\mathcal{O}_{Y}, \mathcal{O}_{Y}\right)^{\pi}$ de las derivaciones $\theta^{Y}$ tales que $\operatorname{Tr} \circ \theta^{Y}=\theta^{X} \circ \operatorname{Tr}$. Veamos que este subhaz es de hecho el total.

En primer lugar observemos que tanto $\operatorname{Der}_{k}\left(\mathcal{O}_{Y}, \mathcal{O}_{Y}\right)^{\operatorname{Tr}}$ como $\operatorname{Der}_{k}\left(\mathcal{O}_{Y}, \mathcal{O}_{Y}\right)^{\pi}$ son subhaces de $\pi_{*} \operatorname{Der}_{k}\left(\mathcal{O}_{Y}, \mathcal{O}_{Y}\right)$, y que como éste último es coherente y $\mathcal{O}_{X}$ es noetheriano, entonces también son coherentes.

En segundo lugar, como $\mathcal{O}_{X}$ es noetheriano, para todo haz de $\mathcal{O}_{X}$-módulos coherente, $M$, y todo $x \in X$ se tiene que:

$$
M \otimes \mathcal{O}_{X} \widehat{\mathcal{O}}_{X, x} \simeq \widehat{M}_{x}
$$

donde $\widehat{\mathcal{O}}_{X, x}$ denota la completación $\mathfrak{m}_{x}$-ádica, siendo $\mathfrak{m}_{x}$ el maximal del anillo local $\mathcal{O}_{X, x}$.

Para todo $x \in X$ se tiene que $\mathcal{O}_{X, x}$ es un anillo de Zarisky, lo que implica que $\widehat{\mathcal{O}}_{X, x}$ es un $\mathcal{O}_{X}$-módulo fielmente plano (véase [5, Ex.6 y Ex.7, Ch.10]). 
De esto se deduce que la inclusión natural $\operatorname{Der}_{k}\left(\mathcal{O}_{Y}, \mathcal{O}_{Y}\right)^{\operatorname{Tr}} \hookrightarrow \operatorname{Der}_{k}\left(\mathcal{O}_{Y}, \mathcal{O}_{Y}\right)^{\pi}$ es un isomorfismo de haces de $\mathcal{O}_{X}$-módulos si y sólo si lo es al completar en cada $x \in X$ :

$$
\left(\operatorname{Der}_{k}\left(\mathcal{O}_{Y}, \mathcal{O}_{Y}\right)^{\operatorname{Tr}}\right)_{x} \simeq\left(\operatorname{Der}_{k}\left(\mathcal{O}_{Y}, \mathcal{O}_{Y}\right)^{\pi}\right)_{x}
$$

Ahora bien, dado que se tienen isomorfismos canónicos:

$$
\begin{aligned}
\left(\operatorname{Der}_{k}\left(\mathcal{O}_{Y}, \mathcal{O}_{Y}\right)^{\operatorname{Tr}}\right)_{x} \simeq \operatorname{Der}_{k}\left(\widehat{\mathcal{O}}_{Y, x}, \widehat{\mathcal{O}}_{Y, x}\right)^{\operatorname{Tr}} \simeq \operatorname{Der}_{k}\left(V^{+}, V^{+}\right)^{\operatorname{Tr}} \\
\left(\operatorname{Der}_{k}\left(\mathcal{O}_{Y}, \mathcal{O}_{Y}\right)^{\pi}\right)_{x} \simeq \operatorname{Der}_{k}\left(\widehat{\mathcal{O}}_{Y, x}, \widehat{\mathcal{O}}_{Y, x}\right)^{\pi} \simeq \operatorname{Der}_{k}\left(V^{+}, V^{+}\right)^{\pi},
\end{aligned}
$$

la ecuación 6.3.1) es cierta en virtud del Corolario 2.5.

Proposición 6.22. Sea $\pi: Y \rightarrow X$ un punto racional de $\mathcal{C}$ ov, que denotaremos $(X, Y, \pi)$. Se tiene un isomorfismo de $k$-espacios vectoriales:

$$
\mathrm{T}_{(X, Y, \pi)} \mathcal{C} \text { ov } \simeq \mathrm{H}^{1}\left(X, \operatorname{Der}_{k}\left(\mathcal{O}_{Y}, \mathcal{O}_{Y}\right)^{\pi}\right) \oplus \mathrm{H}^{0}\left(X, \operatorname{Der}_{k}\left(\mathcal{O}_{X}, \mathcal{O}_{Y}\right)\right)
$$

Demostración. Deformar la terna $(X, Y, \pi)$ equivale a deformar $X$ e $Y$ de manera compatible con la deformación del morfismo $\pi$. Esta última, equivale a dar una familia:

$$
\pi+\epsilon \theta_{i}^{X Y}: A_{i}[\epsilon] \rightarrow B_{i}[\epsilon]
$$

donde, como dichos morfismos han de ser de anillos, se tiene que $\theta_{i}^{X Y} \in \mathcal{C}^{0}\left(\mathcal{U}, \operatorname{Der}_{k}\left(\mathcal{O}_{X}, \mathcal{O}_{Y}\right)\right)$.

Por lo tanto, hemos de imponer que el siguiente diagrama sea conmutativo:

$$
\begin{array}{r}
B_{i j}[\epsilon] \stackrel{1+\epsilon \theta_{i j}^{Y}}{\longrightarrow} B_{i j}[\epsilon] \\
\pi+\left.\epsilon \theta_{i}^{X Y}\right|_{U_{i j}} \uparrow \stackrel{\uparrow}{\uparrow+\left.\epsilon \theta_{i}^{X Y}\right|_{U_{i j}}} \\
A_{i j}[\epsilon] \stackrel{1+\epsilon \theta_{i j}^{X}}{\longrightarrow} A_{i j}[\epsilon]
\end{array}
$$

de donde se sigue que:

$$
\left(\theta_{j}^{X Y}-\theta_{i}^{X Y}\right)_{\mid i j}=\theta_{i j}^{Y} \circ \pi-\pi \circ \theta_{i j}^{X} .
$$

Además, como queremos que los morfismos $\pi+\epsilon \theta_{i}^{X Y}$ peguen para dar un morfismo global $\mathcal{O}_{X_{\epsilon}} \rightarrow \mathcal{O}_{Y_{\epsilon}}$, su diferencial de Čech, $\left(\theta_{j}^{X Y}-\theta_{i}^{X Y}\right)_{\mid i j}$, ha de ser nula, luego tiene que $\operatorname{ser}\left\{\theta_{i}^{X Y}\right\} \in \mathrm{H}^{0}\left(X, \operatorname{Der}_{k}\left(\mathcal{O}_{X}, \mathcal{O}_{Y}\right)\right)$.

Resumiendo, deformar la terna $(X, Y, \pi)$ equivale a dar parejas:

$$
\left(\left\{\theta_{i j}^{Y}\right\},\left\{\theta_{i}^{X Y}\right\}\right) \in \mathrm{H}^{1}\left(X, \operatorname{Der}_{k}\left(\mathcal{O}_{Y}, \mathcal{O}_{Y}\right)^{\pi}\right) \oplus \mathrm{H}^{0}\left(X, \operatorname{Der}_{k}\left(\mathcal{O}_{X}, \mathcal{O}_{Y}\right)\right) .
$$

Corolario 6.23. Si $g_{X} \geq 2$, entonces:

$$
\mathrm{T}_{(X, Y, \pi)} \mathcal{C o v} \simeq \mathrm{H}^{1}\left(X, \operatorname{Der}_{k}\left(\mathcal{O}_{Y}, \mathcal{O}_{Y}\right)^{\pi}\right) .
$$


Demostración. Obsérvese que de la demostración anterior se deduce que el espacio:

$$
\mathrm{H}^{0}\left(X, \operatorname{Der}_{k}\left(\mathcal{O}_{X}, \mathcal{O}_{Y}\right)\right)
$$

mide las deformaciones infinitesimales de primer orden del morfismo $\pi$. Como fijada la curva $Y$ el espacio de revestimientos finitos sobre $X$ con $g_{X} \geq 2$ es discreto (véase [39]), se deduce que su espacio tangente en un punto es nulo, es decir:

$$
\mathrm{H}^{0}\left(X, \operatorname{Der}_{k}\left(\mathcal{O}_{X}, \mathcal{O}_{Y}\right)\right)=0
$$

Observación 6.24. Si fijamos las dos curvas, $X$ e $Y$, la anulación de la clase:

$$
\omega(\pi)=\theta_{i j}^{Y} \circ \pi-\pi \circ \theta_{i j}^{X} \in \mathrm{H}^{1}\left(X, \operatorname{Der}_{k}\left(\mathcal{O}_{X}, \mathcal{O}_{Y}\right)\right),
$$

es una condición necesaria y suficiente para la existencia de la deformación del morfismo $\pi$ (de hecho esta clase vive en $\mathrm{H}^{1}\left(X, \operatorname{Der}_{k}\left(\mathcal{O}_{X}, \mathcal{O}_{X}\right) \otimes \operatorname{ker} \operatorname{Tr}\right.$ ), véase [47, Corollary 1.11]).

Cuando $\omega(\pi)=0$, el espacio tangente está clasificado por $\mathrm{H}^{0}\left(X, \operatorname{Der}_{k}\left(\mathcal{O}_{X}, \mathcal{O}_{Y}\right)\right)$ (véase también [20, Prop. 24.2]).

Denotemos $\mathcal{C o v}_{X}$ al moduli de revestimientos finitos y separables de curvas lisas sobre una curva fija $X$ y sea $(Y, \pi: Y \rightarrow X)$ un punto racional.

Proposición 6.25. Se tiene un isomorfismo de espacios vectoriales:

$$
\mathrm{T}_{(Y, \pi)} \mathcal{C}_{o v} \underset{ }{\simeq} \mathrm{H}^{0}\left(Y, \mathcal{O}_{R}\right)^{*}
$$

(siendo $R$ el divisor de ramificación de $\pi$ ) y una sucesión exacta:

$0 \rightarrow \mathrm{H}^{0}\left(Y, \operatorname{Der}_{k}\left(\mathcal{O}_{X}, \mathcal{O}_{Y}\right)\right) \rightarrow \mathrm{T}_{(Y, \pi)} \operatorname{Cov}_{X} \rightarrow \mathrm{H}^{1}\left(Y, \operatorname{Der}_{k}\left(\mathcal{O}_{Y}, \mathcal{O}_{Y}\right)\right) \rightarrow \mathrm{H}^{1}\left(X, \operatorname{Der}_{k}\left(\mathcal{O}_{X}, \mathcal{O}_{Y}\right)\right) \rightarrow 0$

En particular, si nos restringimos al moduli de revestimientos con comportamiento de ramificación de $\pi$ fijo, entonces el espacio tangente a $\mathcal{C}_{0} v_{X}$ en $(Y, \pi)$ es nulo.

Demostración. Consideremos la sucesión exacta de diferenciales:

$$
0 \rightarrow \pi^{*} \omega_{X} \rightarrow \omega_{Y} \rightarrow \omega_{Y / X} \rightarrow 0
$$

y tomemos duales:

$$
0 \rightarrow \operatorname{Der}_{k}\left(\mathcal{O}_{Y}, \mathcal{O}_{Y}\right) \stackrel{\circ \pi}{\longrightarrow} \operatorname{Der}_{k}\left(\mathcal{O}_{X}, \mathcal{O}_{Y}\right) \rightarrow \mathcal{E x t}^{1}\left(\omega_{Y / X}, \mathcal{O}_{Y}\right) \rightarrow 0
$$

Teniendo en cuenta que:

$$
\begin{aligned}
\mathrm{H}^{0}\left(\mathcal{E x t}^{1}\left(\omega_{Y / X}, \mathcal{O}_{Y}\right)\right) & \simeq \operatorname{Ext}^{1}\left(\omega_{Y / X}, \mathcal{O}_{Y}\right) \simeq \operatorname{Ext}^{1}\left(\mathcal{O}_{R} \otimes \omega_{Y}^{-1}, \mathcal{O}_{Y}\right) \simeq \operatorname{Ext}^{1}\left(\mathcal{O}_{R}, \omega_{Y}\right) \simeq \\
& \simeq \operatorname{Ext}^{0}\left(\omega_{Y}, \mathcal{O}_{R} \otimes \omega_{Y}\right)^{*} \simeq \operatorname{Ext}^{0}\left(\mathcal{O}_{Y}, \mathcal{O}_{R}\right)^{*} \simeq \mathrm{H}^{0}\left(Y, \mathcal{O}_{R}\right)^{*}
\end{aligned}
$$

y tomando la sucesión exacta larga en cohomología de la sucesión anterior, se deduce el enunciado (véase [19, Remark 24.6] y [18, pag.96] para más detalles). Resulta claro que si fijamos toda la ramificación de $\pi$ entonces el espacio tangente es nulo. 
Consideremos ahora el functor de olvido:

$$
\begin{aligned}
F: \mathcal{C} \text { ov } & \rightarrow \mathcal{M} \\
(X, Y, \pi) & \mapsto X .
\end{aligned}
$$

Por definición, el functor $\operatorname{Cov}_{X}$ es precisamente la fibra de $F$ en $X \in \mathcal{M}$. De esto se deduce la siguiente:

Proposición 6.26. Se tiene una sucesión exacta de espacios vectoriales:

$$
0 \rightarrow \mathrm{T}_{(Y, \pi)} \operatorname{Cov}_{X} \rightarrow \mathrm{T}_{(X, Y, \pi)} \mathcal{C o v} \rightarrow \mathrm{T}_{X} \mathcal{M} \rightarrow 0
$$

En particular, si nos restringimos al moduli de revestimientos con comportamiento de ramificación de $\pi$ fijo, se tiene que:

$$
\begin{gathered}
\mathrm{T}_{(Y, \pi)} \mathcal{C}_{\operatorname{Dov}_{X}}=\{0\} \\
\mathrm{T}_{(X, Y, \pi)} \mathcal{C} \text { ov } \simeq \mathrm{T}_{X} \mathcal{M}
\end{gathered}
$$

Sea ahora $\mathcal{C}_{0} v_{n}^{m}$ el moduli de revestimientos punteados y con estructura de nivel de orden $m$ e índices de ramificación $\underline{n}=\left(n_{1}, \ldots, n_{r}\right)$. Un punto racional consiste en dar un un conjunto de datos $\mathcal{Y}^{m}=\left(X, Y, \pi, x, \bar{y}, z_{x}^{m}, z_{\bar{y}}^{m}\right)$, donde $\pi: Y \rightarrow X$ es un punto racional de $\operatorname{Cov}, x \in X, z_{x}^{m}$ es una estructura de nivel de orden $m$ de $X$ en $x$, i.e., un morfismo epiyectivo de anillos:

$$
z_{x}^{m}: \mathcal{O}_{X} \rightarrow \mathcal{O}_{X} / \mathcal{O}_{X}(-m x)
$$

$z_{\bar{y}}^{m}$ es una estructura de nivel de $Y$ en $\bar{y}=\pi^{-1}(x)=n_{1} y_{1}+\cdots+n_{r} y_{r}$ (nótese que estamos fijando la ramificación en $x \in X$, no de todo $\pi$ ).

Como en las secciones anteriores puede verse que si $g_{X} \geq 2$ entonces:

$$
\mathrm{T}_{\mathcal{Y}^{m}} \mathcal{C}_{o v} v_{\underline{n}}^{m} \simeq \mathrm{H}^{1}\left(X, \operatorname{Der}_{k}\left(\mathcal{O}_{Y}, \mathcal{O}_{Y}(-m \bar{y})\right)^{\pi}\right)
$$

y por lo tanto:

$$
\mathrm{T}_{\mathcal{Y}} \mathcal{C}_{0} v_{\underline{n}}^{\infty} \simeq \underbrace{\lim }_{m} \mathrm{H}^{1}\left(X, \operatorname{Der}_{k}\left(\mathcal{O}_{Y}, \mathcal{O}_{Y}(-m \bar{y})\right)^{\pi}\right)
$$

donde $\mathcal{C}_{0} v_{\underline{n}}^{\infty}$ es el moduli de revestimientos con trivializaciones formales e $\mathcal{Y}$ es un punto racional del mismo (véase la Definición 5.6.

Proposición 6.27. Se tiene una sucesión exacta de espacios vectoriales:

$0 \rightarrow \mathrm{H}^{0}\left(X, \operatorname{Der}_{k}\left(\mathcal{O}_{Y}, \mathcal{O}_{Y}\right)^{\pi}\right) \rightarrow \underset{m}{\lim } \operatorname{Der}_{k}\left(\mathcal{O}_{Y}, \mathcal{O}_{Y} / \mathcal{O}_{Y}(-m)\right)^{\pi} \rightarrow \mathrm{T}_{\mathcal{Y}} \operatorname{Cov}_{\underline{n}}^{\infty} \rightarrow \mathrm{T}_{(X, Y, \pi)} \operatorname{Cov}_{\underline{n}} \rightarrow 0$.

Demostración. Basta tener en cuenta los resultados anteriores y tomar cohomología y $\varliminf_{m}$ en la sucesión:

$$
0 \rightarrow \operatorname{Der}_{k}\left(\mathcal{O}_{Y}, \mathcal{O}_{Y}(-m)\right)^{\pi} \rightarrow \operatorname{Der}_{k}\left(\mathcal{O}_{Y}, \mathcal{O}_{Y}\right)^{\pi} \rightarrow \operatorname{Der}_{k}\left(\mathcal{O}_{Y}, \mathcal{O}_{Y} / \mathcal{O}_{Y}(-m)\right)^{\pi} \rightarrow 0
$$

Nótese además que:

$$
\left({\underset{m}{m}}_{\lim } \operatorname{Der}_{k}\left(\mathcal{O}_{Y}, \mathcal{O}_{Y} / \mathcal{O}_{Y}(-m)\right)^{\pi}\right) / \mathrm{H}^{0}\left(X, \operatorname{Der}_{k}\left(\mathcal{O}_{Y}, \mathcal{O}_{Y}\right)^{\pi}\right)
$$

mide las deformaciones infinitesimales de primer orden de las trivializaciones formales $z_{x} \mathrm{y}$ $z_{y}$. 
Observación 6.28. Si $g_{Y} \geq 2$, entonces:

$$
\mathrm{H}^{0}\left(X, \operatorname{Der}_{k}\left(\mathcal{O}_{Y}, \mathcal{O}_{Y}\right)^{\pi}\right)=0
$$

y por lo tanto, las deformaciones infinitesimales de primer orden de las trivializaciones formales son:

$$
\varliminf_{m} \operatorname{Der}_{k}\left(\mathcal{O}_{Y}, \mathcal{O}_{Y} / \mathcal{O}_{Y}(-m)\right)^{\pi} \simeq\left(\prod_{i=1}^{r} k\left[\left[z_{i}\right]\right] \partial_{z_{i}}\right)^{\pi} \simeq \operatorname{Lie~}_{k[[z]]}^{V^{+}}
$$

donde denotamos $V^{+}$al producto $\prod_{i=1}^{r} k\left[\left[z_{i}\right]\right]$ y $\mathrm{G}_{k[[z]]}^{V^{+}}$al grupo de automorfismos de $V^{+}$ compatibles con la estructura de $k[[z]]$-álgebra (véase la Definición 1.8 y el Corolario 2.5.

Recordemos que denotamos $V_{\underline{n}}$ a $k\left(\left(z_{1}\right)\right) \times \ldots k\left(\left(z_{r}\right)\right)$ con la siguiente estructura de $k((z))$-álgebra (véase la ecuación $\sqrt{1.2 .2})$ ):

$$
\begin{aligned}
k((z)) & \rightarrow k\left(\left(z_{1}\right)\right) \times \cdots \times k\left(\left(z_{r}\right)\right) \\
z & \mapsto\left(z_{1}^{n_{1}}, \ldots, z_{r}^{n_{r}}\right)
\end{aligned}
$$

y pensamos $V_{\underline{n}, p}$ como $k((z))[T] / p_{1}(T) \times \cdots \times k((z))[T] / p_{r}(T)$ con la estructura (véase la ecuación (1.2.4)):

$$
\begin{aligned}
k((z)) & \rightarrow k((z))[T] / p_{1}(T) \times \cdots \times k((z))[T] / p_{r}(T) \\
z & \mapsto\left(T^{n_{1}} u_{1}(z, T)^{-1}, \ldots, T^{n_{r}} u_{r}(z, T)^{-1}\right)
\end{aligned}
$$

Los grupos de automorfismos compatibles con la estructura de $k((z))$-álgebra (Definición 1.8 se escriben $\mathrm{G}_{\underline{\underline{n}}}$ y $\mathrm{G}_{\underline{\underline{n}}, p}$ respectivamente. Recordemos también que por la Proposición 1.10 estos grupos son isomorfos.

Veamos ahora que la importancia de este grupo de automorfismos radica en el hecho de que ejerce las veces de generador local para el espacio de moduli $\mathcal{C}_{o v} v_{\underline{n}}^{\infty}$.

Teorema 6.29. El grupo $\mathrm{G}_{\underline{n}}$ actúa en $\mathcal{C}_{\underline{n}} v_{\underline{n}}^{\infty}$ y esta acción es localmente transitiva.

Demostración. Por el Teorema 5.11, un punto racional $B \in \mathcal{G} \mathrm{r}\left(V_{\underline{n}}^{\text {univ }}\right)$ yace en $\mathcal{C}_{\text {ov }}^{\infty}(k)$, si y sólo si $B$ es una sub- $k$-álgebra regular de $V_{\underline{n}, p}$ y $\operatorname{Tr}(B) \subseteq B$. Como hemos dicho, el punto racional $B$ ha de vivir en $V_{\underline{n}, p}$, luego a priori, el grupo que debiera actuar es $\mathrm{G}_{\underline{n}, p}$. Ahora bien, en virtud del isomorfismo natural $\mathrm{G}_{\underline{n}} \simeq \mathrm{G}_{\underline{n}, p}$ de la Proposición 1.10, basta ver que $\mathrm{G}_{\underline{n}}$ actúa en $\mathcal{C}_{0} v_{\underline{n}}^{\infty}$. En definitiva, hemos de comprobar si se verifica $\bar{g}(B) \in \mathcal{G} \mathrm{r}\left(V_{\underline{n}}^{\text {univ }}\right)$, $\bar{g}(B) \cdot \bar{g}(B) \subseteq \bar{g}(B)$ y $\operatorname{Tr}(\bar{g}(B)) \subseteq \bar{g}(B)$ para todo $\bar{g} \in \mathrm{G}_{\underline{n}}$. En efecto:

- Es fácil ver que $\mathrm{G}_{\underline{n}}$ actúa en $\mathcal{G} \mathrm{r}\left(V_{\underline{n}}^{\text {univ }}\right)$, luego $\bar{g}(B) \in \mathcal{G} \mathrm{r}\left(V_{\underline{n}}^{\text {univ }}\right)$.

- Como $\bar{g}$ es un automorfismo de $k$-álgebras:

$$
\bar{g}(B) \cdot \bar{g}(B)=\bar{g}(B \cdot B) \subseteq \bar{g}(B) .
$$


- Por la Proposición 1.12 se tiene que:

$$
\bar{g} \in \mathrm{G}_{\underline{\underline{n}}} \Longleftrightarrow \operatorname{Tr} \circ \bar{g}=\bar{g} \circ \operatorname{Tr},
$$

luego:

$$
\operatorname{Tr}(\bar{g}(B))=\bar{g}(\operatorname{Tr}(B)) \subseteq \bar{g}(B)
$$

Para ver que la acción es localmente transitiva basta ver que el morfismo órbita:

$$
\begin{aligned}
\mathrm{G}_{\underline{n}} & \rightarrow \mathcal{C}_{o v} v_{\underline{n}}^{\infty} \\
\bar{g} & \mapsto \bar{g}(B)
\end{aligned}
$$

es epiyectivo a nivel de espacios tangentes, esto es, si se verifica:

$$
\text { Lie } \mathrm{G}_{\underline{n}} \rightarrow \mathrm{T}_{B} \mathcal{C}_{\text {ov }}^{\infty} \rightarrow 0 .
$$

Pero esto se sigue tomando cohomología, $\underline{\lim }_{\bar{m}} \mathrm{y} \varliminf_{\leftarrow}$ en la sucesión:

$$
0 \rightarrow \operatorname{Der}_{k}\left(\mathcal{O}_{Y}, \mathcal{O}_{Y}(-m)\right)^{\pi} \rightarrow \operatorname{Der}_{k}\left(\mathcal{O}_{Y}, \mathcal{O}_{Y}(\bar{m})\right)^{\pi} \rightarrow \operatorname{Der}_{k}\left(\mathcal{O}_{Y}, \mathcal{O}_{Y}(\bar{m}) / \mathcal{O}_{Y}(-m)\right)^{\pi} \rightarrow 0
$$

y teniendo en cuenta que:

$$
\underset{\vec{m}}{\lim _{m}} \lim _{m} \operatorname{Der}_{k}\left(\mathcal{O}_{Y}, \mathcal{O}_{Y}(\bar{m}) / \mathcal{O}_{Y}(-m)\right)^{\pi} \simeq \operatorname{Der}_{k}\left(V_{\underline{n}}, V_{\underline{n}}\right)^{\pi} \simeq \operatorname{Lie}_{\underline{n}}
$$

(véase el Corolario 2.5).

Observación 6.30. Para hacer válida la proposición en el caso de puntos con valores en un esquema $S$ basta usar la topología étale (véase la Observación 1.11).

Otro modo de solventar este problema técnico es el siguiente. Definamos, como en 1.8, el functor en grupos $\mathrm{G}_{\underline{n}}^{\text {univ }}$ de automorfismos de álgebras de $V_{\underline{n}}^{\text {univ }}$ compatibles con la estructura de $\mathcal{O}_{\mathbb{A}_{n}}((z))$-álgebra definida en 5.1.4). De manera análoga a la Proposición anterior, puede verse que $\mathrm{G}_{\underline{n}}^{\text {univ }}$ actúa en $\mathcal{C}_{0} v_{\underline{n}}^{\infty}$ y dicha acción es localmente transitiva. En particular, para puntos racionales se recupera el resultado de la Proposición y obtenemos directamente la generalización a puntos con valores en un $\mathbb{A}_{\underline{\underline{n}}}$-esquema $S$. Se verifica además que, para puntos racionales o trabajando en la topología étale, el morfismo natural:

$$
\mathrm{G}_{\underline{n}}^{u n i v} \rightarrow \mathbb{A}_{\underline{n}}
$$

es un fibrado principal trivial de grupo $\mathrm{G}_{\underline{n}}$.

El objetivo de escribir la proposición anterior en términos sólo de $G_{\underline{n}}$, no es otro que destacar que para puntos racionales (o si trabajamos en la topología étale), no son necesarias estas complicaciones técnicas, ni hacer uso de un grupo "tan grande" como $\mathrm{G}_{\underline{n}}^{\text {univ }}$.

Conviene también recordar aquí que el morfismo traza, Tr, cambia de base. 
Interpretamos ahora el espacio tangente a $\mathcal{C}_{0} v_{n}^{\infty}$ en términos de la Grassmanniana infinita relativa $\mathcal{G} \mathrm{r}\left(V_{\underline{n}}^{\text {univ }}\right)$ en la que se sumerge. Para ello, necesitaremos primero calcular el espacio tangente a $\mathcal{G} \mathrm{r}\left(V_{\underline{n}}^{\text {univ }}\right)$. Recordemos que se tiene un isomorfismo (véase la ecuación (4.3.1)):

$$
\mathcal{G} r\left(V_{\underline{n}}^{\text {univ }}\right) \simeq \operatorname{Gr}(V) \times \mathbb{A}_{\underline{n}}
$$

de este modo, pensaremos cada punto racional $B$ de $\mathcal{G} \mathrm{r}\left(V_{\underline{n}}^{\text {univ }}\right)$ como una pareja de puntos racionales $\left(B_{p}, p(T)\right)$ en $\operatorname{Gr}\left(V_{\underline{n}, p}\right) \times \mathbb{A}_{\underline{n}}$.

Proposición 6.31. Sea $B$ un punto racional de $\mathcal{G} r\left(V_{n}^{\text {univ }}\right)$ y $p(T) \in \mathbb{A}_{\underline{n}}$ el punto que define por el morfismo $\mathcal{G} \mathrm{r}\left(V_{\underline{n}}^{\text {univ }}\right) \rightarrow \mathbb{A}_{\underline{n}}$. Se tiene un isomorfismo de espacios vectoriales:

$$
\mathrm{T}_{B} \mathcal{G} \mathrm{r}\left(V_{\underline{\underline{n}}}^{\text {univ }}\right) \simeq \operatorname{Hom}_{k}\left(B_{p}, V_{\underline{n}, p} / B_{p}\right) \oplus \mathrm{T}_{p(T)} \mathbb{A}_{\underline{n}}
$$

Demostración. Basta tener en cuenta que $\mathcal{G} r\left(V_{\underline{n}}^{\text {univ }}\right) \rightarrow \mathbb{A}_{\underline{n}}$ es una fibración trivial de Grassmannianas y que el tangente a la fibra es, por la Proposición 6.5. isomorfo a $\operatorname{Hom}_{k}\left(B_{p}, V_{\underline{n}, p} / B_{p}\right)$.

Observación 6.32. Nótese que como $\mathbb{A}_{\underline{n}}$ es un espacio afín, se tiene que $\mathrm{T}_{p(T)} \mathbb{A}_{\underline{n}} \simeq \mathbb{A}_{\underline{n}}$.

Definición 6.33. Sea $B$ una subálgebra de una $k((z))$-álgebra $V$ tal que $\operatorname{Tr}(B) \subseteq B$. Defini$\operatorname{mos} \operatorname{Der}_{k}(B, V / B)^{\text {Tr }}$ como el conjunto de derivaciones equivariantes por la traza, es decir, derivaciones $D: B \rightarrow V / B$ haciendo conmutativo el siguiente diagrama:

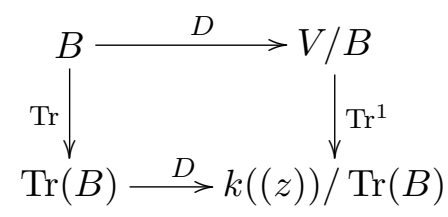

donde $\operatorname{Tr}^{1}$ es la aplicación inducida por la traza (que está bien definida porque $\operatorname{Tr}(B) \subseteq B$ ).

Teorema 6.34. Sea $\mathcal{Y}$ un punto racional de $\mathcal{C}_{0} v_{\underline{n}}^{\infty}$, B su imagen por el morfismo de Krichever y sea $p(T) \in \mathbb{A}_{\underline{n}}$ el punto que define por el morfismo $C: \mathcal{C}_{\underline{n}}^{\infty} \rightarrow \mathbb{A}_{\underline{n}}$. Se tiene un isomorfismo de k-espacios vectoriales:

$$
\mathrm{T}_{\mathcal{Y}} \mathcal{C o v}_{\underline{n}}^{\infty} \simeq \operatorname{Der}_{k}\left(B_{p}, V_{\underline{n}, p} / B_{p}\right)^{\operatorname{Tr}} \oplus \mathrm{T}_{p(T)} \operatorname{Im}(C) .
$$

Demostración. En primer lugar, recordemos que estamos identificando cada punto $B$ de $\mathcal{G} \mathrm{r}\left(V_{\underline{n}}^{\text {univ }}\right)$ con la pareja $\left(B_{p}, p(T)\right)$ de $\operatorname{Gr}\left(V_{\underline{n}, p}\right) \times \mathbb{A}_{\underline{n}}$. Observemos que el morfismo:

$$
C: \operatorname{Cov}_{\underline{n}}^{\infty} \rightarrow \mathbb{A}_{\underline{n}}
$$

induce un morfismo a nivel de espacios tangentes:

$$
d_{p(T)} C: \mathrm{T}_{\mathcal{Y}} \mathcal{C}_{0} v_{\underline{n}}^{\infty} \rightarrow \mathrm{T}_{p(T)} \mathbb{A}_{\underline{n}}
$$


cuyo núcleo denotaremos $\mathrm{T}_{\mathcal{Y}_{p}} \operatorname{Cov}_{\underline{n}, p}^{\infty}$. Con esto, se obtiene un isomorfismo:

$$
\mathrm{T}_{\mathcal{Y}} \mathcal{C}_{0} v_{\underline{n}}^{\infty} \simeq \mathrm{T}_{\mathcal{Y}_{p}} \mathcal{C o v}_{\underline{n}, p}^{\infty} \oplus \mathrm{T}_{p(T)} \operatorname{Im}(C) .
$$

Para deducir el resultado basta ver que:

$$
\mathrm{T}_{\mathcal{Y}_{p}} \operatorname{Cov}_{\underline{n}, p}^{\infty} \simeq \operatorname{Der}_{k}\left(B_{p}, V_{\underline{n}, p} / B_{p}\right)^{\mathrm{Tr}}
$$

En efecto, el espacio $\mathcal{C}_{0 v}^{\infty}{ }_{n, p(T)}^{\infty}$ (fibra de $C$ en $p(T)$ ) es el moduli de revestimientos con trivializaciones formales y compatibilidad fija entre los parámetros formales (en su expresión más sencilla, cuando $p(T)=\left(T^{n_{1}}-z, \ldots, T^{n_{r}}-z\right)$, dicha fibra es el moduli tratado en [32, 31] y por tanto, el siguiente cálculo puede deducirse de [32, Thm.3.4.9]). Usando el Teorema 5.11 se tiene que, vía el morfismo de Krichever, los puntos de $\mathrm{T}_{\mathcal{Y}_{p}} \mathcal{C}_{0} v_{\underline{n}, p}^{\infty}$ se identifican con los puntos $\bar{B}_{p}$ de $\mathrm{T}_{B_{p}} \operatorname{Gr}\left(V_{n, p}\right)$ tales que $k[\epsilon] \subset \bar{B}_{p}$ (con $\epsilon^{2}=0$ ), $\bar{B}_{p} \cdot \bar{B}_{p} \subseteq$ $\bar{B}_{p}$ y $\operatorname{Tr}\left(\bar{B}_{p}\right) \subset \bar{B}_{p}$. Como en el Teorema 6.6, puede verse que las dos primeras condiciones implican que existe $g \in \operatorname{Der}_{k}\left(B_{p}, V_{\underline{n}, p} / B_{p}\right)$ tal que:

$$
\bar{B}_{p}=\left\{b+g(b) \text { con } b \in B_{p}\right\} .
$$

Por último, la condición $\operatorname{Tr}\left(\bar{B}_{p}\right) \subseteq \bar{B}_{p}$ equivale a que para cada $b \in B_{p}$ exista $b^{\prime} \in B_{p}$ satisfaciendo:

$$
\operatorname{Tr}(b+\epsilon f(b))=b^{\prime}+\epsilon f\left(b^{\prime}\right),
$$

y como $\operatorname{Tr}\left(B_{p}\right) \subseteq B_{p}$ por ser $B_{p}$ un punto de $\mathcal{C}_{0} v_{\underline{n}, p}^{\infty}$, esta condición se traduce en:

$$
\operatorname{Tr}^{1}(f(b))=f(\operatorname{Tr}(b)) \quad \forall b \in B_{p} .
$$

En definitiva, $g \in \operatorname{Der}_{k}\left(B_{p}, V_{\underline{n}, p} / B_{p}\right)^{\operatorname{Tr}}$.

Observación 6.35. El morfismo $\operatorname{Tr}^{1}$ se corresponde con:

$$
\operatorname{Tr}^{1}: \mathrm{H}^{0}\left(Y-\bar{y}, \omega_{Y}\right)^{*} \rightarrow \mathrm{H}^{0}\left(X-x, \omega_{X}\right)^{*}
$$

y es inducido canónicamente por la traza $\pi_{*} \mathcal{O}_{Y} \rightarrow \mathcal{O}_{X}$ (véase [32, Thm.3.4.9]).

\subsection{Moduli de revestimientos y haces de línea.}

Sea $\mathcal{P} i c_{\tilde{\mathcal{Y}}_{\underline{n}}}$ el moduli de revestimientos finitos y separables de curvas lisas, $\pi: Y \rightarrow X$ (con $g_{X} \geq 2$ ), con haz de línea $L$ en $Y$ y sea $(X, Y, \pi, L)$ un punto racional.

Proposición 6.36. Se tiene un isomorfismo de espacios vectoriales:

$$
\mathrm{T}_{(X, Y, \pi, L)} \mathcal{P} i c_{\tilde{\mathcal{Y}}_{\underline{n}}} \simeq \mathrm{H}^{1}\left(Y, \mathcal{D}_{Y / k}^{1}(L, L)^{\pi}\right)
$$

donde $\mathcal{D}_{Y / k}^{1}(L, L)^{\pi}$ denota los operadores diferenciales en $\mathcal{D}_{Y / k}^{1}(L, L)$ tales que el morfismo símbolo valora en $\operatorname{Der}_{k}\left(\mathcal{O}_{Y}, \mathcal{O}_{Y}\right)^{\pi} \hookrightarrow \operatorname{Der}_{k}\left(\mathcal{O}_{Y}, \mathcal{O}_{Y}\right)$. 
Demostración. Se sigue del Teorema 6.12 y el Corolario 6.23.

Corolario 6.37. Se tiene una sucesión exacta de espacios vectoriales:

$$
0 \rightarrow \mathrm{H}^{1}\left(Y, \mathcal{O}_{Y}\right) \rightarrow \mathrm{H}^{1}\left(Y, \mathcal{D}_{Y / k}^{1}(L, L)^{\pi}\right) \rightarrow \mathrm{H}^{1}\left(X, \operatorname{Der}_{k}\left(\mathcal{O}_{Y}, \mathcal{O}_{Y}\right)^{\pi}\right) \rightarrow 0
$$

que se traduce geométricamente en:

$$
0 \rightarrow \mathrm{T}_{L} \operatorname{Jac}(Y) \rightarrow \mathrm{T}_{(X, Y, \pi, L)} \mathcal{P} i_{\tilde{\mathcal{Y}}_{\underline{n}}} \rightarrow \mathrm{T}_{(X, Y, \pi)} \mathcal{C}_{\text {ov }} \rightarrow 0
$$

Demostración. Se sigue de la Proposición 6.22 y la sucesión exacta de operadores diferenciales:

$$
0 \rightarrow \mathcal{O}_{Y} \rightarrow \mathcal{D}_{Y / k}^{1}(L, L)^{\pi} \rightarrow \operatorname{Der}_{k}\left(\mathcal{O}_{Y}, \mathcal{O}_{Y}\right)^{\pi} \rightarrow 0
$$

Denotemos $\mathcal{P} i c_{\tilde{\mathcal{Y}}_{X}}$ al moduli de revestimientos con haz de línea fijada la curva base $X$, y sea $(Y, \pi, L)$ un punto racional.

Corolario 6.38. Se tiene un isomorfismo de espacios vectoriales:

$$
\mathrm{T}_{(Y, \pi, L)} \mathcal{P}_{i \tilde{\mathcal{Y}}_{X}} \simeq \mathrm{H}^{0}\left(Y, \mathcal{O}_{R}\right)^{*} \oplus \mathrm{H}^{1}\left(Y, \mathcal{O}_{Y}\right)
$$

y una sucesión exacta:

$$
0 \rightarrow \mathrm{T}_{(Y, \pi, L)} \mathcal{P} i c_{\tilde{\mathcal{Y}}_{X}} \rightarrow \mathrm{T}_{(X, Y, \pi, L)} \mathcal{P} i c_{\tilde{\mathcal{Y}}_{\underline{n}}} \rightarrow \mathrm{T}_{X} \mathcal{M} \rightarrow 0
$$

Si además se fija la ramificación del revestimiento $\pi$, entonces:

$$
\mathrm{T}_{(Y, \pi, L)}{\mathcal{P} i \tilde{\mathcal{Y}}_{X}} \simeq \mathrm{H}^{1}\left(Y, \mathcal{O}_{Y}\right)
$$

Demostración. Se sigue de las Proposiciones 6.36 y 6.26

Denotemos $\mathcal{P} i c_{\tilde{\mathcal{Y}}_{\underline{\underline{n}}}}^{m}$ el moduli de revestimientos con haz de línea y estructuras de nivel y sea $\mathcal{Y}_{L}^{m}=\left(X, Y, \pi, x, z_{x}^{m}, \bar{y}, z_{\bar{y}}^{m}, L, \phi_{L}^{m}\right)$ un punto racional. Puede demostrarse que se tiene un isomorfismo de espacios vectoriales:

$$
\mathrm{T}_{\mathcal{Y}_{L}^{m}} \mathcal{P}_{i c_{\tilde{\mathcal{Y}}_{\underline{n}}}^{m}}^{m} \simeq \mathrm{H}^{1}\left(Y, \mathcal{D}_{Y / k}^{1}(L, L(-m \bar{y}))^{\pi}\right)
$$

y, si denotamos $\mathcal{P} i c_{\tilde{\mathcal{Y}}_{n}}^{\infty}$ al moduli de revestimientos con haz de línea y trivializaciones formales y tomamos un punto racional $\mathcal{Y}_{L}=\left(X, Y, \pi, x, z_{x}, \bar{y}, z_{\bar{y}}, L, \phi_{L}\right)$, se deduce:

$$
\mathrm{T}_{\mathcal{Y}_{L}} \mathcal{P} i c_{\tilde{\mathcal{Y}}_{\underline{n}}}^{\infty} \simeq \underset{m}{\lim _{m}} \mathrm{H}^{1}\left(Y, \mathcal{D}_{Y / k}^{1}(L, L(-m \bar{y}))^{\pi}\right)
$$

Antes de abordar el siguiente resultado (que proporciona un generador local para el espacio de moduli $\mathcal{P} i c_{\tilde{\mathcal{Y}}_{\underline{\underline{n}}}}^{\infty}$ ) recordemos que por la Proposición 1.28 , y sea cual sea la estructura de $k((z))$-álgebra que pongamos en $V$, se tiene que el grupo $\operatorname{SHeis}_{k((z))}^{V}$ (Definición 1.27 es una extensión de $\mathrm{G}_{k((z))}^{V}$ y $\mathrm{Heis}_{V}$. Por tanto, fijada una partición $\underline{n}$ y teniendo en cuenta que $\mathrm{G}_{\underline{\underline{n}}} \simeq \mathrm{G}_{\underline{n}, p}$ (y de modo análogo Heis $\operatorname{H}_{\underline{\underline{n}}} \simeq$ Heis $_{V_{\underline{n}, p}}$ ) se tiene que 
$\operatorname{SHeis}_{k((z))} \underset{V_{n}}{\longrightarrow} \operatorname{SHeis}_{k((z))} V_{n, p}$. Para abreviar, denotaremos a estos grupos SHeis $\underline{\underline{n}}$ y SHeis ${ }_{\underline{n}, p}$ respectivamente. Así mismo, si $k$ es un anillo, basta dotar a la categoría de esquemas de la topología étale para extender el resultado SHeis $_{\underline{n}} \simeq$ SHeis $_{\underline{n}, p}$ (véanse las Observaciones 1.11 y 6.30. Hechos estos comentarios, podremos centrar la demostración del siguiente resultado en el caso de puntos racionales.

Teorema 6.39. El grupo $\mathrm{SHeis}_{\underline{\underline{n}}}$ actúa en $\mathcal{P}_{i c_{\tilde{\mathcal{Y}}_{\underline{n}}}^{\infty}}$ y esta acción es localmente transitiva. Es decir, el grupo $\mathrm{SHeis}_{\underline{\underline{n}}}$ es un generador local del moduli $\mathcal{P} i c_{\tilde{\mathcal{Y}}_{\underline{\underline{n}}}^{\infty}}^{\infty}$.

Demostración. Recordemos que la imagen de $\mathcal{P} i c_{\tilde{\mathcal{Y}}_{n}}^{\infty}$ por el morfismo de Krichever, está definida por $\operatorname{los} W \in \mathcal{G} r\left(V_{\underline{n}}^{\text {univ }}\right)$ tales que:

$$
k \subset B_{W} \in \mathcal{G} \mathrm{r}\left(V_{\underline{\underline{n}}}^{\text {univ }}\right) \text { y } \operatorname{Tr}\left(B_{W}\right) \subset B_{W}
$$

(veáse el Teorema 5.20 y nótese que $W$ es $B_{W}$-módulo y $B_{W}$ verifica $B_{W} \cdot B_{W} \subseteq B_{W}$ ). Para ver que SHeis $\underline{\underline{n}}_{\underline{\underline{n}}}$ actúa en $\mathcal{P} i c_{\tilde{\mathcal{Y}}_{\underline{\underline{n}}}}^{\infty}$ hemos de comprobar si se verifican las siguientes condiciones para todo $\gamma \in$ SHeis $_{\underline{n}}$ :

- $\gamma(W) \in \mathcal{G} r\left(V_{\underline{n}}^{\text {univ }}\right)$.

- $B_{\gamma(W)} \in \mathcal{G r}\left(V_{\underline{n}}^{u n i v}\right)$.

- $\operatorname{Tr}\left(B_{\gamma(W)}\right) \subset B_{\gamma(W)}$.

Es fácil comprobar que $\operatorname{SHeis}_{\underline{n}}$ actúa en $\mathcal{G} \mathrm{r}\left(V_{\underline{n}}^{\text {univ }}\right)$, luego $\gamma(W) \in \mathcal{G} \mathrm{r}\left(V_{\underline{n}}^{\text {univ }}\right)$. Para el resto, teniendo en cuenta la Definición 1.27 de SHeis $\underline{\underline{n}}$, se deduce que:

$$
\begin{aligned}
B_{\gamma(W)}: & =\left\{f \in V_{S} \mid f \cdot \gamma(W) \subseteq W\right\}= \\
& =\left\{f \in V_{S} \mid \bar{g}^{-1}(f) \cdot W \subseteq W\right\}=\bar{g}^{-1}\left(B_{W}\right),
\end{aligned}
$$

donde $\bar{g} \in \mathrm{G}_{\underline{n}}$. Como $\mathrm{G}_{\underline{n}}$ actúa en $\mathcal{G} \mathrm{r}\left(V_{\underline{n}}^{\text {univ }}\right)$ se sigue que:

$$
B_{\gamma(W)}=\bar{g}^{-1}\left(B_{W}\right) \in \mathcal{G} \mathrm{r}\left(V_{\underline{n}}^{u n i v}\right) .
$$

La última de las condiciones se deduce de lo anterior y del Teorema 6.29.

Para ver que la acción es localmente transitiva basta ver que el morfismo órbita:

$$
\begin{aligned}
\text { SHeis }_{\underline{\underline{n}}} & \rightarrow \mathcal{P} i c_{\tilde{\mathcal{Y}}_{\underline{\underline{n}}}^{\infty}}^{\infty} \\
\gamma & \mapsto(W)
\end{aligned}
$$

es epiyectivo a nivel de espacios tangentes, esto es, si se verifica:

$$
\operatorname{LieSHeis}_{\underline{\underline{n}}} \rightarrow \mathrm{T}_{W} \mathcal{P} i c_{\tilde{\mathcal{Y}}_{\underline{\underline{n}}}}^{\infty} \rightarrow 0 .
$$


En efecto, basta tomar cohomología, $\lim _{\bar{m}}$ y $\varliminf_{m}$ en la sucesión:

$$
0 \rightarrow \mathcal{D}_{Y / k}^{1}\left(\mathcal{O}_{Y}, \mathcal{O}_{Y}(-m)\right)^{\pi} \rightarrow \mathcal{D}_{Y / k}^{1}\left(\mathcal{O}_{Y}, \mathcal{O}_{Y}(\bar{m})\right)^{\pi} \rightarrow \mathcal{D}_{Y / k}^{1}\left(\mathcal{O}_{Y}, \mathcal{O}_{Y}(\bar{m}) / \mathcal{O}_{Y}(-m)\right)^{\pi} \rightarrow 0
$$

y tener en cuenta que:

$$
\operatorname{Lie}\left(\operatorname{SHeis}_{\underline{n}}\right) \simeq \mathcal{D}_{V / k}^{1}(V, V)^{\pi} \underset{\vec{m}}{\simeq} \underset{m}{\lim _{m}} \lim _{Y / k}\left(\mathcal{O}_{Y}, \mathcal{O}_{Y}(\bar{m}) / \mathcal{O}_{Y}(-m)\right)^{\pi}
$$

donde el primer isomorfismo es la Proposición 2.14 y el segundo se deduce de C.6.

Observación 6.40. Pueden hacerse consideraciones análogas a las hechas en la Observación 6.30 si definimos el grupo SHeis ${ }_{\mathcal{O}_{\underline{\underline{n}}}}\left(V_{\underline{\underline{n}}}^{\text {univ }}((z))\right.$.

Calculamos ahora el espacio tangente a $\mathcal{P} i c_{\tilde{\mathcal{Y}}_{\underline{n}}^{\infty}}^{\infty}$ en función del espacio tangente a la Grassmanniana infinita relativa, en la que se sumerge vía el morfismo de Krichever.

Denotemos $D: \mathcal{P} i c_{\tilde{\mathcal{Y}}_{\underline{n}}}^{\infty} \rightarrow \mathbb{A}_{\underline{n}}$ el morfismo que manda cada punto $\mathcal{Y}_{L}$ de $\mathcal{P} i c_{\tilde{\mathcal{Y}}_{\underline{n}}}^{\infty}$ al polinomio $p(T)$ que define la estructura local del revestimiento (véase la Observación 5.18. Recordemos que podemos identificar un punto $W$ de $\mathcal{G} \mathrm{r}\left(V_{\underline{n}}^{\text {univ }}\right)$ con la pareja $\left(W_{p}, p(T)\right)$ de $\operatorname{Gr}\left(V_{\underline{n}, p}\right) \times \mathbb{A}_{\underline{n}}$.

Teorema 6.41. Sea $\mathcal{Y}_{L} \in \mathcal{P} i c_{\tilde{\mathcal{Y}}_{n}}^{\infty}$ un punto racional y $W$ su imagen por el morfismo de Krichever. Se tiene un isomorfismo de k-espacios vectoriales:

$$
\mathrm{T}_{\mathcal{Y}_{L}} \mathcal{P} i c_{\tilde{\mathcal{Y}}_{\underline{\underline{n}}}}^{\infty} \simeq \mathcal{D}_{B_{W_{p}} / k}^{1}\left(W_{p}, V_{\underline{\underline{n}}, p} / W_{p}\right)^{\operatorname{Tr}} \oplus \mathrm{T}_{p(T)} \operatorname{Im}(D)
$$

Demostración. Se demuestra de modo análogo al Teorema 6.34, teniendo en cuenta la Proposición 6.17 para calcular el tangente a la fibra de $D$ en $p(T)$.

Observación 6.42. Se tiene un diagrama:

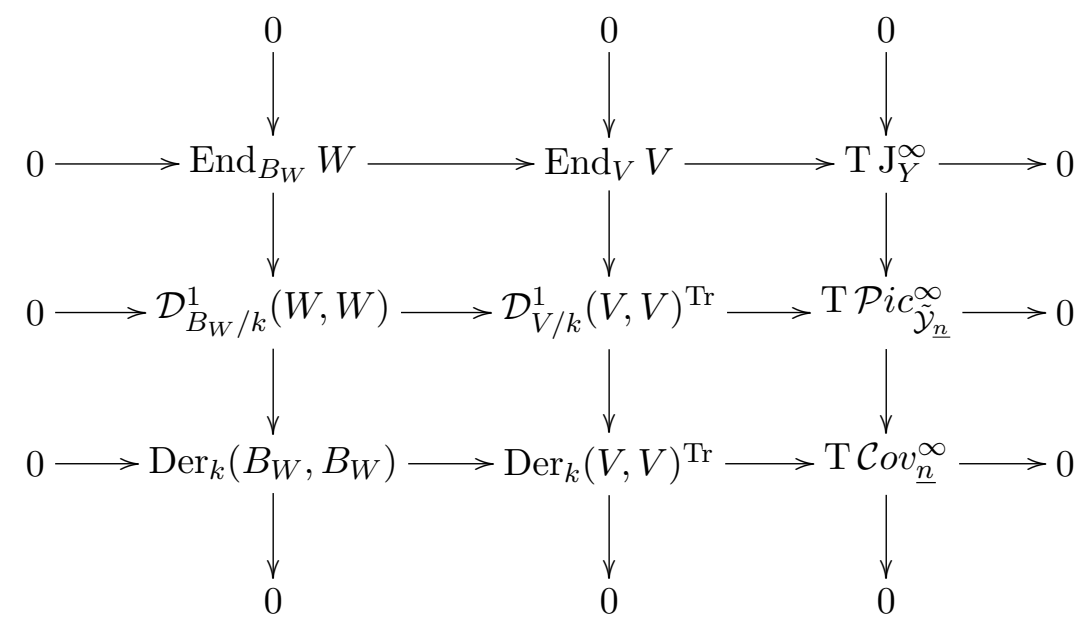




\subsection{Moduli de pares de Higgs.}

Sea $\mathcal{H i g g s}_{X}$ el moduli de pares de Higgs sobre $X$. Es conocido (véase por el ejemplo $[9$ Prop. 3.1.3]) que, si $(E, \varphi) \in \mathcal{H i g g s}_{X}(k)$, se tiene un isomorfismo de $k$-espacios vectoriales:

$$
\mathrm{T}_{(E, \varphi)} \mathcal{H i g g s}_{X} \simeq \mathbb{H}^{1}([, \varphi]),
$$

donde $[, \varphi]: \mathcal{E} \operatorname{nd}_{X} E \rightarrow \mathcal{E} \operatorname{nd}_{X} E \otimes \omega_{X}$ es el complejo definido por:

$$
[, \varphi](\eta):=[\eta, \varphi]=(\eta \otimes 1) \circ \varphi-\varphi \circ \eta .
$$

Otro modo de calcular este espacio tangente consiste en usar el morfismo de Hitchin:

$$
\begin{aligned}
H: \mathcal{H i g g s}_{X} & \rightarrow \mathcal{H}=\oplus_{i=1}^{n} \mathrm{H}^{0}\left(X, \omega_{X}^{\otimes i}\right) \\
(E, \varphi) & \mapsto \operatorname{ch}(\varphi),
\end{aligned}
$$

cuyas fibras están en correspondencia con la Jacobiana de la curva espectral $X_{\varphi}$. De este modo, puede verse que:

$$
\mathrm{T}_{(E, \varphi)} \mathcal{H i g g s}_{X} \simeq \mathrm{H}^{1}\left(X_{\varphi}, \mathcal{O}_{X_{\varphi}}\right) \oplus \mathrm{T}_{\operatorname{ch}(\varphi)} \mathcal{H}
$$

Además, dado que $\mathcal{H}$ es un espacio afín, se tiene que $\mathrm{T}_{\operatorname{ch}(\varphi)} \mathcal{H} \simeq \mathcal{H}$ y, esencialmente por dualidad de Serre, es $\mathcal{H}^{*} \simeq \mathrm{H}^{1}\left(X_{\varphi}, \mathcal{O}_{X_{\varphi}}\right)$ (véanse la ecuaciones (2.19) y (2.22) de [29]).

Teniendo en mente esta segunda caracterización, si tomamos el moduli $\mathcal{H i g g s}_{X}^{\infty}$ de pares de Higgs sobre $X$ con trivialización formal (Definición 4.1) y el morfismo de Hitchin formal definido en 4.8 :

$$
\mathcal{H}_{\infty}: \mathcal{H i g g s}_{X}^{\infty} \rightarrow \mathbb{A}
$$

(cuya imagen es $\mathcal{H}$ y la fibra en $p(T) \in \operatorname{Im}\left(\mathcal{H}_{\infty}\right)$ es isomorfa a la Jacobiana $\mathrm{Jac}_{X_{\varphi}}^{\infty}$ ), se tiene que, si $\mathcal{E}$ es un punto racional de $\mathcal{H}_{\text {igg }}^{\infty} s_{X}^{\infty}$, existe un isomorfismo de $k$-espacios vectoriales:

$$
\mathrm{T}_{\mathcal{E}} \mathcal{H i g g s}_{X}^{\infty} \simeq \underset{m}{\simeq} \lim _{m} \mathrm{H}^{1}\left(X_{\varphi}, \mathcal{O}_{X_{\varphi}}(-m)\right) \oplus \mathcal{H}
$$

(el cálculo del espacio tangente a $\mathrm{Jac}_{X_{\varphi}}^{\infty}$ es un caso particular del resultado (6.1.1)).

Interpretemos el espacio tangente a $\mathcal{H}$ igg $s_{X}^{\infty}$ dentro del espacio tangente a la Grassmanniana relativa, en la que se sumerge vía el morfismo de Krichever.

Sea $\mathcal{E}=(E, \varphi, \phi)$ un punto racional de $\mathcal{H} i g g s_{X}^{\infty}$, denotemos $E_{\varphi}$ al fibrado $E$ pensado como haz de línea en la curva espectral $X_{\varphi}$ y escribamos $\phi_{\varphi}$ para referirnos a su trivialización formal (véase la Proposición 4.18). Sea $W_{p}$ el punto racional de $\operatorname{Gr}\left(V^{u n i v}\right)$ que define $E_{\varphi}$ vía el morfismo de Krichever de la definición 4.3.4 Recordemos que se tiene un diagrama:

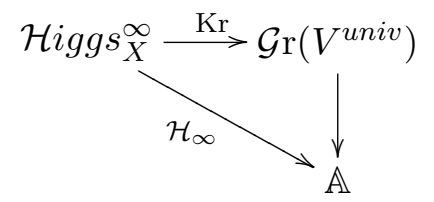


y un isomorfismo $\mathcal{G r}\left(V^{\text {univ }}\right) \simeq \operatorname{Gr}\left(k((z))^{n}\right) \times \mathbb{A}$. La fibra de $\mathcal{H}_{\infty}$ en un punto racional $p(T)$ de $\mathbb{A}$ es isomorfa a $\operatorname{Jac}_{X_{\varphi}}^{\infty}$, y por lo tanto, se tiene un isomorfismo de $k$-espacios vectoriales:

$$
\mathrm{T}_{\mathcal{E}} \mathcal{H} i g g s_{X}^{\infty} \simeq \mathrm{T}_{\left(E_{\varphi}, \phi_{\varphi}\right)} \operatorname{Jac}_{X_{\varphi}}^{\infty} \oplus \mathrm{T}_{p(T)} \operatorname{Im}\left(\mathcal{H}_{\infty}\right)
$$

Usando la Proposición 6.11, tenemos que el espacio tangente a $\operatorname{Jac}_{X_{\varphi}}^{\infty}$ (en términos del espacio tangente a la Grassmanniana) es isomorfo a:

$$
\operatorname{Hom}_{B_{W_{p}}}\left(W_{p}, V_{p} / W_{p}\right),
$$

donde $B_{W_{p}}$ denota el álgebra estabilizadora de $W_{p}$ en $V_{p}$ y corresponde al punto de la Grassmanniana que define la curva espectral $X_{\varphi}$.

En definitiva, se tiene un isomorfismo de $k$-espacios vectoriales:

$$
\mathrm{T}_{\mathcal{E}} \mathcal{H i g g s}_{X}^{\infty} \simeq \operatorname{Hom}_{B_{W_{p}}}\left(W_{p}, V_{p} / W_{p}\right) \oplus \mathcal{H}
$$

Observación 6.43. Puede también calcularse el espacio tangente a $\mathcal{H} i g g s_{X}^{\infty}$ en términos de hipercohomología como sigue. Denotamos $\mathcal{H}_{\text {iggs }}^{m}$ el moduli de triples $\left(E, \varphi, \phi_{m}\right)$ donde $(E, \varphi) \in \mathcal{H i g g s}_{X}$ y $\phi_{m}$ es una estructura de nivel de $E$ de orden $m$ compatible con el campo de Higgs en el sentido de que el siguiente diagrama es conmutativo:

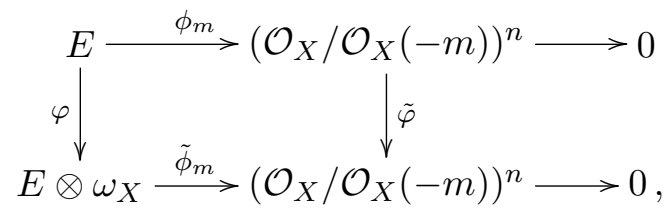

donde $\tilde{\phi}_{m}$ es la estructura de nivel inducida (recordemos que al fijar $\left(X, x, t_{m}\right)$ tenemos de manera natural una estructural de nivel en $\omega_{X}$ ) y $\tilde{\varphi}$ consiste en multiplicar por la matriz de orden $n$ cuyas entradas son $b_{i+1, i}=1, b_{i, n}=-a_{i}=-(-1)^{i} \operatorname{Tr}\left(\Lambda^{i} \varphi\right)$ y 0 en otro caso.

A partir del diagrama de compatibilidad del campo de Higgs con la estructura de nivel construimos el siguiente cuadro:

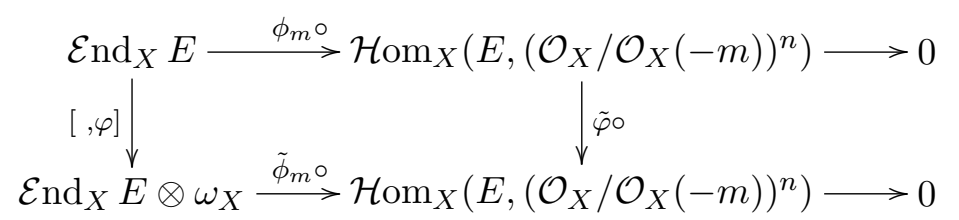

(que ya no tiene por qué ser conmutativo) y definimos el siguiente complejo de haces en $X$ :

$$
\begin{aligned}
K^{m}: \mathcal{E} \operatorname{nd}_{X} E & \rightarrow \mathcal{H o m}_{X}\left(E,\left(\mathcal{O}_{X} / \mathcal{O}_{X}(-m)\right)^{n}\right) \\
\eta & \mapsto \tilde{\varphi} \circ \phi_{m} \circ \eta+\tilde{\phi}_{m} \circ[\eta, \varphi] .
\end{aligned}
$$

Su primer grupo de hipercohomología, $\mathbb{H}^{1}\left(K^{m}\right)$, viene dado por parejas:

$$
\left(\left\{\eta_{i j}\right\},\left\{\gamma_{i}\right\}\right) \in C^{1}\left(\mathcal{U}, \mathcal{E} \operatorname{nd}_{X} E\right) \oplus C^{0}\left(\mathcal{U}, \mathcal{H o m}_{X}\left(E,\left(\mathcal{O}_{X} / \mathcal{O}_{X}(-m)\right)^{n}\right)\right)
$$


tales que $\eta_{i j}$ es un 1-cociclo y:

$$
\tilde{\varphi} \circ \phi_{m} \circ \eta_{i j}+\tilde{\phi}_{m} \circ\left[\eta_{i j}, \varphi\right]=-\left(\gamma_{j}-\gamma_{i}\right)_{\mid U_{i j}}
$$

en $C^{1}\left(\mathcal{U}, \mathcal{H}_{X}\left(E,\left(\mathcal{O}_{X} / \mathcal{O}_{X}(-m)\right)^{n}\right)\right)$. Puede demostrarse que, si $\left(E, \varphi, \phi_{m}\right) \in \mathcal{H i g g s}_{X}^{m}(k)$, se tiene un isomorfismo de $k$-espacios vectoriales:

$$
\mathrm{T}_{\left(E, \varphi, \phi_{m}\right)} \mathcal{H i g g s}_{X}^{m} \simeq \mathbb{H}^{1}\left(K^{m}\right) .
$$

Dado que $\mathcal{H}_{i g g s_{X}^{\infty}}^{\infty}=\varliminf_{\varliminf_{m}} \mathcal{H} i g g s_{X}^{m}$, si $\mathcal{E}$ es un punto racional de $\mathcal{H} i g g s_{X}^{\infty}$, se deduce que:

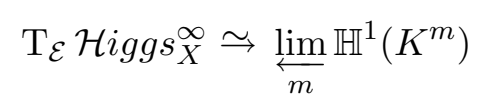




\section{Capítulo 7}

\section{Ecuaciones del moduli de pares de Higgs.}

En este capítulo damos las ecuaciones del moduli de pares de Higgs con trivialización formal. Antes de ello, adaptaremos algunos de los resultados acerca de funciones Tau y funciones de Baker descritos en [3, 33, 31], en los que se basa el cálculo de las ecuaciones en cuestión.

\subsection{Geometría formal.}

Trabajaremos sobre un cuerpo $k$ algebraicamente cerrado de característica nula. Como en capítulos anteriores, dado un punto racional $p(T) \in \mathbb{A}$, denotaremos:

$$
V_{p}=k((z))[T] / p(T) \quad V_{p}^{+}=k[[z]][T] / p(T) .
$$

A partir de ahora supondremos que se tiene la siguiente descomposición de $V_{p}$ como $k((z))$ álgebra separable:

$$
V_{p} \simeq V_{1} \times \cdots \times V_{r}
$$

donde $V_{i}=k((z))\left[T_{i}\right] / T_{i}^{n_{i}}-z u_{i}\left(T_{i}\right), n_{1}+\cdots+n_{r}=n \mathrm{y} u_{i}\left(T_{i}\right)$ son invertibles en $k\left[\left[T_{i}\right]\right]$. Además, es fácil ver que se tiene un isomorfismo:

$$
k\left(\left(T_{i}\right)\right) \simeq k((z))\left[T_{i}\right] / T_{i}^{n_{i}}-z u_{i}\left(T_{i}\right)
$$

donde $z$ pensada como serie en $T_{i}$ vale $T_{i}^{n_{i}} u_{i}\left(T_{i}\right)^{-1}$.

Esta elección viene motivada por el siguiente hecho geométrico:

Proposición 7.1. Sea $(E, \varphi, \phi)$ un punto racional de $\mathcal{H i g g s}_{X}^{\infty}$, y sea $\pi: X_{\varphi} \rightarrow X$ el revestimiento espectral asociado. Supongamos que $\pi^{-1}(x)=\sum_{i=1}^{r} n_{i} y_{i}$, donde $\sum_{i_{1}}^{r} n_{i}=n e$ $y_{i} \neq y_{j}$ para todo $i \neq j$. Entonces se tiene que:

$$
\widehat{\mathcal{O}}_{X_{\varphi}, \pi^{-1}(x)} \simeq\left(k[[z]]\left[T_{1}\right] / T_{1}^{n_{1}}-z u_{1}\left(T_{1}\right)\right) \times \cdots \times\left(k[[z]]\left[T_{r}\right] / T_{r}^{n_{r}}-z u_{r}\left(T_{r}\right)\right),
$$


donde $u_{i}\left(T_{i}\right) \in k[[z]]\left[T_{i}\right]$.

Demostración. Se sigue de la Proposición 5.3 y la Observación 5.4.

\subsubsection{Jacobiana formal y morfismo de Abel.}

En virtud de los isomorfismos de la ecuación (7.1.1) y la sección 1.1, el functor de invertibles $\underline{V}_{p}^{*}$ es representable por un $k$-esquema formal en grupos cuya componente conexa del origen descompone como:

$$
\Gamma_{V_{p}}=\Gamma_{V_{p}}^{-} \times \mathbb{G}_{m}^{r} \times \Gamma_{V_{p}}^{+}
$$

donde, si tenemos en cuenta la descomposición de $V_{p}$ en $k$-álgebras irreducibles $V_{i}$, resulta:

$$
\Gamma_{V_{p}}^{ \pm} \simeq \Gamma_{V_{1}}^{ \pm} \times \cdots \times \Gamma_{V_{r}}^{ \pm}
$$

Además, la Jacobiana formal de la curva espectral formal $\widehat{X}_{V}, \mathcal{J}\left(\widehat{X}_{V}\right)$, es isomorfa a $\Gamma_{V_{p}}^{-}$(véase [3, Teorema 4.14] y también [31]).

Sea $\widehat{\mathbb{A}}_{\infty}$ el esquema formal en grupos:

$$
\widehat{\mathbb{A}}_{\infty}:=\underset{n}{\lim } \operatorname{Spec} k\left[\left[t_{1}, \ldots, t_{n}\right]\right]
$$

dotado de la ley de grupo aditiva. Definimos:

$$
k\left\{\left\{t_{1}, t_{2}, \ldots\right\}\right\}:={\underset{\varliminf}{n}}_{n} k\left[\left[t_{1}, \ldots, t_{n}\right]\right]
$$

como el anillo $\mathcal{O}_{\widehat{\mathbb{A}}_{\infty}}$, y escribiremos:

$$
\mathbb{A}_{\infty}^{r}:=\widehat{\mathbb{A}}_{\infty} \times \stackrel{r}{.} \times \widehat{\mathbb{A}}_{\infty} .
$$

Como estamos suponiendo que la característica de $k$ es cero, la aplicación exponencial es el isomorfismo de $k$-esquemas formales en grupos ([3, Definición 4.11]):

$$
\begin{aligned}
\exp : \widehat{\mathbb{A}}_{\infty}^{r} & \rightarrow \mathcal{J}\left(\widehat{X}_{V}\right) \\
\left(\left\{a_{i}^{(1)}\right\}_{i>0}, \ldots,\left\{a_{i}^{(r)}\right\}_{i>0}\right) & \mapsto\left(\exp \left(\sum_{i>0} \frac{a_{i}^{(1)}}{T_{1}^{i}}\right), \ldots, \exp \left(\sum_{i>0} \frac{a_{i}^{(r)}}{T_{r}^{i}}\right)\right)
\end{aligned}
$$

Por lo tanto, identificaremos el grupo $\mathcal{J}\left(\widehat{X}_{V}\right)$ con el espectro formal del anillo:

$$
k\left\{\left\{t_{1}^{(1)}, \ldots\right\}\right\} \hat{\otimes} \cdots \hat{\otimes} k\left\{\left\{t_{1}^{(r)}, \ldots\right\}\right\}
$$

y su elemento universal será:

$$
v=\prod_{i=1}^{r} \exp \left(\sum_{j \geq 1} \frac{t_{j}^{(i)}}{T_{i}^{j}}\right),
$$


El morfismo de Abel de grado uno ([31]):

$$
\phi_{1}: \widehat{X}_{V} \rightarrow \mathcal{J}\left(\widehat{X}_{V}\right)
$$

es el correspondiente a la $r$-upla de series:

$$
\left(\left(1-\frac{\bar{T}_{1}}{T_{1}}\right)^{-1}, \ldots,\left(1-\frac{\bar{T}_{r}}{T_{r}}\right)^{-1}\right)
$$

donde estamos distinguiendo las variables:

$$
\bar{T}_{i} \in \mathcal{O}_{\widehat{X}_{V}}=k\left[\left[\bar{T}_{1}\right]\right] \times \cdots \times k\left[\left[\bar{T}_{r}\right]\right] \simeq V_{p}^{+}
$$

y las variables $T_{i} \in \mathcal{J}\left(\widehat{X}_{V}\right)$.

Equivalentemente, el morfismo de Abel es el inducido por el morfismo de anillos:

$$
\begin{aligned}
k\left\{\left\{t_{1}^{(1)}, t_{2}^{(1)}, \ldots\right\}\right\} \hat{\otimes} \cdots \hat{\otimes} k\left\{\left\{t_{1}^{(r)}, t_{2}^{(r)}, \ldots\right\}\right\} & \rightarrow k\left[\left[\bar{T}_{1}\right]\right] \times \cdots \times k\left[\left[\bar{T}_{r}\right]\right] \\
t_{i}^{(j)} & \mapsto \bar{T}_{j}^{(i)}
\end{aligned}
$$

\subsubsection{Funciones Tau.}

En esta sección recordamos la definición y propiedades de las funciones Tau de puntos de Grassmannianas infinitas dadas en [3, 31]. Estas funciones hacen las veces de funciones Theta de la Jacobiana dentro del contexto de la Geometría formal.

Consideremos la fibración de Grassmannianas infinitas:

$$
\operatorname{Gr}\left(k((z))^{n}\right) \times \mathbb{A} \rightarrow \mathbb{A}
$$

y sea $p(T)$ un punto racional de $\mathbb{A}$. La fibra en $p(T)$ es $\operatorname{Gr}\left(V_{p}\right)$, donde $V_{p}=k((z))[T] / p(T)$.

Consideremos la acción por homotecias de $\Gamma_{V_{p}}$ en $\operatorname{Gr}\left(V_{p}\right)$ :

$$
\mu: \Gamma_{V_{p}} \times \operatorname{Gr}\left(V_{p}\right) \rightarrow \operatorname{Gr}\left(V_{p}\right)
$$

y definamos un haz de Poincaré en $\Gamma_{V_{p}} \times \operatorname{Gr}\left(V_{p}\right)$ como:

$$
\mathcal{P}:=\mu^{*} \operatorname{Det}_{V_{p}}^{*},
$$

(donde Det $V_{p}^{*}$ es el dual del haz determinante definido en [3]). Para cada punto racional $W \in \operatorname{Gr}\left(V_{p}\right)$, se tiene un haz de línea sobre $\Gamma_{V_{p}}$ dado por:

$$
\widetilde{\mathcal{L}}_{\tau}(W):=\mathcal{P}_{\mid \Gamma_{V_{p}} \times\{W\}}
$$

y un morfismo natural:

$$
\mathrm{H}^{0}\left(\Gamma_{V_{p}} \times \operatorname{Gr}\left(V_{p}\right), \mathcal{P}\right) \rightarrow \mathrm{H}^{0}\left(\Gamma_{V_{p}} \times\{W\}, \widetilde{\mathcal{L}}_{\tau}(W)\right) .
$$


Definición 7.2. Llamaremos sección Tau del punto $W$, que denotaremos $\tilde{\tau}_{W}$, a la imagen de $\mu^{*} \Omega_{+}$por el morfismo 7 7.1.2 , donde $\Omega_{+}$es la sección global canónica de Det ${ }_{V_{p}}^{*}$ definida en [3, Definición 3.14].

Nótese que $\tilde{\tau}_{W}$ no se puede interpretar como una verdadera función sobre $\Gamma_{V_{p}} \times\{W\}$, pues $\widetilde{\mathcal{L}}_{\tau}(W)$ no es en general trivial. El análogo algebraico de la función Tau definida por Segal y Wilson ([42]), se obtiene restringiendo $\widetilde{\mathcal{L}}_{\tau}(W)$ al subgrupo formal $\Gamma_{V_{p}}^{-} \simeq \mathcal{J}\left(\widehat{X}_{V}\right) \subset$ $\Gamma_{V_{p}}$. Detallemos un poco más esta última afirmación:

Definimos $\mathcal{L}_{\tau}(W):=\widetilde{\mathcal{L}}_{\tau}(W)_{\mid \Gamma_{V_{p}}^{-} \times\{W\}}$, que es trivial sobre $\Gamma_{V_{p}}^{-} \times\{W\}$ (véase [3]). La siguiente sección global se encarga de darnos una trivialización de dicho haz:

$$
\sigma_{0}\left(g_{\bullet}\right):=g_{\bullet} \cdot \rho_{W},
$$

siendo $\rho_{W}$ un elemento no nulo en la fibra de $\mathcal{L}_{\tau}(W)$ sobre el punto $(1, W) \in \Gamma_{V_{p}}^{-} \times\{W\}$. La función Tau se define como la restricción de $\tilde{\tau}_{W}$ a $\Gamma_{V_{p}}^{-}$.

Definición 7.3. Se define la función Tau del punto $W, \tau_{W}$, como la función:

$$
\tau_{W} \in \mathcal{O}_{\Gamma_{V_{p}}^{-}} \simeq \mathcal{O}_{\mathcal{J}\left(\widehat{X}_{V}\right)} \simeq k\left\{\left\{t_{1}^{(1)}, \ldots\right\}\right\} \hat{\otimes} \cdots \hat{\otimes} k\left\{\left\{t_{1}^{(r)}, \ldots\right\}\right\}
$$

dada por:

$$
\tau_{W}\left(g_{\bullet}\right)=\frac{\tilde{\tau}_{W}\left(g_{\bullet}\right)}{\sigma_{0}\left(g_{\bullet}\right)}=\frac{\mu^{*} \Omega_{+}\left(g_{\bullet}\right)}{\sigma_{0}\left(g_{\bullet}\right)}=\frac{\Omega_{+}\left(g_{\bullet} \cdot W\right)}{g_{\bullet} \cdot \rho_{W}}
$$

para $g_{\bullet}=\prod_{i=1}^{r} \exp \left(\sum_{j \geq 1} \frac{t_{j}^{(i)}}{T_{i}^{j}}\right) \in \mathcal{J}\left(\widehat{X}_{V}\right) \simeq \Gamma_{V_{p}}^{-}$.

Observación 7.4. Cuando estamos trabajando sobre la curva formal base $\widehat{X}=\operatorname{Spf} k[[z]]$, si $\Omega \in \operatorname{Gr}(k((z)))$, su función $\tau$ sobre $\mathcal{J}(\widehat{X}) \simeq \Gamma^{-} \simeq$ Spf $k\left\{\left\{t_{1}, \ldots\right\}\right\}$ vale ([[3]):

$$
\tau_{\Omega}(g)=\frac{\tilde{\tau}_{\Omega}(g)}{\sigma_{0}(g)}=\frac{\mu^{*} \Omega_{+}(g)}{\sigma_{0}(g)}=\frac{\Omega_{+}(g W)}{g \rho_{W}}
$$

para $g=\exp \left(\sum_{j \geq 1} \frac{t_{j}}{z^{j}}\right) \in \mathcal{J}(\widehat{X}) \simeq \Gamma^{-}$.

\subsubsection{Funciones de Baker-Akhiezer.}

Consideremos la composición:

$$
\tilde{\beta}: \widehat{X}_{V} \times \Gamma_{V_{p}} \times \operatorname{Gr}\left(V_{p}\right) \stackrel{\phi_{1} \times \operatorname{Id}}{\rightarrow} \Gamma_{V_{p}} \times \Gamma_{V_{p}} \times \operatorname{Gr}\left(V_{p}\right) \stackrel{m \times \operatorname{Id}}{\longrightarrow} \Gamma_{V_{p}} \times \operatorname{Gr}\left(V_{p}\right)
$$

donde $\phi_{1}: \widehat{X}_{V} \rightarrow \Gamma_{V_{p}}$ es el morfismo de Abel de grado uno (con valores en $\Gamma_{V_{p}}^{-} \simeq \mathcal{J}\left(\widehat{X}_{V}\right) \subset$ $\Gamma_{V_{p}}$ ) y $m$ es la ley de grupo en $\Gamma_{V_{p}}$. Dado un punto racional $W \in \operatorname{Gr}\left(V_{p}\right)$, denotaremos $\tilde{\beta}_{W}$ a la restricción de $\tilde{\beta}$ a $\widehat{X}_{V} \times \Gamma_{V_{p}} \times\{W\}$. 
Definición 7.5. La sección Baker-Akhiezer (BA) de $W \in \operatorname{Gr}\left(V_{p}\right)$ es $\tilde{\psi}_{W}:=v^{-1} \cdot \beta_{W}^{*}\left(\tilde{\tau}_{W}\right)$, donde:

$$
\beta_{W}^{*}: \mathrm{H}^{0}\left(\Gamma_{V_{p}} \times\{W\}, \widetilde{\mathcal{L}}_{\tau}(W)\right) \rightarrow \mathrm{H}^{0}\left(\widetilde{X}_{V} \times \Gamma_{V_{p}} \times\{W\}, \tilde{\beta}_{W}^{*} \widetilde{\mathcal{L}}_{\tau}(W)\right)
$$

es el morfismo inducido por $\tilde{\beta}_{W}^{*}$. Es decir, la estamos normalizando de modo que:

$$
{\tilde{\psi_{W}}}_{\mid x_{0}^{(i)} \times \Gamma_{V_{p}}}=v_{i}^{-1},
$$

donde $x_{0}^{(i)}$ es el origen de $\widehat{X}_{V_{i}}$ y $v$ es $v_{i}$ en la componente $\widehat{X}_{V_{i}}$ de $\widehat{X}_{V}$.

La función BA de un punto $W \in \operatorname{Gr}\left(V_{p}\right)$ se define por la fórmula:

$$
\psi_{W}\left(T_{\bullet}, g_{\bullet}\right)=v^{-1} \frac{\tau_{W}\left(g_{\bullet} \cdot \phi_{1}\left(z_{\bullet}\right)\right)}{\tau_{W}\left(g_{\bullet}\right)}
$$

donde $T_{\bullet}=\left(T_{1}, \ldots, T_{r}\right)$ y $g_{\bullet} \in \Gamma_{V_{p}}^{-}$.

Para dar una expresión explícita de esta función, identificamos la Jacobiana formal $\mathcal{J}\left(\widehat{X}_{V}\right)$ con $\widehat{\mathbb{A}}_{\infty}^{r}$ (vía el isomorfismo exponencial) y usamos el morfismo de Abel:

$$
\widehat{X}_{V} \stackrel{\phi_{1}}{\rightarrow} \mathcal{J}\left(\widehat{X}_{V}\right),
$$

que manda $T_{j}$ al punto de $\mathcal{J}\left(\widehat{X}_{V}\right)$ con coordenadas:

$$
\left[T_{j}\right]:=\left((0, \ldots), \ldots,\left(T_{j}, \frac{T_{j}^{2}}{2}, \frac{T_{j}^{3}}{3}, \ldots\right), \ldots,(0, \ldots)\right)
$$

o, equivalentemente, la aplicación está inducida por el siguiente morfismo de anillos:

$$
\begin{aligned}
k\left\{\left\{t_{1}^{(1)}, \ldots\right\}\right\} \hat{\otimes} \cdots \hat{\otimes} k\left\{\left\{t_{1}^{(r)}, \ldots\right\}\right\} & \rightarrow k\left[\left[T_{1}\right]\right] \times \cdots \times k\left[\left[T_{r}\right]\right] \\
t_{i}^{(j)} & \mapsto\left(0, \ldots, 0, \frac{T_{j}^{(i)}}{i}, 0, \ldots, 0\right) .
\end{aligned}
$$

Es claro que se tiene un morfismo suma:

$$
\begin{aligned}
\widehat{X}_{V} \times \mathcal{J}\left(\widehat{X}_{V}\right) & \rightarrow \mathcal{J}\left(\widehat{X}_{V}\right) \\
\left(T_{\bullet}, t_{\bullet}\right) & \mapsto t_{\bullet}+\left[T_{\bullet}\right]
\end{aligned}
$$

donde $T_{\bullet}=\left(T_{1}, \ldots, T_{r}\right), t_{\bullet}=\left(t^{(1)}, \ldots, t^{(r)}\right)$ y $t_{\bullet}+\left[T_{\bullet}\right]$ denota el punto de $\mathcal{J}\left(\widehat{X}_{V}\right)$ con coordenadas $\left(\ldots, t_{i}^{(j)}+\frac{T_{j}^{i}}{i}, \ldots\right)$.

De este modo, definimos la función BA de un punto $W \in \operatorname{Gr}\left(V_{p}\right)$ (en característica cero) por la fórmula $([33,31])$ :

$$
\psi_{W}\left(T_{\bullet}, t_{\bullet}\right)=\prod_{i=1}^{r} \exp \left(-\sum_{j \geq 1} \frac{t_{j}^{(i)}}{T_{i}^{j}}\right) \cdot\left(\frac{\tau_{W}\left(t_{\bullet}+\left[T_{\bullet}\right]\right)}{\tau_{W}\left(t_{\bullet}\right)}\right)
$$


Observación 7.6. Si $\Omega \in \operatorname{Gr}(k((z)))$, su función de Baker en característica cero es ([3, 33]):

$$
\psi_{\Omega}(z, t)=\exp \left(-\sum_{j \geq 1} \frac{t_{j}}{z^{j}}\right) \cdot\left(\frac{\tau_{\Omega}(t+[z])}{\tau_{\Omega}(t)}\right)
$$

Observación 7.7. Si bien cuando trabajamos con un punto $\Omega \in \operatorname{Gr}(k((z)))$ la función Tau genera el subespacio $\Omega$ de $k((z))$ ([33]), no es cierto que la función Tau de un punto $W \in$ $\operatorname{Gr}\left(V_{p}\right)$ genere $W$. Con el propósito de arreglar este problema se da la siguiente definición en [31] y [26].

Recordemos la descomposición $V_{p} \simeq V_{1} \times \cdots \times V_{r}$.

Definición 7.8. La $u$-ésima función BA de un punto $W \in \operatorname{Gr}\left(V_{p}\right)$ es la función:

$$
\psi_{u, W}\left(T_{\bullet}, t_{\bullet}\right):=\left(\xi_{u 1} \exp \left(-\sum_{j \geq 1} \frac{t_{j}^{(1)}}{T_{1}^{j}}\right) \frac{\tau_{W_{u 1}}\left(t_{\bullet}+\left[T_{1}\right]\right)}{\tau_{W}\left(t_{\bullet}\right)}, \ldots, \xi_{u r} \exp \left(-\sum_{j \geq 1} \frac{t_{j}^{(n)}}{T_{r}^{j}}\right) \frac{\tau_{W_{u r}}\left(t_{\bullet}+\left[T_{r}\right]\right)}{\tau_{W}\left(t_{\bullet}\right)}\right)
$$

donde

- $1 \leq u \leq r$.

- $W_{u v}:=\left(1, \ldots, T_{u}, \ldots, T_{v}^{-1}, \ldots, 1\right) \cdot W$.

- $t_{\bullet}+\left[T_{v}\right]:=\left(t^{(1)}, \ldots, t^{(v)}+\left[T_{v}\right], \ldots, t^{(n)}\right)$.

- $\xi_{u i}$ vale -1 si $i>u$ y 1 si $i \leq u$.

El siguiente teorema afirma que $W$ está generado (como subespacio infinito de $V_{p}$ ) por la funciones $u$-ésimas de BA.

Teorema 7.9. [31 Theorem 3.6] Sea $W \in \mathrm{Gr}^{0}\left(V_{p}\right)$. Entonces:

$$
\psi_{u, W}\left(T_{\bullet}, t_{\bullet}\right)=\left(1, \ldots, T_{u}, \ldots, 1\right) \cdot \sum_{i>0}\left(\psi_{u, W}^{i, 1}\left(T_{1}\right), \ldots, \psi_{u, W}^{i, r}\left(T_{r}\right)\right) p_{u i, W}\left(t_{\bullet}\right)
$$

donde $\left\{\left(\psi_{u, W}^{i, 1}\left(T_{1}\right), \ldots, \psi_{u, W}^{i, r}\left(T_{r}\right)\right) \mid i>0,1 \leq u \leq n\right\}$ es una base de $W y p_{u i, W}\left(t_{\bullet}\right)$ son funciones en $t_{\text {. }}$.

Observación 7.10. Recordemos que el superíndice 0 en $\operatorname{Gr}^{0}\left(V_{p}\right)$ denota que estamos en la componente conexa de donde la función índice de $W$ toma valor 0 (véase la Observación 3.7.

Observación 7.11. Para estudiar la componente conexa de índice $m(m>0), \operatorname{Gr}^{m}\left(V_{p}\right)$, necesitamos elegir un elemento $v_{m}$ tal que $\operatorname{dim}_{k} V_{p}^{+} / v_{m} V_{p}^{+}=m$ con el fin de conseguir un isomorfismo $\mathrm{Gr}^{m}\left(V_{p}\right) \simeq \operatorname{Gr}^{0}\left(V_{p}\right)$ que permita definir la sección global $\Omega_{+}^{m}$ del dual del haz determinante de $\operatorname{Gr}^{m}\left(V_{p}\right)$ como la imagen de la sección canónica $\Omega_{+}$de $\operatorname{Det}_{V_{p}}^{*}$ (véase [3] y [33, Remark 4]). Tomamos $v_{m}$ como sigue: 
- para $m \leq \frac{1}{2}(r-n)$, sean $p, q, s, t$ números enteros definidos por $-m=q \cdot(n-r)+p$, $0 \leq p<n-r, p=s \cdot r+t, 0 \leq t<r$. Tomamos:

$$
v_{m}:=\left(z^{-1} \cdot T_{\bullet}\right)^{q} T_{1}^{s+1} \cdots T_{t}^{s+1} T_{t+1}^{s} \cdots T_{r}^{s}
$$

- para $m>\frac{1}{2}(r-n)$, elegimos:

$$
v_{m}:=\left(z^{-1} \cdot T_{\bullet}\right) \cdot v_{r-n-m}^{-1}
$$

Teorema 7.12. [31. Theorem 3.7] Sea $W \in \mathrm{Gr}^{m}\left(V_{p}\right)$. Se tiene que:

$$
\psi_{u, W}\left(T_{\bullet}, t_{\bullet}\right)=v_{m}^{-1}\left(1, \ldots, T_{u}, \ldots, 1\right) \cdot \sum_{i>0}\left(\psi_{u, W}^{(i, 1)}\left(T_{1}\right), \ldots, \psi_{u, W}^{(i, r)}\left(T_{r}\right)\right) p_{u i, W}\left(t_{\bullet}\right)
$$

donde $\left\{\left(\psi_{u, W}^{(i, 1)}\left(T_{1}\right), \ldots, \psi_{u, W}^{(i, r)}\left(T_{t}\right)\right) \mid i>0,1 \leq u \leq t\right\}$ es una base de $W$ y $p_{u i, W}\left(t_{\bullet}\right)$ son funciones en $t_{\text {. }}$.

En particular, un elemento de $V_{p}$ yace en $W$ si y sólo si se puede expresar como una combinación lineal de:

$$
\psi_{1, W}\left(T_{\bullet}, t_{\bullet}\right), \ldots, \psi_{r, W}\left(T_{\bullet}, t_{\bullet}\right)
$$

para ciertos valores de los parámetros t.

Observación 7.13. Si $\Omega \in \operatorname{Gr}^{s}(k((z)))$ entonces ([33]):

$$
\psi_{\Omega}(z, t)=z^{1-s} \sum_{i>0} \psi_{\Omega}^{(i)}(z) p_{i}(t)
$$

donde $\left\{\psi_{\Omega}^{(i)}(z)\right\}_{i}$ es una base de $\Omega$ y $p_{i}(t)$ funciones en $t$. En particular, si $\Omega$ es el punto definido por $\omega_{X}$ entonces $s=g-1$, siendo $g$ el género de la curva lisa $X$. Si denotamos $\Omega^{-1}$ al punto definido por $\omega_{X}^{-1}$, entonces $s=1-3 g$.

\section{Función de Baker adjunta.}

Dado que $V_{p}$ es una $k((z))$-álgebra separable, la métrica de la traza:

$$
\operatorname{Tr}: V_{p} \times V_{p} \rightarrow k((z)),
$$

es no degenerada. Con esto, definimos el siguiente pairing:

$$
\begin{aligned}
\mathrm{T}_{2}: V_{p} \times V_{p} & \rightarrow k \\
(a, b) & \mapsto \operatorname{Res}_{z=0}(\operatorname{Tr}(a, b)) d z .
\end{aligned}
$$

Lema 7.14. 33] 31] El pairing $\mathrm{T}_{2}$ induce un isomorfismo de k-esquemas:

$$
\begin{aligned}
R: \operatorname{Gr}\left(V_{p}\right) & \rightarrow \operatorname{Gr}\left(V_{p}\right) \\
W & \mapsto W^{\perp},
\end{aligned}
$$

donde $W^{\perp}$ denota el ortogonal a $W$ respecto de $\mathrm{T}_{2}$. 
Se tienen las siguientes propiedades:

- $R\left(\mathrm{Gr}^{m}\left(V_{p}\right)\right)=\mathrm{Gr}^{-m}\left(V_{p}\right)$.

- $R^{*} \operatorname{Det}_{V_{p}} \simeq \operatorname{Det}_{V_{p}}$

- $(g \cdot W)^{\perp}=g^{-1} \cdot W^{\perp}$, para $W \in \operatorname{Gr}\left(V_{p}\right)$ y $g \in \mathcal{J}\left(\widehat{X}_{V}\right)$.

- $R^{*} \Omega_{+}^{m}=\Omega_{+}^{-m}$.

- $\tau_{W^{\perp}}(g)=\tau_{W}\left(g^{-1}\right)$.

- $W_{u v}^{\perp}=\left(W^{\perp}\right)_{v u}$

Definición 7.15. Se define la $u$-ésima función adjunta de Baker-Akhiezer de un punto $W \in$ $\operatorname{Gr}\left(V_{p}\right)$ por:

$$
\psi_{u, W}^{*}\left(T_{\bullet}, t_{\bullet}\right):=\psi_{u, W^{\perp}}\left(T_{\bullet},-t_{\bullet}\right)
$$

Explítamente:

$$
\begin{aligned}
\psi_{u, W}^{*}\left(T_{\bullet}, t_{\bullet}\right) & :=\left(\xi_{u 1} \exp \left(\sum_{j \geq 1} \frac{t_{j}^{(1)}}{T_{1}^{j}}\right) \frac{\tau_{W_{u 1}^{\perp}}\left(-t_{\bullet}+\left[T_{1}\right]\right)}{\tau_{W}^{\perp}\left(-t_{\bullet}\right)}, \ldots, \xi_{u n} \exp \left(\sum_{j \geq 1} \frac{t_{j}^{(n)}}{T_{n}^{j}}\right) \frac{\tau_{W_{u r}^{\perp}}\left(-t_{\bullet}+\left[T_{r}\right]\right)}{\tau_{W}^{\perp}\left(-t_{\bullet}\right)}\right)= \\
& =\left(\xi_{u 1} \exp \left(\sum_{j \geq 1} \frac{t_{j}^{(1)}}{T_{1}^{j}}\right) \frac{\tau_{W_{1 u}}\left(t_{\bullet}-\left[T_{1}\right]\right)}{\tau_{W}\left(t_{\bullet}\right)}, \ldots, \xi_{u r} \exp \left(\sum_{j \geq 1} \frac{t_{j}^{(n)}}{T_{r}^{j}}\right) \frac{\tau_{W_{r u}}\left(t_{\bullet}-\left[T_{r}\right]\right)}{\tau_{W}\left(t_{\bullet}\right)}\right)
\end{aligned}
$$

Teorema 7.16. Sea $W \in \mathrm{Gr}^{m}\left(V_{p}\right)$. Se tiene que:

$$
\psi_{u, W}^{*}\left(T_{\bullet}, t_{\bullet}\right)=v_{r-n-m}^{-1}\left(1, \ldots, T_{u}, \ldots, 1\right) \cdot \sum_{i>0}\left(\psi_{u, W}^{*(i, 1)}\left(T_{1}\right), \ldots, \psi_{u, W}^{*(i, r)}\left(T_{r}\right)\right) p_{u i, W}^{*}\left(t_{\bullet}\right)
$$

donde $\left\{\left(\psi_{u, W}^{*(i, 1)}\left(T_{1}\right), \ldots, \psi_{u, W}^{*(i, r)}\left(T_{r}\right)\right) \mid i>0,1 \leq u \leq r\right\}$ es una base de $W^{\perp} y p_{u i, W}^{*}\left(t_{\bullet}\right)$ son funciones en $t$.

En particular, un elemento de $V_{p}$ yace en $W^{\perp}$ si y sólo si se puede expresar como una combinación lineal de:

$$
\psi_{1, W}^{*}\left(T_{\bullet}, t_{\bullet}\right), \ldots, \psi_{r, W}^{*}\left(T_{\bullet}, t_{\bullet}\right)
$$

para ciertos valores de los parámetros t.

\subsubsection{Producto de funciones de Baker-Akhiezer.}

Sean $\Omega \in \operatorname{Gr}(k((z)))$ y $W \in \operatorname{Gr}\left(V_{p}\right)$ puntos racionales. Consideremos sus funciones BA:

$$
\psi_{\Omega}(z, t) \quad \psi_{W}\left(T_{\bullet}, t_{\bullet}\right),
$$

que viven en $\widehat{X} \times \Gamma^{-}$y $\widehat{X}_{V} \times \Gamma_{V_{p}}^{-}$respectivamente. 
La gráfica del morfismo $\widehat{\pi}: \widehat{X}_{V} \rightarrow \widehat{X}$ induce una aplicación:

$$
f: \widehat{X}_{V} \times \Gamma_{V_{p}}^{-} \times \Gamma^{-} \rightarrow\left(\widehat{X}_{V} \times \Gamma_{V_{p}}^{-}\right) \times\left(\widehat{X} \times \Gamma^{-}\right) .
$$

Observación 7.17. Obsérvese que el morfismo gráfica es el inducido por el morfismo de anillos:

$$
\begin{aligned}
k[[z]] \otimes_{k}\left(k\left[\left[T_{1}\right]\right] \times \cdots \times k\left[\left[T_{r}\right]\right]\right) & \rightarrow k\left[\left[T_{1}\right]\right] \times \cdots \times k\left[\left[T_{r}\right]\right] \\
z \otimes_{k}\left(T_{1}, \ldots, T_{r}\right) & \mapsto\left(z T_{1}, \ldots, z T_{r}\right)
\end{aligned}
$$

Esta expresión tiene sentido gracias a la ecuación (7.1.1), que expresa $z$ como serie en $T_{i}$.

Definición 7.18. Se define el producto de funciones BA de los puntos $\Omega \in \operatorname{Gr}(k((z)))$ y $W \in \operatorname{Gr}\left(V_{p}\right)$ por la fórmula:

$$
\psi_{W}\left(T_{\bullet}, t_{\bullet}\right) * \psi_{\Omega}(z, t):=f^{*}\left(\psi_{W}\left(T_{\bullet}, t_{\bullet}\right) \otimes_{k} \psi_{\Omega}(z, t)\right)
$$

De modo análogo se define el producto de $\psi_{\Omega}(z, t)$ por la función $u$-ésima de BA de $W$, $\psi_{u, W}\left(T_{\bullet}, t_{\bullet}\right)$.

Proposición 7.19. Sean $W \in \operatorname{Gr}\left(V_{p}\right)$ y $\Omega \in \operatorname{Gr}(k((z)))$ tales que $\Omega \cdot W \in \operatorname{Gr}\left(V_{p}\right)$. Entonces:

$$
\left\{\psi_{\Omega}(z, t) * \psi_{1, W}\left(T_{\bullet}, t_{\bullet}\right), \ldots, \psi_{\Omega}(z, t) * \psi_{r, W}\left(T_{\bullet}, t_{\bullet}\right)\right\}
$$

son un sistema de generadores de $\Omega \cdot W$, y se tiene:

$$
\psi_{\Omega}(z, t) * \psi_{u, W}\left(T_{\bullet}, t_{\bullet}\right)=\left(\psi_{\Omega}(z, t) \cdot \psi_{u, W}^{(1)}\left(T_{1}, t_{\bullet}\right), \ldots, \psi_{\Omega}(z, t) \cdot \psi_{u, W}^{(r)}\left(T_{r}, t_{\bullet}\right)\right),
$$

donde:

$$
\begin{gathered}
\psi_{\Omega}(z, t)=\exp \left(-\sum_{j \geq 1} \frac{t_{j}}{z^{j}}\right) \cdot\left(\frac{\tau_{\Omega}(t+[z])}{\tau_{\Omega}(t)}\right) \\
\psi_{u, W}^{(i)}=\xi_{u i} \exp \left(-\sum_{j \geq 1} \frac{t_{j}^{(i)}}{T_{i}^{j}}\right) \frac{\tau_{W_{u i}}\left(t_{\bullet}+\left[T_{i}\right]\right)}{\tau_{W}\left(t_{\bullet}\right)} .
\end{gathered}
$$

Demostración. Son un sistema de generadores por el Teorema7.12 y la Observación 7.13 . La fórmula explícita se sigue de la Observación 7.6 de la Definición 7.8 y de la definición del morfismo gráfica.

\subsection{Ecuaciones del moduli.}

Recordemos que el Teorema 4.15 dice que un punto racional $(W, p(T)) \in \mathcal{U}_{X}^{\infty} \times \mathbb{A}$ está en la imagen del morfismo:

$$
\operatorname{Higgs}_{X}^{\infty} \hookrightarrow \mathcal{U}_{X}^{\infty} \times \mathbb{A}
$$


si y sólo si $T(W) \subseteq W \cdot \Omega$, donde $\Omega \in \operatorname{Gr}(k((z)))$ es el punto que define el haz dualizante $\omega_{X}$ vía el morfismo de Krichever, y $T$ es la homotecia multiplicar por $T$ en $V_{p}$. Teniendo en cuenta la descomposición $V_{p} \simeq V_{1} \times \cdots \times V_{r}$, la homotecia multiplicar por $T$ en $V_{p}$ se traduce en la homotecia multiplicar por $T_{\bullet}=\left(T_{1}, \ldots, T_{r}\right)$ y por lo tanto, si pensamos $T$ como operador en $V_{p}$, es fácil deducir que $T$ es autoadjunto respecto del pairing $\mathrm{T}_{2}$ definido en la sección 7.1.3, esto es:

$$
\mathrm{T}_{2}\left(T_{\bullet} \cdot v, v^{\prime}\right)=\mathrm{T}_{2}\left(v, T_{\bullet} \cdot v^{\prime}\right) \quad \forall v, v^{\prime} \in V_{p} \simeq V_{1} \times \cdots V_{r} .
$$

Teorema 7.20. Sea $(W, p(T)) \in \mathcal{U}_{X}^{\infty} \times \mathbb{A}$ un punto racional, $\Omega \in \mathrm{Gr}^{g-1}(k((z)))$ y supongamos que $W \in \mathrm{Gr}^{m}\left(V_{p}\right)\left(\right.$ con $\left.m \neq \frac{1}{2}(r-n)\right)$.

$(W, p(T))$ está en la imagen de $\mathcal{H}_{i g g} s_{X}^{\infty} \hookrightarrow \mathcal{U}_{X}^{\infty} \times \mathbb{A}$ si y sólo si:

$$
\mathrm{T}_{2}\left(\frac{v_{r-n-m} \cdot T_{\bullet} \cdot \psi_{u, W}^{*}\left(T_{\bullet}, t_{\bullet}\right)}{\left(1, \ldots, T_{u}, \ldots, 1\right)}, \frac{v_{m} \cdot \psi_{\Omega^{-1}}(z, t) \cdot \psi_{v, W}\left(T_{\bullet}, t_{\bullet}\right)}{z^{3 g} \cdot\left(1, \ldots, T_{v}, \ldots 1\right)}\right)=0 \quad 1 \leq u, v \leq r .
$$

Demostración. Por el teorema 4.15, $(W, p(T)) \in \mathcal{U}_{X}^{\infty} \times \mathbb{A}$ está en la imagen de $\mathcal{H}_{i g g s_{X}^{\infty} \hookrightarrow} \hookrightarrow$ $\mathcal{U}_{X}^{\infty} \times \mathbb{A}$ si y sólo si $T \cdot W \subseteq W \cdot \Omega$. Ahora bien:

$$
T \cdot W \subseteq W \cdot \Omega \Longleftrightarrow W \cdot \Omega^{-1} \subseteq T^{-1} \cdot W \Longleftrightarrow\left(T^{-1} \cdot W\right)^{\perp} \subseteq\left(W \cdot \Omega^{-1}\right)^{\perp},
$$

donde $\perp$ denota el ortogonal con respecto a $\mathrm{T}_{2}$.

Por ser $T$ autoadjunto, se tiene que:

$$
\begin{aligned}
\left(T^{-1} \cdot W\right)^{\perp}: & =\left\{v \in V_{p} \mid \mathrm{T}_{2}\left(v, T^{-1} \cdot w\right)=0 \quad \forall w \in W\right\}= \\
& =\left\{v \in V_{p} \mid \mathrm{T}_{2}\left(T^{-1} \cdot v, w\right)=0 \quad \forall w \in W\right\}= \\
& =\left\{v \in V_{p} \mid T^{-1} \cdot v \in W^{\perp}\right\}=T \cdot W^{\perp} .
\end{aligned}
$$

Como multiplicar por $T$ equivale a multiplicar por $T_{\bullet}$, usando el Teorema 7.16 se tiene que:

$$
\psi_{1, W}^{*}\left(T_{\bullet}, t_{\bullet}\right), \ldots, \psi_{r, W}^{*}\left(T_{\bullet}, t_{\bullet}\right)
$$

son un sistema de generadores para $W^{\perp}$. Por lo tanto:

$$
T_{\bullet} \cdot \psi_{1, W}^{*}\left(T_{\bullet}, t_{\bullet}\right), \ldots, T_{\bullet} \cdot \psi_{r, W}^{*}\left(T_{\bullet}, t_{\bullet}\right)
$$

son un sistema de generadores de $T \cdot W^{\perp}$ y (por el Teorema 7.16):

$T_{\bullet} \cdot \psi_{u, W}^{*}\left(T_{\bullet}, t_{\bullet}\right)=v_{r-n-m}^{-1}\left(1, \ldots, T_{u}, \ldots, 1\right) \cdot \sum_{i>0}\left(T_{1} \cdot \psi_{u, W}^{*(i, 1)}\left(T_{1}\right), \ldots, T_{r} \cdot \psi_{u, W}^{*(i, r)}\left(T_{r}\right)\right) p_{u i, W}^{*}\left(t_{\bullet}\right)$.

Teniendo en cuenta que $\mathrm{T}_{2}$ es no degenerada, la Observación 7.13 y los Teoremas 7.12 y 7.16, se concluye que $T \cdot W^{\perp} \subseteq\left(\Omega^{-1} \cdot W\right)^{\perp}$ si y sólo si se verifican las siguientes ecuaciones:

$$
\mathrm{T}_{2}\left(\frac{v_{r-n-m} \cdot T_{\bullet} \cdot \psi_{u, W}^{*}\left(T_{\bullet}, t_{\bullet}\right)}{\left(1, \ldots, T_{u}, \ldots, 1\right)}, \frac{v_{m} \cdot \psi_{\Omega^{-1}}(z, t) \cdot \psi_{v, W}\left(T_{\bullet}, t_{\bullet}\right)}{z^{3 g} \cdot\left(1, \ldots, T_{v}, \ldots 1\right)}\right)=0 \quad 1 \leq u, v \leq r .
$$




\subsubsection{Ecuaciones para polinomios irreducibles.}

Describimos ahora las ecuaciones de $\mathcal{H}_{\text {iggs }}^{\infty}{ }_{X}^{\infty}$ en el caso en que el revestimiento espectral $\pi: X_{\varphi} \rightarrow X$ está totalmente ramificado en $x \in X$ (esto es, $\pi^{-1}(x)=n y$ ). Calculamos esta ecuaciones en términos de los coeficientes característicos del campo de Higgs formal.

Asumir que el revestimiento espectral está totalmente ramificado en $x \in X$ implica que el polinomio característico del campo de Higgs formal es irreducible. Luego, a partir de ahora, supondremos que tenemos un polinomio irreducible:

$$
p(T)=T^{n}-a_{1} T^{n-1}+\cdots+(-1)^{n} a_{n}
$$

La multiplicación por $T$ en $V_{p}=k((z))[T] / p(T)$ se expresa, con respecto a la base $\left\{1, T, \ldots, T^{n-1}\right\}$, como la matriz:

$$
T=\left(\begin{array}{cccccc}
0 & & & & 0 & (-1)^{n+1} a_{n} \\
1 & \cdot & & & \cdot & (-1)^{n} a_{n-1} \\
\cdot & 1 & \cdot & & \cdot & \cdot \\
\cdot & & \cdot & \cdot & \cdot & \cdot \\
\cdot & & & \cdot & 0 & -a_{2} \\
0 & \cdot & & 0 & 1 & a_{1}
\end{array}\right)
$$

En virtud de la ecuación (7.1.1), estamos en el siguiente caso:

$$
V_{p}=k((z))[T] / p(T) \simeq k((z))[T] / T^{n}-z u(T) \simeq k((T)),
$$

donde $u(T)$ puede calcularse explícitamente en términos de $a_{1}, \ldots, a_{n}$. Nótese además que multiplicar por $T^{-1}$ tiene sentido en $V_{p}$ (pero no en $V_{p}^{+}$).

Si $W \in \operatorname{Gr}^{m}\left(V_{p}\right)$ entonces su función BA está dada por la fórmula:

$$
\psi_{W}\left(T, t_{n}\right)=T^{1-m} \sum_{i>0} \psi_{W}^{(i)}(T) p_{i}\left(t_{n}\right)
$$

donde $\left\{\psi_{W}^{(i)}(T)\right\}_{i}$ es una base de $W$ y $p_{i}\left(t_{n}\right)$ son funciones en $t_{n}$ (véase la Observación 7.13 y nótese que estamos escribiendo $t_{n}$ para las coordenadas de $\left.\Gamma_{V_{p}}\right)$. Además, el ortogonal $W^{\perp}$ a $W$ (respecto de la métrica $\mathrm{T}_{2}$ definida en la sección 7.1.3 yace en $\mathrm{Gr}^{-m}\left(V_{p}\right)$. Por lo tanto:

$$
\psi_{W}^{*}\left(T, t_{n}\right)=T^{1+m} \sum_{i>0} \psi_{W}^{*,(i)}(T) p_{i}^{*}\left(t_{n}\right)
$$

donde $\left\{\psi_{W}^{*,(i)}(T)\right\}_{i}$ es una base de $W^{\perp}$ y $p_{i}^{*}\left(t_{n}\right)$ son funciones en $t_{n}$.

Además, como en la sección 7.1.4 podemos definir el producto de la función BA $\Psi_{\Omega^{-1}}(z, t)$ de $\Omega^{-1} \in \operatorname{Gr}(k((z)))$ por la función BA $\Psi_{W}\left(T, t_{n}\right)$ de $W \in \operatorname{Gr}\left(V_{p}\right)$ teniendo en sólo en cuenta que ahora la estructura de $k((z))$-álgebra de $V_{p} \simeq k((T))$ está dada por:

$$
\begin{aligned}
k((z)) & \rightarrow k((T)) \\
z & \mapsto T^{n} u(T)^{-1}
\end{aligned}
$$


y que el morfismo gráfica es el inducido por:

$$
\begin{aligned}
k[[z]] \otimes_{k} k[[T]] & \rightarrow k[[T]] \\
z \otimes_{k} T & \mapsto z T
\end{aligned}
$$

Para calcular explícitamente las ecuaciones de $\mathcal{H} i g g s_{X}^{\infty}$, pensaremos las funciones de BA meramente como funciones en $V_{p}$, esto es, como polinomios en $T$ con coeficientes en $k((z))$. De este modo, podemos escribir:

$$
\begin{array}{ll}
\Psi_{W}(T)=\sum_{i=0}^{n-1} \Psi_{W, i}(z) T^{i}, & \Psi_{W, i}(z) \in k((z)) \\
\Psi_{W}^{*}(T)=\sum_{i=0}^{n-1} \Psi_{W, i}^{*}(z) T^{i}, & \Psi_{W, i}^{*}(z) \in k((z))
\end{array}
$$

Sea $(E, \varphi, \phi)$ un punto racional de $\mathcal{H} i g g s_{X}^{\infty} \mathrm{y}$

$$
p_{\widehat{\varphi}}(T)=T^{n}-a_{1} T^{n-1}+\cdots+(-1)^{n} a_{n} \in \mathbb{A}
$$

su polinomio característico formal. La fórmulas de Newton-Girard (véase [25, I.2]) expresan $\operatorname{Tr}\left(T^{k}\right)$ en función de los coeficientes $a_{i}=\operatorname{Tr}\left(\Lambda^{i} T\right)$ como sigue:

$$
\operatorname{Tr}\left(T^{j}\right)=\left|\begin{array}{cccccc}
a_{1} & 1 & 0 & \cdot & \cdot & \cdot \\
2 a_{2} & a_{1} & 1 & 0 & \cdot & \cdot \\
\cdot & \cdot & \cdot & \cdot & \cdot & \cdot \\
\cdot & \cdot & & \cdot & \\
\cdot & \cdot & & & \cdot \\
j a_{j} & a_{j-1} & \cdot & \cdot & \cdot & a_{1}
\end{array}\right| \in k((z)), \quad \forall j \geq 0
$$

donde $a_{j}=0$ para $j>n$.

Denotemos $\mathcal{H i g g s}_{X}^{\infty}(n)$ al subesquema de $\mathcal{H}_{i g g s_{X}^{\infty}}^{\infty}$ en el que el revestimiento espectral está totalmente ramificado $\left(\pi^{-1}(x)=n y\right)$.

Teorema 7.21. Sea $(W, p(T))$ un punto racional de $\mathcal{U}_{X}^{\infty} \times \mathbb{A}$. Entonces, $(W, p(T))$ yace en la imagen de $\mathcal{H i g g s}_{X}^{\infty}(n) \hookrightarrow \mathcal{U}_{X}^{\infty} \times \mathbb{A}$ si y sólo si $p(T)$ es irreducible y:

$$
\sum_{k=0}^{2 n-2} \sum_{i+j=k} \operatorname{Res}_{z=0}\left(\Psi_{W, i}^{*}(z) \Psi_{\Omega^{-1}}(z) \Psi_{W, j}(z) \operatorname{Tr}\left(T^{k-1}\right)\right) \frac{d z}{z^{3 g}}=0,
$$

donde $\operatorname{Tr}\left(T^{k-1}\right)$ se expresa en función de los coeficientes de $p(T)$ por la ecuación (7.2.5).

Demostración. En efecto, usando las expresiones (7.2.1), (7.2.2), la Observación 7.13 y el Teorema 7.20, obtenemos que $(W, p(T))$ está en la imagen de $\mathcal{H} i g g s_{X}^{\infty}(n) \hookrightarrow \mathcal{U}_{X}^{\infty} \times \mathbb{A}$ si y sólo si:

$$
\mathrm{T}_{2}\left(T \cdot T^{-1+m} \cdot \psi_{W}^{*}\left(T, t_{n}\right), T^{-1-m} \cdot \psi_{\Omega^{-1}}(z, t) \cdot \psi_{W}\left(T, t_{n}\right) \cdot z^{-3 g}\right)=0
$$


Para hacer los cálculos, observemos que la estructura de $k$-álgebra de $V_{p}$ no es sino la multiplicación estandar de polinomios módulo $p(T)$. Luego utilizando las expresiones polinómicas 7.2.3), 7.2.4 y la definición de $\mathrm{T}_{2}$ (sección 7.1.3), la ecuación 7.2.6) se convierte en:

$$
\operatorname{Res}_{z=0}\left(\operatorname{Tr}\left(\sum_{k=0}^{2 n-2} \sum_{i+j=k} \Psi_{W, i}^{*}(z) \Psi_{\Omega^{-1}}(z) \Psi_{W, j}(z) T^{k-1} z^{-3 g}\right)\right) d z=0
$$

Teniendo en cuenta que la aplicación traza es $k((z))$-lineal y la linealidad del residuo se deduce el resultado.

Observación 7.22. Esta técnicas pueden usarse también para calcular las ecuaciones del subesquema $\mathcal{H}_{i g g s_{X}^{\infty}}^{\infty}(1, \ldots, 1)$ de $\mathcal{H} i g g s_{X}^{\infty}$ en el que el revestimiento espectral es no ramificado, que es el caso estudiado en [23]. 


\section{Parte IV}

\section{Apéndices.}





\section{Apéndice A}

\section{Grassmanniana Infinita.}

Sea $k$ un cuerpo y $V$ un $k$-espacio vectorial. Nos proponemos construir un $k$-esquema, que llamaremos Grassmanniana de $V$, cuyos puntos racionales sean precisamente los subespacios vectoriales discretos de $V$ (definición A.5). Para ello definiremos su functor de puntos (definición A.13) y demostraremos que es representable en la categoría de $k$-esquemas (teorema A.20]. Todos los resultados de este apéndice están en [2], [35] y [3].

Lo primero que tenemos que saber es cómo trabajar con subespacios de $V$ de dimensión infinita. La siguiente definición da una manera de comparar tales subespacios afirmando que dos ellos son equivalentes cuando su "diferencia" es algo finito.

Definición A.1. Dados dos subespacios $A$ y $B$ de $V$, diremos que son conmensurables, y lo denotaremos por $A \sim B$, cuando $A+B / A \cap B$ sea un $k$-espacio vectorial de dimensión finita.

Se verifica que las sumas finitas e intersecciones finitas de conmensurables (con un subespacio fijado) son conmensurables (con dicho subespacio).

Sea $V$ un $k$-espacio vectorial, definimos el conjunto de subespacios $\tau$ tales que:

- Si $A, B \in \tau$ entonces $A \sim B$.

- Si $A, B \in \tau$ entonces $A+B$ y $A \cap B \in \tau$.

- $\cap_{B \in \tau} B=(0)$.

- $V=\lim _{B \in \tau} V / B$.

- Dado $A \in \tau$, la topología inducida en $V / A$ contiene a todos los subespacios de dimensión finita.

(nos referiremos a ellas como las condiciones de $\tau$ ). Esto dota a $V$ de una estructura de $k$-espacio vectorial linealmente topológico, donde dichos subespacios forman una base de entornos de (0), respecto de la cual es separado (tercera condición) y completo (cuarta condición). Si $A$ es un subespacio de $V$, la topología inducida en $A$ es la definida por la base 
de entornos $\{A \cap B: B \in \tau\}$; la inducida en $V / A$ es la definida por la base de entornos $\{A+B / A: B \in \tau\}$.

Recuerdese que la completación de $V$ con respecto a $\tau$ es $\widehat{V}=\varliminf_{\varliminf_{B \in \tau}} V / B$. Análogamente, si $A \subseteq V$ es $\widehat{A}=\varliminf_{B \in \tau} A / A \cap B$ y $\widehat{V / A}=\varliminf_{B \in \tau} V / A+B$. Se dice que $V$ es completo cuando el morfismo canónico $V \rightarrow \widehat{V}$ es isomorfismo.

Observación A.2. Dada una sucesión exacta:

$$
0 \rightarrow B \rightarrow V \rightarrow V / B \rightarrow 0
$$

la sucesión exacta entre los completados respecto $\tau$ es exacta, pues los morfismos del sistema proyectivo de $B$ son epiyectivos.

Proposición A.3. Para cualquier $A \subseteq V$ se tiene un isomorfismo canónico $\widehat{A} / A \simeq \widehat{V} / V$. En particular, $V$ es completo si y sólo si un subespacio $A \subseteq V$ (y por tanto todos) es completo.

Demostración. Fijado $A \in V$ tenemos el diagrama:

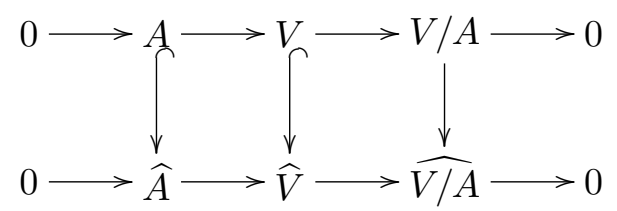

Donde las inclusiones son consecuencia de que la topología es separada. Como:

$$
\widehat{V / A}=\lim _{\overparen{B \in \tau}} V / A+B=V / A
$$

aplicando el lema de la serpiente se concluye.

\section{Ejemplo A.4.}

1. Sea $V$ un $k$-espacio vectorial y $\tau$ el conjunto de todos los subespacios de dimensión finita, entonces se verifican todas las condiciones de $\tau$.

2. Sea $V=k((z))$ y $\tau$ el conjunto de todos los subespacios conmensurables con $V^{+}=$ $k[[z]]$. Se verifican todas las condiciones excepto la completitud.

3. Sea $V=k((z))$ y $\tau$ el conjunto de todos los subespacios conmensurables con el subespacio $V^{+}=k[[z]]$ tales que contienen a $z^{n} k[[z]]$ para algún $n \in \mathbb{Z}$. Se verifican todas las condiciones.

Fijemos un subespacio $V^{+} \in \tau$. A partir de ahora consideraremos $\left(V, \tau, V^{+}\right)$. 
Definición A.5. Un subespacio vectorial $L$ de $V$ se dice discreto si $L \cap V^{+}$y $V / L+V^{+}$ son $k$-espacios vectoriales de dimensión finita. Nótese que no depende la elección de $V^{+}$, sólo de su clase de conmesura.

Definimos entonces la Grassmanniana de $V$, como puro conjunto, por:

$$
\operatorname{Gr}(V, \tau)=\{\text { Subespacios discretos de } V\}
$$

Dotemos a este conjunto de una estructura de esquema sobre $k$. Sea $S$ un $k$-esquema y $(V, \tau)$ como antes, el $\mathcal{O}_{S}$-módulo:

$$
V_{S}:=V \otimes_{k} \mathcal{O}_{S}
$$

tiene una topología natural definida por la base de entornos:

$$
\left\{A_{S} \text { tales que } A \in \tau\right\}
$$

Y definimos su completación (primero se cambia de base y luego se completa) por:

$$
\widehat{V_{S}}=\lim _{\overleftarrow{A \in \tau}}\left(V / A \otimes_{k} \mathcal{O}_{S}\right)
$$

Si $B$ es un subespacio de $V$ se sigue:

$$
\widehat{B_{S}}=\lim _{\overparen{A \in \tau}}\left((B / B \cap A) \otimes_{k} \mathcal{O}_{S}\right) \quad\left(\widehat{V / B)_{S}}=\lim _{\overparen{A \in \tau}}\left((V / A+B) \otimes_{k} \mathcal{O}_{S}\right)\right.
$$

Observación A.6. El morfismo $(\widehat{B})_{S} \rightarrow \widehat{B_{S}}$ no es en general un isomorfismo. Un caso en el que sí lo es, es cuando $S$ es el espectro de una $k$-álgebra finita.

Tenemos ahora una generalización de la proposición A.3.

Proposición A.7. Si $B \in \tau$ entonces:

$$
(\widehat{V / B})_{S}=\widehat{V_{S}} / \widehat{B_{S}}=\left(V / B \otimes_{k} \mathcal{O}_{S}\right)
$$

con lo que $\widehat{V_{S}} / \widehat{B_{S}}$ es un haz de $\mathcal{O}_{S}$-módulos cuasicoherente.

Demostración. Es $V_{S} \rightarrow \widehat{V_{S}}$ inyectiva y $V_{S} \rightarrow V / B \otimes_{k} \mathcal{O}_{S}$ epiyectiva. Además sabemos que $(\widehat{V / B})_{S}=(V / B)_{S}=V / B \otimes_{k} \mathcal{O}_{S}$. Luego de la siguiente sucesión exacta se deduce el enunciado:

$$
0 \rightarrow \widehat{B_{S}} \rightarrow \widehat{V_{S}} \rightarrow(\widehat{V / B})_{S} \rightarrow 0
$$

Para generalizar la noción de subespacio discreto necesitamos en primer lugar que, si $L \subseteq \widehat{V_{S}}$ sea $L_{S^{\prime}} \subseteq \widehat{V_{S^{\prime}}}$ para todo morfismo de $k$-esquemas $S^{\prime} \rightarrow S$ (pues queremos que la definición sea functorial, estable por cambios de base). Esta condición equivale a que $\widehat{V_{S}} / L$ sea plano sobre $S$. Antes de dar la definición de submódulo discreto empezamos dando una serie de resultados previos. 
Lema A.8. Sea:

$$
0 \rightarrow L^{\prime} \rightarrow L \rightarrow M \rightarrow 0
$$

una sucesión exacta de módulos sobre un anillo local $\mathcal{O}$, donde $L^{\prime}$ y $L$ son libres de tipo finito y verifican que para cada $s \in S=\operatorname{Spec} \mathcal{O}$ se tiene $L_{s}^{\prime} \hookrightarrow L_{s}\left(L_{s}=L \otimes_{\mathcal{O}} \kappa(s)\right)$. Entonces $M$ es libre de tipo finito.

Demostración. $M$ es el conúcleo de dos libres de tipo finito, luego es de presentación finita (y por tanto de tipo finito). Como $L_{s}^{\prime} \hookrightarrow L_{s}$ entonces $\operatorname{Tor}^{i}(M, \kappa(s))=0$, luego $M$ es plano, y por ser de tipo finito es libre, ya que $\mathcal{O}$ es un anillo local.

Sea $L$ un sub- $\mathcal{O}_{S}$-módulo de $\widehat{V_{S}}$ y $A, B \in \tau$ tales que $A \subset B \subset V$. Para demostrar las siguientes proposiciones haremos uso del siguiente diagrama:

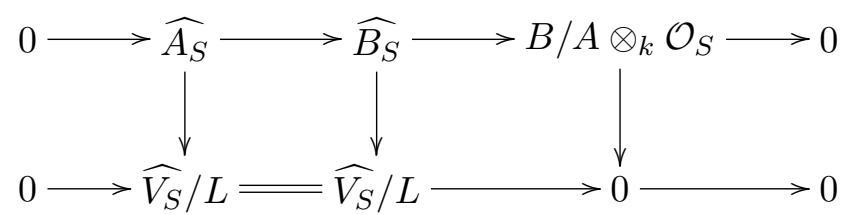

Aplicando el lema de la serpiente se deduce la siguiente sucesión exacta:

$$
O \rightarrow L \cap \widehat{A_{S}} \rightarrow L \cap \widehat{B_{S}} \rightarrow(B / A)_{S} \rightarrow \widehat{V_{S}} / L+\widehat{A_{S}} \rightarrow \widehat{V_{S}} / L+\widehat{B_{S}} \rightarrow 0
$$

Proposición A.9. Sea $L$ un submódulo cuasicoherente de $\widehat{V_{S}}$ tal que $\widehat{V_{S}} / L$ es plano y supongamos que existe $A \in \tau$ tal que $L \cap \widehat{A_{S}}$ localmente libre de tipo finito y $\widehat{V_{S}} / L+\widehat{A_{S}}=0$. Se verifica:

1. Para todo $s \in S$ existe un entorno abierto $U$ de s en $S$ tal que $\left(L \cap \widehat{A_{S}}\right)_{U}=L_{U} \cap \widehat{A_{U}}$.

2. Si $B$ es un subespacio de $V$ tal que $A \subset B$ y $B / A$ es de dimensión finita sobre $k$, entonces $L \cap \widehat{B_{S}}$ localmente libre de tipo finito y $\widehat{V_{S}} / L+\widehat{B_{S}}=0$.

3. $\widehat{V_{S}} / L+\widehat{B_{S}}$ es localmente de presentación finita.

4. Si $\widehat{V_{S}} / L+\widehat{B_{S}}=0$ entoces $L \cap \widehat{B_{S}}$ es localmente libre de tipo finito.

\section{Demostración.}

1. Como $\widehat{V_{S}} / L+\widehat{A_{S}}=0$ se tiene la sucesión exacta:

$$
0 \rightarrow L \cap \widehat{A_{S}} \rightarrow L \rightarrow(\widehat{V / A})_{S} \rightarrow 0
$$

y por ser $\left(\widehat{V / A)_{S}}=\widehat{V_{S}} / \widehat{A_{S}}=(V / A)_{S}\right.$ plano se deduce que para todo $s \in S$ es $\left(L \cap \widehat{A_{S}}\right)_{s}=L_{s} \cap \widehat{A_{s}}$. Por Nakayama, al ser $L \cap \widehat{A_{S}}$ de tipo finito, existe un entorno abierto $U$ de $s$ en $S$ tal que:

$$
\left(L \cap \widehat{A_{S}}\right)_{U}=L_{U} \cap \widehat{A_{U}}
$$


2. Se tiene que $(B / A)_{S}$ es localmente libre de tipo finito, $\widehat{V_{S}} / L+\widehat{A_{S}}=0$ y que $L \cap \widehat{A_{S}}$ localmente libre de tipo finito por hipótesis. Se concluye sustituyendo en la sucesión A.0.1).

3. Sea $C=A+B(C \in \tau$ por $A, B \in \tau)$, entonces $A \subset C$ y $C / A$ de dimensión finita sobre $k$ (pues $A \sim C$ ) y aplicando el apartado 2. resulta que $L \cap \widehat{C_{S}}$ localmente libre de tipo finito y $\widehat{V_{S}} / L+\widehat{C_{S}}=0$. También tenemos que $B \subset C$ y $C / B$ de dimensión finita, aplicando $\widehat{\text { A.0.1 }}$ a $B \subset C$ se concluye que $\widehat{V_{S}} / L+\widehat{B_{S}}$ es el conúcleo de dos módulos localmente libres de tipo finito, luego localmente de presentación finita.

4. Sea $C$ como antes, si $\widehat{V_{S}} / L+\widehat{B_{S}}=0$, de $\widehat{\text { A.0.1 }}$ se deduce la sucesión:

$$
0 \rightarrow L \cap \widehat{B_{S}} \rightarrow L \cap \widehat{C_{S}} \rightarrow\left(\widehat{C / B}_{S} \rightarrow 0\right.
$$

por lo que se concluye.

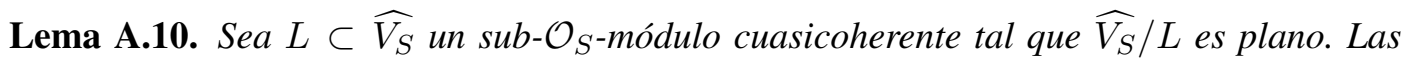
siguientes condiciones son equivalentes:

1. Para todo $s \in S$ existe un entorno abierto $U$ de $s$ en $S$ y un subespacio $A \in \tau$ tal que $L_{U} \cap \widehat{A_{U}}$ y $\widehat{V_{U}} / L_{U}+\widehat{A_{U}}$ son localmente libres de tipo finito.

2. Para todo $s \in S$ existe un entorno abierto $U$ de s en $S$ y un subespacio $A \in \tau$ tal que $L_{U} \cap \widehat{A_{U}}=0$ y $\widehat{V_{U}} / L_{U}+\widehat{A_{U}}$ localmente libre de tipo finito.

3. Para todo $s \in S$ existe un entorno abierto $U$ de s en $S$ y un subespacio $A \in \tau$ tal que $L_{U} \oplus \widehat{A_{U}} \simeq \widehat{V_{U}}$.

4. Para todo $s \in S$ existe un entorno abierto $U$ de s en $S$ y un subespacio $A \in \tau$ tal que $L_{U} \cap \widehat{A_{U}}$ localmente libre de tipo finito y $\widehat{V_{U}} / L_{U}+\widehat{A_{U}}=0$.

Además, si se verifica cualquiera de ellas, L es localmente libre y completo.

Demostración.

$1 . \Rightarrow 2$. Como la topología es separada, existe $B \in \tau$ contenido en $A$ tal que $L \cap \widehat{B_{S}}=0$. Teniendo en cuenta la sucesión (A.0.1) se deduce:

$$
0 \longrightarrow L_{U} \cap \widehat{A_{U}} \longrightarrow A_{U} / B_{U} \stackrel{\psi}{\longrightarrow} \widehat{V_{U}} / L_{U}+\widehat{B_{U}} \stackrel{\phi}{\longrightarrow} \widehat{V_{U}} / L_{U}+\widehat{A_{U}} \longrightarrow 0
$$

Veamos que $\widehat{V_{U}} / L_{U}+\widehat{B_{U}}$ es libre de tipo finito. Para ello rompemos la sucesión anterior en dos:

$$
\begin{gathered}
0 \rightarrow \operatorname{ker} \phi \rightarrow \widehat{V_{U}} / L_{U}+\widehat{B_{U}} \rightarrow \widehat{V_{U}} / L_{U}+\widehat{A_{U}} \rightarrow 0 \\
0 \rightarrow L_{U} \cap \widehat{A_{U}} \rightarrow A_{U} / B_{U} \rightarrow \operatorname{Im} \psi \rightarrow 0
\end{gathered}
$$


Se tiene que $\widehat{V_{U}} / L_{U}+\widehat{A_{U}}$ es libre finito generado, luego proyectivo, y por tanto la sucesión (A.0.2) rompe dando:

$$
\widehat{V_{U}} / L_{U}+\widehat{B_{U}} \simeq \operatorname{ker} \phi \oplus \widehat{V_{U}} / L_{U}+\widehat{A_{U}}
$$

Además, $\operatorname{ker} \phi=\operatorname{Im} \psi$, y como $\operatorname{Im} \psi$ es de presentación finita y por el lema A.8 $\operatorname{Im} \psi$ es puntualmente libre, resulta que es localmente libre de tipo finito . Así, $\widehat{V_{U}} / L_{U}+\widehat{B_{U}}$ es suma directa de dos libres de tipo finito, con lo que se concluye.

2. $\Rightarrow 3$. Por ser $L_{U} \cap \widehat{A_{U}}=0$ se tiene la sucesión:

$$
0 \rightarrow L_{U} \rightarrow(V / A)_{U} \rightarrow \widehat{V_{U}} / L_{U}+\widehat{A_{U}} \rightarrow 0
$$

Como $\widehat{V_{U}} / L_{U}+\widehat{A_{U}}$ es libre de tipo finito y $V / A$ contiene a todos los subespacios de dimensión finita (condiciones de la topología), existe un subespacio de dimensión finita que por la proyección $(V / A)_{s} \rightarrow \widehat{V}_{s} / L_{s}+\widehat{A}_{s} \rightarrow 0$ es isomorfo a $\widehat{V}_{s} / L_{s}+\widehat{A_{s}}$. Así pues, podemos elegir $B \in \tau$ tal que:

$$
(A+B / A)_{s} \simeq \widehat{V}_{s} / L_{s}+\widehat{A_{s}}
$$

y por Nakayama, también en un entorno abierto $U^{\prime}$ de $U$ conteniendo a $s$. Tomando $C=$ $A+B$ y aplicando la sucesión A.0.1 a $A \subset C$ y $U^{\prime}$, resulta que $L_{U}^{\prime} \oplus \widehat{C_{U}^{\prime}} \simeq \widehat{V_{U}^{\prime}}$.

3. $\Rightarrow 4$. Trivial, pues si $L_{U} \oplus \widehat{A_{U}} \simeq \widehat{V_{U}}$, entonces:

$$
L_{U} \cap \widehat{A_{U}}=\widehat{V_{U}} / L_{U}+\widehat{A_{U}}=0
$$

4. $\Rightarrow 1$.Trivial.

Además, si $L_{U} \oplus \widehat{A_{U}} \simeq \widehat{V_{U}}$, entonces se tiene que:

$$
L_{U} \simeq\left(\widehat{V / A}_{U}=(V / A)_{U}\right.
$$

de lo que se sigue que $L$ es localmente libre y completo.

Definición A.11. Dado un $k$-esquema $S$, se llama submódulo discreto de $\widehat{V_{S}}$ a todo haz de sub- $\mathcal{O}_{S}$-módulos cuasicoherente $L \subset \widehat{V_{S}}$ tal que:

1. $\widehat{V_{S}} / L$ es plano.

2. Para cada $s \in S\left(L_{k(s)} \subset\left(\widehat{V_{S}}\right)_{k(s)}\right)$ existe un entorno abierto $U$ de $s$ y un subespacio vectorial $A$ de $V$ con $A \in \tau$ tal que $\widehat{V_{U}} / L_{U}+\widehat{A_{U}}$ y $L_{U} \cap \widehat{A_{U}}$ son libres de tipo finito.

Observación A.12. Si bien esta definición es una generalización natural de la noción de subespacio discreto, a la hora de comprobar cuándo un submódulo es discreto sustituiremos la segunda condición por 4. del lema A.10.

Definición A.13. Dado un $k$-espacio vectorial linealmente topológico $(V, \tau)$, el functor Grassmanniana, $\operatorname{Gr}(V, \tau)$, es el functor contravariante sobre la categoría de $k$-esquemas definido por:

$$
\operatorname{Gr}(V, \tau)(S)=\left\{\begin{array}{c}
\text { Sub- } \mathcal{O}_{S} \text {-módulos discretos } \\
\text { de } \widehat{V_{S}} \text { con respecto a } \tau
\end{array}\right\}
$$

para cada $k$-esquema $S$. 
Para ver que nuestro functor Grassmanniana es representable utilizaremos, à la Grothendieck, que todo functor contravariante que es haz es representable si y sólo si lo es localmente, esto es, si admite un recubrimiento por subfunctores abiertos representables.

Definición A.14. Dado un subespacio vectorial $A \in \tau$, definimos el subfunctor $\mathbf{F}_{A}$ sobre la categoría de $k$-esquemas por:

$$
\mathbf{F}_{A}(S)=\left\{\text { Sub- } \mathcal{O}_{S} \text {-módulos } L \subset \widehat{V_{S}} \text { tales que } L \oplus \widehat{A_{S}}=\widehat{V_{S}}\right\}
$$

(esto es, $L \cap \widehat{A_{S}}=(0), L+\widehat{A_{S}}=\widehat{V_{S}}$ ).

Proposición A.15. Los subfunctores $\left\{\mathbf{F}_{A}, A \in \tau\right\}$ son representables por un k-esquema afin e íntegro.

Demostración. Es fácil comprobar que fijado un punto racional $L \in \mathbf{F}_{A}(\operatorname{Spec} k)$ se tiene un isomorfismo:

$$
\mathcal{H o m}_{k}(L, A) \simeq \mathbf{F}_{A}
$$

donde la asignación consiste en mandar cada morfismo a su gráfica en $V$. La representabilidad se sigue de los siguientes isomorfismos:

$$
\begin{aligned}
& \operatorname{Hom}\left(L_{S}, \lim _{B \in \tau}(A / A \cap B)_{S}\right)=\varliminf_{B \in \tau} \operatorname{Hom}\left(L_{S},(A / A \cap B)_{S}\right)= \\
& =\varliminf_{B \in \tau} \operatorname{Hom}(L, A / A \cap B)^{\bullet}(S)
\end{aligned}
$$

que es límite proyectivo de afines, luego afín (vease [17]).

Nótese que como $V$ es completo también lo es $A$, y por tanto $A=\lim _{B \in \tau} A / A \cap B$ siendo $A / A \cap B$ un $k$-espacio vectorial de dimensión finita.

Observación A.16. Recuérdese que $\operatorname{Hom}_{k}(L, A / A \cap B)$ es canónicamente isomorfo a:

$$
L^{*} \otimes_{k}(A / A \cap B)
$$

Tomemos su dual $L \otimes_{k}(A / A \cap B)^{*}$ y consideremos su álgebra simétrica $S^{\bullet}\left(L \otimes_{k}(A / A \cap\right.$ $\left.B)^{*}\right)$; si $\left\{e_{i}\right\}_{i \in I}$ es una base de L y $a_{1}^{B}, \ldots, a_{d_{B}}^{B}$ una base de $(A / A \cap B)^{*}\left(\right.$ donde $d_{B}$ es la dimensión de $A / A \cap B$ ) entoces:

$$
S^{\bullet}\left(L \otimes_{k}(A / A \cap B)^{*}\right)=k\left[x_{i j}^{B}\right] \quad x_{i j}^{B}=e_{i} \otimes a_{j}^{B}
$$

y tiene un espacio asociado:

$$
\operatorname{Spec} S^{\bullet}\left(L \otimes_{k}(A / A \cap B)^{*}\right)
$$

que como variedad algebraica es $L^{*} \otimes_{k}(A / A \cap B)$. 
Se tiene entonces que:

$$
\mathcal{U}_{A}=\lim _{\longleftarrow} \operatorname{Spec} k\left[x_{i j}^{B}\right]=\operatorname{Spec} \underset{\lim _{\longrightarrow}}{\longleftrightarrow}\left[x_{i j}^{B}\right]
$$

donde el límite se toma en los subespacios $B$ conmensurables con $V^{+}$.

Para calcular ese límite hay que tener en cuenta que solo se hace límite en $B, L$ no se mueve. Así que la base de $L$ está fija. Pero el sistema inductivo $\left\{(A / A \cap B)^{*}\right\}_{B \sim V^{+}}$se obtiene tomando duales en el sistema proyectivo $\{(A / A \cap B)\}_{B \sim V^{+}}$. Los morfismos del sistema proyectivo existen cada vez que $B^{\prime} \subseteq B$, y son los epimorfismos:

$$
A / A \cap B^{\prime} \rightarrow A / A \cap B \rightarrow 0 .
$$

Pasando al dual tenemos los morfismos (inyectivos) del sistema inyectivo dual:

$$
0 \rightarrow(A / A \cap B)^{*} \stackrel{\phi_{B B^{\prime}}}{\longrightarrow}\left(A / A \cap B^{\prime}\right)^{*}
$$

Por tanto, al hacer el límite inductivo $\lim _{\longrightarrow} \sim_{V^{+}} k\left[x_{i j}^{B}\right]$, tenemos que identificar $x_{i j}^{B}=e_{i} \otimes a_{j}^{B}$ con su imagen $e_{i} \otimes \phi_{B B^{\prime}}\left(a_{j}^{B}\right)$ cada vez que tengamos $B^{\prime} \subseteq B$. Si escribimos:

$$
\phi_{B B^{\prime}}\left(a_{j}^{B}\right)=\sum_{h=1}^{d_{B^{\prime}}} \lambda_{h j}^{B B^{\prime}} a_{h}^{B^{\prime}}, \quad \lambda_{h j}^{B B^{\prime}} \in k .
$$

tenemos que identificar $x_{i j}^{B}$ con $\sum_{h=1}^{d_{B^{\prime}}} \lambda_{h j}^{B B^{\prime}} x_{i h}^{B^{\prime}}$ en el límite inductivo. En resumen si escribimos el anillo de polinomios:

$$
P:=k\left[x_{i j}^{B}, i \in I, B \sim V^{+}, 1 \leq j \leq d_{B}\right]
$$

de todas las variables que nos han aparecido hasta ahora, se tiene que:

$$
\underset{B \sim V^{+}}{\lim _{B}} k\left[x_{i j}^{B}\right]=P / J
$$

siendo $J$ el ideal generado por las relaciones:

$$
x_{i j}^{B}-\sum_{h=1}^{d_{B^{\prime}}} \lambda_{h j}^{B B^{\prime}} x_{i h}^{B^{\prime}}
$$

para cualesquiera subespacios $B^{\prime} \subseteq B$ conmensurables con $V^{+}$y para todo $i \in I, j=$ $1, \ldots, d_{B}$.

Proposición A.17. $\left\{\mathbf{F}_{A}, A \in \tau\right\}$ son subfunctores abiertos de $\operatorname{Gr}(V, \tau)$.

Demostración. Tenemos que demostrar que dado $L \in \operatorname{Gr}(V, \tau)(S)$ existe, para cada $s \in S$ con $L_{s} \in \mathbf{F}_{A}(\operatorname{Spec} \kappa(s))$, un subconjunto abierto $U$ de $s$ en $S$ tal que:

$$
L_{U} \in \mathbf{F}_{A}(U)
$$


Como $L_{s} \in \mathbf{F}_{A}(\operatorname{Spec} \kappa(s))$ es:

$$
\widehat{V}_{s} / L_{s}+\widehat{A_{s}}=0
$$

luego al ser $\widehat{V_{S}} / L+\widehat{A_{S}}$ localmente de presentación finita (proposición A.9, el lema de Nakayama afirma que existe un entorno abierto $U$ de $s$ en $S$ tal que:

$$
\widehat{V_{U}} / L_{U}+\widehat{A_{U}}=0
$$

Por otra parte, aplicando de nuevo la proposición A.9. $L_{U} \cap \widehat{A_{U}}$ es de tipo finito y como $L_{s} \cap \widehat{A_{s}}=0$ (pues $L_{s} \in \mathbf{F}_{A}(s)$ ), por Nakayama existe un entorno abierto $U^{\prime} \subseteq U$ de $s$ tal que:

$$
L_{U^{\prime}} \cap \widehat{A_{U^{\prime}}}=0
$$

por lo que $L_{U^{\prime}} \in \mathbf{F}_{A}\left(U^{\prime}\right)$, como queríamos demostrar.

Proposición A.18. Los subfunctores abiertos $\left\{\mathbf{F}_{A}, A \in \tau\right\}$ recubren $\operatorname{Gr}(V, \tau)$.

Demostración. Se deduce del lemaA.10.

Basta entonces con probar si $\operatorname{Gr}(V, \tau)$ es contravariante y haz para ver que es representable.

Proposición A.19. $\operatorname{Gr}(V, \tau)$ es functor contravariante y fijado un k-esquema $S$ el functor $U \rightsquigarrow \operatorname{Gr}(V, \tau)(U)$ sobre la categoría de abiertos de $S$ es haz.

Demostración. Dado $f: U \rightarrow S$ con $U$ abierto de $S$ definimos el morfismo:

$$
\operatorname{Gr}(V, \tau)(S) \rightarrow \operatorname{Gr}(V, \tau)(U)
$$

que manda $L \rightarrow \widehat{L_{U}}$. Para ver que es functorial basta ver que si $U^{\prime} \rightarrow S$ es otro abierto de $S$

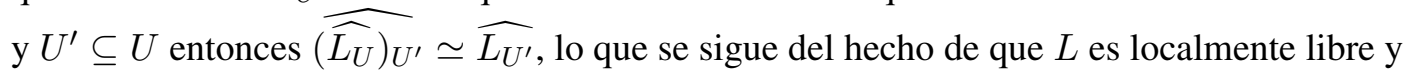
completo.

Veamos que el functor $U \rightsquigarrow \operatorname{Gr}(V, \tau)(U)$ sobre la categoría de abiertos de $S$ es haz. Sea $\left\{U_{i}\right\}_{i \in I}$ un recubrimiento por abiertos de $U$, la siguiente sucesión es exacta:

$$
0 \longrightarrow \widetilde{G r}(V)(U) \stackrel{f}{\longrightarrow} \prod_{i \in I} \widetilde{G r}(V)\left(U_{i}\right) \stackrel{f_{i}, f_{j}}{\longrightarrow} \prod_{i \neq j} \widetilde{G r}(V)\left(U_{i, j}\right)
$$

donde si $L \in \widetilde{G r}(V)(U)$ y $\left\{L_{i}\right\}_{i \in I} \in \prod_{i \in I} \widetilde{G r}(V)\left(U_{i}\right)$ se define:

$$
f(L)=L_{\mid U_{i}}, \quad f_{i}\left(\left\{L_{i}\right\}_{i \in I}\right)=L_{i_{\mid U_{i j}}}, \quad f_{j}\left(\left\{L_{i}\right\}_{i \in I}\right)=L_{j_{\mid U_{i j}}},
$$

siendo $U_{i j}=U_{i} \cap U_{j}$. En efecto: 
- Sean $L, L^{\prime} \in \widetilde{G r}(V)(U)$ tales que existen isomorfismos (de subhaces de $\widehat{V}_{\mid U_{i}}$ ) $\alpha_{i}: L_{\mid U_{i}} \stackrel{L_{\mid U_{i}}^{\prime}}{ }$ que hacen conmutativo el diagrama:

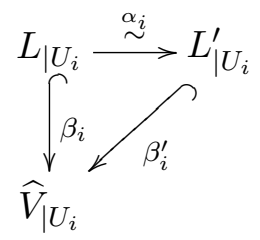

Si denotamos por $\beta_{i j}=\beta_{i_{\mid U_{i j}}}$ y $\beta_{i j}^{\prime}=\beta_{i_{\mid U_{i j}}}^{\prime}$, se sigue que:

$$
\beta_{i j}^{\prime} \circ \alpha_{i_{\mid U_{i j}}}=\beta_{i j}, \quad \beta_{i j}^{\prime} \circ \alpha_{j_{\mid U_{i j}}}=\beta_{i j},
$$

y como $\beta_{i j}^{\prime}$ son inyectivos se tiene que $\alpha_{i_{\mid U_{i j}}}=\alpha_{j_{\mid U_{i j}}}$, luego existe $\alpha: L \simeq L^{\prime}$ tal que $\alpha_{\mid U_{i}}=\alpha_{i}$.

- Sea $\left\{L_{i}\right\}_{i \in I} \in \prod_{i \in I} \widetilde{G r}(V)\left(U_{i}\right)$ tal que $\alpha_{i j}: L_{i_{\mid U_{i j}}} \simeq L_{j_{\mid U_{i j}}}$. Se tiene que $\beta_{i_{\mid U_{i j}}}=$ $\beta_{j_{\mid U_{i j}}} \circ \alpha_{i j}$, donde:

$$
\beta_{i}: L_{i} \hookrightarrow \widehat{V}_{\mid U_{i}} \quad \beta_{j}: L_{j} \hookrightarrow \widehat{V}_{\mid U_{i}}
$$

Del mismo modo $\beta_{i_{\mid U_{i j}}}=\beta_{k_{\mid U_{i j}}} \circ \alpha_{i k} \mathrm{y} \beta_{j_{\mid U_{i j}}}=\beta_{k_{\mid U_{i j}}} \circ \alpha_{j k}$ luego:

$$
\beta_{i_{\mid U_{i j k}}}=\beta_{k_{\mid U_{i j k}}} \circ \alpha_{j k_{\mid U_{i j k}}} \circ \alpha_{i j_{\mid U_{i j k}}}
$$

Como $\beta_{k}$ es inyectivo se tiene que $\alpha_{i j}$ verifica la condición de cociclo $\alpha_{i k}=\alpha_{j k} \circ \alpha_{i j}$ en $U_{i j k}$, por lo tanto existe un subhaz $L$ de $\widehat{V}_{\mid U}$ tal que $L_{\mid U_{i}} \simeq L_{i}$. Además $L \in$ $\widetilde{G r}(V)(U)$ ya que $L_{i} \in \widetilde{G r}(V)\left(U_{i}\right)$ para todo $i \in I$.

Queda demostrado el siguiente:

Teorema A.20. El functor $\operatorname{Gr}(V, \tau)$ es representable por un k-esquema que denotaremos $\operatorname{Gr}(V)$.

Calculamos ahora su espacio tangente (véase [32, Prop.4.1]).

Proposición A.21. Sea $L \in \operatorname{Gr}(V)$ un punto racional. Existe un isomorfismo canónico:

$$
\mathrm{T}_{L} \operatorname{Gr}(V) \simeq \operatorname{Hom}_{k}(L, V / L)
$$

Demostración. El espacio tangente es un noción local, por tanto para calcular $\mathrm{T}_{L} \operatorname{Gr}(V)$ basta calcular $\mathrm{T}_{L} F_{A}$ (recordemos que el functor Grassmanniana estaba recubrierto por los subfuntores abiertos representables $\mathbf{F}_{A}(S)=\left\{L \subset \widehat{V_{S}}\right.$ tales que $\left.L \oplus \widehat{A_{S}} \simeq \widehat{V_{S}}\right\}$ con $A \sim$ $\left.V_{+}\right)$. Es bien sabido que $\mathbf{F}_{A}$ es isomorfo al espacio afín $\operatorname{Hom}(L, A)$ y que la inclusión:

$$
\operatorname{Hom}(L, A) \simeq \mathbf{F}_{A} \hookrightarrow \operatorname{Gr}(V)
$$


manda $f: L \rightarrow A$ a su gráfica $\Gamma_{f}=\{l+f(l)\}$. Calculemos el espacio tangente a $\operatorname{Hom}(L, A)$ en la aplicacion 0: $L \rightarrow A$. Por definición:

$$
T_{0} \operatorname{Hom}(L, A):=\operatorname{Hom}(L \oplus \epsilon L, A \oplus \epsilon A) \times \operatorname{Hom}(L, A)\{0\}
$$

con $\epsilon^{2}=0$. Sus elementos son aplicaciones de $k[\epsilon]$-módulos:

$$
\bar{g}: L \oplus \epsilon L \rightarrow A \oplus \epsilon A
$$

haciendo conmutativo el siguiente diagrama:

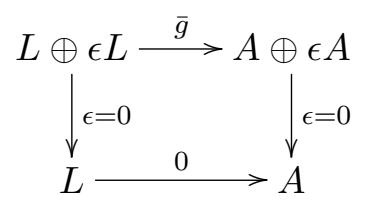

Y por tanto pueden escribirse como matrices $\bar{g}$ de la forma:

$$
\left(\begin{array}{ll}
0 & g \\
f & h
\end{array}\right)
$$

de modo que si $l, m \in L$, es:

$$
\begin{aligned}
\bar{g}(l+\epsilon m) & =\left(\begin{array}{ll}
0 & g \\
f & h
\end{array}\right)\left(\begin{array}{c}
l \\
m
\end{array}\right)\left(\begin{array}{c}
g(m) \\
f(l)+h(m)
\end{array}\right)= \\
& =g(m)+\epsilon(f(l)+h(m))
\end{aligned}
$$

Estas matrices han de ser lineales en $\epsilon$, es decir, tienen que verificar:

$$
\bar{g}(\epsilon(l+\epsilon m))=\epsilon \bar{g}(l+\epsilon m) .
$$

Como:

$$
\bar{g}(\epsilon(l+\epsilon m))=\bar{g}(\epsilon l)=g(l)+\epsilon h(l) \quad \text { y } \quad \epsilon \bar{g}(l+\epsilon m)=\epsilon g(m),
$$

tiene que ser:

$$
g(l)=0 \quad \text { y } h(l)=g(m)
$$

y por tanto $h=g=0$. Es decir, $g$ es de la forma:

$$
\bar{g}=\left(\begin{array}{cc}
0 & 0 \\
f & 0
\end{array}\right) .
$$

Se tiene entonces un isomorfismo de $k$-espacios vectoriales:

$$
\begin{aligned}
\operatorname{Hom}(L, A) & \simeq \mathrm{T}_{0} \operatorname{Hom}(L, A) \\
f & \mapsto\left(\begin{array}{ll}
0 & 0 \\
f & 0
\end{array}\right)
\end{aligned}
$$


y por tanto:

$$
\operatorname{Hom}(L, A) \simeq \mathrm{T}_{0} \operatorname{Hom}(L, A) \simeq \mathrm{T}_{0} \operatorname{Gr}(V) \simeq \operatorname{Gr}(V)\left(k[\epsilon] / \epsilon^{2}\right) \times_{\operatorname{Gr}(V)(k)}\{L\}
$$

mandando $f \in \operatorname{Hom}(L, A)$ al punto de $\operatorname{Gr}(V)$ con valores en $k[\epsilon] / \epsilon^{2}$ dado por la gráfica de $\bar{f}=\left(\begin{array}{cc}0 & 0 \\ f & 0\end{array}\right)$, es decir, a:

$$
\Gamma_{\bar{f}}=\{l+\epsilon m+\epsilon f(l) \mid l, m \in L\}
$$

que como $k[\epsilon]$-módulo equivale a:

$$
\Gamma_{\bar{f}}=\{l+\epsilon f(l) \mid l \in L\}
$$

(nótese que $\epsilon(l+\epsilon m+\epsilon f(l))=\epsilon l$ ). Componiendo la inversa de esta aplicación con el isomorfismo:

$$
\begin{aligned}
\operatorname{Hom}(L, A) & \simeq \operatorname{Hom}(L, V / L) \\
f & \mapsto L \stackrel{\text { Id }+f}{\rightarrow} L \oplus A \simeq V \rightarrow V / L
\end{aligned}
$$

obtenemos el isomorfismo del enunciado (nótese que no depende de la elección de $A$ ). 


\section{Apéndice B}

\section{De la Grassmanniana al moduli de curvas.}

Recordamos en este apédice cómo recuperar una curva, un divisor de Cartier liso y una trivialización formal de la curva en el divisor a partir de un dato en la Grassmanniana infinita de $k((z))$ (véase [35], [28] y [33]).

Sea $S$ un $k$-esquema y $A \in \operatorname{Gr}(k((z)))(S)$. Como $\operatorname{Gr}(k((z)))$ es haz podemos suponer sin pérdida de generalidad que $S=\operatorname{Spec} \mathcal{O}$ es el espectro de un anillo local. Supongamos que $A$ es una sub- $\mathcal{O}$-álgebra no trivial de $\mathcal{O}((z))$.

Definimos una filtración en $A$ poniendo:

$$
\begin{gathered}
A^{(i)}=A \cap z^{-i} \mathcal{O}[[z]] \quad \text { para cada entero } i \geq 0 \\
\cdots \subset A^{(i-1)} \subset A^{(i)} \subset A^{(i+1)} \subset \cdots
\end{gathered}
$$

y recordemos (apéndice A) que $k((z))$ tiene un topología $\tau$ formada por el conjunto de subespacios conmensurables con $k[[z]]$ que contienen a $z^{m} k[[z]]$ para algún $m \in \mathbb{Z}$ (donde se dice que dos subespacios, $B$ y $C$, de $k((z))$ son conmensurables si $B+C / B \cap C$ es un $k$-espacio vectorial de dimensión finita).

Lema B.1. Para $i \gg 0$, existe un elemento en $A^{(i)}$ de la forma:

$$
p_{A, i}=z^{-i}+\text { términos de grado mayor en } z \text {. }
$$

Demostración. Como $S$ es local, se tiene $\mathcal{O}((z))=A \oplus \widehat{B}_{S}$ para $B$ en la topología. Por tanto $\widehat{B}_{S}$ tiene una base sobre $\mathcal{O}$ de la forma:

$$
\left(z^{m_{0}}, z^{m_{1}}, \ldots, z^{m_{j}}, \ldots, z^{m_{h}}, \ldots,\right)
$$

donde $m_{0}<m_{1}<\cdots<m_{j}$ y $m_{j+h}=m_{j}+h$ para $h \geq 0$ (pues $B$ ha de contener a $z^{n} k[[z]]$ para algún $\left.n \in \mathbb{N}\right)$. Si $-i<m_{0}$, al escribir $z^{-i} \in \mathcal{O}((z))=A \oplus \widehat{B}_{S}$ como:

$$
z^{-i}=p_{A, i}+q_{i}, \quad p_{A, i} \in A, \quad q_{i} \in \widehat{B}_{S},
$$

el elemento $q_{i}$ no puede tener coeficiente en $z^{-i}$, luego $p_{A, i}$ es de la forma pedida. 
Consideremos la inclusión natural:

$$
A^{(i)} / A^{(i-1)} \hookrightarrow z^{-i} \mathcal{O}[[z]] / z^{-(i-1)} \mathcal{O}[[z]] \simeq z^{-i} \mathcal{O}
$$

Lema B.2. Para $i \gg 0$ como en el Lema anterior, la inclusión anterior es una igualdad:

$$
A^{(i)} / A^{(i-1)} \simeq z^{-i} \mathcal{O}[[z]] / z^{-(i-1)} \mathcal{O}[[z]] \simeq z^{-i} \mathcal{O}
$$

que transforma la clase $\bar{p}_{i}$ de $p_{i}$ en $A^{(i)} / A^{(i-1)}$ en $z^{-i}$.

Demostración. Como $z^{-i}=p_{i}+q_{i}$ y $q_{i} \in z^{-i+1} \mathcal{O}[[z]]$, la clase $\bar{p}_{i}$ de $p_{i}$ en $z^{-i} \mathcal{O}[[z]] / z^{-(i-1)} \mathcal{O}[[z]]$ es igual a la clase de $z^{-i}$.

Lema B.3. Fijado $m$, para $i-m \gg 0$ como en el Lema anterior, se tiene:

$$
A^{(i)} / A^{(i-m)} \simeq z^{-i} \mathcal{O}[[z]] / z^{-(i-m)} \mathcal{O}[[z]]=z^{-i} \mathcal{O} \oplus \cdots \oplus z^{-(i-m+1)} \mathcal{O} .
$$

Demostración. Se procede por recurrencia sobre $m$, siendo el caso $m=1$ el Lema anterior. Se tiene un diagrama:

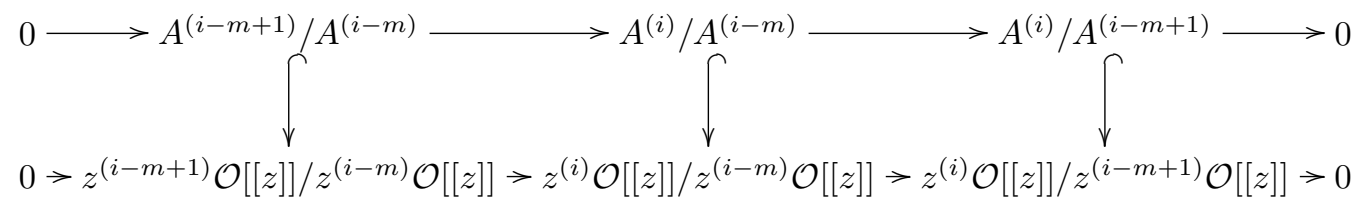

La primera columna es isomorfismo por el Lema anterior, y la última por recurrencia, luego también es isomorfismo la columna central.

Sea $\mathcal{A}:=\oplus_{i \geq 0} x_{1}^{i} A^{(i)}$ el álgebra graduada a esta filtración (donde $x_{1}$ es una variable independiente que introducimos para graduar con el fin de distinguir un elemento de $A^{(i)}$ de él mismo pensado dentro de $\left.A^{(i+1)}\right)$. Como $A$ es una sub- $k$-álgebra no trivial de $k((z))$ se sigue que $X=\operatorname{Proj} \mathcal{A}$ es una curva sobre $S$. Veamos que tenemos definida de modo natural una sección $\sigma: S \rightarrow X$ cuyo divisor de Cartier asociado, $D=\sigma(S)$, es el subesquema de $X$ definido por el ideal $x_{1} \mathcal{A}$ y que recuperamos la trivialización formal de $X$ a lo largo de $D$ dando $\mathcal{A} \rightarrow \mathcal{A} / x_{1}^{m}$.

Observación B.4. Si consideramos el anillo graduado $\mathcal{A}^{n}:=\oplus_{i \geq 0} x_{1}^{i} A^{(i n)}$, la multiplicación por $x_{1}^{n}$ es una inclusión $\mathcal{A}^{n} \subseteq \mathcal{A}$ cuya imagen es el anillo graduado truncado $\mathcal{A}^{[n]}$, luego tienen el mismo Proj, es decir, dan la misma familia de curvas $\operatorname{Proj} \mathcal{A} \simeq \operatorname{Proj} \mathcal{A}^{n}$.

Gracias a la variable $x_{1}$ es fácil ver que la localización homogénea de $\mathcal{A}$ en $x_{1}$ es precisamente $A$. En efecto:

$$
\mathcal{A}_{x_{1}}^{+}=\left\{\frac{x_{1}^{i} a_{i}}{x_{1}^{i}}, a_{i} \in A^{(i)} \text { para todo } i\right\}=\cup_{i \geq 0} A^{(i)}=A
$$


Así, el abierto básico $V_{x_{1}}^{+}$definido por $x_{1}$ es isomorfo a Spec $A$ y por tanto, el complementario de Spec $A$ en $X$ se corresponde con los ceros homogéneos de $x_{1}$. Veamos que de modo natural tenemos definida una sección de $\pi: X \rightarrow S$. Consideremos:

$$
0 \rightarrow \mathcal{A}[-1] \stackrel{\cdot x_{1}}{\longrightarrow} \mathcal{A} \rightarrow \oplus_{i \geq 0} A^{(i)} / A^{(i-1)} \rightarrow 0
$$

donde el paso de $\mathcal{A}[-1]$ a $\mathcal{A}$ consiste en multiplicar por $x_{1}$. Aplicando los lemas B.1 y B.2 resulta que se tiene $A^{(i)} / A^{(i-1)} \simeq z^{-i} \mathcal{O}$ para $i \gg 0$, luego considerando una nueva variable independiente $x_{0}$ podemos definir, para $n \gg 0$, un morfismo homogéneo:

$$
\mathcal{O}\left[x_{0}\right]^{[n]} \rightarrow\left(\oplus_{i \geq 0} x_{1}^{i} A^{(i)} / A^{(i-1)}\right)^{[n]}
$$

(donde ${ }^{[n]}$ denota tomar la truncación de grado $n$ ) mandando $x_{0}^{i}$ a $x_{1}^{i} z^{-i}$ para $i \gg 0$ y a cero en otro caso, que es de hecho un isomorfismo para grados altos (de nuevo $x_{1}$ sirve para graduar). Tomando Proj resulta:

$$
\begin{gathered}
\operatorname{Proj} \oplus_{i \geq 0} x_{1}^{i} A^{(i)} / A^{(i-1)} \simeq \operatorname{Proj} \oplus_{i \geq 0} x_{1}^{i n} A^{(i n)} / A^{(i-1) n} \simeq \\
\simeq \operatorname{Proj} \mathcal{O}\left[x_{0}\right]^{[n]} \simeq \operatorname{Proj} \mathcal{O}\left[x_{0}\right] \simeq \operatorname{Spec} \mathcal{O}=S
\end{gathered}
$$

y teniendo en cuenta la sucesión exacta $(\overline{B .0 .2})$ construimos la sección:

$$
\sigma: S \hookrightarrow \operatorname{Proj} \mathcal{A}=X
$$

cuyo divisor de Cartier asociado, $D=\sigma(S)$, es el subesquema cerrado de $X$ definido por el ideal $x_{1} \mathcal{A}$, y da para cada punto geométrico $s=\operatorname{Spec} \kappa(s) \in S$ un verdadero punto $p=\operatorname{Proj} \kappa(s)\left[x_{0}\right]$ en la fibra $X_{s}=X \times_{S} \operatorname{Spec} \kappa(s)$ de $X$ sobre $s$ (donde denotamos por $\kappa(s)$ al cuerpo residual del punto $s \in S)$.

Para probar que $p$ es un punto liso en $X$, o más en general que $X$ es liso a lo largo de $D$, bastará ver que la completación formal del haz $\mathcal{O}_{X}$ a lo largo de $D$ es isomorfa a un anillo de series formales. En efecto, $D$ es el subesquema de $X$ dado por el ideal $\left(x_{1}\right)$ y $x_{0} \notin\left(x_{1}\right)$, luego $D \in X-\left(x_{0}\right)_{0}^{+}=\operatorname{Spec} \mathcal{A}_{x_{0}}^{+}$y el ideal que define en $\mathcal{A}_{x_{0}}^{+}$es $\left(x_{1}\right)_{x_{0}}^{+}=\left(\frac{x_{1}}{x_{0}}\right)$. Se tiene así:

$$
\widehat{\mathcal{O}}_{X, D}=\underset{m}{\lim }\left(\left(\mathcal{A} / x_{1}^{m}\right)_{x_{0}}^{+}\right)\left(\frac{x_{1}}{x_{0}}\right)
$$

Consideremos $\mathcal{A} /\left(x_{1}^{m}\right)$; la componente homogénea de grado $i$ de este anillo graduado viene dada por $x_{1}^{i} \cdot A^{(i)} / A^{(i-m)}$, donde de nuevo se pone la variable $x_{1}$ para graduar. Por el lema B.3. $A^{(i)} / A^{(i-m)}$ es isomorfo para todo $i>m \gg 0$ a:

$$
z^{-i} \mathcal{O}[[z]] / z^{-(i-m)} \mathcal{O}[[z]]=z^{-i} \mathcal{O} \oplus \cdots \oplus z^{-(i-m+1)} \mathcal{O}
$$

Por tanto:

$$
x_{1}^{i} \cdot A^{(i)} / A^{(i-m)} \simeq\left\langle x_{0}^{i}, x_{0}^{i-1} x_{1}, \ldots, x_{0}^{i-m+1} x_{1}^{m-1}\right\rangle
$$

(donde $x_{0}=z^{-1} x_{1}$ ), que es isomorfo a su vez a la componente de grado $i$ de $\mathcal{O}\left[x_{0}, x_{1}\right] / x_{1}^{m}$. Luego:

$$
\mathcal{A} /\left(x_{1}^{m}\right) \simeq \mathcal{O}\left[x_{0}, x_{1}\right] /\left(x_{1}^{m}\right)
$$


para $m \gg 0$ y entonces:

$$
\left(\mathcal{A} /\left(x_{1}^{m}\right)\right)_{x_{0}}^{+}=\mathcal{O}\left[\frac{x_{1}}{x_{0}}\right] /\left(\frac{x_{1}}{x_{0}}\right)^{m}
$$

Se concluye que:

$$
\widehat{\mathcal{O}}_{X, D}={\underset{\lim }{m}}_{\operatorname{lo}}\left(\mathcal{O}\left[\frac{x_{1}}{x_{0}}\right] /\left(\frac{x_{1}}{x_{0}}\right)^{m}\right)_{\left(\frac{x_{1}}{x_{0}}\right)}=\mathcal{O}\left[\left[\frac{x_{1}}{x_{0}}\right]\right]_{\left(\frac{x_{1}}{x_{0}}\right)}=\mathcal{O}\left[\left[\frac{x_{1}}{x_{0}}\right]\right] \simeq \mathcal{O}[[z]]
$$

Hemos visto además que se puede elegir $z$ como parámetro formal. 


\section{Apéndice C}

\section{Operadores diferenciales.}

Exponemos aquí un breve repaso de la teoría de operadores diferenciales desarrollada en [17, 16.8.8].

Definición C.1. Sea $f: X \rightarrow S$ un morfismo de esquemas y denotemos:

$$
\Delta_{f}: X \rightarrow X \times_{S} X
$$

la inmersión diagonal. Denotamos $\mathcal{P}_{X / S}^{n}$ al haz de partes principales:

$$
\mathcal{O}_{X \times{ }_{S} X} / \Delta^{n+1}
$$

de orden $n$ del $S$-esquema $X$.

Denotamos $p_{1}, p_{2}$ las dos proyecciones canónicas en $X$ del producto $X \times_{S} X$. Como $\Delta_{f}$ es una $X$-sección de $X \times_{S} X$ para ambas proyecciones, se sigue que $p_{1}$ y $p_{2}$ definen, para todo $n$, dos estructuras de $\mathcal{O}_{X}$-álgebra en $\mathcal{P}_{X / S}^{n}$. Mientras no se diga lo contrario, $\mathcal{P}_{X / S}^{n}$ será considerado como $\mathcal{O}_{X}$-álgebra vía $p_{1}$ y denotaremos $d^{n}: \mathcal{O}_{X} \rightarrow \mathcal{P}_{X / S}^{n}$ al morfismo de anillos que induce $p_{2}$. Para todo abierto $U$ de $X$ y todo $a \in \Gamma\left(U, \mathcal{O}_{X}\right)$, llamamos $d^{n} a$ a la parte principal de orden $n$ de $a$. Escribiremos $d a=d^{1} a-a$ y diremos que es la diferencial de $a$ (elemento de $\Gamma\left(U, \Omega_{X / S}^{1}\right)$ ).

Denotemos $X_{\Delta}^{(n)}$ el $n$-ésimo entorno infinitesimal de $X$ por el morfismo diagonal $\Delta: X \rightarrow$ $X \times_{S} X$ y escribamos:

$$
h_{n}: X_{\Delta}^{(n)} \rightarrow X \times{ }_{S} X
$$

Consideremos el siguiente diagrama:

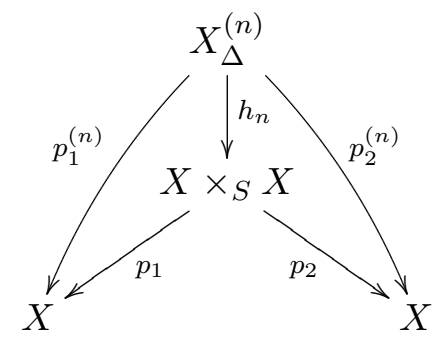


de manera que $p_{1}^{(n)}$ corresponde al morfismo de haces de anillos $\mathcal{O}_{X} \rightarrow \mathcal{P}_{X / S}^{n}$ que hemos elegido para definir la estructura de $\mathcal{O}_{X}$-álgebra de $\mathcal{P}_{X / S}^{n}$, y $p_{2}^{(n)}$ al morfismo $d^{n}: \mathcal{O}_{X} \rightarrow$ $\mathcal{P}_{X / S}^{n}$. Como $X_{\Delta}^{(n)}$ y $X$ tiene el mismo espacio subyacente, podemos escribir:

$$
\mathcal{P}_{X / S}^{n}=p_{1, *}^{(n)} p_{2}^{(n), *} \mathcal{O}_{X}
$$

o en general, para un haz de $\mathcal{O}_{X}$-módulos $E$ :

$$
\mathcal{P}_{X / S}^{n}(E)=p_{1, *}^{(n)} p_{2}^{(n), *} E
$$

de manera que $\mathcal{P}_{X / S}^{n}=\mathcal{P}_{X / S}^{n}\left(\mathcal{O}_{X}\right)$. Existe entonces un morfismo de haces:

$$
\begin{aligned}
d_{E}^{n}: E & \rightarrow \mathcal{P}_{X / S}^{n}(E) \\
e & \mapsto 1 \otimes e
\end{aligned}
$$

para $e \in \Gamma(U, E)$, luego estamos tomando:

$$
d a=d^{1} a-a=1 \otimes a-a \otimes 1 .
$$

$\mathcal{P}_{X / S}^{n}(E)$ tiene una estructura de $\mathcal{O}_{X}$-bimódulo, tomamos la estructura por la izquierda como la definida por el morfismo $\mathcal{O}_{X} \rightarrow \mathcal{P}_{X / S}^{n}$ y por la derecha la dada por $d^{n}$. Si $a \in \Gamma\left(U, \mathcal{O}_{X}\right)$, $p \in \Gamma\left(U, \mathcal{P}_{X / S}^{n}\right)$ y $e \in \Gamma(U, E)$ se tienen las siguientes relaciones:

$$
\begin{gathered}
a(p \otimes e)=(a p) \otimes e, \quad(p \otimes e) a=\left(p d^{n} a\right) \otimes e=p \otimes(a e)=\left(d^{n} a\right)(p \otimes e) \\
d_{E}^{n}(a e)=(1 \otimes e) a=\left(d_{E}^{n} e\right) a, \quad d_{E}^{n}(a e)=d^{n} a(1 \otimes e)=d^{n} a d_{E}^{n} e
\end{gathered}
$$

Definición C.2. Sea $f: X \rightarrow S$ un morfismo de esquemas localmente de presentación finita, $E$ y $F$ dos haces de $\mathcal{O}_{X}$-módulos cuasi-coherentes con $E$ de presentación finita y $n$ un entero $\geq 0$. Diremos que un morfismo de haces de grupos aditivos $P: E \rightarrow F$ es un operador diferencial de orden $\leq n$ (relativo a $S$ ) si existe un morfismo de $\mathcal{O}_{X}$-módulos $u: \mathcal{P}_{X / S}^{n}(E) \rightarrow F$ (donde dotamos a $\mathcal{P}_{X / S}^{n}(E)$ de su estructura de $\mathcal{O}_{X}$-módulo por la izquierda) tal que $P=u \circ d_{E}^{n}$. $F$.

Denotaremos $\mathcal{D i f f}_{X / S}^{n}(E, F)$ el haz de operadores diferenciales de orden $\leq n$ de $E$ en

Se tiene que:

$$
\mathcal{H}_{\mathrm{om}_{\mathcal{O}_{X}}}\left(\mathcal{P}_{X / S}^{n}(E), F\right) \stackrel{\sim}{\mathcal{D}} \operatorname{iff}_{X / S}^{n}(E, F) .
$$

Proposición C.3. Las siguientes condiciones son equivalentes:

1. $P \in \mathcal{D i f f}_{X / S}^{n}(E, F)$. 
2. Sea $U$ un abierto de $X$. Para todo $a \in \Gamma\left(U, \mathcal{O}_{X}\right)$ el morfismo:

$$
D(a): E_{\mid U} \rightarrow F_{\mid U}
$$

que para cada sección e de $E$ en un abierto $V \subset U$ está definido por:

$$
D(a)(e)=P(a e)-a P(e),
$$

define un operador diferencial de orden $\leq n-1$.

Estudiemos con un poco más de detalle el caso $n=1$, pues es el que nos interesa para este trabajo. Tomaremos $E$ y $F$ localmente libres.

Se tiene la siguiente sucesión sucesión exacta en $X \times X$ :

$$
0 \rightarrow \Delta / \Delta^{2} \rightarrow \mathcal{O}_{X \times{ }_{S} X} / \Delta^{2} \rightarrow \mathcal{O}_{X \times{ }_{S} X} / \Delta \rightarrow 0
$$

que se traduce en la siguiente sucesión exacta en $X \simeq \Delta$ :

$$
0 \rightarrow \Omega_{X / S} \rightarrow \mathcal{P}_{X / S}^{1} \rightarrow \mathcal{O}_{X} \rightarrow 0 .
$$

Tensorializando esta última sucesión exacta por $E$ obtenemos:

$$
0 \longrightarrow \Omega_{X / S} \otimes_{\mathcal{O}_{X}} E \stackrel{\alpha}{\longrightarrow} \mathcal{P}_{X / S}^{1}(E) \longrightarrow E \longrightarrow 0
$$

y tomando $\mathcal{H}_{\mathrm{om}} \mathcal{O}_{X}(-, F)$ resulta:

$$
0 \rightarrow \mathcal{H} \mathrm{m}_{\mathcal{O}_{X}}(E, F) \rightarrow \mathcal{H} \mathrm{om}_{\mathcal{O}_{X}}\left(\mathcal{P}_{X / S}^{1}(E), F\right) \rightarrow \mathcal{H} \mathrm{om}_{\mathcal{O}_{X}}\left(\Omega_{X / S} \otimes E, F\right) \rightarrow 0
$$

donde el último morfismo manda $u \in \mathcal{H}^{\circ} \operatorname{O}_{\mathcal{O}_{X}}\left(\mathcal{P}_{X / S}^{1}(E), F\right)$ a $u \circ \alpha=\psi$.

Observemos los diagramas:
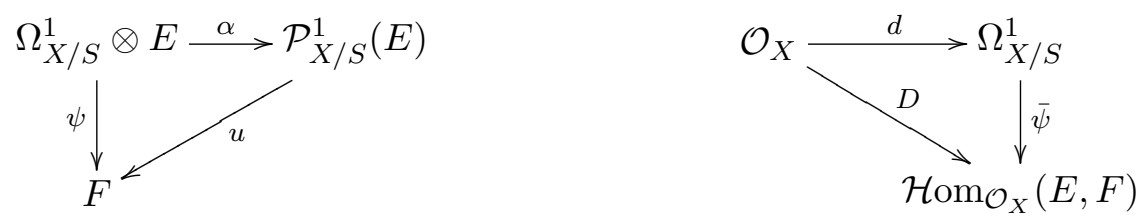

donde $d$ es la diferencial ordinaria de $X$ y:

$$
\bar{\psi}(w)(e):=\psi(w \otimes e) \quad \bar{\psi}(d a)=D(a) .
$$

Se tienen los siguientes isomorfismos:

$\mathcal{H}_{\mathrm{O}_{\mathcal{O}_{X}}}\left(\Omega_{X / S}^{1} \otimes E, F\right) \simeq \mathcal{H}_{\mathrm{om}_{\mathcal{O}_{X}}}\left(\Omega_{X / S}^{1}, \mathcal{H}_{\mathrm{om}_{\mathcal{O}_{X}}}(E, F)\right) \simeq \operatorname{Der}_{\mathcal{O}_{S}}\left(\mathcal{O}_{X}\right) \otimes \mathcal{H}_{\mathrm{om}_{\mathcal{O}_{X}}}(E, F)$ definidos por:

$$
\psi \mapsto \bar{\psi} \mapsto D
$$


Se tiene entonces una sucesión:

$0 \longrightarrow \mathcal{H}_{\mathrm{om}_{\mathcal{O}_{X}}}(E, F) \longrightarrow \mathcal{D i f f}_{X / S}^{1}(E, F) \stackrel{\sigma}{\longrightarrow} \operatorname{Der}_{\mathcal{O}_{S}}\left(\mathcal{O}_{X}\right) \otimes \mathcal{H} \mathrm{om}_{\mathcal{O}_{X}}(E, F) \longrightarrow 0$

donde el morfismo $\sigma$ manda $u$ a $D$. El morfismo $\sigma$ se llama morfismo símbolo.

Observemos además que:

$$
\begin{aligned}
D(a)(e) & =(\bar{\psi}(d a))(e)=\psi(d a \otimes e)=(u \circ \alpha)(d a \otimes e)= \\
& =u((1 \otimes a-a \otimes 1) \otimes e)=u(1 \otimes a e-a \otimes e) .
\end{aligned}
$$

Veamos, de acuerdo con la proposición anterior, que todo operador:

$$
P \in \mathcal{D i f f}_{X / S}^{1}(E, F) \simeq \mathcal{H}_{\mathrm{om}_{\mathcal{O}_{X}}}\left(\mathcal{P}_{X / S}^{1}(E), F\right)
$$

está caracterizado por la fórmula:

$$
P(a e)=a P(e)+D(a)(e) .
$$

En efecto, como $P=d_{E}^{1} \circ u\left(\operatorname{con} u \in \mathcal{H}_{\mathrm{om}_{\mathcal{O}_{X}}}\left(\mathcal{P}_{X / S}^{1}(E), F\right)\right)$ se tiene:

$$
\begin{aligned}
P(a e) & =u\left(d_{E}^{1}(a e)\right)=u(1 \otimes a e)=u(1 \otimes a e-a \otimes e+a \otimes e)= \\
& =u(1 \otimes a e-a \otimes e)+u(a \otimes e)=D(a)(e)+a u(1 \otimes e)= \\
& =D(a)(e)+a P(e) .
\end{aligned}
$$

Observación C.4. En particular, podemos tomar $E=F$ y considerar los operadores diferenciales con símbolo escalar:

$$
\mathcal{D}_{X / S}^{1}(E):=\left\{P \in \mathcal{D i f f f}_{X / S}^{1}(E, E) \mid \sigma(P) \in \operatorname{Der}_{\mathcal{O}_{S}}\left(\mathcal{O}_{X}\right) \otimes_{\mathcal{O}_{X}} \mathcal{O}_{X}\right\}
$$

donde $\mathcal{O}_{X}$ se mete en $\mathcal{E}$ nd $_{X} E$ por la diagonal. Se tiene entonces la siguiente sucesión exacta:

$$
0 \longrightarrow \mathcal{E n d}_{X} E \longrightarrow \mathcal{D}_{X / S}^{1}(E) \stackrel{\sigma}{\longrightarrow} \operatorname{Der}_{\mathcal{O}_{S}}\left(\mathcal{O}_{X}\right) \longrightarrow 0
$$

y por lo tanto $D(a)$ es simplemente una homotecia:

$$
D(a)(e)=D(a) \cdot e .
$$

En consecuencia, todo operador diferencial $P \in \mathcal{D}_{X / S}^{1}(E)$ está caracterizado por la fórmula:

$$
P(a e)=a P(e)+D(a) e, \quad D=\sigma(P) \in \operatorname{Der}_{\mathcal{O}_{S}}\left(\mathcal{O}_{X}\right) .
$$

Observación C.5. Tomando $E=\mathcal{O}_{X}$ se tiene:

$$
0 \rightarrow F \rightarrow \operatorname{Diff}_{X / S}^{1}\left(\mathcal{O}_{X}, F\right) \rightarrow \operatorname{Der}_{S}\left(\mathcal{O}_{X}\right) \otimes F \rightarrow 0
$$

y el morfismo $P \mapsto P(1)$ es un retracto de $F \rightarrow \mathcal{D i f f}_{X / S}^{1}\left(\mathcal{O}_{X}, F\right)$, es decir, todo $P \in$ $\mathcal{D i f f}_{X / S}^{1}\left(\mathcal{O}_{X}, F\right)$ se escribe de manera única $f+D$ con $f: \mathcal{O}_{X} \rightarrow F \mathcal{O}_{X}$-lineal y $D$ una derivación sobre $S$. 
Observación C.6. Tomando $S=k, E=\mathcal{O}_{X}$ y $G=\mathcal{O}_{X}(\bar{m}) / \mathcal{O}_{X}(-m)$ tenemos:

$$
\underset{\vec{m}}{\lim _{m}} \lim _{m} \mathcal{D i f f}_{X / k}^{1}\left(\mathcal{O}_{X}, \mathcal{O}_{X}(\bar{m}) / \mathcal{O}_{X}(-m)\right) \simeq \mathcal{D}_{k((z)) / k}^{1}(k((z)), k((z))) .
$$

Análogamente:

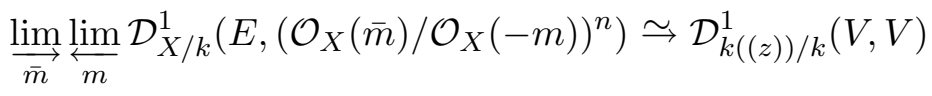




\section{Apéndice D}

\section{Quick overview of the work.}

Let us summarize in this appendix the contents of the work 1

\section{D.0. Introduction.}

During the years $77-81$, Krichever realized about the close relationship between the theory of KP systems and the theta functions of Jacobians of curves ([22]) and Mumford ([30]) wrote down a complete dictionary among different geometric data (pointed curves, line bundles, parabolic bundles, ...) and commutative subrings of some big non-commutative ring of operators. Sato also found an equivalence between the solutions of the KP system and points of an infinite Grassmannian ([40]). All these results have contributed to develop a new algebro-geometric point of view of the study of the commutativity of certain differential operators given by Burchnall and Chaudy ([11]) in the 20's.

Bearing in mind all these ideas and its relationship with the Schottky problem, in [27] (1984) Mulase characterized Jacobians as finite dimensional orbits of certain flows in the Sato Grassmannian and Shiota gave in [44] a necessary and sufficient condition for a theta function of a principally polarized abelian variety to be the theta function of a Jacobian. In 1985, Segal and Wilson formalized in [42] the Krichever morphism (a map from geometric data to the Sato Grassmannian) and made special emphasis on how can loop groups fit into these infinite systems.

In the year 1991, Mulase contributed again to develop these ideas by giving a categorical equivalence between vector bundles and curves and the Sato Grassmannian ([28]). In [24], Li and Mulase presented the KP system as certain commutative flows on the Sato Grassmannian and proved that every finite dimensional orbit of these flows is canonically isomorphic to the Jacobian of a curve (and conversely). Moreover they have related Prym varieties with the BKP system.

\footnotetext{
${ }^{1}$ For the sake of clarity, we have chosen the same numbering as the chapters of the work
} 
In the same spirit, Adams and Bergvelt studied the case of finite coverings of curves with line bundles upstairs $([1], 1992)$, showing that Heisenberg algebras define flows on the Sato Grassmannian and give raise to classes of solutions to multi-component KP-hierarchies. They provide with full detail the correspondence among geometric data, Heisenberg algebras and points in the Sato Grassamnnian.

In [3] (1996), Álvarez Vázquez, Muñoz Porras and Plaza Martín have endowed the Sato Grassmannian with a scheme structure. This have allowed them to give an algebraic theory for the KP-hierarchy over an arbitrary field and also for Tau and Baker-Akhiezer functions (these functions play an important role if one wants to compute explicit equations). Following the ideas of Segal ([41]), Muñoz Porras and Plaza Martín have studied the group scheme of automorphisms of the field of Laurent series, they present this group (the Virasoro group) as a local generator for the moduli space of curves and, since the theory is independent of the genus of the curve, this allows them to formulate a non perturbative Conformal Field Theory ([34]). In the same spirit, the group scheme $\Gamma$ of invertible elements of a field of Laurent series (that appears in [3, 42]) uniformizes the Jacobian of a curve and allows to linearize certain flows in the Sato Grassmannian. This group scheme is the algebraic version of the loop group of $\mathcal{S}^{1}$.

In [31] soliton hierarchies appear naturally in the study of Hurwitz schemes. They use the trace map as a key tool to characterize the image of the Krichever map and to give concrete differential equations describing Hurwitz schemes, providing in addition a different approach to the problems of [1, 24]. Hurwitz spaces are spaces of finite coverings $Y \rightarrow X$ between (integral and smooth) curves, and thus, we can think of the field of fractions of $Y$ as an extension of the field of fractions of $X$, what allows to give a geometric point of view of the so called "Class Field Theory" and to carry over certain results to the Langlands Program (as a non commutative version of the "Class Field Theory"). In this direction there exists relevant and interesting results, among which one can specially mention the works by Frenkel and Ben-Zvi ([8, 15, 14, 13]), where moreover, one can confirm the usefulness of the tools that the infinite Grassmannian provide us.

Finally, another key ingredient for this work is the theory of Higgs pairs. Let us first explain why some people wanted to attack them from the point of view of the infinite Grassmannian. Let's get back in time until 1988, that year, and motivated by an abelianization (or linearization) of the study of vector bundles (what now is called Hitchin Abelianization Program), Hitchin ([21]) centered his research on the symplectic geometry of the cotangent space $\mathrm{T}^{*} \mathcal{U}_{X}$ to the moduli space $\mathcal{U}_{X}$ of vector bundles over a compact Riemann surface $X$, its points led him to introduce a new and currently relevant concept: the notion of Higgs pairs. From the point of view of sympletic geometry, the map from $\mathrm{T}^{*} \mathcal{U}_{X}$ to an affine space of global sections (now called the Hitchin map) turned out to be an algebraically completely integrable Hamiltonian system. This essentially means that the fibers of the Hitchin map are Jacobians of certain curves (the spectral curves). As the author states in [21], one question is left: can we find the Hamiltonian differential equations in some concrete way?

Bearing this question in mind, Li and Mulase partially solved the problem in [23] using techniques of infinite integrable systems. They used the infinite Sato Grassmannian as a 
space of solutions for the KP system ([40]) and answered the question of how to recover the Hitchin system from the KP system in the case on which the spectral cover is not ramified. In the same period, and with similar tools (Krichever morphism, Sato Grassmannian, ...), Donagi and Markman also studied this topic in [12], they proved certain compatibility for KP flows concerning spectral data coming from Higgs pairs on $X$.

We have differentiated four parts in this PhD Thesis. The first of them contains two chapters devoted to the study of certain group schemes and their Lie algebras. Some of these group schemes are generalizations of the Virasoro group ([34, 31]), some other are generalizations of the algebraic version of the loop group of $\mathcal{S}^{1}([42,3])$, and the remaining ones can be obtain as extensions of these group schemes. We should especially mention that these last group schemes appears as groups of semilinear automorphisms of certain fields of Laurent series, and their Lie algebras turn out to be algebras of scalar differential operators of order one. It is worth to remark that also the Weyl group can be thought of as a group of automorphisms of a field of Laurent series.

Second part includes three chapters (from 3 to 5), on which a systematic study of the Krichever map is made for different moduli spaces. First of these chapters is concerned with the moduli space of curves and vector bundles (both are allow to vary). We give a Krichever morphism for this moduli space (without admitting local structure of cyclic covering around the marked point, complementing the results of Mulase, [28]) and endow this moduli space with a scheme structure inside a Sato Grassmannian. We obtain a Mulase-like characterization of the image of the Krichever map only in terms of the point in the infinite Grassmannian defined by the vector bundle; chapter 4 studies the moduli space of Higgs pairs over a smooth fixed curve (with formal trivialization data). The infinite Grassmannian we have needed to define the Krichever map is a fibration of infinite Grassmannians over an affine space, which is nothing but a formal analogue of the so called Hitchin base. We prove that the moduli is representable, that the Krichever map is injective and we also characterize its image; and, in chapter 5 we show similar results for the moduli space of finite coverings (between smooth curves) and the moduli spaces of line bundles over such a covering maps, and we also offer an integrable system which behaves in a similar way to that of Hitchin, and study some of its properties by terms of the Hitchin Abelianization Program.

The third part gives an infinitesimal study of the moduli spaces on which we have center the work. It has two different chapters (6 and 7). First of them is oriented to compute the tangent space to the moduli spaces, doing the calculations from two points of view: using classical techniques of cohomology and hypercohomology, and using the algebraic tools that the Krichever map suggest us (which are very easy to handle with). We justify in this chapter the study of the group schemes and their Lie algebras made in the first part, because it turns out that these group schemes play the role of local generators for the moduli spaces (in the sense that they act on the moduli and the orbit morphism is surjective at the level of tangent spaces). In chapter 7 we offer the last result of the work: concrete computations for hierarchies of differential equations describing the moduli space of Higgs pairs with formal trivialization data.

Finally, we have include a part containing appendices. First of them is devoted to the 
construction of the algebraic Sato Grassmannian, second is oriented to detail the inverse construction of the Krichever map for the moduli space of curves, and the third one, which summarizes general results about the theory of differential operators given by Grothendieck.

Next sections will summarize the contents of this work. For the sake of simplicity, we will omit the proofs and we will mostly work with rational points.

\section{D.1. Group schemes.}

In this section we study certain group schemes. It turns out that these groups will play, in section D.6, the role of local generators for moduli spaces. Some of these group schemes are generalizations of the Virasoro group ([34, 31]), some others are generalizations of the algebraic version of the loop group of $\mathcal{S}^{1}([42,3])$, and the remaining ones can be obtain as extensions of these group schemes. We should especially mention that these last group schemes appear as groups of semilinear automorphisms of certain fields of Laurent series.

Let $k$ be an algebraically closed field of characteristic zero. In [3] it is proved that the following functor is representable by a formal $k$-scheme:

$$
\begin{aligned}
& \underline{k((z))^{*}}: \mathcal{C}_{k-e s q} \rightarrow \mathcal{C}_{g r p} \\
& S \mapsto \underline{k((z))^{*}}(S)=\mathrm{H}^{0}\left(S, \mathcal{O}_{S}\right)((z))^{*}
\end{aligned}
$$

where for any $k$-algebra $R, R((z))^{*}$ denotes the group of invertible elements of the ring $R[[z]]\left[z^{-1}\right]$.

Let us write $\Gamma$ for its connected component of the origin (its group law is multiplication of Laurent series). This group is the algebraic version of the loop group of $\mathcal{S}^{1}$. One has a decomposition:

$$
\Gamma=\Gamma^{-} \times \mathbb{G}_{m} \times \Gamma^{+}
$$

and any $\gamma \in \Gamma(R)$ is written by $\gamma=\left(\gamma_{-}, \gamma_{0}, \gamma_{+}\right)$where:

$$
\gamma_{-}=1+\sum_{j=-m}^{-1} a_{j} z^{j} \text { with } a_{j} \in \operatorname{Rad}(R) ; \gamma_{0} \in R^{*} ; \gamma_{+}=1+\sum_{j \geq 1} a_{j} z^{j} \text { with } a_{j} \in R
$$

Analogously, if one is given a separable $k((z))$-algebra $V$ of dimension $n$ joint with a decomposition into irreducible $k((z))$-algebras:

$$
V \simeq V_{1} \times \cdots \times V_{r}
$$

one has (see [31]):

$$
\Gamma_{V}=\Gamma_{V_{1}} \times \cdots \times \Gamma_{V_{r}} \quad \text { where } \quad \Gamma_{V_{i}}=\Gamma_{V_{i}}^{-} \times \mathbb{G}_{m} \times \Gamma_{V_{i}}^{+}
$$

Another relevant group we should mention here is the group of $k$-algebra isomorphisms defined by:

$$
\operatorname{Spec} k \rightarrow \mathrm{G}_{V_{i}}(k):=\operatorname{Aut}_{k-a l g} V_{i}
$$


where the group law is given by composing automorphisms. Given a separable $k((z))$ algebra $V$ of dimension $n$, it can be checked (see [34, 32]) that the group whose rational points are given by:

$$
\mathrm{G}_{V}(k):=\operatorname{Aut}_{k-a l g} V
$$

is representable by the group $k$-scheme $G_{V}=\left(G_{V_{1}} \times \cdots \times G_{V_{r}}\right) \rtimes \mathcal{S}_{r}$, where $\mathcal{S}_{r}$ is the symmetric groups of $r$ letters and acts on $\mathrm{G}_{V_{1}} \times \cdots \times \mathrm{G}_{V_{r}}$ by the formula:

$$
\left(\sigma,\left(g_{1}, \ldots, g_{r}\right)\right) \mapsto\left(g_{\sigma(1)}, \ldots, g_{\sigma(r)}\right) .
$$

These groups are generalizations of the Virasoro group.

The $k((z))$-algebra structure of $V, \pi: k((z)) \rightarrow V$, allow us to define the subgroup $\mathrm{G}_{k((z))}^{V}$ of $\mathrm{G}_{V}$ as the one whose elements are automorphisms $\bar{g} \in \mathrm{G}_{V}$ making the following diagram commutative:

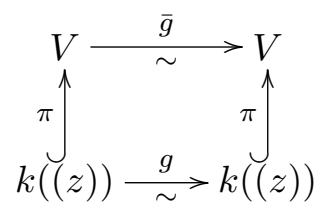

where $g \in \mathrm{G}_{k((z))} \stackrel{\text { not }}{=} \mathrm{G}$. We write $\bar{g}$ for the element of $\mathrm{G}_{k((z))}^{V}$ given by the diagram above. This group is slightly different from that of [32, Sec.5], there it is defined as a subgroup of the connected component of the origin of $\mathrm{G}_{V}$, thus loosing the combinatorics of the group $\mathcal{S}_{r}$ that we will need to make use of later. Moreover, in [32] a explicit $k((z))$-algebra structure on $V$ is fixed, and we want this structure to vary. Let us briefly explain what this means.

From now on let us assume that $V$ is a separable $k((z))$-algebra of dimension $n$, let us fix a partition $\underline{n}=\left(n_{1}, \ldots, n_{r}\right)$ (where $n_{1}+\cdots+n_{r}=n$ ) and let us state the following notations:

- Denote $V_{\underline{n}}$ for the product $k\left(\left(z_{1}\right)\right) \times \cdots \times k\left(\left(z_{r}\right)\right)$ with the following $k((z))$-algebra structure:

$$
\begin{aligned}
k((z)) & \rightarrow k\left(\left(z_{1}\right)\right) \times \cdots \times k\left(\left(z_{r}\right)\right) \\
z & \mapsto\left(z_{1}^{n_{1}}, \ldots, z_{r}^{n_{r}}\right)
\end{aligned}
$$

(this is the one used in [32, Section 5.4]). For the shake of simplicity, the group $\mathrm{G}_{k((z))}^{V_{n}}$ will be denoted by $\mathrm{G}_{\underline{n}}$.

- Let $p(T) \in k[[z]][T]$ be a degree $n$ monic polynomial in such a way that its decomposition into irreducible factors is:

$$
p(T)=p_{1}(T) \cdots p_{r}(T), \text { where } p_{i}(T)=T^{n_{i}}-z u_{i}(z, T)
$$

and $u_{i}(z, T) \in k[[z]][T]$ are polynomial of degree $<n_{i}$ such that there exists $u_{i}(z, T)^{-1}$ and $u_{i}(0,0)=1$. 
Denote $V_{\underline{n}, p}$ for the product $\prod_{i=1}^{r} k((z))[T] / p_{i}(T)$ with the following $k((z))$-algebra structure:

$$
\begin{aligned}
k((z)) & \rightarrow k((z))[T] / p_{1}(T) \times \cdots \times k((z))[T] / p_{r}(T) \\
z & \mapsto\left(T^{n_{1}} u_{1}(z, T)^{-1}, \ldots, T^{n_{r}} u_{r}(z, T)^{-1}\right)
\end{aligned}
$$

(these decompositions are motivated by geometric facts, see Proposition 5.3. For the shake of simplicity, the group $\mathrm{G}_{k((z))}^{V_{n, p}}$ will be denoted by $\mathrm{G}_{\underline{n}, p}$.

- We keep the notation $V$ for the cases on which, either it is no necessary to specify the $k((z))$-algebra structure of $V$, either it is irrelevant for the computations.

Proposition D.1. (Proposition 1.10) One has a natural isomorphism of group schemes:

$$
\mathrm{G}_{\underline{n}} \simeq \mathrm{G}_{\underline{n}, p} .
$$

Remark D.2. For points with values in a $k$-scheme $S$, we need to endow the category of $k$-schemes with the étale topology to make this result work.

Let $\operatorname{Tr}: V \rightarrow k((z))$ the trace map of $V$ as a finite $k((z))$-algebra. One can prove the following result:

Proposition D.3. (Proposition 1.12) Let $\bar{g}$ be an element in $\mathrm{G}_{V}$. Then:

$$
\bar{g} \in \mathrm{G}_{k((z))}^{V} \Longleftrightarrow \operatorname{Tr} \circ \bar{g}=g \circ \mathrm{Tr}
$$

This result generalizes Lemma 5.8 of [32], since it allows the $k((z))$-algebra structure of $V$ to vary. The key ingredient to prove it consists of making use of the Newton-Girard identities. 1.14):

It is worth pointing out that there exists an exact sequence of groups (see Proposition

$$
0 \longrightarrow \operatorname{Aut}_{k((z))-\text { alg }} V \longrightarrow \mathrm{G}_{k((z))}^{V} \stackrel{p}{\longrightarrow} \mathrm{G} \longrightarrow 0
$$

where $p(\bar{g})=g$. For the case of $V_{\underline{n}}$ we also have that:

$$
\operatorname{Aut}_{k((z))-\text { alg }} V_{\underline{n}} \simeq \mu_{\underline{n}} \rtimes \mathcal{S}_{\underline{n}}, \text { where } \mathcal{S}_{\underline{\underline{n}}}=<\left\{(i, j) \in \mathcal{S}_{r} \mid n_{i}=n_{j}\right\}>.
$$

Let us now introduce the Heisenberg group and the Weyl group. The action by homoteties of $V^{*}$ (invertible elements) on $V$, induces a natural embedding:

$$
V^{*} \hookrightarrow \mathrm{Gl}_{k((z))} V,
$$

where $\mathrm{Gl}_{k((z))} V$ is the group of $k((z))$-linear automorphisms of $V$.

We define the Heisenberg group of $V$, denoted by Heis $V$, as the image of $\Gamma_{V}$ by the embedding above. The Weyl group is defined by the quotient:

$$
\mathcal{W}=\mathrm{N}_{k((z))}\left(\operatorname{Heis}_{V}\right) / \text { Heis }_{V}
$$

$\mathrm{N}_{k((z))}\left(\right.$ Heis $\left._{V}\right)$ being the normalizer of $\operatorname{Heis}_{V}$ inside $\mathrm{Gl}_{k((z))}(V)$. One has the following result: 
Theorem D.1.1. (Theorem 1.19)

1. The following sequence is exact:

$$
0 \rightarrow \operatorname{Heis}_{V} \rightarrow \mathrm{N}_{k((z))}\left(\operatorname{Heis}_{V}\right) \rightarrow \operatorname{Aut}_{k((z))-a l g} V \rightarrow 0
$$

2. $\mathcal{W} \simeq \operatorname{Aut}_{k((z))-a l g} V$.

3. The action by conjugation of $\operatorname{Aut}_{k((z))-a l g} V$ on Heis $V$ induces an isomorphism:

$$
\mathrm{N}_{k((z))}\left(\text { Heis }_{V}\right) \simeq \text { Heis }_{V} \rtimes \mathcal{W}
$$

Note that the result introduces the Weyl group as an automorphism group of $V$. Now, we are about to define the group schemes of semilinear automorphisms.

Definition D.4. We define the group $\operatorname{SGl}_{k((z))}(V)$ of semilinear automorphisms of $V$ as the group of $k$-linear automorphisms:

$$
\gamma: V \simeq V
$$

for which there exists a $k$-algebra automorphism of $k((z)), g \in \mathrm{G}$, in such a way that:

$$
\gamma(z \cdot v)=g(z) \cdot \gamma(v)
$$

Proposition D.5. (Proposition 1.21) The following sequence is an exact sequence of groups:

$$
0 \rightarrow \mathrm{Gl}_{k((z))}(V) \rightarrow \mathrm{SGl}_{k((z))}(V) \rightarrow \mathrm{G} \rightarrow 0
$$

in such a way that the action $\mathrm{G}$ on $\mathrm{Gl}_{k((z))}(V)$ induces an isomorphism:

$$
\operatorname{SGl}_{k((z))}(V) \simeq \mathrm{Gl}_{k((z))}(V) \rtimes \mathrm{G}
$$

In particular, for $V=k((z))$ the group $\operatorname{SGl}_{k((z))}(k((z)))$ is exactly the group $\Gamma \rtimes \mathrm{G}$ used in [32, Section 6].

In a similar fashion, we define:

Definition D.6. The group $\mathrm{SHeis}_{V}$ is the subgroup of $\operatorname{SGl}_{k((z))}(V)$ given by those $k$-linear automorphisms:

$$
\gamma: V \simeq V
$$

for which there exists a $k$-algebra automorphism $g \in \mathrm{G}_{V}$, and an element $w \in V^{*}$ such that:

$$
\gamma(v)=w \cdot g(v)
$$

Note that $w=\gamma(1) \in V^{*}$.

Proposition D.7. (Proposition 1.24) The following sequence is an exact sequence of groups:

$$
0 \rightarrow \text { Heis }_{V} \rightarrow \text { SHeis }_{V} \rightarrow \mathrm{G}_{V} \rightarrow 0
$$

and the action of $\mathrm{G}_{V}$ on $\mathrm{Heis}_{V}$ produces:

$$
\text { SHeis }_{V} \simeq \text { Heis }_{V} \rtimes \mathrm{G}_{V}
$$


Moreover, one has that the natural embedding $\mathrm{G}_{V} \hookrightarrow \mathrm{N}_{k}\left(\mathrm{Heis}_{V}\right)$ induces the following isomorphism:

$$
\mathrm{N}_{k}\left(\text { Heis }_{V}\right) / \text { Heis }_{V} \simeq \mathrm{G}_{V},
$$

(where $\mathrm{N}_{k}\left(\right.$ Heis $\left._{V}\right)$ is the normalizer of $\operatorname{Heis}_{V}$ inside $\mathrm{Gl}_{k}(V)$ ) and:

$$
\text { SHeis }_{V} \simeq \mathrm{N}_{k}\left(\text { Heis }_{V}\right)
$$

The next group will make use of the $k((z))$-algebra structure of $V$.

Definition D.8. The group $\operatorname{SHeis}_{k((z))}^{V}$ is the group of $k$-linear automorphisms:

$$
\gamma: V \simeq V
$$

for which there exists $\bar{g} \in \mathrm{G}_{k((z))}^{V}$ and $w \in V^{*}$ in such a way that:

$$
\gamma(v)=w \cdot \bar{g}(v)
$$

Proposition D.9. (Proposition 1.28) One has an exact sequence of groups:

$$
0 \rightarrow \operatorname{Heis}_{V} \rightarrow \operatorname{SHeis}_{k((z))}^{V} \rightarrow \mathrm{G}_{k((z))}^{V} \rightarrow 0
$$

where the action of $\mathrm{G}_{k((z))}^{V}$ on $\mathrm{Heis}_{V}$ induces:

$$
\operatorname{SHeis}_{k((z))}^{V} \simeq \operatorname{Heis}_{V} \rtimes \mathrm{G}_{k((z))}^{V}
$$

\section{D.2. Lie algebras of the group schemes.}

Thinking of the Lie algebra of a group scheme as the tangent space at the identity, direct computations show the following results:

$$
\text { Lie Heis }_{V} \simeq V, \operatorname{LieGl}_{k((z))}(V) \simeq \operatorname{End}_{k((z))} V \text { and } \operatorname{LieG}_{V} \simeq \operatorname{Der}_{k}(V, V) .
$$

Bearing in mind the exact sequence:

$$
0 \rightarrow \operatorname{Aut}_{k((z))-a l g} V \rightarrow \mathrm{G}_{k((z))}^{V} \rightarrow \mathrm{G} \rightarrow 0
$$

and the fact that $\operatorname{Aut}_{k((z))-a l g} V$ is a discrete group, we obtain that $\operatorname{Lie}_{k((z))} \stackrel{\longrightarrow}{\longrightarrow}$ Lie G. Moreover, it can be shown that one can gets the following Lie algebra isomorphisms:

$$
\operatorname{Lie~}_{k((z))}^{V} \simeq \operatorname{Der}(V, V)^{\pi} \simeq \operatorname{Der}(V, V)^{\operatorname{Tr}} \simeq \text { Lie G },
$$

where $\operatorname{Der}(V, V)^{\pi}$ consist of those derivations $\theta_{V} \in \operatorname{Der}_{k}(V, V)$ for which there exist a derivation $\theta \in \operatorname{Der}_{k}(k((z)), k((z)))$ verifying $\theta_{V} \circ \pi=\pi \circ \theta$ (where $\pi: k((z)) \rightarrow V$ 
is the $k((z))$-algebra structure of $V)$. Similarly, we write $\operatorname{Der}(V, V)^{\operatorname{Tr}}$ for the derivations compatible with the trace map $\operatorname{Tr}: V \rightarrow k((z))$ of $V$ as a finite $k((z))$-algebra.

For the case of the groups of semilinear automorphisms, let us recall the natural exact sequence of scalar differential operators:

$$
0 \rightarrow \operatorname{End}_{k((z))} V \rightarrow \mathcal{D}_{k((z)) / k}^{1}(V, V) \stackrel{\sigma}{\rightarrow} \operatorname{Der}_{k}(k((z))) \rightarrow 0 .
$$

Then, one can prove that there exists a natural isomorphism of Lie algebras:

$$
\operatorname{Lie}\left(\operatorname{SGl}_{k((z))}(V)\right) \simeq \mathcal{D}_{k((z)) / k}^{1}(V, V) .
$$

In a similar way, we can obtain Lie algebra isomorphisms:

$$
\begin{aligned}
\operatorname{Lie}\left(\operatorname{SHeis}_{V}\right) & \simeq \mathcal{D}_{V / k}^{1}(V, V) \\
\operatorname{Lie}\left(\operatorname{SHeis}_{k((z))}^{V}\right) & \simeq \mathcal{D}_{V / k}^{1}(V, V)^{\pi}
\end{aligned}
$$

where $\mathcal{D}_{V / k}^{1}(V, V)^{\pi}$ consist of those differential operators in $\mathcal{D i f f}_{V / k}^{1}(V, V)$ whose symbol morphism ( $\sigma$ below) takes values in $\operatorname{Der}_{k}(V, V)^{\pi}$.

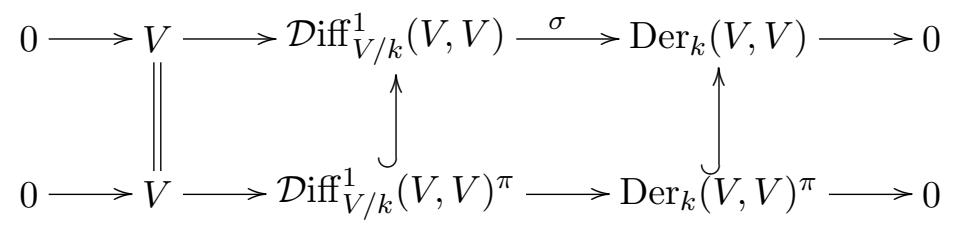

\section{D.3. Moduli of curves and vector bundles.}

Consider the moduli space $\mathcal{U}^{\infty}$ of data $(X, p, z, E, \phi)$ where $X$ is a smooth, integral, projective curve over an algebraically closed field $k$ of characteristic zero, $p \in X, z$ local parameter, $E$ is a rank $n$ vector bundle on $X$ and $\phi$ is formal trivialization of $E$ at $p$. The Krichever morphism ([28], [30], [1], [33], ...) allows us to send these data to the point $W=\mathrm{H}^{0}(X-p, E)$ of the infinite Grassmannian of $k((z))^{n}, \operatorname{Gr}\left(k((z))^{n}\right)$. It turns out that this morphism is an embedding so we can characterize its image. We also define a Krichever morphism taking values in a couple of infinite Grassmannians and, since the infinite Grassmannian is representable by a scheme ([3]), we can prove that also $\mathcal{U}^{\infty}$ is representable. Similar results can also be obtained for the case on which there exists singular curves on the moduli.

Rational points of $\mathcal{U}^{\infty}$ correspond to data $(X, p, z, E, \phi)$ where $X$ is a smooth curve with marked point $p \in X, z$ is a formal parameter on $p$, that is:

$$
\widehat{\mathcal{O}}_{X, p} \simeq k[[z]],
$$


$E$ is a rank $n$ vector bundle on $X$ and $\phi$ is a $\widehat{\mathcal{O}}_{X, p}$-module isomorphism:

$$
\widehat{E}_{p} \simeq \widehat{\mathcal{O}}_{X, p}^{n}
$$

The Krichever morphism is define as follows:

$$
\begin{aligned}
\mathrm{Kr}: \mathcal{U}^{\infty} & \rightarrow \operatorname{Gr}\left(k((z))^{n}\right) \\
(X, D, z, E, \phi) & \mapsto(z \circ \phi) H^{0}(X-p, E)
\end{aligned}
$$

Let us denote $W=(z \circ \phi) H^{0}(X-p, E)$. We are going to show that $W$ is indeed a point of:

$$
\operatorname{Gr}\left(k((z))^{n}\right)(k)=\left\{\begin{array}{c}
W \subseteq k((z))^{n} \text { such that } W \cap k[[z]]^{n} \text { and } k((z))^{n} / W+k[[z]]^{n} \\
\text { are finite dimensional over } k
\end{array}\right\}
$$

Note that sending a section of $\mathrm{H}^{0}(X-p, E)$ to its germ at $p$ (that is, using $z$ and $\phi$ ) we have an inclusion:

$$
W=H^{0}(X-p, E) \hookrightarrow\left(\widehat{\mathcal{O}}_{X, p}\right)_{0}^{n} \simeq k((z))^{n} .
$$

Consider the exact sequence of sheaves:

$$
0 \rightarrow E \rightarrow E(m p) \rightarrow\left(\mathcal{O}_{m p}(m p)\right)^{n} \rightarrow 0
$$

and take cohomology:

$$
0 \rightarrow \mathrm{H}^{0}(X, E) \rightarrow \mathrm{H}^{0}(X, E(m p)) \rightarrow\left(\mathcal{O}_{m p}(m p)\right)^{n} \rightarrow \mathrm{H}^{1}(X, E) \rightarrow \mathrm{H}^{1}(X, E(m p)) \rightarrow 0
$$

where the last 0 is deduced because $\mathcal{O}_{m p}(m p)$ is flabby (it is concentrated at $p$ ). Moreover, for $m$ bigger enough $\mathrm{H}^{1}(X, E(m p))=0$, so:

$$
0 \rightarrow \mathrm{H}^{0}(X, E) \rightarrow \mathrm{H}^{0}(X, E(m p)) \rightarrow\left(\mathcal{O}_{m p}(m p)\right)^{n} \rightarrow \mathrm{H}^{1}(X, E) \rightarrow 0
$$

Since $\mathcal{O}_{m p}(m p)$ is the quotient between meromorphic functions on $X$ with possibly a pole at $p$ of order at worst $m$ and regular functions on $X$, it follows that:

$$
\mathcal{O}_{m p}(m p)=\left\langle z^{-1}, \ldots, z^{-m}\right\rangle
$$

Bearing in mind that:

- $\lim _{\longrightarrow} \mathcal{O}_{m p}(m p)=k((z)) / k[[z]]$

- $\lim _{\longrightarrow} \mathrm{H}^{0}(X, E(m p))=\mathrm{H}^{0}(X-p, E)=W$,

and taking $\underline{\lim }_{m}$ in the sequence $(\mathrm{D} .3 .1)$ we get the following exact sequence:

$$
0 \rightarrow \mathrm{H}^{0}(X, E) \rightarrow W \rightarrow k((z))^{n} / k[[z]]^{n} \rightarrow \mathrm{H}^{1}(X, E) \rightarrow 0
$$


Since $\mathrm{H}^{0}(X, E)$ and $\mathrm{H}^{1}(X, E)$ are finite dimensional $k$-vector spaces, we conclude:

$$
W=\mathrm{H}^{0}(X-p, E) \in \operatorname{Gr}\left(k((z))^{n}\right)(k) .
$$

Let us write:

$$
A_{W}:=\{f \in k((z)) \mid f \cdot W \subseteq W\}
$$

for the stabilizer algebra of $W$ in $k((z))$. The image of the Krichever map is characterized by the following result.

Theorem D.3.1. (Theorem 3.19) A rational point $W \in G r(V)$ lies on the image of the Krichever morphism for $\mathcal{U}^{\infty}$ if and only if $A_{W} \in G r(k((z))), k \subset A_{W}$ and it is regular. In particular, the Krichever morphism is injective.

Now we define a second Krichever morphism in terms of two Grassmannians.

Definition D.10. (Definition 3.5) We define the Krichever morphism by:

$$
\begin{aligned}
\mathrm{Kr}_{2}: \mathcal{U}^{\infty} & \rightarrow \operatorname{Gr}\left(k((z))^{n}\right) \times \operatorname{Gr}(k((z))) \\
(X, D, z, E, \phi) & \mapsto\left((z \circ \phi) H^{0}(X-p, E), z H^{0}\left(X-p, \mathcal{O}_{X}\right)\right)
\end{aligned}
$$

It is an injective map and its image is characterized by the following result.

Theorem D.3.2. (Theorem 3.20 A couple of rational points $(W, A) \in G r(V) \times \operatorname{Gr}(k((z)))$ lies in the image of the Krichever morphism $\mathrm{Kr}_{2}$ if and only if $A$ is a non-trivial regular sub- $k$-algebra of $k((z))$ and $W$ is $A$-module.

Remark D.11. For points with values in any $k$-scheme $S$ some technical details concerning the stabilizer algebra are needed.

Following the ideas of [2, 33, 34] we can say that:

Theorem D.3.3. (Theorem 3.22) The functor $\mathcal{U}^{\infty}$ is representable by a locally closed subscheme of $\operatorname{Gr}(V) \times \operatorname{Gr}(k((z)))$.

Similar results also hold for the case on which the curve could be singular (but the marked point is still forced to be a non-singular one).

\section{D.4. Moduli of Higgs Pairs.}

In this section, we summarize the results of chapter 4 . The moduli space of Higgs pairs over a fixed smooth projective curve with extra formal data is defined and is endowed with a scheme structure. We introduce a relative version of the Krichever map using a fibration of Sato Grassmannians and show that this map is injective. This, together with the characterization of the points of the image of the Krichever map, allows us to prove that this moduli space is a closed subscheme of the product of the moduli of vector bundles (with formal extra data) and a formal analogue of the Hitchin base. This characterization also provides us 
with a method for explicitly computing KP-type equations that describe the moduli space of Higgs pairs in the last chapter.

Let us first offer a few words about the classical correspondence between Higgs pairs and line bundles over the spectral curve. Recall that a Higgs pair over a sooth curve $X$ consists of a vector bundle $E \in \mathcal{U}_{X}$ and a morphism of sheaves of $\mathcal{O}_{X}$-modules:

$$
\varphi: E \rightarrow E \otimes \omega_{X}
$$

It is known ([12]) that:

$$
\mathrm{T}_{E}^{*} \mathcal{U}_{X} \simeq \mathrm{H}^{0}\left(X, \mathcal{E n d}_{X} E \otimes \omega_{X}\right)
$$

and therefore, the cotangent space to $\mathcal{U}_{X}$ parametrizes Higgs pairs on $X$.

A remarkable fact is the existence of the Hitchin map:

$$
\begin{aligned}
H: \mathrm{T}^{*} \mathcal{U} & \rightarrow \oplus_{i=1}^{n} \mathrm{H}^{0}\left(X, \omega_{X}^{\otimes i}\right) \\
(E, \varphi) & \mapsto \operatorname{ch}(\varphi),
\end{aligned}
$$

where $\operatorname{ch}(\varphi)=\left(\operatorname{Tr}(\varphi), \operatorname{Tr}\left(\Lambda^{2} \varphi\right), \ldots, \operatorname{Tr}\left(\Lambda^{n} \varphi\right)\right)$ are the characteristic coefficients of $\varphi$.

Another relevant issue is the spectral construction, whose importance lies in the fact that, for a generic $a=\left(a_{1}, \ldots, a_{n}\right) \in \oplus_{i=1}^{n} \mathrm{H}^{0}\left(X, \omega_{X}^{\otimes i}\right)$, the points of $H^{-1}(a)$ correspond to line bundles on the so called spectral curve $X_{a}$.

We will now define the moduli of Higgs pairs with formal extra data and the appropriate Krichever map. We shall assume that the following data are definitively fixed:

- $X$ is a smooth, projective, integral curve over $k$ (algebraically closed of characteristic zero).

- $x \in X$.

- $t: \widehat{\mathcal{O}}_{X, x} \simeq k[[z]]$ is a formal trivialization of $X$ at $x$.

Since $X$ is smooth, the dualizing sheaf $\omega_{X}$ is a line bundle, and the formal trivialization $t$ induces a formal trivialization of $\omega_{X}$ at $x$.

We define the moduli space of Higgs pairs with formal extra data as folllows. Let us denote by $\widehat{\varphi}$ the completion at $x$ of the Higgs field $\varphi$. Given the formal trivialization $\phi$, the coefficients $a_{i}$ of the characteristic polynomial $p_{\hat{\varphi}}(T)$ of $\widehat{\varphi}$ lie on $k[[z]]$.

We will write $\mathcal{H i g g s}_{X}^{\infty}$ for the moduli space of Higgs pairs over $X$ with formal extra data. Its rational points are given by the following data $(E, \varphi, \phi)$ :

1. $E$ is a rank $n$ vector bundle over $X$.

2. $\varphi: E \rightarrow E \otimes \omega_{X}$ is a Higgs field; that is, a morphism of sheaves of $\mathcal{O}_{X}$-modules.

3. $\phi$ is a formal trivialization of $E$ along $x$ :

$$
\phi: \widehat{E}_{x} \simeq \widehat{\mathcal{O}}_{x}^{\oplus n},
$$


such that there exists a matrix:

$$
M=\left(\begin{array}{cccccc}
0 & \cdot & & \cdot & 0 & (-1)^{n+1} a_{n} \\
1 & 0 & \cdot & & \cdot & (-1)^{n} a_{n-1} \\
0 & 1 & 0 & \cdot & \cdot & \cdot \\
\cdot & \cdot & \cdot & \cdot & \cdot & \cdot \\
\cdot & \cdot & 0 & 1 & 0 & -a_{2} \\
0 & \cdot & \cdot & 0 & 1 & a_{1}
\end{array}\right)
$$

(where $\left(a_{1}, \ldots, a_{n}\right) \in k[[z]]^{n}$ ) making the following diagram commutative:

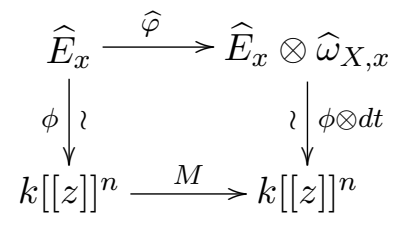

4. Two triples $(E, \varphi, \phi)$ and $\left(E^{\prime}, \varphi^{\prime}, \phi^{\prime}\right)$ are said to be equivalent whenever there exists an isomorphism of vector bundles $f: E \simeq E^{\prime}$ compatible with all data. That is:

- $f$ is compatible with $\phi$ and $\phi^{\prime}$, and hence the following diagram is commutative:

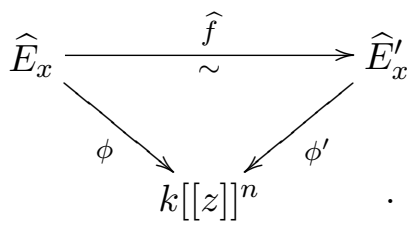

- $f$ is compatible with $\varphi$ and $\varphi^{\prime}$; that is, the diagram:

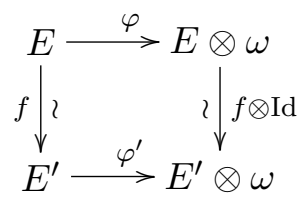

is commutative.

Proposition 4.4 shows that this moduli is non empty and Theorem 4.5 proves that it is representable.

We have already stated that the coefficients $a_{i}$ of the characteristic polynomial $p_{\widehat{\varphi}}(T)$ of $\widehat{\varphi}$ lie on $k[[z]]$. Let us now introduce a $k$-scheme parametrizing these coefficients. Let $\mathbb{A}^{\infty}$ be the infinite dimensional affine scheme over $k$ defined in [3, Def. 4.13]:

$$
\mathbb{A}^{\infty}:=\operatorname{Spec} \underset{l}{\lim } k\left[y_{0}, \ldots, y_{l}\right]=\operatorname{Spec} k\left[y_{0}, y_{1}, \ldots\right]
$$


Let $R$ be a $k$-algebra. The correspondence:

$$
s_{0}+s_{1} z+\cdots \in R[[z]] \leftrightarrow\left(s_{0}, s_{1}, \ldots\right) \in \mathbb{A}^{\infty}(R)
$$

implies that the functor that associates the ring of formal power series $R[[z]]$ with each $k$ algebra, $R$, is representable by $\mathbb{A}^{\infty}$. From this fact we obtain the following:

Proposition D.12. (Proposition 4.6) The functor over the category of $k$-schemes defined by:

$$
S \rightsquigarrow \oplus_{i=1}^{n} \mathrm{H}^{0}\left(\widehat{X}_{x} \times S, \widehat{\omega}_{x \times S}^{\otimes i}\right)
$$

is representable by the $k$-scheme $\mathbb{A}=\left(\mathbb{A}^{\infty}\right)^{n}$.

We shall think of its rational points as polynomials:

$$
T^{n}-a_{1} T^{n-1}+\cdots+(-1)^{n} a_{n},
$$

where $a_{i} \in k[[z]]$ vía the identification $\widehat{\omega}_{X, x}^{\otimes i} \simeq k[[z]]$ induced by $t$. Now, the formal Hitchin map is the morphism of schemes:

$$
\begin{aligned}
\mathcal{H}_{\infty}: \mathcal{H i g g s}_{X}^{\infty} & \rightarrow \mathbb{A} \\
(E, \phi, \varphi) & \mapsto\left(a_{1}, \ldots, a_{n}\right)
\end{aligned}
$$

where $\left(a_{1}, \ldots, a_{n}\right)$ are the characteristic coefficients $a_{i}=\operatorname{Tr}\left(\Lambda^{i} \widehat{\varphi}\right)$ of the formal Higgs field $\widehat{\varphi}$.

Let us introduce the Krichever map for $\operatorname{Higgs}_{X}^{\infty}$. Let $(E, \phi, \varphi)$ be a rational point of $\mathcal{H}_{\text {iggs }}{ }^{\infty}$. The Krichever map is defined by:

$$
\begin{aligned}
\mathcal{H i g g s}_{X}^{\infty} & \rightarrow \operatorname{Gr}\left(k((z))^{n}\right) \times \mathbb{A} \\
(E, \phi, \varphi) & \mapsto\left((t \circ \phi) \mathrm{H}^{0}(X-x, E), p_{\widehat{\varphi}}(T)\right),
\end{aligned}
$$

where $p_{\widehat{\varphi}}(T)$ is the characteristic polynomial of the formal Higgs field $\widehat{\varphi}$. Theorem 4.10 shows that this map is injective.

In order to characterize its image, notice that $([2,28])$ the image of the Krichever map for vector bundles:

$$
\mathcal{U}_{X}^{\infty} \hookrightarrow \operatorname{Gr}(V)
$$

consists of those points $W \in \operatorname{Gr}(V)$ such that $A \cdot W \subseteq W$ (where $A=\mathrm{H}^{0}\left(X-x, \mathcal{O}_{X}\right)$, see [33]). Since:

$$
\mathcal{H i g g s}_{X}^{\infty} \hookrightarrow \operatorname{Gr}(V) \times \mathbb{A}
$$

takes values in $\mathcal{U}_{X}^{\infty} \times \mathbb{A}$, we are interested in studying the image of:

$$
\mathcal{H i g g s}_{X}^{\infty} \hookrightarrow \mathcal{U}_{X}^{\infty} \times \mathbb{A}
$$

The image is characterized by the following theorem. 
Theorem D.4.1. (Theorem 4.15 Let $\Omega$ be the rational point in $\operatorname{Gr}(k((z)))$ defined by the canonical sheaf $\omega_{X}$, let $(W, p(T))$ be a rational point of $\mathcal{U}_{X}^{\infty} \times \mathbb{A}$, and let $T$ be the $k((z))$ linear automorphism of $k((z))^{n}$ given by the matrix:

$$
T=\left(\begin{array}{cccccc}
0 & \cdot & & \cdot & 0 & (-1)^{n+1} a_{n} \\
1 & 0 & \cdot & & \cdot & (-1)^{n} a_{n-1} \\
0 & 1 & 0 & \cdot & \cdot & \cdot \\
\cdot & \cdot & \cdot & \cdot & \cdot & \cdot \\
\cdot & \cdot & 0 & 1 & 0 & -a_{2} \\
0 & \cdot & \cdot & 0 & 1 & a_{1}
\end{array}\right)
$$

(where $a_{i}$ are the coefficients of $p(T)$ ). We have that, $(W, p(T))$ lies on the image of the Krichever map:

$$
\mathcal{H i g g s}_{X}^{\infty} \hookrightarrow \mathcal{U}_{X}^{\infty} \times \mathbb{A}
$$

if and only if $T(W) \subseteq W \cdot \Omega$.

Moreover, this allow us to state that $\mathcal{H} i g g s_{X}^{\infty}$ is a closed subscheme of $\mathcal{U}_{X}^{\infty} \times \mathbb{A}$ (Theorem 4.16 .

To finish with, let us briefly describe the "formal" analogue to the spectral construction. We refer to $\operatorname{Spf} k[[z]]$ as the formal base curve, and given a rational point $p(T) \in \mathbb{A}$, we define the formal spectral curve by:

$$
\operatorname{Spf} k[[z]][T] / p(T) .
$$

One can prove that, given a rational point $(E, \phi, \varphi) \in \mathcal{H i g g s}_{X}^{\infty}, \phi$ induces a formal trivialization of $E$ as a line bundle over the spectral curve $\pi: X_{\varphi} \rightarrow X$ :

$$
\phi_{\varphi}: \widehat{E}_{x} \simeq k[[z]][T] / p_{\widehat{\varphi}}(T),
$$

such that the matrix of Definition 4.1 is the multiplication by $T$ in $k[[z]][T] / p_{\widehat{\varphi}}(T)$ w.r.t the basis $\left\{1, T, \cdots, T^{n-1}\right\}$.

Using this formal trivialization it is easy to check that:

$$
W=\phi_{\varphi} \mathrm{H}^{0}\left(X_{\varphi}-\pi^{-1}(x), E\right)
$$

is a rational point of the infinite Grassmannian $\operatorname{Gr}\left(k((z))[T] / p_{\widehat{\varphi}}(T)\right)$. In we want to keep truck of the variation of the polynomial $p_{\widehat{\varphi}}(T)$, we need to introduce a relative version of the Grassmannian.

Let $p^{\text {univ }}(T)$ denote the universal polynomial of $\mathbb{A}$; that is, the point corresponding to the identity morphism Id $: \mathbb{A} \rightarrow \mathbb{A}$ and let us think of $\mathcal{O}_{\mathbb{A}}[[z]][T] / p^{\text {univ }}(T)$ as the ring of the universal formal spectral cover. Let us denote:

$$
V:=\mathcal{O}_{\mathbb{A}}((z))[T] / p^{\text {univ }}(T) \quad V^{+}:=\mathcal{O}_{\mathbb{A}}[[z]][T] / p^{\text {univ }}(T)
$$

as sheaves of $\mathcal{O}_{\mathbb{A}}$-modules. 
There exists a notion of commensurability for sub- $\mathcal{O}_{\mathbb{A}}$-modules of $V$ and it satisfies equivalent properties as those of the standard commensurability (see [3]). This notion endows $V$ with a topology. The relative Grassmannian is defined in the same spirit of the usual one (see [36]):

Definition D.13. The relative infinite Grassmannian associated with the couple $\left(V, V^{+}\right)$is the functor that associates the following set with each $\mathbb{A}$-scheme $S$ :

$$
\mathcal{G} \operatorname{r}(V)(S)=\left\{\begin{array}{c}
\text { quasicoherent sub- } \mathcal{O}_{S} \text {-modules } W \subseteq V_{S} \text { such that } V_{S} / W \text { is } \\
\text { flat and for each point in } S \text { there exists an open neighborhood } U \text { and } \\
C \text { commensurable with } V^{+} \text {such that } V_{U} /\left(W_{U}+C_{U}\right)=0 \\
\text { and } W_{U} \cap C_{U} \text { is loc. free of finite type }
\end{array}\right\}
$$

and can be shown that $\mathcal{G} \mathrm{r}(V)$ is representable by an $\mathbb{A}$-scheme (thus, in particular by a $k$-scheme) and moreover it is a trivial fibration over $\mathbb{A}$, that is, a fibration of infinite Grassmannians.

Thus, we can define a Krichever map equivalent to that of equation (D.4.1):

$$
\begin{aligned}
\mathcal{H i g g s}_{X}^{\infty}(k) & \rightarrow \mathcal{G r}\left(\mathcal{O}_{\mathbb{A}}((z))[T] / p^{\text {univ }}(T)\right)(k) \\
(E, \phi, \varphi) & \mapsto \phi_{\varphi} \mathrm{H}^{0}\left(X_{\varphi}-\pi^{-1}(x), E_{\varphi}\right),
\end{aligned}
$$

$\pi: X_{\varphi} \rightarrow X$ being the spectral cover. By Theorem 4.10 it is injective. The image could be characterized in a similar way to that of Theorem D.4.1.

\section{D.5. Moduli of coverings and integrable systems.}

We give in this section some results concerning the moduli space of finite coverings between curves, the moduli space of line bundles over these coverings, and we also discuss the existence of an integrable system that behaves as the Hitchin system.

Let us first note that one has:

Proposition D.14. (Proposition 5.3 Let $\pi: Y \rightarrow X$ be a degree $n$ separable morphism between smooth, projective and integral curves over $k$. Let $x \in X$ be a point such that $\pi^{-1}(x)=\sum_{i=1}^{r} n_{i} y_{i}$ where $y_{i} \in Y, y_{i} \neq y_{j}$ for all $i \neq j$, and $\sum_{i=1}^{r} n_{i}=n$. Then:

- There exists an isomorphism:

$$
z_{x}: \widehat{\mathcal{O}}_{X, x} \simeq k[[z]]
$$

- Let us write $\bar{y}=\pi^{-1}(x)=\sum_{i=1}^{r} n_{i} y_{i}$ where $\sum_{i=1}^{r} n_{i}=n$. For each $y_{i} \in \bar{y}$, there exists a formal parameter on $y_{i}$ :

$$
z_{y_{i}}: \widehat{\mathcal{O}}_{Y, y_{i}} \simeq k\left[\left[z_{i}\right]\right]
$$


- Given $z_{x}$ and given $\left\{z_{y_{i}}\right\}_{i=1}^{r}$ there exist, canonically, irreducible and monic polynomials of degree $n_{i}, p_{i}(T)$, with coefficients in $k[[z]]$, such that the following diagram is a commutative diagram of $k$-algebras:

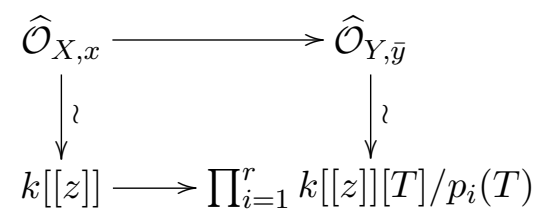

Notice that we can also choose:

$$
p_{i}(T)=T^{n_{i}}-z u_{i}(z, T),
$$

where $u_{i}(z, T) \in k[[z]][T]$ has degree less than $n_{i}$ and $u_{i}(0,0)=1$ (for points with values in a $k$-scheme $S$, one has to endow the category of schemes with the étale topology in order to make this choice).

We denote $\mathcal{C}_{0} v_{\underline{n}}^{\infty}$ the moduli spaces of finite separable coverings between smooth curves with formal extra data. Its rational points are given by the data $\mathcal{Y}=\left(Y, X, \pi, x, \bar{y}, z_{x}, z_{\bar{y}}\right)$ :

1. $\pi: Y \rightarrow X$ is a degree $n$ separable morphism between smooth, integral and projective curves over $k$.

2. $x$ is a point in $X$ and $\bar{y}=\left\{y_{1}, \ldots, y_{r}\right\}$ is a set of points in $y$ verifying

$$
\pi^{-1}(x)=n_{1} y_{1}+\cdots+n_{r} y_{r} .
$$

3. $z_{x}$ is a formal parameter along $x \in X$ :

$$
z_{x}: \widehat{\mathcal{O}}_{X, x} \simeq k[[z]]
$$

4. $z_{\bar{y}}=\left\{z_{y_{i}}\right\}$ is a formal parameter along $\bar{y}$ :

$$
z_{\bar{y}}: \widehat{\mathcal{O}}_{Y, \bar{y}} \simeq k\left[\left[z_{1}\right]\right] \times \cdots \times k\left[\left[z_{r}\right]\right]
$$

in such a way that for each $y_{i} \in \bar{y}, z_{y_{i}}$ is a formal parameter along $y_{i}$ :

$$
z_{y_{i}}: \widehat{\mathcal{O}}_{Y, y_{i}} \simeq k\left[\left[z_{i}\right]\right] \text {. }
$$

5. $\left(Y, X, \pi, x, \bar{y}, z_{x}, z_{\bar{y}}\right)$ and $\left(Y^{\prime}, X^{\prime}, \pi^{\prime}, x^{\prime}, \bar{y}^{\prime}, z_{x^{\prime}}, z_{\bar{y}^{\prime}}\right)$ are said to be equivalent whenever there exists a diagram:

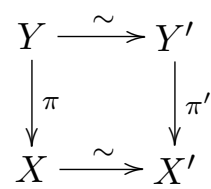

compatible with all data. 
The main difference between this moduli and the Hurwitz moduli studied in [31, Definition 4], is that we are not fixing any compatibility among the formal parameters $z$ and $z_{i}$. In [31] the following compatibility is chosen: $z_{i}^{n_{i}}=z$. That is, the polynomials in their case are fixed and correspond to $p_{i}(T)=T^{n_{i}}-z$.

We can now define a standard Krichever map by:

$$
\begin{aligned}
\mathrm{Kr}: \mathcal{C}_{\operatorname{ov}_{\underline{n}}}^{\infty} & \rightarrow \operatorname{Gr}\left(V_{\underline{n}}\right) \\
\mathcal{Y} & \mapsto z_{\bar{y}} \mathrm{H}^{0}\left(Y-\bar{y}, \mathcal{O}_{Y}\right) .
\end{aligned}
$$

Since we want to keep truck of the polynomials $p_{i}(T)$, to allow them to vary, let us define a slightly different Krichever map. As in the previous section, given a partition $\underline{n}=$ $\left(n_{1}, \ldots, n_{r}\right)$, let us define:

$$
\mathbb{A}_{\underline{n}}:=\mathbb{A}_{n_{1}} \oplus \cdots \oplus \mathbb{A}_{n_{r}},
$$

where $\mathbb{A}_{n_{i}}$ is the $k$-scheme representing the functor:

$$
S \rightsquigarrow \mathcal{O}_{S}[[z]]^{n_{i}}
$$

( $S$ being a $k$-scheme) and whose rational points are thought of as irreducible, monic, separable polynomial of degree $n_{i}$ with coefficients in $k[[z]]$. This implies the existence of a map:

$$
\begin{aligned}
C: \mathcal{C}_{\operatorname{ov}_{\underline{n}}}^{\infty} & \rightarrow \mathbb{A}_{\underline{n}} \\
\mathcal{Y} & \mapsto \underline{p}(T)=\left(p_{1}(T), \ldots, p_{r}(T)\right)
\end{aligned}
$$

used to define the following Krichever map:

$$
\begin{aligned}
& \operatorname{Cov}_{\underline{n}}^{\infty} \rightarrow \operatorname{Gr}\left(V_{\underline{n}}\right) \times \mathbb{A}_{\underline{n}} \\
& \mathcal{Y} \mapsto\left(z_{\bar{y}} \mathrm{H}^{0}\left(Y-\bar{y}, \mathcal{O}_{Y}\right), \underline{p}(T)\right) \text {. }
\end{aligned}
$$

This map allows us to keep truck of the local structure of the covering. Moreover, one can go an step further and define an equivalent Krichever map to this one in terms of the relative Grassmannian.

Let us denote $V_{\underline{n}}^{\text {univ }}$ for the product $\prod_{i=1}^{r} \mathcal{O}_{\mathbb{A}_{n_{i}}}((z))[T] / p_{i}^{\text {univ }}(T)$ with the following $\mathcal{O}_{\mathbb{A}_{\underline{n}}}((z))$-algebra structure:

$$
\begin{aligned}
\mathcal{O}_{\mathbb{A}_{\underline{n}}}((z)) & \rightarrow \mathcal{O}_{\mathbb{A}_{n_{1}}}((z))[T] / p_{1}^{\text {univ }}(T) \times \cdots \times \mathcal{O}_{\mathbb{A}_{n_{r}}}((z))[T] / p_{r}^{\text {univ }}(T) \\
z & \mapsto\left(T^{n_{1}} u_{1}(z, T)^{-1}, \ldots, T^{n_{r}} u_{r}(z, T)^{-1}\right)
\end{aligned}
$$

where $p_{i}^{\text {univ }}(T)$ are the universal polynomials of $\mathbb{A}_{n_{i}}$, and let us write $p_{i}^{\text {univ }}(T)=T^{n_{i}}-$ $z u_{i}(z, T)$, where $u_{i}(z, T) \in \mathcal{O}_{\mathbb{A}_{n_{i}}}[[z]][T]$.

Bearing in mind that:

$$
\operatorname{Gr}\left(V_{\underline{n}}\right) \times \mathbb{A}_{\underline{n}} \simeq \mathcal{G} \mathrm{r}\left(V_{\underline{n}}^{\text {univ }}\right),
$$


we define the following version of the Krichever map:

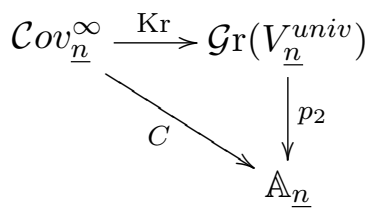

where $C(\mathcal{Y})=\underline{p}(T)$ and $p_{2}$ is the projection on the second factor. Note that the fiber of $C$ at $\underline{p}(T)=\left(T^{n_{1}}-z, \ldots, T^{n_{r}}-z\right)$ is precisely the Hurwitz moduli studied in [31].

Let $\operatorname{Tr}: V_{\underline{n}, p} \rightarrow k((z))$ be the trace map. We have the following characterization of the image of $\mathrm{Kr}$.

Theorem D.5.1. (Theorem 5.11) A rational point $B \in \mathcal{G} \mathrm{r}\left(V_{\underline{n}}^{\text {univ }}\right)$ lies on the image of the Krichever map if and only if $B$ is a regular sub- $k$-algebra of $\bar{V}_{\underline{n}, p}$ and $\operatorname{Tr}(B) \subseteq B$.

The representability of $\mathcal{C}_{\underline{n}}^{\infty}$ a subscheme of the relative Grassmannian is given by the following:

Theorem D.5.2. (Theorem 5.12) $\operatorname{Cov}_{\underline{n}}^{\infty}$ is representable by a locally closed subscheme of $\mathcal{G} \mathrm{r}\left(V_{\underline{n}}^{\text {univ }}\right)$.

Let us now state similar results for the moduli of line bundles over these kind of coverings, $\mathcal{P} i c_{n}^{\infty}$. We will make use of the preceding results and the ones mentioned in Section D.3. Rational points of $\mathcal{P} i c_{\underline{n}}^{\infty}$ consist of tuples $\mathcal{Y}_{L}=\left(Y, X, \pi, x, \bar{y}, z_{x}, z_{\bar{y}}, L, \phi_{\bar{y}}\right)$ where:

1. $\left(Y, X, \pi, x, \bar{y}, z_{x}, z_{\bar{y}}\right)$ is a ration point of $\operatorname{Cov}_{\underline{n}}^{\infty}$

2. $L$ is a line bundle over $Y$.

3. $\phi_{\bar{y}}=\left\{\phi_{y_{i}}\right\}$ is a family of formal trivializations of $L$ along $y_{i}$ :

$$
\phi_{y_{i}}: \widehat{L}_{y_{i}} \simeq \widehat{\mathcal{O}}_{Y, y_{i}} .
$$

We define the Krichever map by:

$$
\begin{aligned}
\mathrm{Kr}: \mathcal{P} i c_{\tilde{\mathcal{Y}}}^{\infty} & \rightarrow \mathcal{G} \mathrm{r}\left(V_{\underline{n}}^{\text {univ }}\right) \\
\mathcal{Y}_{L} & \mapsto\left(z_{\bar{y}} \circ \phi_{\bar{y}}\right) \mathrm{H}^{0}(Y-\bar{y}, L) .
\end{aligned}
$$

We will refer to:

$$
B_{W}:=\left\{f \in V_{\underline{n}, p} \mid f \cdot W \subseteq W\right\}
$$

as the stabilizer algebra of $W$ in $V_{\underline{n}, p}$.

The image of the Krichever map is characterized by the following theorem.

Theorem D.5.3. (Theorem 5.20 A rational point $W \in \mathcal{G} \mathrm{r}\left(V_{\underline{n}}^{\text {univ }}\right)$ lies on the image of the Krichever map if and only if:

- $B_{W} \in \mathcal{G r}\left(V_{\underline{n}}^{u n i v}\right)$ and it is regular. 
- $\operatorname{Tr}\left(B_{W}\right) \subset B_{W}$.

Theorem D.5.4. (Theorem 5.22 $\mathcal{P} i c_{\tilde{\mathcal{Y}}}^{\infty}$ is representable by a subscheme of $\mathcal{G} \mathrm{r}\left(V_{\underline{n}}^{\text {univ }}\right)$.

Remark D.15. The study developed in [1] can be understood in this context as the study of the fibers of $\mathcal{P} i c_{n}^{\infty} \rightarrow \mathbb{A}_{n}$. Their point of view consist of making use of the stabilizer algebra $S_{W}$ of $W$ in $k((z))^{n}$ (which is a non necessary commutative sub-k-algebra of $\left.\operatorname{End}_{k((z))} k((z))^{n}\right)$ and to cut this algebra with a Heisenberg algebra in order to obtain a commutative ring. This ring allow them to recover the curve upstairs. With our techniques, their situation can be recover as follows. The image of the natural inclusion:

$$
V_{\underline{n}, p} \hookrightarrow \operatorname{End}_{k((z))} k((z))^{n}
$$

is precisely the Lie algebra of the Heisenberg group (see Section D.1), and one can check that:

$$
S_{W} \cap V_{\underline{n}, p}=B_{W} \cdot
$$

Remark D.16. The fibers of $\mathcal{P} i c_{\underline{n}}^{\infty} \rightarrow \mathbb{A}_{\underline{n}}$ are also studied in [24]. They establish a categorical correspondence among the geometric data and the algebraic data, which in their case consist of tuples $\left(A_{0}, A_{n}, W\right)$, where $A_{0}$ is related with the curve downstairs, $A_{n}$ with the curve upstairs and $W$ with the bundle. From our point of view, the data $\left(A_{0}, A_{n}, W\right)$ is characterized only by $W$ (the curve upstairs is recovered with $B_{W}$, the one downstairs with $\operatorname{Tr}\left(B_{W}\right)$ and the bundle with $W$ ).

Let us proceed to look for an integrable system with analogue properties to those of the Hitchin system. Let us fix the curve $X$ (and the formal data associated to it) and let us denote $\mathcal{P} i c_{n, X}^{\infty}$ for the moduli space of line bundles over finite coverings of $X$ (with formal extra data). Then we have a forgetful map:

$$
\mathcal{P} i c_{\underline{n}}^{\infty} \rightarrow \mathcal{P} i c_{\underline{n}, X}^{\infty}
$$

and we can define a map:

$$
J: \mathcal{P}_{\underline{n}, X}^{\infty} \rightarrow \mathbb{A}_{\underline{n}} .
$$

At a point $p(T)$ of $\mathbb{A}_{\underline{n}}$, the fiber of $J$ is a family of Jacobians with formal extra data (in fact, a finite family if we fix the genus of $X$ ). As opposite as in the case of the Hitchin system, where the fiber at a generic point of the Hitchin map is one Jacobian (the Jacobian of the spectral curve), we have a family of Jacobians.

In order to state an analogy between the system $J$ with the formal analogue $\mathcal{H i g g s}_{X}^{\infty} \rightarrow$ $\mathbb{A}$ of the Hitchin system, let us define the following Ind-scheme:

$$
\mathbb{O}_{x}:=\mathcal{O}_{X, x} \times \cdots \times \mathcal{O}_{X, x}
$$

(notice that $\mathcal{O}_{X, x}=\lim _{D \geq 0, x \notin D} \mathrm{H}^{0}\left(X, \mathcal{O}_{X}(D)\right)$, this is the reason why it is not a scheme but an Ind-scheme). We will think of its points $\left(o_{1}, \ldots, o_{n}\right)$ as polynomials:

$$
p(T)=T^{n}+o_{1} T^{n-1}+\cdots+o_{n}
$$


and restrict ourselves to the open subset on which $\mathcal{O}_{X, x}[T] / p(T)$ is an integral, regular, separable $\mathcal{O}_{X, x}$-algebra with partition data $\underline{n}$. Note also that one can pass from $\mathbb{O}_{x}$ to $\mathbb{A}_{\underline{n}}$ by taking the completion at $x$. Now, the key point is the following proposition.

Proposition D.17. Let $\left(a_{1}, \ldots, a_{n}\right)$ be a point in $\mathbb{A}$. If $\left(a_{1}, \ldots, a_{n}\right) \in \mathbb{O}_{x}$ then, there exists a unique degree $n$ covering, $Y \rightarrow X$, and the fiber of $\mathcal{P} i c_{\underline{n}}^{\infty} \rightarrow \mathbb{A}_{\underline{n}}$ at $\left(a_{1}, \ldots, a_{n}\right) \in \mathbb{O}_{x}$ is the Jacobian $\mathcal{J}^{\infty}(Y)$ of line bundles with formal trivialization data.

Now it is clear that the fibration:

$$
\mathcal{P} i c_{\underline{n}, X}^{\infty} \mathbb{O}_{x} \rightarrow \mathbb{O}_{x}
$$

is an algebraically integrable system in the classical sense and, moreover, we have the following diagram:

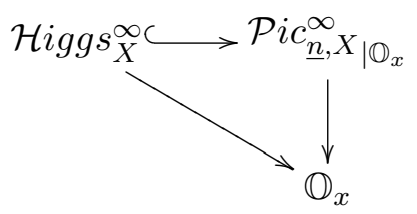

which relates Jacobians of curves with Jacobians of spectral curves.

To finish with, let us study the relationship between fiber bundles over a curve $X$ and line bundles over a finite covering of $X$ (see [7, 21] for the classical results). Let us also say a few words about what is going on for the case on which one adds the formal data.

Let us write $\mathcal{U}_{X}$ for the stack of rank $n$ vector bundles over a smooth, integral, projective curve $X$ over $k$. Denote by $\mathcal{P} i c_{\underline{n}, X}$ the stack of couples $(\pi: Y \rightarrow X, L)$.

Theorem D.5.5. (Theorem 5.31) The natural morphism:

$$
\begin{aligned}
\mathcal{P}_{i c_{\underline{n}, X}} & \rightarrow \mathcal{U}_{X} \\
(Y, L) & \mapsto \pi_{*} L
\end{aligned}
$$

is surjective.

In fact, the proof shows that the following map is surjective:

$$
\overline{\mathcal{H i g g s}}_{X} \rightarrow \mathcal{U}_{X}
$$

where $\overline{\mathcal{H i g g s}}_{X}$ denotes the stack of Higgs pairs over $X$ where the line bundle $N$ of the Higgs field $E \rightarrow E \otimes N$ (twisted endomorphism in the sense of [7]) is allow to vary. It is clear that:

$$
\overline{\mathcal{H i g g s}}_{X} \hookrightarrow \mathcal{P} i c_{\underline{n}, X}
$$

The same result is also true if we add formal trivialization data on $\mathcal{P} i c_{\underline{n}, X}$. The map:

$$
\mathcal{P} i c_{\underline{n}, X}^{\infty} \rightarrow \mathcal{P} i c_{\underline{n}, X} \rightarrow \mathcal{U}_{X}
$$

is surjective. 
Nevertheless, if one adds a formal trivialization on the bundle $E$ around $x$, then the map:

$$
\begin{aligned}
\mathcal{P} i c_{\underline{n}, X}^{\infty} & \rightarrow \mathcal{U}_{X}^{\infty} \times \mathbb{A}_{\underline{n}} \\
\left(Y, \pi, z_{\bar{y}}, L, \phi_{\bar{y}}\right) & \mapsto\left(\left(\pi_{*} L, \pi_{*} \phi_{\bar{y}}\right), p(T)\right)
\end{aligned}
$$

is injective. In particular, also the following map is injective:

$$
\mathcal{P} i c_{\underline{n}, X \mid \mathbb{O}_{x}}^{\infty} \rightarrow \mathcal{U}_{X}^{\infty} \times \mathbb{O}_{x}
$$

The reason why it can not be surjective is encoded in the formal trivialization data. This fact can be easily explained in terms of group schemes as follows.

Consider the diagram:

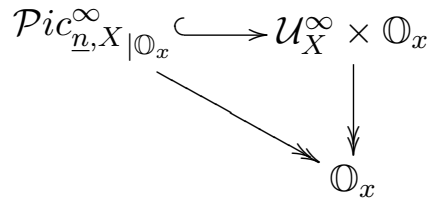

On one side, for each $p(T) \in \mathbb{O}$, the group that acts on $\mathcal{U}_{X}^{\infty}$ changing the formal trivialization of the bundle $E$ at $x$ is $\mathrm{Gl}_{k[[z]]}\left(k[[z]]^{n}\right.$ ) (see Observación 6.9), where we are thinking of $\mathcal{U}_{X}^{\infty}$ as the fiber at $p(T)$ of $\mathcal{U}_{X}^{\infty} \times \mathbb{O}_{x} \rightarrow \mathbb{O}_{x}$.

On the other side, let $\mathcal{J}^{\infty}\left(Y_{p}\right)$ the fiber of $\mathcal{P} i c_{\underline{n}, X}^{\infty} \mathbb{O}_{x} \rightarrow \mathbb{O}_{x}$ at $p(T) \in \mathbb{O}$. The group scheme acting on $\mathcal{J}^{\infty}\left(Y_{p}\right)$ changing the formal trivialization of the line bundles is the group scheme of invertible elements $\Gamma_{V^{+}}$, where $V^{+}=k[[z]][T] / p(T)$.

The image of $\Gamma_{V^{+}}$under the embedding:

$$
\underline{V}^{+, *} \hookrightarrow \mathrm{Gl}_{k((z))}\left(k[[z]]^{n}\right)
$$

(where we write $\underline{V}^{+, *}$ for the functor of invertible elements of $V^{+}$) is the Heisenberg group Heis $_{V^{+}}$(see Section 1.3 or Section D.1 of this appendix). If $\pi: Y \rightarrow X$ is a covering as before, and $E$ is a vector bundle on $X$ such that $E \simeq \pi_{*} L$ (where $L$ is a line bundle on $Y$ ), the Heisenberg group is exactly the group acting on the formal trivialization (at $x$ ) of bundles $E$ which are a direct image of formal trivializations of line bundles $L$. Now it is clear the there exists formal trivializations of $E$ at $x$ which do not come from formal trivializations of $L$ at $\pi^{-1}(x)$.

\section{D.6. Tangent spaces and local generators.}

This section is devoted to compute the tangent spaces of the moduli spaces treated along the work, and to show that the group schemes studied in section D.1, play the role of local generators for these moduli spaces.

Let us first recall some known results on this subject. If we denote by $\mathcal{M}$ the moduli space of smooth curves, it is known that its tangent space at a point $X$ is the first cohomology group 
$\mathrm{H}^{1}\left(X, \operatorname{Der}_{k}\left(\mathcal{O}_{X}, \mathcal{O}_{X}\right)\right)$. If we add a formal parameter $z$ at a marked point $x \in X$ and denote the corresponding moduli space by $\mathcal{M}^{\infty}$, then we have ([33]):

$$
\mathrm{T}_{(X, x, z)} \mathcal{M}^{\infty} \simeq \underset{m}{\lim _{m}} \mathrm{H}^{1}\left(X, \operatorname{Der}_{k}\left(\mathcal{O}_{X}, \mathcal{O}_{X}(-m x)\right)\right)
$$

If we think of $\mathcal{M}^{\infty}$ as a subscheme of the infinite Grassmannian $\operatorname{Gr}(k((z)))$ (whose tangent space at a point $A$ is isomorphic to $\left.\operatorname{Hom}_{k}(A, k((z)) / A)\right)$ then ([32, Prop.4.2]):

$$
\mathrm{T}_{(X, x, z)} \mathcal{M}^{\infty} \simeq \operatorname{Der}_{k}(A, k((z)) / A) .
$$

Theorem 4.11 of [34] shows that the group $\mathrm{G}$ of $k$-algebra automorphisms of $k((z))$ locally generates $\mathcal{M}^{\infty}$, in the sense that it acts on $\mathcal{M}^{\infty}$ and the orbit map is surjective at the level of tangent spaces.

It is also know that the tangent space at a rational point $E$ of the moduli $\mathcal{U}_{X}$ of vector bundles over a smooth curve $X$ is isomorphic to $\mathrm{H}^{1}\left(X, \mathcal{E} \operatorname{nd}_{X} E\right)$. In this case, when adding formal trivialization data we get:

$$
\mathrm{T}_{(E, \phi)} \mathcal{U}_{X}^{\infty} \simeq{\underset{m}{\lim }}_{\mathrm{H}^{1}}\left(X, \mathcal{E} \operatorname{nd}_{X} E(-m x)\right) .
$$

Thinking of $\mathcal{U}_{X}^{\infty}$ as a subscheme of $\operatorname{Gr}\left(k((z))^{n}\right)$, one has:

$$
\mathrm{T}_{(E, \phi)} \mathcal{U}_{X}^{\infty} \simeq \operatorname{Hom}_{A}(W, V / W)
$$

(see Proposition 6.11). Theorem 6.10 states that the action of $\mathrm{Gl}_{k((z))}(V)$ on $\mathrm{Gr}(V)$ induces an action on $\mathcal{U}_{X}^{\infty}$ which is locally transitive, that is, $\mathrm{Gl}_{k((z))}(V)$ is the local generator for $\mathcal{U}_{X}^{\infty}$.

If one wants to allow both data to vary, curve $X$ and vector bundle $E$, is not hard to see that (see Theorem 6.12):

$$
\mathrm{T}_{(X, E)} \mathcal{U} \simeq \mathrm{H}^{1}\left(X, \mathcal{D}_{X / k}^{1}(E, E)\right),
$$

where $\mathcal{U}$ denotes the moduli of curves and vector bundles and we write $\mathcal{D}_{X / k}^{1}(E, E)$ for the scalar differential operators of order one. Recall that there exists an exact sequence:

$$
0 \rightarrow \mathcal{E}_{\operatorname{nd}_{X}} E \rightarrow \mathcal{D}_{X / k}^{1}(E, E) \rightarrow \operatorname{Der}_{k}\left(\mathcal{O}_{X}, \mathcal{O}_{X}\right) \rightarrow 0
$$

(See also [48] and [4] for another aproach in the case of line bundles). Denoting by $\mathcal{U}^{\infty}$ for the moduli space of curves and vector bundles with formal trivialization data, we show that, at a rational point $\mathcal{E}=(X, x, z, E, \phi)$, the tangent space is:

$$
\mathrm{T}_{\mathcal{E}} \mathcal{U}^{\infty} \simeq \underset{m}{\lim _{m}} \mathrm{H}^{1}\left(X, \mathcal{D}_{X / k}^{1}(E, E(-m x))\right)
$$

In terms of the infinite Grassmannian we have (see Proposition 6.17):

$$
\mathrm{T}_{\mathcal{E}} \mathcal{U}^{\infty} \simeq \mathcal{D}_{A_{W} / k}^{1}(W, V / W),
$$


(where $A_{W}$ denotes the stabilizer algebra of $W$ in $k((z))$ ) and the exact sequence:

$$
0 \rightarrow \operatorname{Hom}_{A}(W, V / W) \rightarrow \mathcal{D}_{A_{W} / k}^{1}(W, V / W) \rightarrow \operatorname{Der}_{k}\left(A_{W}, k((z)) / A_{W}\right) \rightarrow 0
$$

is the analogue, in terms of the Grassmannian, to the exact sequence:

$$
0 \rightarrow \mathrm{T}_{(E, \phi)} \mathcal{U}_{X}^{\infty} \rightarrow \mathrm{T}_{\mathcal{E}} \mathcal{U}^{\infty} \rightarrow \mathrm{T}_{(X, x, z)} \mathcal{M}^{\infty} \rightarrow 0
$$

The group that locally generates this moduli is the group $\mathrm{SGl}_{k((z))}(V)$ of semilinear automorphisms of $V=k((z))^{n}$ (see Theorem 6.14) and we can deduce a diagram:

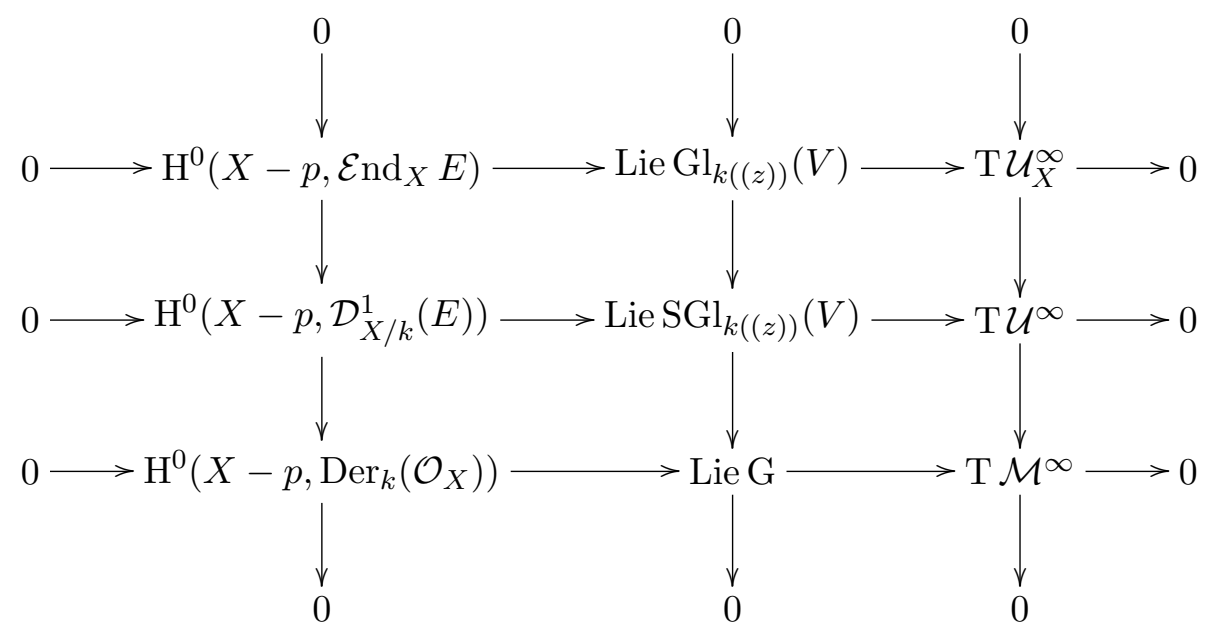

In particular, for the case of line bundles we recover some of the results given in [4] and [32].

Let us now denote $\mathcal{C}$ ov for the moduli space of finite, separable coverings $\pi: Y \rightarrow X$ between integral, smooth and projective curves. We define the $\pi$-equivariant derivations, $\operatorname{Der}_{k}\left(\mathcal{O}_{Y}, \mathcal{O}_{Y}\right)^{\pi}$, as those derivations from $\mathcal{O}_{Y}$ to $\mathcal{O}_{Y}$ compatible with the map $\pi: \mathcal{O}_{X} \rightarrow$ $\pi_{*} \mathcal{O}_{Y}$, that is, derivations $\theta^{Y} \in \operatorname{Der}_{k}\left(\mathcal{O}_{Y}, \mathcal{O}_{Y}\right)$ for which there exists $\theta^{X} \in \operatorname{Der}_{k}\left(\mathcal{O}_{X}, \mathcal{O}_{X}\right)$ making the following diagram commutative:

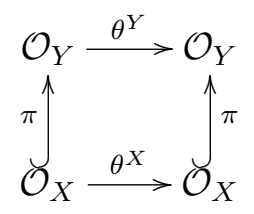

In the same way, we can define Tr-equivariant derivations $\operatorname{Der}_{k}\left(\mathcal{O}_{Y}, \mathcal{O}_{Y}\right)^{\operatorname{Tr}}$, where:

$$
\operatorname{Tr}: \pi_{*} \mathcal{O}_{Y} \rightarrow \mathcal{O}_{X}
$$

is the trace map of $\mathcal{O}_{Y}$ as a finite $\mathcal{O}_{X}$-algebra. One can show that (see Proposition 6.21):

$$
\operatorname{Der}_{k}\left(\mathcal{O}_{Y}, \mathcal{O}_{Y}\right)^{\pi} \simeq \operatorname{Der}_{k}\left(\mathcal{O}_{Y}, \mathcal{O}_{Y}\right)^{\operatorname{Tr}}
$$


Let $(X, Y, \pi)$ be a rational point of $\mathcal{C} o v$, the main result for this space is the following (see Proposition 6.22):

$$
\mathrm{T}_{(X, Y, \pi)} \mathcal{C} \text { ov } \simeq \mathrm{H}^{1}\left(X, \operatorname{Der}_{k}\left(\mathcal{O}_{Y}, \mathcal{O}_{Y}\right)^{\pi}\right) \oplus \mathrm{H}^{0}\left(X, \operatorname{Der}_{k}\left(\mathcal{O}_{X}, \mathcal{O}_{Y}\right)\right)
$$

Moreover, when the genus $g_{X}$ of $X$ is bigger than 1, the space:

$$
\mathrm{H}^{0}\left(X, \operatorname{Der}_{k}\left(\mathcal{O}_{X}, \mathcal{O}_{Y}\right)\right)
$$

(which measures the first order infinitesimal deformations of the map $\pi$ ) is zero, so the tangent space is only $\mathrm{H}^{1}\left(X, \operatorname{Der}_{k}\left(\mathcal{O}_{Y}, \mathcal{O}_{Y}\right)^{\pi}\right)$. We also include similar results for the case on which the base curve $X$ is fixed.

Let us write $\mathcal{C}_{0 v}^{\infty}$ for the moduli of pointed coverings with formal extra data (see section D.5 and let $\mathcal{Y}$ be a rational point. For $g_{X}>1$ one has the following isomorphisms of $k$ vector spaces:

$$
\mathrm{T}_{\mathcal{Y}} \mathcal{C o v}_{\underline{\underline{n}}}^{\infty} \simeq \underset{m}{\lim _{m}} \mathrm{H}^{1}\left(X, \operatorname{Der}_{k}\left(\mathcal{O}_{Y}, \mathcal{O}_{Y}(-m \bar{y})\right)^{\pi}\right)
$$

It can be shown that the group $\mathrm{G}_{\underline{n}}$ is the local generator for this moduli space (see Theorem 6.29).

In order to reformulate the tangent space in terms of the relative Grassmannian $\mathcal{G} r\left(V_{\underline{n}}^{\text {univ }}\right)$, let us recall that we have an isomorphism:

$$
\mathcal{G} \mathrm{r}\left(V_{\underline{n}}^{\text {univ }}\right) \simeq \operatorname{Gr}(V) \times \mathbb{A}_{\underline{n}}
$$

so that, any rational point $B$ of $\mathcal{G} r\left(V_{\underline{n}}^{\text {univ }}\right)$ can be thought of as a couple $\left(B_{p}, p(T)\right)$ in $\operatorname{Gr}\left(V_{\underline{n}, p}\right) \times \mathbb{A}_{\underline{n}}$. Thus, the tangent space at $B$ of $\mathcal{G} \mathrm{r}\left(V_{\underline{n}}^{\text {univ }}\right)$ is given by Theorem 6.34.

$$
\mathrm{T}_{B} \mathcal{G} \mathrm{r}\left(V_{\underline{n}}^{\text {univ }}\right) \simeq \operatorname{Hom}_{k}\left(B_{p}, V_{\underline{n}, p} / B_{p}\right) \oplus \mathrm{T}_{p(T)} \mathbb{A}_{\underline{n}} .
$$

Remember also that we have a map $C: \mathcal{C}_{0} v_{\underline{n}}^{\infty} \rightarrow \mathbb{A}_{\underline{n}}$ sending $\mathcal{Y}$ to $p(T)$ (see section D.5p. Then, the tangent space of $\mathcal{C}_{0} v_{\underline{n}}^{\infty}$ can be described in terms of the tangent space of $\mathcal{G} \mathrm{r}\left(V_{\underline{n}}^{\text {univ }}\right)$ by the isomorphism:

$$
\mathrm{T}_{\mathcal{Y}} \mathcal{C}_{o v}^{\infty} \underset{\underline{n}}{\simeq} \operatorname{Der}_{k}\left(B_{p}, V_{\underline{n}, p} / B_{p}\right)^{\operatorname{Tr}} \oplus \mathrm{T}_{p(T)} \operatorname{Im}(C) .
$$

For the case of $\mathcal{P} i c_{\underline{n}}$, the moduli space of line bundles over coverings as before (with $g_{X}>1$ ), we only have to glue the results for $\mathcal{C}$ ov and the ones for $\mathcal{U}$ in order obtain the following isomorphism of $k$-vector espaces (see Proposition 6.36):

$$
\mathrm{T}_{(X, Y, \pi, L)} \mathcal{P} i c_{\underline{n}} \simeq \mathrm{H}^{1}\left(Y, \mathcal{D}_{Y / k}^{1}(L, L)^{\pi}\right) .
$$

Adding formal trivialization data changes the result as follows:

$$
\mathrm{T}_{P} \mathcal{P} i c_{\underline{n}}^{\infty} \simeq \underset{m}{\lim _{m}} \mathrm{H}^{1}\left(Y, \mathcal{D}_{Y / k}^{1}(L, L(-m \bar{y}))^{\pi}\right) .
$$


In this case the group that plays the role of local generator is $\operatorname{SHeis}_{k} \frac{V_{n}}{((z))}$ (see Theorem 6.39), and in terms of the tangent space of the relative Grassmannian, the tangent space of $\mathcal{P} i c_{\underline{n}}$ is given by Theorem 6.41

$$
\mathrm{T}_{\mathcal{Y}_{L}} \mathcal{P} i c_{\underline{n}}^{\infty} \simeq \mathcal{D}_{B_{W_{p}} / k}^{1}\left(W_{p}, V_{\underline{n}, p} / W_{p}\right)^{\operatorname{Tr}} \oplus \mathrm{T}_{p(T)} \operatorname{Im}(D),
$$

where $D: \mathcal{P} i c_{\underline{n}}^{\infty} \rightarrow \mathbb{A}_{\underline{n}}$ is the map that sends a point $\mathcal{Y}_{L}$ of $\mathcal{P} i c_{\underline{n}}^{\infty}$ to the polynomial $p(T)$ which defines the local structure of the covering.

Let us finish saying few words about the tangent space of the moduli space of Higgs pairs $\mathcal{H i g g s}_{X}$. Let $(E, \varphi)$ be a rational point, it is know that:

$$
\mathrm{T}_{(E, \varphi)} \mathcal{H i g g s}_{X} \simeq \mathbb{H}^{1}([, \varphi])
$$

where $[, \varphi]: \mathcal{E} \operatorname{nd}_{X} E \rightarrow \mathcal{E} \operatorname{nd}_{X} E \otimes \omega_{X}$ is the complex defined by:

$$
[, \varphi](\eta):=[\eta, \varphi]=(\eta \otimes 1) \circ \varphi-\varphi \circ \eta .
$$

Nevertheless, we are going to use another interpretation for this tangent space, the one given in [29]:

$$
\mathrm{T}_{(E, \varphi)} \mathcal{H i g g s}_{X} \simeq \mathrm{H}^{1}\left(X_{\varphi}, \mathcal{O}_{X_{\varphi}}\right) \oplus \mathrm{T}_{\operatorname{ch}(\varphi)} \mathcal{H}
$$

where $X_{\varphi}$ refers to the spectral curve associated to $(E, \varphi), \mathcal{H}=\oplus_{i=1}^{n} \mathrm{H}^{0}\left(X, \omega_{X}^{\otimes i}\right)$ is the Hitchin base and $\operatorname{ch}(\varphi)$ are the characteristic coefficients of the Higgs field $\varphi$. Moreover, since $\mathcal{H}$ is an affine space one has $\mathrm{T}_{\operatorname{ch}(\varphi)} \mathcal{H} \simeq \mathcal{H}$.

Let $\mathcal{H}_{\text {iggs }}^{\infty}{ }_{X}^{\infty}$ be the moduli space of Higgs pairs with formal extra data (see section D.4) and let $\mathcal{E}$ be a rational point. Bearing in mind the result above, and the fact that we have a formal analogue $\mathcal{H}_{\infty}: \mathcal{H} i g g s_{X}^{\infty} \rightarrow \mathbb{A}$ to the Hitchin map, it is not hard to figure out that:

$$
\mathrm{T}_{\mathcal{E}} \mathcal{H} i g g s_{X}^{\infty} \simeq{\underset{m}{\lim }}^{1} \mathrm{H}^{1}\left(X_{\varphi}, \mathcal{O}_{X_{\varphi}}(-m)\right) \oplus \mathcal{H}
$$

(note that the image of $\mathcal{H}_{\infty}$ is $\mathcal{H}$ ).

In terms of the tangent space of the relative Grassmannian this results takes the form:

$$
\mathrm{T}_{\mathcal{E}} \mathcal{H i g g s}_{X}^{\infty} \simeq \operatorname{Hom}_{B_{W_{p}}}\left(W_{p}, V_{p} / W_{p}\right) \oplus \mathcal{H}
$$

\section{D.7. Equations of the moduli of Higgs pairs.}

This section provides concrete equations describing $\mathcal{H} i g g s_{X}^{\infty}$ in terms of Baker-Akhiezer functions and, for the subscheme of $\mathcal{H} i g g s_{X}^{\infty}$ on which the spectral cover is totally ramified, the equations are explicitly computed in terms of the characteristic coefficients of the Higgs field.

Let $p(T) \in \mathbb{A}$ be a rational point and let us denote:

$$
V_{p}=k((z))[T] / p(T) \quad V_{p}^{+}=k[[z]][T] / p(T) .
$$


From now on, let us assume that $V_{p}$ is a separable $k((z))$-algebra with the following decomposition:

$$
V_{p} \simeq V_{1} \times \cdots \times V_{r},
$$

where $V_{i}=k((z))\left[T_{i}\right] / T_{i}^{n_{i}}-z u_{i}\left(T_{i}\right), n_{1}+\cdots+n_{r}=n$ and $u_{i}\left(T_{i}\right)$ are invertible in $k\left[\left[T_{i}\right]\right]$. In addition, $k\left(\left(T_{i}\right)\right)$ can be endowed with a $k((z))$-álgebra structure by sending $z$ to $T_{i}^{n_{i}} u_{i}\left(T_{i}\right)^{-1}$. Consequently one obtains an isomorphism:

$$
k\left(\left(T_{i}\right)\right) \simeq V_{i}=k((z))\left[T_{i}\right] / T_{i}^{n}-z u\left(T_{i}\right)
$$

This assumption is motivated by the following geometric fact:

Proposition D.18. (Proposition 7.1) Let us consider a rational point $(E, \varphi, \phi)$ in $\mathcal{H}_{\text {iggs }}^{\infty}{ }_{X}^{\infty}$, and let $\pi: X_{\varphi} \rightarrow X$ be the associated spectral cover. Let us assume that $\pi^{-1}(x)=\sum_{i=1}^{r} n_{i} y_{i}$, where $\sum_{i_{1}}^{r} n_{i}=n$ and $y_{i} \neq y_{j}$ for all $i \neq j$. We then have:

$$
\widehat{\mathcal{O}}_{X_{\varphi}, \pi^{-1}(x)} \simeq\left(k[[z]]\left[T_{1}\right] / T_{1}^{n_{1}}-z u_{1}\left(T_{1}\right)\right) \times \cdots \times\left(k[[z]]\left[T_{r}\right] / T_{r}^{n_{r}}-z u_{r}\left(T_{r}\right)\right),
$$

where $u_{i}\left(T_{i}\right) \in k[[z]]\left[T_{i}\right]$.

Let us consider the fibration of infinite Grassmannians:

$$
\operatorname{Gr}\left(k((z))^{n}\right) \times \mathbb{A} \rightarrow \mathbb{A}
$$

and let $p(T)$ be a rational point of $\mathbb{A}$. The fiber at $p(T) \in \mathbb{A}$ is $\operatorname{Gr}\left(V_{p}\right)$.

Let us now consider the natural action of $\Gamma_{V_{p}}$ in $\operatorname{Gr}\left(V_{p}\right)$ :

$$
\begin{aligned}
\mu: \Gamma_{V_{p}} \times \operatorname{Gr}\left(V_{p}\right) & \rightarrow \operatorname{Gr}\left(V_{p}\right) \\
\left(g_{\bullet}, W\right) & \mapsto g_{\bullet} \cdot W,
\end{aligned}
$$

(where $\Gamma_{V_{p}}$ is the connected component at the origin of the formal scheme of invertible elements in $V_{p}$, see Section D.1 and let us define the Poincaré sheaf on $\Gamma_{V_{p}} \times \operatorname{Gr}\left(V_{p}\right)$ by:

$$
\mathcal{P}:=\mu^{*} \operatorname{Det}_{V_{p}}^{*}
$$

For each rational point $W \in \operatorname{Gr}\left(V_{p}\right)$, one has an invertible sheaf on $\Gamma_{V_{p}}$ :

$$
\widetilde{\mathcal{L}}_{\tau}(W):=\mathcal{P}_{\mid \Gamma_{V_{p}} \times\{W\}}
$$

and a natural homomorphism:

$$
\mathrm{H}^{0}\left(\Gamma_{V_{p}} \times \operatorname{Gr}\left(V_{p}\right), \mathcal{P}\right) \rightarrow \mathrm{H}^{0}\left(\Gamma_{V_{p}} \times\{W\}, \widetilde{\mathcal{L}}_{\tau}(W)\right) .
$$

Definition D.19. We use the term $\tau$-section of a point $W$, $\tilde{\tau}_{W}$, to refer to the image of $\mu^{*} \Omega_{+}$ under the morphism $\left(\right.$ D.7.1), where $\Omega_{+}$is the canonical global section of $\operatorname{Det}_{V_{p}}^{*}$ of Definition 3.14 in [3]. 
Note that $\tilde{\tau}_{W}$ is not a true function over $\Gamma_{V_{p}} \times\{W\}$, because $\widetilde{\mathcal{L}}_{\tau}(W)$ is in general nontrivial. The algebraic analogue of the tau function defined by Sato-Sato ([40]) and SegalWilson ([42]) is obtained by restricting $\widetilde{\mathcal{L}}_{\tau}(W)$ to the formal subgroup $\Gamma_{V_{p}}^{-} \simeq \mathcal{J}\left(\widehat{X}_{V}\right) \subset$ $\Gamma_{V_{p}}$.

Let us define $\mathcal{L}_{\tau}(W):=\widetilde{\mathcal{L}}_{\tau}(W)_{\mid \Gamma_{V_{p}}^{-} \times\{W\}}$, which is trivial over $\Gamma_{V_{p}}^{-} \times\{W\}$. Indeed a trivialization of $\mathcal{L}_{\tau}(W)$ can be given by the global section:

$$
\sigma_{0}\left(g_{\bullet}\right):=g_{\bullet} \cdot \rho_{W},
$$

where $\rho_{W}$ is a non-zero element in the fiber of $\mathcal{L}_{\tau}(W)$ over the point $(1, W) \in \Gamma_{V_{p}} \times W$. The $\tau$-function is defined as the trivialization of the restriction of $\widetilde{\tau}_{W}$ to $\Gamma_{V_{p}}$.

Definition D.20. The $\tau$-function of the point $W, \tau_{W}$, is the function:

$$
\tau_{W} \in \mathcal{O}_{\Gamma_{V_{p}}^{-}} \simeq \mathcal{O}_{\mathcal{J}\left(\widehat{X}_{V}\right)} \simeq k\left\{\left\{t_{1}^{(1)}, \ldots\right\}\right\} \hat{\otimes} \cdots \hat{\otimes} k\left\{\left\{t_{1}^{(r)}, \ldots\right\}\right\}
$$

defined by:

$$
\tau_{W}\left(g_{\bullet}\right)=\frac{\tilde{\tau}_{W}\left(g_{\bullet}\right)}{\sigma_{0}\left(g_{\bullet}\right)}=\frac{\mu^{*} \Omega_{+}\left(g_{\bullet}\right)}{\sigma_{0}\left(g_{\bullet}\right)}=\frac{\Omega_{+}\left(g_{\bullet} \cdot W\right)}{g_{\bullet} \cdot \rho_{W}}
$$

for $g_{\bullet}=\prod_{i=1}^{r} \exp \left(\sum_{j \geq 1} \frac{t_{j}^{(i)}}{T_{i}^{j}}\right) \in \mathcal{J}\left(\widehat{X}_{V}\right) \simeq \Gamma_{V_{p}}^{-}$.

Remark D.21. In particular, over the formal base curve $\widehat{X}=\operatorname{Spf} k[[z]]$ the $\tau$ function of a point $\Omega \in \operatorname{Gr}(k((z)))$ is the function in $\mathcal{J}(\widehat{X}) \simeq \Gamma^{-} \simeq \operatorname{Spf} k\left\{\left\{t_{1}, \ldots\right\}\right\}$ defined by ([3]):

$$
\tau_{\Omega}(g)=\frac{\tilde{\tau}_{\Omega}(g)}{\sigma_{0}(g)}=\frac{\mu^{*} \Omega_{+}(g)}{\sigma_{0}(g)}=\frac{\Omega_{+}(g W)}{g \rho_{W}},
$$

where $g=\exp \left(\sum_{j \geq 1} \frac{t_{j}}{z^{j}}\right) \in \mathcal{J}(\widehat{X}) \simeq \Gamma^{-}$.

Let us now consider the composition:

$$
\tilde{\beta}: \widehat{X}_{V} \times \Gamma_{V_{p}} \times \operatorname{Gr}\left(V_{p}\right) \stackrel{\phi_{1} \times \mathrm{Id}}{\rightarrow} \Gamma_{V_{p}} \times \Gamma_{V_{p}} \times \operatorname{Gr}\left(V_{p}\right) \stackrel{m \times \operatorname{Id}}{\rightarrow} \Gamma_{V_{p}} \times \operatorname{Gr}\left(V_{p}\right)
$$

where $\phi_{1}: \widehat{X}_{V} \rightarrow \Gamma_{V_{p}}$ is the Abel morphism of degree one (with values in $\Gamma_{V_{p}}^{-} \simeq \mathcal{J}\left(\widehat{X}_{V}\right) \subset$ $\Gamma_{V_{p}}$ ) and $m$ is the group law in $\Gamma_{V_{p}}$. Given a point $W \in \operatorname{Gr}\left(V_{p}\right)$, let us denote by $\tilde{\beta}_{W}$ the restriction of $\tilde{\beta}$ to $\widehat{X}_{V} \times \Gamma_{V_{p}} \times\{W\}$.

Definition D.22. The BA-section of $W \in \operatorname{Gr}\left(V_{p}\right)$ is $\tilde{\psi}_{W}:=v^{-1} \cdot \beta_{W}^{*}\left(\tilde{\tau}_{W}\right)$, where:

$$
\beta_{W}^{*}: \mathrm{H}^{0}\left(\Gamma_{V_{p}} \times\{W\}, \widetilde{\mathcal{L}}_{\tau}(W)\right) \rightarrow \mathrm{H}^{0}\left(\widetilde{X}_{V} \times \Gamma_{V_{p}} \times\{W\}, \tilde{\beta}_{W}^{*} \tilde{\mathcal{L}}_{\tau}(W)\right)
$$

is the morphism induced by $\tilde{\beta}_{W}^{*}$. That is, let us choose it in such a way that:

$$
{\widetilde{\psi_{W}}}_{\mid x_{0}^{(i)} \times \Gamma_{V_{p}}}=v_{i}^{-1}
$$


where $x_{0}^{(i)}$ is the origin of $\widehat{X}_{V_{i}}$ and $v^{-1}$ is $v_{i}^{-1}$ in the component $\widehat{X}_{V_{i}}$.

The BA-function of a point $W \in \operatorname{Gr}\left(V_{p}\right)$ is defined by the following formula:

$$
\psi_{W}\left(T_{\bullet}, g_{\bullet}\right)=v^{-1} \frac{\tau_{W}\left(g_{\bullet} \cdot \phi_{1}\left(T_{\bullet}\right)\right)}{\tau_{W}\left(g_{\bullet}\right)},
$$

where $T_{\bullet}=\left(T_{1}, \ldots, T_{r}\right)$ and $g_{\bullet} \in \Gamma_{V_{p}}^{-}$.

In order to give an explicit expression of this function, we identify the formal Jacobian $\mathcal{J}\left(\widehat{X}_{V}\right)$ with $\widehat{\mathbb{A}}_{\infty}^{r}$ (vía the exponential isomorphism) and we use the Abel morphism (see [3]):

$$
\widehat{X}_{V} \stackrel{\phi_{1}}{\rightarrow} \mathcal{J}\left(\widehat{X}_{V}\right),
$$

which sends $T_{j}$ to the point in $\mathcal{J}\left(\widehat{X}_{V}\right)$ with coordinates:

$$
\left[T_{j}\right]:=\left((0, \ldots), \ldots,\left(T_{j}, \frac{T_{j}^{2}}{2}, \frac{T_{j}^{3}}{3}, \ldots\right), \ldots,(0, \ldots)\right) .
$$

It is clear that we have an addition map:

$$
\begin{aligned}
\widehat{X}_{V} \times \mathcal{J}\left(\widehat{X}_{V}\right) & \rightarrow \mathcal{J}\left(\widehat{X}_{V}\right) \\
\left(T_{\bullet}, t_{\bullet}\right) & \mapsto t_{\bullet}+\left[T_{\bullet}\right]
\end{aligned}
$$

where $T_{\bullet}=\left(T_{1}, \ldots, T_{r}\right), t_{\bullet}=\left(t^{(1)}, \ldots, t^{(r)}\right)$ and $t_{\bullet}+\left[T_{\bullet}\right]$ denotes the point in $\mathcal{J}\left(\widehat{X}_{V}\right)$ with coordinates $\left(\ldots, t_{i}^{(j)}+\frac{T_{j}^{i}}{i}, \ldots\right)$.

Remark D.23. (Remark 7.6) If $\Omega \in \operatorname{Gr}(k((z)))$, its BA-function is ([3, 33]):

$$
\psi_{\Omega}(z, t)=\exp \left(-\sum_{j \geq 1} \frac{t_{j}}{z^{j}}\right) \cdot\left(\frac{\tau_{\Omega}(t+[z])}{\tau_{\Omega}(t)}\right) .
$$

Remark D.24. For the case of points in $\operatorname{Gr}(k((z)))$, the Tau-function of a point $\Omega \in \operatorname{Gr}(k((z)))$ generates the subspace $\Omega$ ([33]). Nevertheless, this is no longer true for a point $W \in \operatorname{Gr}\left(V_{p}\right)$. In order to solve this problem, the following definition is used in [31] and [26].

Let us recall the decomposition $V_{p} \simeq V_{1} \times \cdots \times V_{r}$.

Definition D.25. (Definition 7.8 The $u$-th BA-function of a point $W \in \operatorname{Gr}\left(V_{p}\right)$ is the function:

$\psi_{u, W}\left(T_{\bullet}, t_{\bullet}\right):=\left(\xi_{u 1} \exp \left(-\sum_{j \geq 1} \frac{t_{j}^{(1)}}{T_{1}^{j}}\right) \frac{\tau_{W_{u 1}(}\left(t_{\bullet}+\left[T_{1}\right]\right)}{\tau_{W}\left(t_{\bullet}\right)}, \ldots, \xi_{u r} \exp \left(-\sum_{j \geq 1} \frac{t_{j}^{(n)}}{T_{r}^{j}}\right) \frac{\tau_{W_{u r}}\left(t_{\bullet}+\left[T_{r}\right]\right)}{\tau_{W}\left(t_{\bullet}\right)}\right)$,

where:

- $1 \leq u \leq r$.

- $W_{u v}:=\left(1, \ldots, T_{u}, \ldots, T_{v}^{-1}, \ldots, 1\right) \cdot W$. 
- $t_{\bullet}+\left[T_{v}\right]:=\left(t^{(1)}, \ldots, t^{(v)}+\left[T_{v}\right], \ldots, t^{(n)}\right)$.

- $\xi_{u i}$ is -1 if $i>u$ and 1 if $i \leq u$.

Theorem D.7.1. ([31], Theorem 3.6). Let us take $W \in \operatorname{Gr}^{0}\left(V_{p}\right)$. We thus have:

$$
\psi_{u, W}\left(T_{\bullet}, t_{\bullet}\right)=\left(1, \ldots, T_{u}, \ldots, 1\right) \cdot \sum_{i>0}\left(\psi_{u, W}^{i, 1}\left(T_{1}\right), \ldots, \psi_{u, W}^{i, r}\left(T_{r}\right)\right) p_{u i, W}\left(t_{\bullet}\right),
$$

where:

$$
\left\{\left(\psi_{u, W}^{i, 1}\left(T_{1}\right), \ldots, \psi_{u, W}^{i, r}\left(T_{r}\right)\right) \mid i>0,1 \leq u \leq n\right\}
$$

is a basis for $W$ and $p_{u i, W}\left(t_{\bullet}\right)$ are functions in $t_{\bullet}$.

Remark D.26. Let us recall that the superindex 0 of $\operatorname{Gr}^{0}\left(V_{p}\right)$ tells us that we are taking the connected component of the Grassmannian on which the index function of $W$ takes the value 0 (see Definition 3.3 of [3]).

To study the $m$-connected component $(m>0), \mathrm{Gr}^{m}\left(V_{p}\right)$, minor technical details are needed. In this case we have the following result.

Theorem D.7.2. ([31, Theorem 3.7]). If $W \in \mathrm{Gr}^{m}\left(V_{p}\right)$ we have:

$$
\psi_{u, W}\left(T_{\bullet}, t_{\bullet}\right)=v_{m}^{-1}\left(1, \ldots, T_{u}, \ldots, 1\right) \cdot \sum_{i>0}\left(\psi_{u, W}^{(i, 1)}\left(T_{1}\right), \ldots, \psi_{u, W}^{(i, r)}\left(T_{r}\right)\right) p_{u i, W}\left(t_{\bullet}\right),
$$

where

$$
\left\{\left(\psi_{u, W}^{(i, 1)}\left(T_{1}\right), \ldots, \psi_{u, W}^{(i, r)}\left(T_{t}\right)\right) \mid i>0,1 \leq u \leq t\right\}
$$

is a basis for $W$ and $p_{u i, W}\left(t_{\bullet}\right)$ are functions in $t_{\bullet}$.

In particular, an element of $V_{p}$ lies in $W$ if and only if it can be expressed as a linear combination of:

$$
\psi_{1, W}\left(T_{\bullet}, t_{\bullet}\right), \ldots, \psi_{r, W}\left(T_{\bullet}, t_{\bullet}\right)
$$

for certain values of the parameters $t_{\bullet}$.

Remark D.27. If $\Omega \in \operatorname{Gr}^{s}(k((z)))$, then ([33] $)$ :

$$
\psi_{\Omega}(z, t)=z^{1-s} \sum_{i>0} \psi_{\Omega}^{(i)}(z) p_{i}(t),
$$

where $\left\{\psi_{\Omega}^{(i)}(z)\right\}_{i}$ is a basis for $\Omega$ and $p_{i}(t)$ are functions in $t$. In particular, if $\Omega$ is the point defined by the canonical line bundle $\omega_{X}$, then $s=g-1$, where $g$ is the genus of $X$. If we denote $\Omega^{-1}$ as the point defined by $\omega_{X}^{-1}$, then $s=1-3 g$.

Since $V_{p}$ is a finite separable $k((z))$-algebra, it carries the metric of the trace:

$$
\operatorname{Tr}: V_{p} \times V_{p} \rightarrow k((z)),
$$

which is non-degenerate. Therefore, $V_{p}$ can be endowed with the non-degenerate pairing:

$$
\begin{aligned}
\mathrm{T}_{2}: V_{p} \times V_{p} & \rightarrow k \\
(a, b) & \mapsto \operatorname{Res}_{z=0}(\operatorname{Tr}(a, b)) d z .
\end{aligned}
$$


The pairing $\mathrm{T}_{2}$ induces an isomorphism of $k$-schemes:

$$
\begin{aligned}
R: \operatorname{Gr}\left(V_{p}\right) & \rightarrow \operatorname{Gr}\left(V_{p}\right) \\
W & \mapsto W^{\perp},
\end{aligned}
$$

where $W^{\perp}$ is written for the orthogonal of $W$ w.r.t. $\mathrm{T}_{2}$ (see [31, Lemma 3.8])

Definition D.28. The $u$-th Baker-Akhiezer function of a point $W \in \operatorname{Gr}\left(V_{p}\right)$ is:

$$
\begin{gathered}
\psi_{u, W}^{*}\left(T_{\bullet}, t_{\bullet}\right):=\psi_{u, W^{\perp}}\left(T_{\bullet},-t_{\bullet}\right) \\
\psi_{u, W}^{*}\left(T_{\bullet}, t_{\bullet}\right):= \\
=\left(\xi_{u 1} \exp \left(\sum_{j \geq 1} \frac{t_{j}^{(1)}}{T_{1}^{j}}\right) \frac{\tau_{W_{u 1}^{\perp}}\left(-t_{\bullet}+\left[T_{1}\right]\right)}{\tau_{W}^{\perp}\left(-t_{\bullet}\right)}, \ldots, \xi_{u n} \exp \left(\sum_{j \geq 1} \frac{t_{j}^{(n)}}{T_{n}^{j}}\right) \frac{\tau_{W_{u r}^{\perp}}\left(-t_{\bullet}+\left[T_{r}\right]\right)}{\tau_{W}^{\perp}\left(-t_{\bullet}\right)}\right)= \\
=\left(\xi_{u 1} \exp \left(\sum_{j \geq 1} \frac{t_{j}^{(1)}}{T_{1}^{j}}\right) \frac{\tau_{W_{1 u}}\left(t_{\bullet}-\left[T_{1}\right]\right)}{\tau_{W}\left(t_{\bullet}\right)}, \ldots, \xi_{u r} \exp \left(\sum_{j \geq 1} \frac{t_{j}^{(n)}}{T_{r}^{j}}\right) \frac{\tau_{W_{r u}}\left(t_{\bullet}-\left[T_{r}\right]\right)}{\tau_{W}\left(t_{\bullet}\right)}\right) .
\end{gathered}
$$

Theorem D.7.3. Let us take $W \in \mathrm{Gr}^{m}\left(V_{p}\right)$. We have:

$$
\psi_{u, W}^{*}\left(T_{\bullet}, t_{\bullet}\right)=v_{r-n-m}^{-1}\left(1, \ldots, T_{u}, \ldots, 1\right) \cdot \sum_{i>0}\left(\psi_{u, W}^{*(i, 1)}\left(T_{1}\right), \ldots, \psi_{u, W}^{*(i, r)}\left(T_{r}\right)\right) p_{u i, W}^{*}\left(t_{\bullet}\right),
$$

where:

$$
\left\{\left(\psi_{u, W}^{*(i, 1)}\left(T_{1}\right), \ldots, \psi_{u, W}^{*(i, r)}\left(T_{r}\right)\right) \mid i>0,1 \leq u \leq r\right\}
$$

is a basis for $W^{\perp}$ and $p_{u i, W}^{*}\left(t_{\bullet}\right)$ are functions in $t_{\bullet}$.

In particular, an element of $V_{p}$ lies in $W^{\perp}$ if and only if it can be expressed as a linear combination of:

$$
\psi_{1, W}^{*}\left(T_{\bullet}, t_{\bullet}\right), \ldots, \psi_{r, W}^{*}\left(T_{\bullet}, t_{\bullet}\right)
$$

for certain values of the parameters $t_{\bullet}$.

Let us define now the product of two BA-functions. Let us take $\Omega \in \operatorname{Gr}(k((z)))$ and $W \in \operatorname{Gr}\left(V_{p}\right)$. Let us consider its BA-functions $\psi_{\Omega}(z, t)$ and $\psi_{W}\left(T_{\bullet}, t_{\bullet}\right)$ respectively.

The graph, $\widehat{X}_{V} \rightarrow \widehat{X}_{V} \times \widehat{X}$, of the morphism $\widehat{\pi}: \widehat{X}_{V} \rightarrow \widehat{X}$ induces a map:

$$
f: \widehat{X}_{V} \times \Gamma_{V_{p}}^{-} \times \Gamma^{-} \rightarrow\left(\widehat{X}_{V} \times \Gamma_{V_{p}}^{-}\right) \times\left(\widehat{X} \times \Gamma^{-}\right) .
$$

Definition D.29. The product of the BA-functions of $\Omega \in \operatorname{Gr}(k((z)))$ and $W \in \operatorname{Gr}\left(V_{p}\right)$ is defined by:

$$
\psi_{W}\left(T_{\bullet}, t_{\bullet}\right) * \psi_{\Omega}(z, t):=f^{*}\left(\psi_{W}\left(T_{\bullet}, t_{\bullet}\right) \otimes_{k} \psi_{\Omega}(z, t)\right)
$$

The product of $\psi_{\Omega}(z, t)$ by the $u$-th BA-function of $W, \psi_{u, W}\left(T_{\bullet}, t_{\bullet}\right)$, can be defined in similar way. 
Proposition D.30. Let $W \in \operatorname{Gr}\left(V_{p}\right)$ and let $\Omega \in \operatorname{Gr}(k((z)))$ be such that $W \cdot \Omega \in \operatorname{Gr}\left(V_{p}\right)$. We thus have that:

$$
\left\{\psi_{\Omega}(z, t) * \psi_{1, W}\left(T_{\bullet}, t_{\bullet}\right), \ldots, \psi_{\Omega}(z, t) * \psi_{r, W}\left(T_{\bullet}, t_{\bullet}\right)\right\}
$$

is a generating system for $\Omega \cdot W$.

Recall that Theorem 4.15 says that a rational point $(W, p(T))$ of $\mathcal{U}_{X}^{\infty} \times \mathbb{A}$ lies on the image of:

$$
\mathcal{H i g g s}_{X}^{\infty} \hookrightarrow \mathcal{U}_{X}^{\infty} \times \mathbb{A}
$$

if and only if $T(W) \subseteq W \cdot \Omega$, where $\Omega \in \operatorname{Gr}(k((z)))$ is the point defined by the canonical line bundle $\omega_{X}$, and $T$ is the multiplication by $T$ in $V_{p}$. Bearing in mind the decomposition $V_{p} \simeq V_{1} \times \cdots \times V_{r}$, the image of $T$ is $T_{\bullet}=\left(T_{1}, \ldots, T_{r}\right)$. Therefore, if we think of $T$ as an operator in $V_{p}$ it is easy to see that $T$ is self-adjoint w.r.t. the pairing $\mathrm{T}_{2}$, that is:

$$
\mathrm{T}_{2}\left(T \cdot v, v^{\prime}\right)=\mathrm{T}_{2}\left(v, T \cdot v^{\prime}\right) \quad \forall v, v^{\prime} \in V_{p} .
$$

So that we get the equations describing $\mathcal{H}_{\text {iggs }}^{\infty}{ }_{X}^{\infty}$ :

Theorem D.7.4. (Theorem 7.20 Let $(W, p(T)) \in \mathcal{U}_{X}^{\infty} \times \mathbb{A}$ be a rational point, $\Omega \in \mathrm{Gr}^{g-1}(k((z)))$, and let us assume that $W \in \mathrm{Gr}^{m}\left(V_{p}\right)$ (with $m \neq \frac{1}{2}(r-n)$ ).

$(W, p(T))$ lies in the image of $\mathcal{H}$ iggs $s_{X}^{\infty} \hookrightarrow \mathcal{U}_{X}^{\infty} \times \mathbb{A}$ if and only if:

$$
\mathrm{T}_{2}\left(\frac{v_{r-n-m} \cdot T_{\bullet} \cdot \psi_{u, W}^{*}\left(T_{\bullet}, t_{\bullet}\right)}{\left(1, \ldots, T_{u}, \ldots, 1\right)}, \frac{v_{m} \cdot \psi_{\Omega^{-1}}(z, t) \cdot \psi_{v, W}\left(T_{\bullet}, t_{\bullet}\right)}{z^{3 g} \cdot\left(1, \ldots, T_{v}, \ldots 1\right)}\right)=0
$$

for all $1 \leq u, v \leq r$.

In order to compute explicit equations for the subscheme $\mathcal{H i g g s}_{X}^{\infty}(n)$ of $\mathcal{H i g g s}_{X}^{\infty}$ on which the spectral cover $\pi: X_{\varphi} \rightarrow X$ is totally ramified at $x\left(\pi^{-1}(x)=n y\right)$, BA-functions will be thought of as mere functions on $V_{p}$; that is, as polynomials on $T$ with coefficients in $k((z))$. In this way, we set:

$$
\begin{aligned}
& \Psi_{W}(z, T)=\sum_{i=0}^{n-1} \Psi_{W, i}(z) T^{i}, \quad \Psi_{W, i}(z) \in k((z)) \\
& \Psi_{W}^{*}(z, T)=\sum_{i=0}^{n-1} \Psi_{W, i}^{*}(z) T^{i}, \quad \Psi_{W, i}^{*}(z) \in k((z)) .
\end{aligned}
$$

Let $(E, \varphi, \phi)$ be a rational point of $\mathcal{H i g g s}_{X}^{\infty}$ and let:

$$
p_{\widehat{\varphi}}(T)=T^{n}-a_{1} T^{n-1}+\cdots+(-1)^{n} a_{n} \in \mathbb{A}
$$


be its formal characteristic polynomial (which now is irreducible). Newton-Girard identities (see Section I.2 of [25]) express $\operatorname{Tr}\left(T^{j}\right)$ in terms of $a_{i}=\operatorname{Tr}\left(\Lambda^{i} T\right)$ as follows:

$$
\operatorname{Tr}\left(T^{j}\right)=\left|\begin{array}{cccccc}
a_{1} & 1 & 0 & \cdot & \cdot & \cdot \\
2 a_{2} & a_{1} & 1 & 0 & \cdot & \cdot \\
3 a_{3} & a_{2} & a_{1} & 1 & 0 & \cdot \\
\cdot & \cdot & \cdot & \cdot & \cdot & \cdot \\
\cdot & \cdot & & & a_{1} & 1 \\
k a_{j} & a_{j-1} & a_{j-2} & \cdot & \cdot & a_{1}
\end{array}\right| \in k((z)), \quad \forall j \geq 0
$$

where $a_{j}=0$ for $j>n$.

Let us now consider the the subscheme $\operatorname{Higgs}_{X}^{\infty}(n)$ of $\mathcal{H i g g s} s_{X}^{\infty}$ for which the spectral cover $\pi: X_{\varphi} \rightarrow X$ is totally ramified at $x\left(\pi^{-1}(x)=n y\right)$. The equations are given by the following:

Theorem D.7.5. (Theorem 7.21) Let $(W, p(T))$ be a rational point of $\mathcal{U}_{X}^{\infty} \times \mathbb{A}$. Therefore, $(W, p(T))$ lies on the image of $\mathcal{H i g g s}_{X}^{\infty}(n) \hookrightarrow \mathcal{U}_{X}^{\infty} \times \mathbb{A}$ if and only if $p(T)$ is irreducible and:

$$
\sum_{k=0}^{2 n-2} \sum_{i+j=k} \operatorname{Res}_{z=0}\left(\Psi_{W, i}^{*}(z) \Psi_{\Omega^{-1}}(z) \Psi_{W, j}(z) \operatorname{Tr}\left(T^{k-1}\right)\right) \frac{d z}{z^{3 g}}=0,
$$

where $\operatorname{Tr}\left(T^{k-1}\right)$ can be expressed in terms of the coefficients of $p(T)$ by the equation (D.7.2).

\section{D.8. Conclusions.}

The aims of this work have been, firstly, to study in depth Hitchin's question mentioned in the introduction, complementing the results of [23, 12], and to look for another integrable system that behaves analogously as the Hitchin system does; secondly, to describe group schemes that locally generate moduli spaces. This second aim has interest not only on it's own nature, but also because it can be framed into the Bosonic String Theory (as mentioned in [34]), can be useful to compute the cohomology of standard moduli spaces (that is, without formal trivialization data), as noted in [4], and also allows us to think of the moduli spaces as quotient stacks of these groups, what can be seized, for example, to prove certain "strange dualities" of the Physics.

The general tactic we have chosen to proceed, consists of making use of the scheme structure of the Sato Grassmannian to embed on it (thanks to the Krichever morphism) moduli spaces, and to endow them with a scheme structure. One of the main advantages of the Krichever morphism is, that it allows to codify complicated moduli spaces into certainly easy algebraic conditions, that characterize them inside a Sato Grassmannian and show the existence of fine moduli spaces (as the moduli space of vector bundles) without imposing the stability conditions of the Geometric Invariant Theory of Mumford. Moreover, this is as 
useful as effective for the two applications we have chosen to work with: to compute tangent spaces and to give explicit KP-type equations.

We have obtained the following results:

1. To give a Krichever morphism for the moduli $\mathcal{U}^{\infty}$ of curves and vector bundles with formal trivialization data (without admitting local monodromy around the marked point and complementing the results of Mulase, [28]) and endow this moduli space with a scheme structure. We obtain a Mulase-like characterization of the image of the Krichever map only in terms of the point in the infinite Grassmannian defined by the vector bundle.

2. To compute the tangent space of $\mathcal{U}^{\infty}$ and to find out a group scheme that locally generates the moduli. This new group scheme is the group of semilinear automorphisms of a field of Laurent series, and its Lie algebra is an algebra of differential operators. We generalize in this fashion some of the results given in [4] and [32], where the case of line bundles is treated.

3. To characterize the image of the Krichever map for the moduli space $\mathcal{H}$ iggs $s_{X}^{\infty}$ of Higgs pairs over a smooth curve $X$ with formal trivialization data and to present this moduli as a subscheme of an infinite Grassmannian. The infinite Grassmannian we have needed to define the Krichever map is a fibration of infinite Grassmannians, $\mathcal{G}$ r, over the affine space $\mathbb{A}$ whose points are thought of as monic polynomials with coefficients in $k[[z]]$. This affine space $\mathbb{A}$ is nothing but a formal analogue of the so called Hitchin base. We prove that $\mathcal{H i g g s}_{X}^{\infty}$ is representable, that the Krichever map is injective and we also characterize its image. This allows us to prove moreover that $\mathcal{H i g g s}_{X}^{\infty}$ is representable by a closed subscheme of $\mathcal{U}_{X}^{\infty} \times \mathbb{A}$.

4. To characterize the image of the Krichever map for the moduli $\mathcal{C}_{0} v^{\infty}$ of finite coverings between pointed curves and the moduli $\mathcal{P} i c^{\infty}$ of line bundles over that coverings (always with formal trivialization data). The main difference between these moduli spaces and the ones treated in [32, 31] lies on the fact that we do not impose any compatibility among the formal trivializations, so that, we can recover those cases as particular ones. Using similar techniques to that of [32, 31], these moduli spaces can be endowed with a scheme structure inside the relative Grassmannian $\mathcal{G}$ r.

5. To find out an integrable system with similar behavior to that of Hitchin. If we fix the base curve $X$ and restrict ourselves to the subset $\mathbb{O} \subset \mathbb{A}$ of algebraic functions, the construction of $\mathcal{P} i c^{\infty}$ implies the existence of a scheme $\mathcal{P} i c_{X}^{\infty} \rightarrow \mathbb{O}$ parametrizing Picard schemes of all finite algebraic coverings of $X$, that is to say, the existence of an integrable system with analogue properties to those of the Hitchin system. Moreover, it turns out that $\mathcal{P} i c_{X}^{\infty}$ is a subscheme of $\mathcal{U}_{X}^{\infty}$ and one has a surjection $\mathcal{P} i c_{X}^{\infty} \rightarrow \mathcal{U}_{X}$ (where we write $\mathcal{U}_{X}$ to denote the stack of vector bundles over $X$ ), thus, we find here a relationship with the Hitchin Abelianization Program. It is worth to mention that $\mathcal{H i g g s}_{X}^{\infty}$ is a subscheme of $\mathcal{P} i c_{X}^{\infty}$. We expect that these results will allow us to provide a new construction of the spectral curves in the sense of [7]. 
6. To compute tangent spaces to $\operatorname{Cov}^{\infty}$ y $\mathcal{P} i c^{\infty}$ and to find out group schemes that locally generate them. These group schemes are, in the first case, a generalization of the Virasoro group studied in [34, 31] (it is a group of automorphisms of a field of Laurent series), and in the second one, a group of semilinear automorphisms whose Lie algebra is an algebra of differential operators, which can be obtained as an extension of the Heisenberg algebra studied in [1].

7. To compute hierarchies of differential equations describing the moduli space of Higgs pairs, $\mathcal{H i g g s} s_{X}^{\infty}$. This hierarchies are equivalent to the algebraic equations of $\mathcal{H}$ iggs $s_{X}^{\infty}$ as a subscheme of the Grassmannian. For this purpose, the key ingredients are, on one side the characterization of the image of the Krichever map (explained in 3), and, on the other side, the fact that we have been able to reformulate in our context the formal geometry described in [3] and [31], what have allowed us to give the equations in terms of Baker-Akhiezer functions. This also offers a complementary aproach to that of [23] (non ramified spectral cover) to explicitely compute the equations. Moreover, for the subscheme of $\mathcal{H}_{\text {iggs }}^{\infty}{ }_{X}^{\infty}$ on which the spectral cover is totally ramified at the marked point, we give the equations just in terms of the characteristic coefficients of the Higgs field. We also hope that these techniques allow us to study the very recent and interesting results of Mulase and Hodge, contained in [29], which relates the theory of KP-type equations with Mirror Symmetry and Langlands duality. 


\section{Índice alfabético}

Baker-Akhiezer, véase función

coeficientes característicos, 50

conmensurable, 127, 128, 139

curva

espectral, 50

espectral formal, 60

derivaciones equivariantes, 97, 103

discreto

subespacio, 129

submódulo, 132

espacio tangente

curvas, $83-85$

curvas y fibrados, 90, 91, 95

fibrados, 87,89

Grassmanniana, 85, 136

Grassmanniana relativa, 103

haces de línea en revestimientos, 104, 105 .

107

pares de Higgs, 108,109

revestimientos, 98, 100, 103

estabilizador, 40, 71,72

fórmula de Newton-Girard, 14, 122

función

de Baker-Akhiezer, 115

adjunta, 118

producto de, 119

Tau, 114

Grassmanniana infinita, 37, 129, 132

relativa, 61, 68

grupo de automorfismos, 11, 12, 84, 85, 101

semilineales, 19, 21, 93, 106

de Heisenberg, 17, 73, 79

de invertibles, 9, 17, 112

de Weyl, 17

simétrico,11, 16

Heisenberg, véase grupo

hipercohomología, 83

Jacobiana formal, 112, 115

Krichever, véase morfismo

métrica de la traza, 117

Moduli de

curvas, 35

curvas y fibrados, 36

haces de línea en revestimientos, 70

pares de Higgs, 51

revestimientos, 66

morfismo

de Abel,113, 115

de Hitchin, 50

de Hitchin formal, 55, 60, 73

de Krichever

construcción inversa, 139

curvas y fibrados, $37, \sqrt{39}$

espectral, 62

haces de línea en revestimientos, 71

pares de Higgs, 55

revestimientos, 67, 68

símbolo, 28, 146

traza, 13,69

Newton-Girard, véase fórmula de 
operadores diferenciales, 28, 30, 31, 144

con símbolo escalar, 28, 30,146

par de Higgs, 49

símbolo, véase morfismo

sistema integrable, 74,76

Tau, véase función

topología étale, 13, 102

traza, véase morfismo

Weyl, véase grupo 


\section{Bibliografía}

[1] M. R. AdAmS AND M. J. BeRgVelt, The Krichever map, vector bundles over algebraic curves, and Heisenberg algebras, Comm. Math. Phys., 154 (1993), pp. 265-305.

[2] A. Álvarez VÁzQueZ, Estructuras aritméticas de las curvas algebraicas. PhD Thesis, 1996.

[3] A. Alvarez Vázquez, J. M. Muñoz Porras, and F. J. Plaza Martín, The algebraic formalism of soliton equations over arbitrary base fields, in Workshop on Abelian Varieties and Theta Functions (Spanish) (Morelia, 1996), vol. 13 of Aportaciones Mat. Investig., Soc. Mat. Mexicana, México, 1998, pp. 3-40.

[4] E. Arbarello, C. De Concini, V. G. Kac, And C. Procesi, Moduli spaces of curves and representation theory, Comm. Math. Phys., 117 (1988), pp. 1-36.

[5] M. F. AtiYAh AND I. G. MAcDonald, Introduction to commutative algebra, Addison-Wesley Publishing Co., Reading, Mass.-London-Don Mills, Ont., 1969.

[6] A. Beauville And Y. Laszlo, Conformal blocks and generalized theta functions, Comm. Math. Phys., 164 (1994), pp. 385-419.

[7] A. Beauville, M. S. Narasimhan, And S. Ramanan, Spectral curves and the generalised theta divisor, J. Reine Angew. Math., 398 (1989), pp. 169-179.

[8] D. Ben-Zvi And E. Frenkel, Spectral curves, opers and integrable systems, Publ. Math. Inst. Hautes Études Sci., (2001), pp. 87-159.

[9] F. BotTacin, Symplectic geometry on moduli spaces of stable pairs, Ann. Sci. École Norm. Sup. (4), 28 (1995), pp. 391-433.

[10] K. S. Brown, Cohomology of groups, vol. 87 of Graduate Texts in Mathematics, Springer-Verlag, New York, 1994. Corrected reprint of the 1982 original.

[11] J. Burchnall And T. Chaundy, Commutative ordinary differential operators, in Proceedings of the London Mathematical Society, 1922, pp. 420-440. 
[12] R. DonAgi AND E. MARKMAN, Spectral covers, algebraically completely integrable, Hamiltonian systems, and moduli of bundles, in Integrable systems and quantum groups (Montecatini Terme, 1993), vol. 1620 of Lecture Notes in Math., Springer, Berlin, 1996, pp. 1-119.

[13] E. FRENKEL, Langlands correspondence for loop groups, vol. 103 of Cambridge Studies in Advanced Mathematics, Cambridge University Press, Cambridge, 2007.

[14] _ Lectures on the Langlands program and conformal field theory, in Frontiers in number theory, physics, and geometry. II, Springer, Berlin, 2007, pp. 387-533.

[15] E. Frenkel And D. Ben-Zvi, Vertex algebras and algebraic curves, vol. 88 of Mathematical Surveys and Monographs, American Mathematical Society, Providence, RI, second ed., 2004.

[16] P. Griffiths AND J. HARRIS, Principles of algebraic geometry, Wiley Classics Library, John Wiley \& Sons Inc., New York, 1994. Reprint of the 1978 original.

[17] A. GROTHENDIECK, Éléments de géométrie algébrique. IV. Étude locale des schémas et des morphismes de schémas IV, Inst. Hautes Études Sci. Publ. Math., (1967), p. 361.

[18] J. Harris AND I. MorRison, Moduli of curves, vol. 187 of Graduate Texts in Mathematics, Springer-Verlag, New York, 1998.

[19] R. Hartshorne, Algebraic geometry, Springer-Verlag, New York, 1977. Graduate Texts in Mathematics, No. 52.

[20] _ Lectures on deformation theory. 2004.

[21] N. Hitchin, Stable bundles and integrable systems, Duke Math. J., 54 (1987), pp. $91-$ 114.

[22] I. KRICHEVER, Methods of algebraic geometry in the theory of nonlinear equations, Russ. Math.Surv., 32 (1977), pp. 185-214.

[23] Y. Li and M. Mulase, Hitchin systems and KP equations, Internat. J. Math., 7 (1996), pp. 227-244.

[24] _ Prym varieties and integrable systems, Comm. Anal. Geom., 5 (1997), pp. 279332.

[25] I. G. Macdonald, Symmetric functions and Hall polynomials, The Clarendon Press Oxford University Press, New York, 1979. Oxford Mathematical Monographs.

[26] A. MAFFEI, The multicomponent KP and Fay trisecant formula, Internat. Math. Res. Notices, (1996), pp. 769-791. 
[27] M. Mulase, Cohomological structure in soliton equations and Jacobian varieties, J. Differential Geom., 19 (1984), pp. 403-430.

[28] _ Category of vector bundles on algebraic curves and infinite-dimensional Grassmannians, Internat. J. Math., 1 (1990), pp. 293-342.

[29] M. Mulase And A. Hodge, Hitchin integrable systems, deformations of spectral curves and kp-type equations. Preprint math.AG/0801.0015.v1.

[30] D. MUMFord, An algebro-geometric construction of commuting operators and of solutions to the Toda lattice equation, Korteweg deVries equation and related nonlinear equation, in Proceedings of the International Symposium on Algebraic Geometry (Kyoto Univ., Kyoto, 1977), Tokyo, 1978, Kinokuniya Book Store, pp. 115-153.

[31] J. M. Muñoz Porras And F. J. Plaza Martín, Equations of hurwitz schemes in the infinite grassmannian. Preprint arXiv:math.AG/0207091v3. To appear in Math. Nachr.

[32] _ _ Virasoro groups and hurwitz schemes. Preprint math.AG/0502023.

[33] _ Equations of the moduli of pointed curves in the infinite Grassmannian, J. Differential Geom., 51 (1999), pp. 431-469.

[34] - Automorphism group of $k((t))$ : applications to the bosonic string, Comm. Math. Phys., 216 (2001), pp. 609-634.

[35] F. Plaza Martín, Estudio algebraico de la ecuaciones kp: Ecuaciones de las variedades de moduli de curvas y de variedades de prym. PhD Thesis, 1997.

[36] _ Relative infinite grassmannian. Work in progress, 2008.

[37] F. J. Plaza Martín, Prym varieties and the infinite Grassmannian, Internat. J. Math., 9 (1998), pp. 75-93.

[38] A. Pressley and G. Segal, Loop groups, Oxford Mathematical Monographs, The Clarendon Press Oxford University Press, New York, 1986. , Oxford Science Publications.

[39] P. SAMUEL, Lectures on old and new results on algebraic curves, Notes by S. Anantharaman. Tata Institute of Fundamental Research Lectures on Mathematics, No. 36, Tata Institute of Fundamental Research, Bombay, 1966.

[40] M. SATo AND Y. SATo, Soliton equations as dynamical systems on infinitedimensional Grassmann manifold, in Nonlinear partial differential equations in applied science (Tokyo, 1982), vol. 81 of North-Holland Math. Stud., North-Holland, Amsterdam, 1983, pp. 259-271.

[41] G. SEGAL, The definition of conformal field theory. Unpublished manuscript, 1990. 
[42] G. Segal And G. Wilson, Loop groups and equations of KdV type, Inst. Hautes Études Sci. Publ. Math., (1985), pp. 5-65.

[43] C. S. SESHADRI, Fibrés vectoriels sur les courbes algébriques, vol. 96 of Astérisque, Société Mathématique de France, Paris, 1982. Notes written by J.-M. Drezet from a course at the École Normale Supérieure, June 1980.

[44] T. SHIOTA, Characterization of Jacobian varieties in terms of soliton equations, Invent. Math., 83 (1986), pp. 333-382.

[45] C. T. Simpson, Moduli of representations of the fundamental group of a smooth projective variety. II, Inst. Hautes Études Sci. Publ. Math., (1994), pp. 5-79 (1995).

[46] K. UENO, Introduction to conformal field theory with gauge symmetries, in Geometry and physics (Aarhus, 1995), vol. 184 of Lecture Notes in Pure and Appl. Math., Dekker, New York, 1997, pp. 603-745.

[47] J. WeHLer, Cyclic coverings: deformation and Torelli theorem, Math. Ann., 274 (1986), pp. 443-472.

[48] G. E. Welters, Polarized abelian varieties and the heat equations, Compositio Math., 49 (1983), pp. 173-194. 